SLAC-R-959

\title{
RADIO FREQUENCY STATION - BEAM DYNAMICS INTERACTION IN CIRCULAR ACCELERATORS
}

\author{
A DISSERTATION \\ SUBMITTED TO THE DEPARTMENT OF ELECTRICAL \\ ENGINEERING \\ AND THE COMMITTEE ON GRADUATE STUDIES \\ OF STANFORD UNIVERSITY \\ IN PARTIAL FULFILLMENT OF THE REQUIREMENTS \\ FOR THE DEGREE OF \\ DOCTOR OF PHILOSOPHY
}

Themistoklis Mastoridis

August 2010

Work supported in part by US Department of Energy contract DE-AC02-76SF00515. 
(C) 2005 by Themistoklis Mastoridis. All Rights Reserved.

Re-distributed by Stanford University under license with the author.

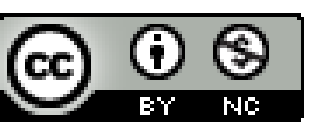

This work is licensed under a Creative Commons AttributionNoncommercial 3.0 United States License.

http://creativecommons.org/licenses/by-nc/3.0/us/

This dissertation is online at: http://purl.stanford.edu/nz794nw1943 
I certify that I have read this dissertation and that, in my opinion, it is fully adequate in scope and quality as a dissertation for the degree of Doctor of Philosophy.

Sami Tantawi, Primary Adviser

I certify that I have read this dissertation and that, in my opinion, it is fully adequate in scope and quality as a dissertation for the degree of Doctor of Philosophy.

John Fox

I certify that I have read this dissertation and that, in my opinion, it is fully adequate in scope and quality as a dissertation for the degree of Doctor of Philosophy.

Ivan Linscott

I certify that I have read this dissertation and that, in my opinion, it is fully adequate in scope and quality as a dissertation for the degree of Doctor of Philosophy.

Ronald Ruth

Approved for the Stanford University Committee on Graduate Studies.

Patricia J. Gumport, Vice Provost Graduate Education

This signature page was generated electronically upon submission of this dissertation in electronic format. An original signed hard copy of the signature page is on file in University Archives. 


\section{Abstract}

The longitudinal beam dynamics in circular accelerators is mainly defined by the interaction of the beam current with the accelerating Radio Frequency (RF) stations. For stable operation, Low Level RF (LLRF) feedback systems are employed to reduce coherent instabilities and regulate the accelerating voltage. The LLRF system design has implications for the dynamics and stability of the closed-loop RF systems as well as for the particle beam, and is very sensitive to the operating range of accelerator currents and energies. Stability of the RF loop and the beam are necessary conditions for reliable machine operation.

This dissertation describes theoretical formalisms and models that determine the longitudinal beam dynamics based on the LLRF implementation, time domain simulations that capture the dynamic behavior of the RF station-beam interaction, and measurements from the Positron-Electron Project (PEP-II) and the Large Hadron Collider (LHC) that validate the models and simulations. These models and simulations are structured to capture the technical characteristics of the system (noise contributions, non-linear elements, and more). As such, they provide useful results and insight for the development and design of future LLRF feedback systems. They also provide the opportunity to study diverse longitudinal beam dynamics effects such as coupled-bunch impedance driven instabilities and single bunch longitudinal emittance growth.

Coupled-bunch instabilities and RF station power were the performance limiting effects for PEP-II. The sensitivity of the instabilities to individual LLRF parameters, the effectiveness of alternative operational algorithms, and the possible tradeoffs between RF loop and beam stability were studied. New algorithms were implemented, 
with significant performance improvement leading to a world record current during the last PEP-II run of $3212 \mathrm{~mA}$ for the Low Energy Ring.

Longitudinal beam emittance growth due to RF noise is a major concern for LHC. Simulations studies and measurements were conducted that clearly show the correlation between RF noise and longitudinal bunch emittance, identify the major LLRF noise contributions, and determine the RF component dominating this effect. With these results, LHC upgrades and alternative algorithms are evaluated to reduce longitudinal emittance growth during operations.

The applications of this work are described with regard to future machines and analysis of new technical implementations, as well as to possible future work which would continue the directions of this dissertation. 


\section{Acknowledgments}

I would like to thank my advisers Prof. John D. Fox and Prof. Sami Tantawi. I am grateful to John for introducing me to accelerator physics and believing in me through the more twisted turns of this journey. John has always motivated me with his spherical knowledge of accelerator physics and his clear long term research vision. I am indebted to Prof. Sami Tantawi for his support and trust in me. Sami also instilled a mathematical clarity in this work.

I was very lucky to be surrounded by exceptional people at the Advanced Electronics group at the Stanford Linear Accelerator Center (SLAC). Dmitry Teytelman has always been an inspiration with his ability to quickly grasp the core of every problem and provide an enlightening point of view. Dan Van Winkle has helped me increase my understanding of hardware. I owe so much to Claudio Rivetta that is hard to express in this limited space. I hope he knows how appreciative I am for his patience all these years. Collaborating with him has been a very educational and rewarding experience.

I would like to thank Prof. Ron Ruth for sharing his accelerator physics wisdom during our conversations, in particular during the development of the LHC beam diffusion formalism. Dr. Ivan Linscott has always helped me stay on track and focus on the long term goals. His comments and ideas have been essential to the clarity and organization of this work.

I am grateful to the SLAC AARD department for hosting me all these years. SLAC and DOE's LHC American Research Program have supported my graduate studies.

A substantial part of this work would not have been possible without the help, 
interest, and hospitality of the CERN BE-RF group, and in particular Philippe Baudrenghien.

I have been fortunate to meet, learn from and be supported by Prof. Piero Pianetta, Prof. Alex Chao, Dr. Sam Heifets, Prof. Umran Inan, and many more people over these years.

My love and thanks to my friends for being there during good and bad times, es-

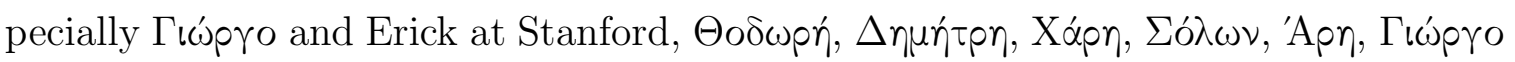
in Greece. Many thanks to the Paquin and Miller families.

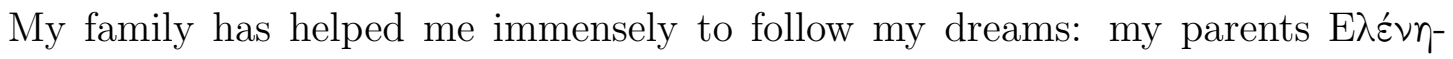

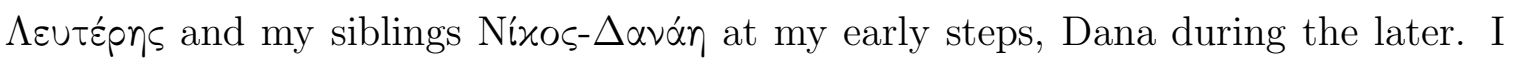
thank and love them all. This dissertation is dedicated to them. 


\section{Contents}

Abstract iv

Acknowledgments $\quad$ vi

1 Introduction 1

1.1 Beam Dynamics and the RF station . . . . . . . . . . . 2

1.2 RF Station-Beam Dynamics Interaction . . . . . . . . . . . . . 3

1.3 PEP-II Time-Domain Simulation: Motivation and Development . . . 3

1.4 PEP-II Longitudinal Coupled-Bunch Instabilities Studies . . . . . . 4

1.5 RF System Models for the LHC with Application to Longitudinal Dy-

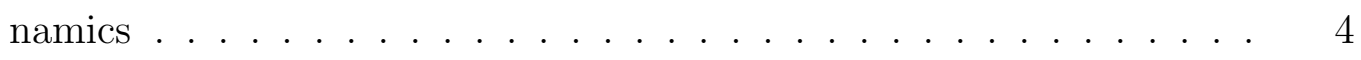

1.6 LHC Longitudinal Beam Dynamics Studies . . . . . . . . . . . . 4

1.7 LHC Longitudinal Beam Emittance Dependence on RF Noise: Mea-

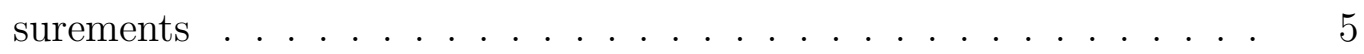

1.8 Summary $\ldots \ldots \ldots \ldots \ldots \ldots \ldots \ldots \ldots \ldots \ldots \ldots \ldots \ldots \ldots$

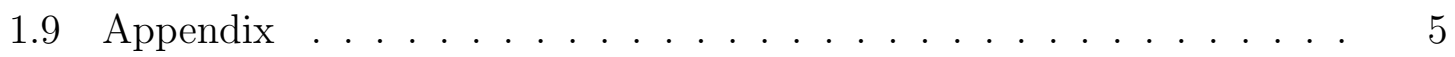

2 Beam Dynamics and the RF station $\quad 7$

2.1 Single-bunch Beam Dynamics . . . . . . . . . . . . 8

2.1.1 Equations of Motion . . . . . . . . . . . . 10

2.1 .2 Equilibrium Point Stability _. . . . . . . . . 11

2.2 Coupled-bunch Dynamics _. . . . . . . . . . . . . . . 13

2.2 .1 Equilibrium Point Stability $\ldots \ldots \ldots \ldots \ldots$

2.2.2 Third-Harmonic Cavity Example . . . . . . . . . . . 16 
2.2 .3 Modal Domain . . . . . . . . . . . . . . . . . . . 17

2.3 Introducing Impedances $\ldots \ldots \ldots$

2.4 Sources of Impedance . . . . . . . . . . . . . . . . . . . . 20

3 RF Station-Beam Dynamics Interaction $\quad 22$

3.1 Pedersen Model of the RF Station-Beam Interaction . . . . . . . . . 24

3.2 Feedback Control Systems . . . . . . . . . . . . . . . . 27

3.2 .1 Passive Cures . . . . . . . . . . . . . . . . . . . 28

3.2 .2 Active Feedback Control . . . . . . . . . . . . . . . . 29

3.2.3 Active Feedback Implementation Example . . . . . . . . . . . 31

4 PEP-II Time-Domain Simulation: Motivation and Development 33

4.1 The Simulation's Motivation and Goals . . . . . . . . . . . . . 34

4.2 PEP-II Rings - Longitudinal Systems . . . . . . . . . . . . . . 34

4.3 Model Description . . . . . . . . . . . . . . . 36

4.4 Identification and Configuration Algorithms . . . . . . . . . . 42

4.5 Transfer Function Validation in PEP-II . . . . . . . . . . . . . 46

4.6 Growth Rate Measurement Techniques . . . . . . . . . . . . . 48

4.7 Growth Rate Validation . . . . . . . . . . . . . . . . . 52

5 PEP-II Longitudinal Coupled-Bunch Instabilities Studies 55

5.1 Defining System Stability _. . . . . . . . . . . 56

5.1 .1 Operation Point Stability _ . . . . . . . . . . 56

5.1.2 Operation Point Robustness To Perturbations . . . . . . . . 57

5.2 Stability Criteria for PEP-II _ . . . . . . . . . . . . . . 59

5.2.1 Klystron Power Requirements, Impact of Klystron Characteristics 59

5.2.2 Beam Current Limits due to Low-mode Instability

Growth Rates . . . . . . . . . . . . . . . . 60

5.3 Beam Current Limits due to the RF System . . . . . . . . . . 61

$5.3 .1 \quad$ HER . . . . . . . . . . . . . . . . . . . . 62

$5.3 .2 \quad$ LER . . . . . . . . . . . . . . . . . . . . . 63

5.4 Growth Rate Sensitivity Analysis . . . . . . . . . . . . 65 
5.4.1 Growth Rate Dependence on Gap Voltage . . . . . . . . . . . 70

5.5 LLRF System Imperfections . . . . . . . . . . . . . . . . . . . 71

5.6 Beam Current Limits due to Growth Rates . . . . . . . . . . . . . . 75

5.6 .1 HER .......................... 75

5.6 .2 LER . . . . . . . . . . . . . . . . . 77

5.7 Technical Upgrades' Effect on Real Machine Performance . . . . . . . 82

5.8 Conclusions on PEP-II Simulation Studies . . . . . . . . . . . . . . 89

6 RF System Models for the LHC with Application to Longitudinal $\begin{array}{ll}\text { Dynamics } & 91\end{array}$

6.1 LHC Simulation Motivation . . . . . . . . . . . . . . . . . 92

6.1.1 Identification and Configuration Tools . . . . . . . . . . . 92

6.2 LHC, PEP-II LLRF Similarities . . . . . . . . . . . . . . . . . 93

6.2.1 System Description . . . . . . . . . . . . . . . 94

6.3 Simulation and Model Description . . . . . . . . . . . . . 96

6.4 Simulation Validation . . . . . . . . . . . . . . . . . . . . 98

7 LHC Longitudinal Beam Dynamics Studies 102

7.1 New Directions for LHC . . . . . . . . . . . . . . . . . . 103

7.2 Noise Sources . . . . . . . . . . . . . . . . . . . . . 104

7.3 Formalism for Bunch Length Estimation . . . . . . . . . . . . . 106

7.4 An Application to Single-bunch Dynamics . . . . . . . . . . . . 113

7.4.1 Transfer Function Estimation: Time-domain Simulation . . . . 114

7.4.2 RF Station Configurations of Interest . . . . . . . . . . . . . . 114

7.4.3 Noise Thresholds for Longitudinal Beam Diffusion . . . . . . . 117

7.5 Multi-bunch Stability Studies . . . . . . . . . . . . . . . . 122

7.5.1 Stability Criterion . . . . . . . . . . . . . . . 124

7.5.2 Growth Rate Sensitivity to LLRF Parameters . . . . . . . . . 126

8 RF Noise Effects on LHC Beam Diffusion 128

8.1 System Description . . . . . . . . . . . . . . . . . . 129

8.2 RF Noise Dominating Component . . . . . . . . . . . . . . . . 130 
8.3 Beam Diffusion Dependence on RF Noise . . . . . . . . . . . . . . . . 132

8.4 LLRF Feedback Noise . . . . . . . . . . . . . . . . . . . . . . 138

8.5 LLRF Feedback Noise Dependence on RF Feedback Gain Settings . . 141

9 Conclusions and Future Research Directions 143

9.1 Main Contributions . . . . . . . . . . . . . . . . . . 144

9.2 Future Research Directions . . . . . . . . . . . . . . . . . 145

A PEP-II HOM Driven Instabilities $\quad 148$

A.1 Longitudinal Instabilities in PEP-II . . . . . . . . . . . . . . . . 148

A.2 HOM-driven Modes: Growth and Damping Rates . . . . . . . . . . . 150

A.2.1 HER . . . . . . . . . . . . . . . . . . . 152

A.2.2 LER . . . . . . . . . . . . . . . . . . . 154

A.3 Feedback Model . . . . . . . . . . . . . . . . . . . 156

A.4 Noise Floor Measurements . . . . . . . . . . . . . . . . . . . . . . . 162

A.4.1 LER Noise Measurements . . . . . . . . . . . . . . . 163

A.4.2 HER Noise Measurements . . . . . . . . . . . . . . . . 164

A.5 Conclusions .......................... 165

B PEP-X Design Studies 167

B.1 Longitudinal Stability . . . . . . . . . . . . . . . 168

B.1.1 HOM-driven Coupled-bunch Longitudinal Instabilities . . . . . 168

B.1.2 Coupled-bunch Longitudinal Instabilities Driven by the Fundamental Mode and Third-harmonic System . . . . . . . . . . 174

B.2 RF Station Noise . . . . . . . . . . . . . . . . . 176

C PEP-II Driver Amplifier Distortion Data 179

C.0.1 Small Signal Transfer Function . . . . . . . . . . . . . 181

C.0.2 Intermodulation Plots . . . . . . . . . . . . . . . . 182

D Summary of Accelerator Parameters 186

$\begin{array}{lr}\text { Bibliography } & 188\end{array}$ 


\section{List of Tables}

5.1 LER operation point limits. . . . . . . . . . . . . . . . . 61

5.2 Growth Rate Sensitivity Table. . . . . . . . . . . . . . . 68

5.3 Growth Rate vs. Station Table. . . . . . . . . . . . . . . . . . . 74

5.4 LER limiting factors and maximum current for each configuration . . 86

7.1 RF and LLRF parameters for beginning/end of injection and physicscollision configurations considered in this Chapter. . . . . . . . . 116

7.2 Modulator and LLRF noise threshold in $\mathrm{pV} / \sqrt{\mathrm{Hz}}$ for injection configurations. . . . . . . . . . . . . . . . . . . . . . 119

7.3 Modulator and LLRF noise threshold in $\mu \mathrm{V} / \sqrt{\mathrm{Hz}}$ for physics configurations. . . . . . . . . . . . . . . . . . . . . . . 119

7.4 Growth Rates of the most unstable mode and maximum tune shifts for each configuration, with the 1-Turn Feedback on. . . . . . . . 125

7.5 Growth Rate Sensitivity on LLRF parameters. . . . . . . . . . . 127

8.1 Bunch Growth Rate Dependence on BPL gain and Noise Power for B1 135

8.2 Bunch Growth Rate Dependence on BPL gain and Noise Power for B2 137

8.3 Component wise contribution in $\mathrm{mV}$ rms. . . . . . . . . . . . 139

B.1 Cavity Resonances . . . . . . . . . . . . . . . . . . . . . . . . 169

D.1 PEP-II and LHC Beam parameters . . . . . . . . . . . 186

D.2 PEP-II and LHC RF parameters . . . . . . . . . . . 187 


\section{List of Figures}

2.1 RF cavity voltage as a function of time. . . . . . . . . . 8

2.2 Equilibrium point shift and voltage slope change with addition of Third-Harmonic cavity. . . . . . . . . . . . . . . . . . . 17

2.3 PEP-II estimated longitudinal HOM impedance per cavity, $\sum Z_{i}^{\|} . \quad$. 21

3.1 RF station-beam interaction through the cavity impedance. . . . . . 23

3.2 Representation of the system's pole with LLRF and damping loops (closed loop pole o), the pole with the LLRF feedback (open loop pole $\mathrm{x})$, and in the absence of any feedback $(\mathrm{red} *) \ldots \ldots \ldots . . \ldots 24$

3.3 Pedersen model block diagram. . . . . . . . . . . . . . . . . 26

3.4 Block diagram representation of the RF station-beam interaction. . . 26

3.5 Phasor Diagram at $\omega_{R F}$ of the RF station-beam interaction - heavy beam loading. . . . . . . . . . . . . . . . . . . . . 27

3.6 Phasor Diagram at $\omega_{R F}$ of the RF station-beam interaction - light beam loading. . . . . . . . . . . . . . . . . . . . . 27

3.7 PEP-II cavity with rectangular waveguide ports. . . . . . . . . 28

3.8 Installed PEP-II cavity with damping waveguides. . . . . . . . . 28

3.9 LLRF Feedback Loops. . . . . . . . . . . . . . . . . . . . . . . . . . . . 29

3.10 Naked cavity impedance. . . . . . . . . . . . . . . . . . . . 31

3.11 Naked cavity growth rates. . . . . . . . . . . . . . . . . 31

3.12 Impedance reduction due to the Direct Loop. . . . . . . . . . . . . 32

3.13 Growth rate reduction due to the Direct Loop. . . . . . . . . . . . . 32

3.14 Impedance reduction due to the Comb and Direct Loop. . . . . . . . 32

3.15 Growth rate reduction due to the Comb and Direct Loop. . . . . . . . 32 
4.1 PEP-II RF station block diagram. . . . . . . . . . . . . . 35

4.2 Simulink Block Diagram for PEP-II LER. . . . . . . . . . . . . . . . 37

4.3 Simplified System Block Diagram. . . . . . . . . . . . . . . . 38

4.4 Successive optimization of RF station transfer function. The first transfer function demonstrates peaking, which is reduced in two steps by appropriate adjustments of the LLRF gain and phase. . . . . . . .

4.5 Transfer Function and parameters of operating station in LER at 1400 mA. . . . . . . . . . . . . . . . . . 47

4.6 Transfer Function and parameters of macrostation from non-linear simulation in the LER at $1400 \mathrm{~mA}$. . . . . . . . . . . . . . . . . . . 48

4.7 Time-domain data showing the phase deviation for 20 bunches over 2.5 ms (about 340 turns). The amplitude of the synchrotron oscillation is growing for all the bunches. . . . . . . . . . . . . . . . . 50

4.8 Simulated Low Frequency Beam Modes (LER at $2500 \mathrm{~mA}$ ). . . . . . 51

4.9 Plot of $\Delta_{l}$ with current in $m A$. The $-6 \mathrm{~ms}^{-1}$ limit is from the lowmode longitudinal control path. . . . . . . . . . . . . 52

4.10 Measured and Simulated Growth Rates for the LER (Simulated for each station as well as the macroklystron). Most unstable mode is -3 .

5.1 Power Curves for SLAC \#5 klystron. For a desired output power the operation point is defined by the HVPS level and the input power. . .

5.2 HER maximum achievable beam current and total forward power as a

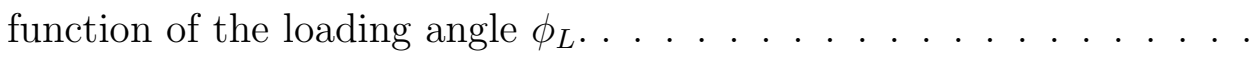

5.3 HER maximum achievable beam current versus gap voltage. The 18.5 MV operation point is marked with a circle. . . . . . . . . . . 64

5.4 LER Klystron Forward Power versus current. . . . . . . . . . . . . . 65

5.5 LER Klystron Forward Power versus Gap Voltage. . . . . . . . . . . . 66

5.6 Modes -10 to 10 for nominal case. . . . . . . . . . . . . . . 67

5.7 Direct Loop Phase. $+10^{\circ}$ rotation in green, $-10^{\circ}$ rotation in red, and the nominal case for reference in blue. . . . . . . . . . . . . . . . . . 
5.8 Comb Loop Phase. $+10^{\circ}$ rotation in green, $-10^{\circ}$ rotation in red, and the nominal case for reference in blue. . . . . . . . . . . . . . 70

5.9 Growth Rates with Direct and Comb loop phase rotation for the LER at $1400 \mathrm{~mA} \ldots \ldots \ldots 71$

5.10 Measured and simulated growth rates vs. comb phase rotation for the LER at $1400 \mathrm{~mA}$. Agreement in both the general form and most unstable mode number. . . . . . . . . . . . . . . . . .

5.11 Growth Rates for different number of station and gap voltage for the LER. . . . . . . . . . . . . . . . . . . . . . 73

5.12 Driver Amplifier Transfer Function driven by carrier (Large Signal Re-

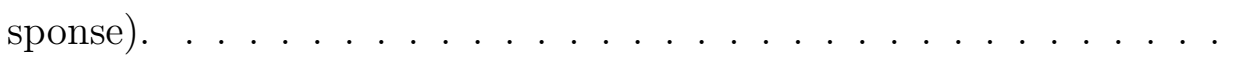

5.13 Driver Amplifier Transfer Function driven by carrier and modulation (Small-Signal Response). The modulation is swept across the band at a level $-30 \mathrm{~dB}$ below the power carrier at $476 \mathrm{MHz}$. . . . . . . . . . . 76

5.14 HER Growth Rates of the most unstable low-order beam mode (mode $-3) \ldots \ldots \ldots \ldots \ldots \ldots \ldots \ldots$

5.15 Estimated Growth Rates at $4.05 \mathrm{MV}$ for the configuration during run $5 \mathrm{~b}$ and for upgraded implementations including improved klystron driver amplifiers, different comb loop phase margins, and more SLAC klystrons. . . . . . . . . . . . . . . . . 78

5.16 Damping margin definition. . . . . . . . . . . . . . 80

5.17 Estimated damping margins at 4.05 MV for the configuration during run $5 \mathrm{~b}$ and for upgraded implementations including improved klystron driver amplifiers, different comb loop phase margins, and more SLAC

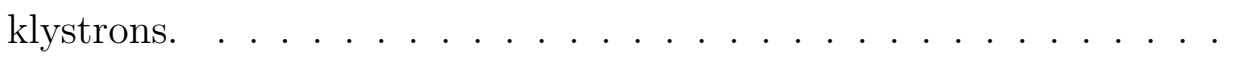

5.18 Estimated Growth Rates at 4.5 MV for the configuration during run 5b and for upgraded implementations including improved klystron driver amplifiers, different comb loop phase margins, and more SLAC klystrons. 82 
5.19 Estimated damping margins at 4.5 MV for the configuration during run $5 \mathrm{~b}$ and for upgraded implementations including improved klystron driver amplifiers, different comb loop phase margins, and more SLAC klystrons. . . . . . . . . . . . . . . . . . . 83

5.20 Comparison of simulation growth rates for different gap voltage settings. 84

5.21 Comparison of estimated damping margins for different gap voltage

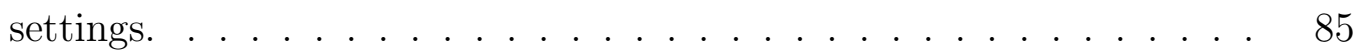

5.22 Predicted (solid lines) and measured (dashed lines) growth rates. Improvements due to the upgrades are visible. Error bars are not included for the April 2008 data because they crowd the image. They are comparable in magnitude with the error bars from the run 6 data. . . . 87

5.23 Measured improvement in growth rates versus comb rotation for LER at $2450 \mathrm{~mA} \ldots \ldots \ldots \ldots \ldots \ldots$

5.24 Measured Transfer function magnitude from LER 4-5 at $1200 \mathrm{~mA}$ showing minimal comb rotation induced distortion with the improved amplifiers. . . . . . . . . . . . . . . . .

5.25 Measured Transfer function magnitude from LER 4-2 at $1500 \mathrm{~mA}$ during run $5 \mathrm{~b}$, showing comb rotation induced distortion with the original amplifiers. . . . . . . . . . . . . . . . .

6.1 RF station transfer function based on simulated data and fitted linear model. . . . . . . . . . . . . . . . . . . . . . . . . . 94

6.2 Simplified LHC RF block diagram. . . . . . . . . . . . . 95

6.3 RF Cavern. . . . . . . . . . . . . . . . . . 96

6.4 LHC super-conducting cavity. . . . . . . . . . . . . . . 97

6.5 LHC LLRF crates. . . . . . . . . . . . . . . . . . . . 98

6.6 LHC Measured Klystron Transfer Function . . . . . . . . . . . 100

6.7 SLAC Simulated Klystron Transfer Function. ． . . . . . . . 100

6.8 LHC Measured and Simulated Open Loop RF station Transfer Function.101

6.9 LHC Measured and Simulated Closed Loop RF station Transfer Function.101 
7.1 (Color) Noise Sources. Blocks in red represent the major noise sources, in blue are the equivalent noise sources for simulations purposes, and the other components are shown in green. . . . . . . . . . . . . 105

7.2 (Color) Beam Transfer Function during physics defined by [1 0] (2 $\pi j f I-$ $A)^{-1}\left[\begin{array}{ll}0 & \omega_{s}^{2}\end{array}\right]^{T}$. The resonance is even sharper during injection (longer synchrotron radiation damping time). . . . . . . . . . . . . . . . 112

7.3 (Color) The estimated transfer function between the RF accelerating voltage phase and the noise at the q channel at the input of the modulator $\frac{\phi_{\text {cav }}(f)}{n_{q}^{M o d}(f)}(\mathrm{rad} / V)$. . . . . . . . . . . . . . . . . 118

7.4 Noise power spectral density at the output of the LHC RF Feedback for channel Q(wideband). . . . . . . . . . . . . . . . 121

7.5 Noise power spectral density at the output of the LHC RF Feedback for channel Q(narrowband). . . . . . . . . . . . . . . 121

7.6 Modal Growth Rates for configuration Injection End 0.3 A with 1-Turn Feedback (OTFB) on or off. . . . . . . . . . . . . . . . . . . 123

7.7 Tune shift for configuration Injection End 0.3 A with 1-Turn Feedback (OTFB) on or off. . . . . . . . . . . . . . . . . . . . 124

8.1 Simplified block diagram of the RF system with Beam Phase Loop. . 129

8.2 Beam 1 phase error with BPL on/off. . . . . . . . . . . . . . . 130

8.3 Beam 2 cavity sum and RF reference-BPL OFF . . . . . . . . . . . . 131

8.4 Beam 2 cavity sum with BPL On/Off . . . . . . . . . . . . . . . . 132

8.5 LHC beam response. . . . . . . . . . . . . . . . . . . . . . . . 133

8.6 Cavity phase noise for cavity 2B1 with $1.5 \mathrm{MV}$, Q of $60 \mathrm{k}, 3.5 \mathrm{TeV}$ beam.134

8.7 Cavity 6B2 noise spectral density with BPL gain. . . . . . . . . . . 135

8.8 Cavity 6B2 noise spectral density with BPL gain. . . . . . . . . . . 136

8.9 B1 Bunch Length with time. . . . . . . . . . . . . . . . . 136

8.10 B2 Bunch Length with time. . . . . . . . . . . . . . . . . . 137

8.11 RF Feedback block diagram. . . . . . . . . . . . . . . . . . . . 139

8.12 Noise at the RF Feedback Output with different switch positions. . . 140

8.13 Noise at the RF Feedback Output as a function of controller gain. . . 141 
8.14 Cavity phase power spectral density with controller gain setting from 4B2 (solid lines) and simulation (dashed lines). . . . . . . . . . . . 142

A.1 Longitudinal Feedback System. . . . . . . . . . . . . . . . . . . 149

A.2 Growth Rate Estimates from Impedance Measurements for 2 A HER. Two groups of unstable modes are expected in the 90 to $96 \mathrm{MHz}$ and 100 to $110 \mathrm{MHz}$ bands. . . . . . . . . . . . . . . . . . . . . . . . 151

A.3 Growth Rate Estimates from Impedance Measurements for 3 A LER. The two groups of unstable modes from Figure A.2 now compose a wider band from 88 to $112 \mathrm{MHz}$. A new band of unstable modes appears from 41 to $50 \mathrm{MHz}$. . . . . . . . . . . . . . . . . . . 152

A.4 HER HOM driven coupled-bunch modal growth rates for modes 790-810.153

A.5 HER HOM driven coupled-bunch modal damping rates (growth plus feedback damping). . . . . . . . . . . . . . . . . . . . 153

A.6 HER HOM driven coupled-bunch modal synchrotron frequencies. . . 154

A.7 LER HOM driven coupled-bunch modal growth rates for modes 790-810.155

A.8 LER HOM driven coupled-bunch modal damping rates (growth plus feedback damping). . . . . . . . . . . . . . . . . . . . . . . . 155

A.9 LER HOM driven coupled-bunch modal synchrotron frequencies. . . . 156

A.10 Simplified LFB Block Diagram. . . . . . . . . . . . . . . . . . 157

A.11 Beam Transfer Function - Most unstable mode. . . . . . . . . . . . . 159

A.12 10-Tap Loop Filter Transfer Function. . . . . . . . . . . . . . . . . . 159

A.13 Root Locus. . . . . . . . . . . . . . . . . . . . . . . . 160

A.14 Root Locus (zoom) . . . . . . . . . . . . . . . . . . . 161

A.15 LER noise measurements. . . . . . . . . . . . . . . . . . . 163

A.16 LER noise measurement without beam. . . . . . . . . . . . . . . . . . 164

A.17 LER noise measurements - run 6. . . . . . . . . . . . . . . . 165

A.18 HER noise measurements . . . . . . . . . . . . . . . . . 166

B.1 PEP-II estimated HOM impedance per cavity. . . . . . . . . . . . . 169

B.2 PEP-II LER $\left|R e\left(\lambda_{l}\right)\right|$ for $I_{o}=3 \mathrm{~A}$ 'by-2' fill) $\ldots \ldots$. . . . . . . . 170

B.3 PEP-II HER $\left|R e\left(\lambda_{l}\right)\right|$ for $I_{o}=2 \mathrm{~A}$ 'by-2' fill . . . . . . . . . . . . . . . 171 
B.4 Total fundamental cavity HOM impedance and third-harmonic normalconducting cavity impedance for PEP-X . . . . . . . . . . . . . . . . 172

B.5 Total fundamental cavity HOM impedance and third-harmonic superconducting cavity impedance for PEP-X . . . . . . . . . . . . . 173

B.6 PEP-X estimated $\left|R e\left(\lambda_{l}\right)\right|$, assuming 8 RF stations with 16 fundamental cavities, 19 normal-conducting third-harmonic cavities, $I_{o}=1.5 \mathrm{~A}$ and 'by-1' filling pattern. . . . . . . . . . . . . . . . . . . 173

B.7 PEP-X estimated $\left|R e\left(\lambda_{l}\right)\right|$, assuming 8 RF stations with 16 fundamental cavities, 5 super-conducting third-harmonic cavities, $I_{o}=1.5 \mathrm{~A}$ and 'by-1' filling pattern. . . . . . . . . . . . . . . . . . . 174

B.8 PEP-X estimated growth rates with a direct feedback loop. . . . . . . 175

B.9 PEP-II LER noise measurements . . . . . . . . . . . . . . . . . . 177

C.1 PEP-II Klystron Output showing the large dynamic range of operation. 180

C.2 Measuring network used to characterize the small signal behavior of the klystron driver amplifiers. . . . . . . . . . . . . . . . . . . 180

C.3 Small Signal Transfer Function of PEP-II Driver Amplifiers at a largesignal power of 10 Watts. . . . . . . . . . . . . . . . . . 181

C.4 Small Signal Transfer Function of PEP-II Driver Amplifiers at a largesignal power of 10 Watts. . . . . . . . . . . . . . . . . . . . . 182

C.5 Small Signal Transfer Function of PEP-II Driver Amplifiers at a largesignal power of 20 Watts. . . . . . . . . . . . . . . . . 183

C.6 Small Signal Transfer Function of PEP-II Driver Amplifiers at a largesignal power of 30 Watts. . . . . . . . . . . . . . . . . . . . . . 184

C.7 Intermodulation plots of PEP-II Driver Amplifiers at a large-signal power of 10 Watts. . . . . . . . . . . . . . . . . . . . . . 184

C.8 Intermodulation plots of PEP-II Driver Amplifiers at a large-signal power of 30 Watts. . . . . . . . . . . . . . . . . . . 185 


\section{Chapter 1}

\section{Introduction}

The requirements for beam intensity and energy in circular accelerators are increasing with advances in theoretical physics and new technologies that employ accelerators for other purposes. Coupled-bunch instabilities present an important source of limitations for the beam intensity in circular accelerators. As the beam intensity increases, the interaction of the beam with the surrounding resonant structures creates ever increasing wakefields. The wakefields perturb the oscillatory motion in all three directions, which can cause instability or deteriorate essential beam properties. In this dissertation we focus on the oscillations in the direction of motion - the longitudinal direction.

Historically, the coupled-bunch instabilities were mitigated with cavity detuning, lower total beam current, special fill patterns, and High-Order Mode (HOM) dampers in the accelerating cavities. As the requirements for beam intensity were further increased, feedback systems were employed to reduce the coupled-bunch instabilities. The implemented systems include impedance reducing feedback, active wideband correction, and low-mode or mode zero feedback implemented via the RF system.

It is obvious that the addition of all these feedback systems and the higher beam intensities significantly increases the complexity and the operational concerns for circular accelerators. This dissertation presents modeling and simulation methods to study and optimally configure the feedback systems, evaluate the operational options for the high-power Radio-Frequency (RF) components, and estimate the effect of 
these systems and components on longitudinal beam dynamics in circular accelerators. Both single and multi-bunch effects are considered, depending on the application.

In Chapter 2 the necessary theoretical background pertaining to beam dynamics and the RF station is introduced. The RF station-beam dynamics interaction and the employed feedback systems are introduced in Chapter 3. The motivation behind the development of the time-domain simulation is presented in Chapter 4. Of particular importance are the beam and system models used to express the RF station-beam interaction and extract the characteristics of the oscillatory motion. Chapter 4 also includes the initial validation of the simulation with data from the Positron-Electron Project II (PEP-II). Chapter 5 presents the simulation studies of the PEP-II systems with an emphasis on performance limitations and system sensitivity to LLRF parameters. Chapter 6 presents the motivation for the Large Hadron Collider (LHC) studies and describes the adaptation of the time domain simulation to the LHC implementation. The LHC simulation studies are reported in Chapter 7, and the relevant results from LHC measurements are shown in Chapter 8. Finally, Chapter 9 summarizes this work and its future directions.

A more detailed description and the main contributions of each Chapter in this dissertation are introduced in the following sections.

\subsection{Beam Dynamics and the RF station}

In order to study the beam oscillatory motion, the applicable equations of motion are developed in Chapter 2, largely following the treatment by M. Sands in [1]. The driving resonances - due to the RF cavities - and their associated wakefields are then presented. The effective impedance of these cavities and its relationship with the wakefields is introduced. The impedance of the system is next related to the beam oscillatory motion. Based on the developed beam models it is then possible to analyze the effect of the RF systems on the beam motion. 


\subsection{RF Station-Beam Dynamics Interaction}

For sufficiently high currents, the system may be unstable. Therefore, LLRF feedback systems are employed to reduce the effective impedance experienced by the beam. This reduction may still not be sufficient for some accelerators. Damping loops are then added to actively control these instabilities. Models of the RF StationBeam dynamics interaction, and the feedback techniques to mitigate and control the coupled-bunch instabilities are presented in Chapter 3.

\subsection{PEP-II Time-Domain Simulation: Motivation and Development}

The multiple feedback loops and the system sensitivity on operational choices increases the complexity of beam dynamics dependence on RF station parameters. A major incentive in the development of the time-domain simulation and related models was to provide the means to determine optimal RF configurations and study system upgrades without requiring valuable experiment time from the physical accelerator. More motivational factors and goals for this work are presented in Chapter 4.

The development of the time-domain simulation is then summarized, with an emphasis on assumptions, simplifications, and frequency domain modeling of RF components. Simulation validation data from PEP-II are presented. The validation includes transfer function and coupled-bunch instability growth rate comparisons. The tools used to identify RF parameters from an RF station closed loop transfer function and based on this information optimally configure the LLRF impedance reduction loops are also presented. 


\subsection{PEP-II Longitudinal Coupled-Bunch Instabil- ities Studies}

Simulation studies of the PEP-II longitudinal instabilities are presented in Chapter 5 . An analysis of the growth rates sensitivity on the various RF system parameters is also included. This study helped to identify performance limiting components and stability trade-offs. The resulting system upgrades and updated configurations led to substantial growth rate improvements and world record currents for PEP-II.

Based on the success of these initial studies, the longitudinal behavior at higher currents was estimated. Alternative configurations and proposed upgrades were studied to identify the biggest performance return for the investment.

\subsection{RF System Models for the LHC with Applica- tion to Longitudinal Dynamics}

The substantial effect of these studies on PEP-II motivated the adaptation of the existing time-domain simulation and related models to the LHC implementation as presented in Chapter 6. The initial simulation goals were to provide a testing tool for the development of the LHC RF configuration and optimization tools, as well as to study longitudinal beam dynamics. Validation data of the LHC simulation are also presented in this Chapter.

\subsection{LHC Longitudinal Beam Dynamics Studies}

Even though the PEP-II and LHC RF layout are very similar, the limiting beam dynamics effects are very different. The very low synchrotron radiation damping for LHC leads to an increased sensitivity to any noise presented to the beam, particularly from the RF system. A theoretical formalism relating the RF noise with longitudinal beam emittance growth is presented in Chapter 7 . With this formalism and the previously developed simulation and models, studies of the dependence of longitudinal 
emittance growth on RF configurations and noise levels were conducted. Predictions of coupled-bunch instabilities for the LHC are also presented.

\subsection{LHC Longitudinal Beam Emittance Dependence on RF Noise: Measurements}

Chapter 8 includes measurements that were conducted at the LHC to test the dependence of the longitudinal beam emittance growth on RF noise and to validate the developed formalism and models. Initial measurements showed the close correlation between the beam sampled noise power and the growth rate of the longitudinal emittance, as expected from the theoretical formalism. Through these measurements, the performance limiting RF components were determined. The data analysis also helped determine the LLRF feedback electronics that contribute mostly to RF noise.

\subsection{Summary}

The work presented in this dissertations is summarized in Chapter 9. The main contributions and potential future directions of this research are also outlined.

\subsection{Appendix}

These models and simulations can be readily adaptable to other accelerators, implementations, and beam dynamics. Some examples are included in the Appendix.

Work related to the PEP-II High-Order Mode (HOM) driven coupled-bunch instabilities is presented in Appendix A and [2]. The additional feedback and beam models for the HOM study are described. Data is presented and the effect of noise in the beam dynamics is studied.

The experience and knowledge of the RF station-beam interaction from the simulation development was also useful in the study of design concerns for PEP-X, a 
proposed light source using the PEP-II infrastructure. Operational design considerations and predictions for longitudinal stability and beam characteristics based on the conceptual parameters of the machine are presented in Appendix B.

Also reported in Appendix $\mathrm{C}$ are additional data from the PEP-II driver amplifier studies which show that the simulation accurately predicted their non-ideal behavior and distortion. Finally, Appendix D provides a short summary of essential beam and RF parameters for PEP-II and LHC. 


\section{Chapter 2}

\section{Beam Dynamics and the RF station}

This work focuses on the interaction between the beam dynamics and the RF system. Before the RF station-beam interaction is defined, the theoretical background necessary for the study of longitudinal beam dynamics, coupled-bunch instabilities and their control is presented in this Chapter. The equations of motion describing longitudinal focusing and the oscillations of the charged particles around the synchronous position are developed in Section 2.1 assuming a single-bunch ring. The analysis in Section 2.1 follows the nomenclature and ideas of M. Sands in [1], S. Prabhakar in [3], D. Teytelman in [4], A. Chao in [5], and H. Wiedemann in [6]. Section 2.2 defines the notion of wake fields which is the source of coupling among bunches. This coupling could lead to longitudinal coupled-bunch instabilities based on the configuration and parameters of the accelerator. The equations of motion in the presence of bunch cou-

pling are developed. Section 2.3 defines the longitudinal impedance, its relationship with the wake fields, and its effect on the coupling term in the equations of motion. Longitudinal impedances and coupled-bunch instabilities have also been described in [7] [8] [9] [10]. Section 2.4 describes the most common sources of impedance and the model of their contribution. 


\subsection{Single-bunch Beam Dynamics}

In circular accelerators, the magnetic guide field serves two purposes. It focuses the injected charged particles in the transverse directions (horizontal or radial and vertical), which leads to transverse oscillations around the nominal orbit, known as betatron oscillations. The magnetic guide field also directs the particle beam on the nominal orbit. The relativistic particles emit electromagnetic radiation (synchrotron radiation) due to this substantial accelerating magnetic force, which leads to energy loss over a turn. At PEP-II the average energy lost to synchrotron radiation per turn was about 3.2 MeV for the High Energy Ring (HER) and 0.6 MeV for the Low Energy Ring (LER) [11], a small fraction of the 9 and $3.1 \mathrm{GeV}$ beams respectively.

The RF system employed in a circular accelerator has three important functions: add energy to accelerate the beam, compensate for the lost energy per turn, and focus the beam in the longitudinal direction. The RF system employs multiple cavities to compensate for the average lost energy over a turn $U_{0}$. Figure 2.1 shows the RF cavity voltage $V(t)$ as a function of time. The full circles show the positions of synchronous

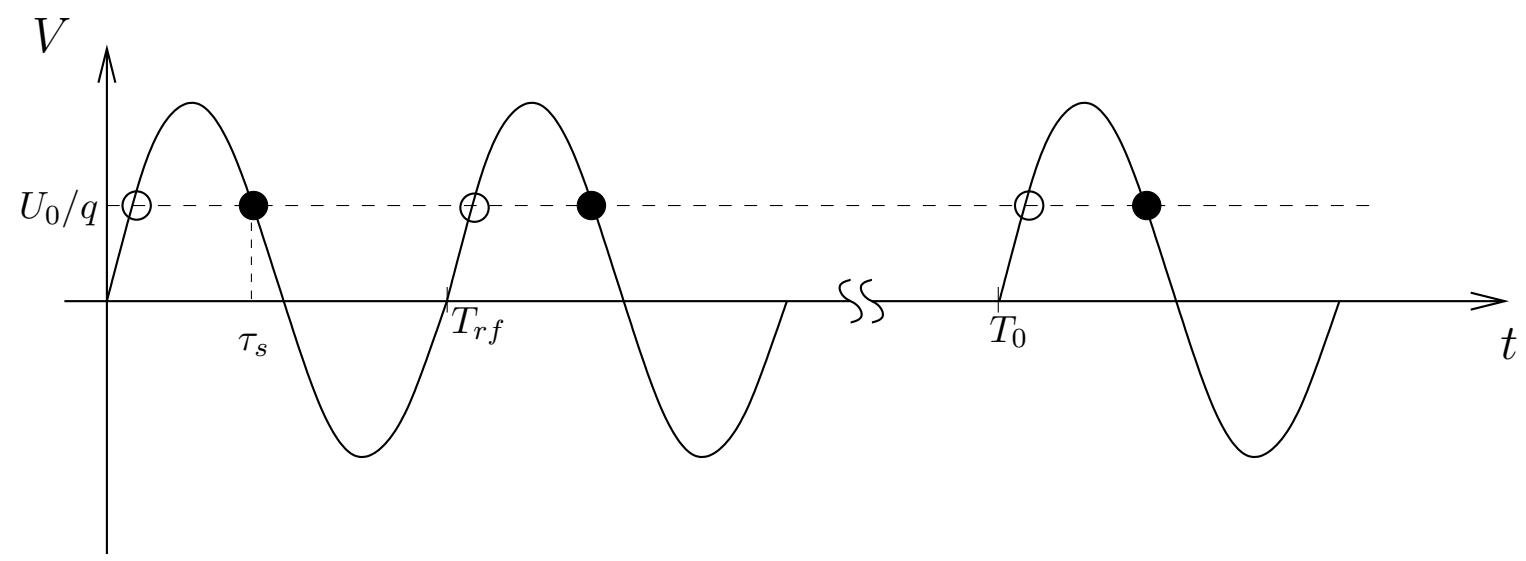

Figure 2.1: RF cavity voltage as a function of time. Filled circles represent stable equilibrium points, whereas empty circles correspond to unstable equilibrium points. 
particles - particles which follow exactly the design orbit with no oscillations over a turn - for relativistic particles, above the transition energy. $T_{\mathrm{rf}}$ is the period of the $\mathrm{RF}$ field and $T_{0}$ is the revolution period of the beam. The ratio between them is called the harmonic number $h$ (for PEP-II $h=3492$ ) and is equal to the maximum number of bunches that can be stored in a ring:

$$
h=\frac{T_{0}}{T_{\mathrm{rf}}}
$$

The synchronous particle arrives at $\tau_{s}$ and gains exactly energy $U_{0}$ at every turn.

Correct phasing of the voltage in the cavities leads to longitudinal focusing. Out of the two points per cycle for which $E(\tau)=U_{0}$, the one on the negative slope is a stable point for particles above transition. To qualitatively describe this effect, let's imagine a particle of charge $q$ arriving at the cavity a short time $\tau$ later (earlier) than the synchronous particle. The particle gains energy $E(\tau)=q V\left(\tau+\tau_{s}\right)$ which is lower (higher) than $U_{0}$, effectively moving into a smaller (larger) orbit towards the synchronous position. Therefore, a potential well is formed around the synchronous position, known as the RF bucket.

Particles in the RF bucket conduct energy oscillations around the synchronous position (the stable equilibrium point). Essentially, particles with a time advance (smaller orbits) over the synchronous position receive more energy per turn so that the time advance slowly decreases and they move to larger orbits. When the particles cross $\tau_{s}$ they have more energy than the synchronous particle, so their orbit keeps increasing, and they now start arriving later than the synchronous particle. This results in reduced energy gain per turn and the reverse correction, leading to oscillations. On the other hand, particles with a time advance (smaller orbit) over the unstable equilibrium point receive less energy per turn, moving to even smaller orbits and are eventually lost due to the finite aperture of the vacuum chamber.

To quantitatively describe the energy oscillations of the particles around the synchronous position we will need to derive the particles' equations of motion. 


\subsubsection{Equations of Motion}

Let us consider a particle of charge $q$ moving in a circular accelerator, with a path length change of $l$ corresponding to an energy deviation $\epsilon$ with respect to $E_{0}$, and a time delay $\tau$ with respect to the synchronous particle arrival time of $\tau_{s}$, as observed from a fixed point at the ring. For relativistic particles

$$
\frac{l}{L}=\frac{\tau}{T_{0}}
$$

where $L$ is the nominal orbit length. Equation 2.1 states that a relativistic particle on a longer (shorter) orbit will arrive after (before) the synchronous particle, so that $\tau$ is positive (negative). Furthermore, the momentum compaction factor $\alpha$ and the phase slip factor $\eta$ relate the path length change with the momentum and energy deviations [1]

$$
\begin{aligned}
\frac{l}{L} & =\alpha \frac{\Delta p}{p_{0}}=\left(\alpha-\frac{1}{\gamma^{2}}\right) \frac{\epsilon}{E_{0}} \\
& =\eta \frac{\epsilon}{E_{0}}
\end{aligned}
$$

where $p_{0}, E_{0}$ are the momentum and energy of the synchronous particle respectively. The momentum compaction factor (also known as dilation factor) is a dimensionless parameter that is a property of the magnetic lattice of the accelerator. The transition energy is defined as the energy when $\eta=0$. The slip factor $\eta$ is negative below transition and positive above transition. For highly relativistic beams and circular accelerators $\alpha \approx \eta>0$.

From 2.1, 2.2 the time displacement per revolution is given by

$$
\frac{\tau}{T_{0}}=\eta \frac{\epsilon}{E_{0}}
$$

and therefore the average time displacement over a turn is

$$
\frac{d \tau}{d t} \approx \frac{\tau}{T_{0}}=\eta \frac{\epsilon}{E_{0}} .
$$


The energy deviation over a turn is given by the difference of the gained energy from the RF cavities and the energy lost to synchrotron radiation:

$$
\epsilon=q V\left(\tau+\tau_{s}\right)-U_{\text {rad }}\left(\epsilon+E_{0}\right)
$$

Therefore, the average energy deviation per turn is given by

$$
\frac{d \epsilon}{d t} \approx \frac{\epsilon}{T_{0}}=\frac{q V\left(\tau+\tau_{s}\right)-U_{r a d}\left(\epsilon+E_{0}\right)}{T_{0}}
$$

Equations 2.3 and 2.4 combined give the equation of motion

$$
\ddot{\tau}-\frac{\eta}{E_{0} T_{0}}\left(q V\left(\tau+\tau_{s}\right)-U_{\text {rad }}\left(\epsilon+E_{0}\right)\right)=0
$$

From Equation 2.5, it is obvious that the equilibrium points are given by $q V\left(\tau_{s}\right)-$ $U_{\text {rad }}\left(E_{0}\right)=0$ and $\tau=0$ which corresponds to $\epsilon=0$. Since $V\left(\tau+\tau_{s}\right)$ is periodic, there are multiple equilibrium points.

\subsubsection{Equilibrium Point Stability}

The stable equilibrium points are the attractors of a local potential well as described earlier. To determine stability it is sufficient to linearize the equation of motion around the equilibrium points. Thus, for small energy deviations,

$$
\begin{aligned}
q V\left(\tau+\tau_{s}\right)-U_{\text {rad }}\left(\epsilon+E_{0}\right) & \approx q V\left(\tau_{s}\right)+\tau q \dot{V}\left(\tau_{s}\right)-U_{0}-\epsilon D \\
& =\tau q \dot{V}\left(\tau_{s}\right)-\epsilon D \\
& =\tau q \dot{V}\left(\tau_{s}\right)-\frac{E_{0} D}{\eta} \dot{\tau}
\end{aligned}
$$

where $D=\dot{U}_{\text {rad }}\left(E_{0}\right)=(2+\mathcal{D}) U_{0} / E_{0} \approx 2 U_{0} / E_{0} . \mathcal{D}$ is a function of the magnetic lattice of the accelerator and is approximately zero. 
Following the approximation from Equation 2.6, Equation 2.5 becomes

$$
\begin{aligned}
\ddot{\tau}-\frac{\eta}{E_{0} T_{0}}\left(q V\left(\tau+\tau_{s}\right)-U_{r a d}\left(\epsilon+E_{0}\right)\right) & \approx \ddot{\tau}+\frac{D}{T_{0}} \dot{\tau}-\frac{\eta q \dot{V}\left(\tau_{s}\right)}{E_{0} T_{0}} \tau \\
& =\ddot{\tau}+2 d_{r} \dot{\tau}+\omega_{s 0}^{2} \tau=0
\end{aligned}
$$

where

$$
\begin{aligned}
d_{r} & =\frac{D}{2 T_{0}} \approx \frac{U_{0}}{E_{0} T_{0}} \\
\omega_{s 0}^{2} & =-\frac{\eta q \dot{V}\left(\tau_{s}\right)}{E_{0} T_{0}} .
\end{aligned}
$$

$d_{r}$ is the radiation damping rate and $\omega_{s 0}$ is the synchrotron frequency. The synchrotron tune is defined as $\omega_{s 0} / \omega_{0}$, where $\omega_{0}=2 \pi / T_{0}$ is the revolution angular frequency. From these expressions it is obvious that for a stable equilibrium point a necessary and sufficient condition is $d_{r}>0$ and $\omega_{s 0}^{2}>0$. Under these conditions, the bunch evolves locally around the equilibrium point as a damped harmonic oscillator. To achieve these conditions for a relativistic beam above transition with $\eta>0, \dot{V}\left(\tau_{s}\right)$ has to be negative $\left(\dot{V}\left(\tau_{s}\right)\right.$ has to be positive below transition since $\left.\eta<0\right)$. This confirms our earlier statement that the high energy particles experience longitudinal focusing on the negative slope of the cavity voltage $V\left(\tau+\tau_{s}\right)$. Similarly, above transition the positive slope of the cavity voltage defines an unstable equilibrium point since $\omega_{s 0}^{2}$ is negative.

Equation 2.5 describes a system with equilibrium points at $q V\left(\tau_{s}\right)=U_{\text {rad }}\left(E_{0}\right)$. For stable equilibrium points, the particles are perturbed by quantum excitation, $\mathrm{RF}$ noise or other reasons and then follow an exponentially decaying oscillation around the synchronous position. Even though we studied the motion of a single particle, the center of mass of an RF bucket follows the same dynamics and can be described by Equation 2.5. Since in this work we will address dipole oscillations of the bunches, we will model each bunch as a macroparticle obeying the above dynamics. For multiple bunches present in the ring, the motion of one bunch can affect other bunches in the ring through coupling mechanisms that will be discussed in Section 2.2. This coupling 
can lead to longitudinal coupled-bunch instabilities.

\subsection{Coupled-bunch Dynamics}

In a circular accelerator, the RF cavity voltage $V\left(\tau+\tau_{s}\right)$ is periodic. Therefore, there are multiple stable equilibrium points, each of which corresponds to an RF bucket. Depending on the accelerator this periodic structure can be partially or fully filled with bunches. The beam dynamics is changed in these multi-bunch systems due to the coupling between the bunches induced by the vacuum chamber and RF cavities.

To understand how the vacuum chamber and RF cavities can couple the motion of bunches, we first look into its interaction with a single bunch in a circular accelerator. As the charged particles move and interact with the vacuum chamber and the cavities, they leave behind electromagnetic fields [8], known as wake fields. Since the wake fields created by these parasitic resonances can have damping times of hundreds of revolutions, the bunch could be affected by the fields when it returns one revolution period later.

For a multi-bunch system, the coupling is more critical since the time between bunches is lower than the revolution period $T_{0}$ by the harmonic number $h$. Any perturbation in the time of arrival of a single bunch, will now modulate the wake fields seen by the trailing bunches. Therefore, the wake fields couple the motion of bunches. For certain resonant frequencies and sufficiently high bunch current, the system could become unstable (coupled-bunch instabilities).

Assume a test charge $q$ traversing the vacuum chamber $t$ seconds after a unit charge. The longitudinal wake function $W^{\|}(t)$ is defined as the longitudinal component of this electric field experienced by the test charge over the whole ring. From this definition and causality, $W^{\|}(t)=0$ for $t<0$.

To calculate the wake field voltage $V_{n}^{w k}(t)$ experienced by the $n^{t h}$ bunch at time $t$, we need to integrate the wake function over all preceding turns and bunches:

$$
V_{n}^{w k}(t)=\sum_{p=-\infty}^{\infty} \sum_{k=0}^{N-1} q_{k} W^{\|}\left((p N+n-k) T_{b}+\tau_{n}(t)-\tau_{k}\left(t-(p N+n-k) T_{b}\right)\right)
$$


where $q_{k}$ is the charge of bunch $k$ preceding bunch $n$ by $p$ turns, $N$ is the number of bunches, and $T_{b}$ is the bunch spacing $\left(T_{b}=T_{o} / N\right.$, which is not necessarily equal to $T_{\text {rf }}$ depending on the filling pattern).

If we assume an equal charge $q$ for all bunches,

$$
V_{n}^{w k}(t)=q \sum_{u=-\infty}^{\infty} W^{\|}\left(u T_{b}+\tau_{n}(t)-\tau_{u}\left(t-u T_{b}\right)\right)
$$

where $u T_{b}=(p N+n-k) T_{b}$ is the spacing between the $k^{\text {th }}$ and $n^{\text {th }}$ bunches $p$ turns apart.

The longitudinal wake field voltage is added to $V\left(\tau+\tau_{s}\right)$ in the equation of motion - Equation 2.5 - which for the $n^{\text {th }}$ bunch becomes

$$
\ddot{\tau}_{n}-\frac{\eta}{E_{0} T_{0}}\left(q V\left(\tau_{n}+\tau_{s}\right)+q V_{n}^{w k}(t)-U_{\text {rad }}\left(\epsilon+E_{0}\right)\right)=0
$$

where $q V_{n}^{w k}(t) / T_{0}$ is the rate of energy change for the $n^{t h}$ bunch due to the wake fields created by all preceding bunches.

At the equilibrium points, $\tau_{n}=\tau_{u}=0$ and $\epsilon=0$, so that

$$
V_{n}^{w k}\left(\tau_{s}\right)=q \sum_{u=-\infty}^{\infty} W^{\|}\left(u T_{b}\right)
$$

and the new equilibrium is given by

$$
q V\left(\tau_{s}\right)+q^{2} \sum_{u=-\infty}^{\infty} W^{\|}\left(u T_{b}\right)=U_{\text {rad }}\left(E_{0}\right)=U_{0} .
$$

The coupled-bunch interaction introduces a shift in the equilibrium position $\tau_{s}$ or equivalently a shift in the synchronous phase with respect to the single-bunch phase, with the corresponding effect on beam dynamics. For the rest of this work, $\tau_{s}$ will refer to the equilibrium point corresponding to the coupled-bunch dynamics. 


\subsubsection{Equilibrium Point Stability}

As in Section 2.1, by linearizing Equation 2.8 around the defined equilibrium point we can determine the system's stability and quantify the dynamic interaction between the beam and the fundamental longitudinal impedance for an even-fill beam pattern. The following analysis is for a general periodic cavity voltage $V\left(\tau+\tau_{s}\right)$, even though for the circular accelerators studied in this work the cavity voltage is sinusoidal $V\left(\tau+\tau_{s}\right)=$ $V_{0} \sin \left(\omega_{\mathrm{rf}}\left(\tau+\tau_{s}\right)\right)=V_{0} \sin \left(\omega_{\mathrm{rf}} \tau+\phi_{s}\right)$.

The time displacements $\tau$ are typically much smaller than $T_{\mathrm{rf}}$, so we can approximate

$$
W^{\|}\left(u T_{b}+\tau_{n}(t)-\tau_{u}\left(t-u T_{b}\right)\right) \approx W^{\|}\left(u T_{b}\right)+\left(\tau_{n}(t)-\tau_{u}\left(t-u T_{b}\right)\right) \dot{W}^{\|}\left(u T_{b}\right)
$$

using the Taylor Expansion.

With the above assumptions, Equations 2.3, 2.10, and for small energy deviations,

$$
\begin{gathered}
q V\left(\tau_{n}+\tau_{s}\right)+q V_{n}^{w k}(t)-U_{\text {rad }}\left(\epsilon+E_{0}\right) \approx \\
\approx q V\left(\tau_{s}\right)+\tau_{n} q \dot{V}\left(\tau_{s}\right)+q^{2} \sum_{u=-\infty}^{\infty}\left(W^{\|}\left(u T_{b}\right)+\left(\tau_{n}(t)-\tau_{u}\left(t-u T_{b}\right)\right) \dot{W}^{\|}\left(u T_{b}\right)\right)-U_{0}-\epsilon D \\
=\tau_{n} q \dot{V}\left(\tau_{s}\right)-\epsilon D+q^{2} \sum_{u=-\infty}^{\infty}\left(\tau_{n}(t)-\tau_{u}\left(t-u T_{b}\right)\right) \dot{W}^{\|}\left(u T_{b}\right) \\
=\tau_{n} q \dot{V}\left(\tau_{s}\right)-\frac{E_{0} D}{\eta} \dot{\tau}_{n}+q^{2} \sum_{u=-\infty}^{\infty}\left(\tau_{n}(t)-\tau_{u}\left(t-u T_{b}\right)\right) \dot{W}^{\|}\left(u T_{b}\right)
\end{gathered}
$$

where $D=\dot{U}_{\text {rad }}\left(E_{0}\right)=(2+\mathcal{D}) U_{0} / E_{0} \approx 2 U_{0} / E_{0}$.

Following the simplifications from 2.12, Equation 2.8 becomes:

$$
\ddot{\tau}_{n}-\frac{\eta}{E_{0} T_{0}}\left(\tau_{n} q \dot{V}\left(\tau_{s}\right)-\frac{E_{0} D}{\eta} \dot{\tau}_{n}+q^{2} \sum_{u=-\infty}^{\infty}\left(\tau_{n}(t)-\tau_{u}\left(t-u T_{b}\right)\right) \dot{W}^{\|}\left(u T_{b}\right)\right)=0
$$


which can be rewritten as

$$
\ddot{\tau}_{n}-\frac{\eta}{E_{0} T_{0}}\left(\tau_{n} q \dot{V}\left(\tau_{s}\right)-\frac{E_{0} D}{\eta} \dot{\tau}_{n}\right)=-\frac{\eta q^{2}}{E_{o} T_{o}} \sum_{u=-\infty}^{\infty}\left(\tau_{n}(t)-\tau_{u}\left(t-u T_{b}\right)\right) \dot{W}^{\|}\left(u T_{b}\right)
$$

or equivalently,

$$
\ddot{\tau}_{n}+2 d_{r} \dot{\tau}_{n}+\omega_{s}^{2} \tau_{n}=-\frac{\eta q^{2}}{E_{o} T_{o}} \sum_{u=-\infty}^{\infty}\left(\tau_{n}(t)-\tau_{u}\left(t-u T_{b}\right)\right) \dot{W}^{\|}\left(u T_{b}\right)
$$

where

$$
\begin{aligned}
d_{r} & =\frac{D}{2 T_{0}} \approx \frac{U_{0}}{E_{0} T_{0}} \\
\omega_{s}^{2} & =-\frac{\eta q \dot{V}\left(\tau_{s}\right)}{E_{0} T_{0}} .
\end{aligned}
$$

It is important to note that $\omega_{s} \neq \omega_{s 0}$. From these expressions it is obvious that the motion of all bunches couples to the motion of bunch $\tau_{n}$ through the right hand side of Equation 2.13.

\subsubsection{Third-Harmonic Cavity Example}

To illustrate the shift in the equilibrium position $\tau_{s}$ an example of the effect of a ThirdHarmonic cavity is included here. Passive Third-Harmonic cavities are often used in light sources to increase the bunch length. As shown in Figure 2.2 the introduction of the Third-Harmonic cavity shifts the equilibrium position, since the energy $U_{0}$ is now compensated at a different phase. Furthermore, the decrease in the absolute value of the accelerating voltage around the equilibrium position $\dot{V}\left(\tau_{s}\right)$ reduces the synchrotron frequency according to Equation 2.14. The bunch length $\sigma_{z}$ is given by

$$
\sigma_{z}=\frac{\eta c}{\omega_{s}}\left(\frac{\sigma_{E}}{E_{o}}\right)
$$

where $\frac{\sigma_{E}}{E_{o}}$ is the energy spread. As a result, the bunch length is increased due to the new slope of the accelerating voltage. Figure 2.2 approximates the relative amplitude 


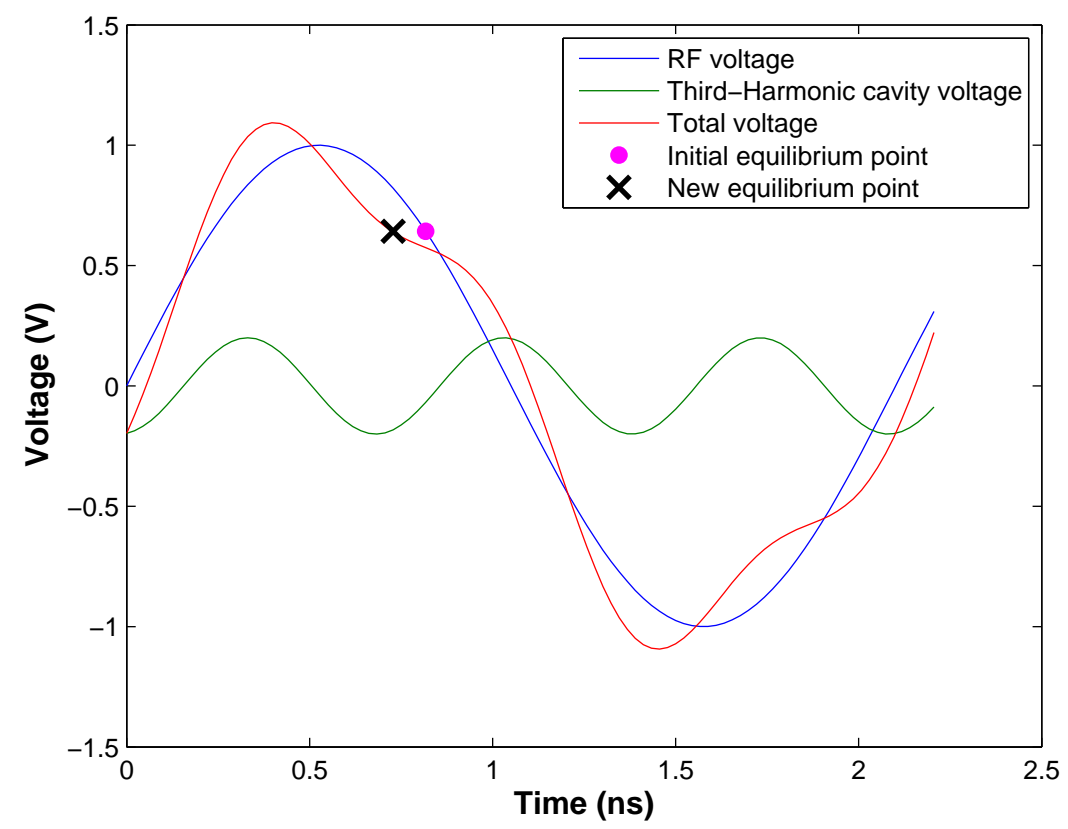

Figure 2.2: Equilibrium point shift and voltage slope change with addition of ThirdHarmonic cavity.

of the fundamental and Third-Harmonic cavity voltages for PEP-X. With a fundamental voltage of $10 \mathrm{MV}$, the synchrotron frequency is approximately $1 \mathrm{kHz}$ and the bunch length $2.5 \mathrm{~mm}$. The addition of a 2.35 MV Third-Harmonic cavity halves the synchrotron frequency to $500 \mathrm{~Hz}$ and doubles the bunch length to $5 \mathrm{~mm}$. It should be noted that depending on the phase of the Third-Harmonic cavity, the slope of the accelerating voltage can be increased or decreased, with the corresponding effect on bunch length.

\subsubsection{Modal Domain}

Assuming that the modal eigenvalues are close to the imaginary axis $\left(\lambda_{l} \approx j \omega_{l}\right)$, Equation 2.13 has solutions of the form $\tau_{n}=e^{\lambda t}$ or $\tau_{n}=e^{j \omega_{l} t}$. Exploiting the ring symmetry, we choose the basis $e^{j 2 \pi l / N}$ for $l \in[0, N-1]$. With our choice of a basis, it is more useful to express the time deviation of the $n^{t h}$ bunch as the phase deviation at the RF frequency, $\phi_{n}=\omega_{\mathrm{rf}} \tau_{n}$. The phase deviation $\phi_{n}$ of the $n^{\text {th }}$ bunch in the 
bunch domain can then be transformed to the $N$ even-filled bunch base by

$$
\begin{aligned}
\varphi_{l}(t) & =\frac{1}{N} \sum_{n=0}^{N-1} \phi_{n}(t) e^{-i 2 \pi l n / N} \\
& =\frac{\omega_{\mathrm{rf}}}{N} \sum_{n=0}^{N-1} \tau_{n}(t) e^{-i 2 \pi l n / N}
\end{aligned}
$$

where $\varphi_{l}$ is the phase deviation of the $l^{\text {th }}$ mode in the eigenmodal domain.

Using $2.15,2.13$ is given by:

$$
\ddot{\varphi}_{l}+2 d_{r} \dot{\varphi}_{l}+\omega_{s}^{2} \varphi_{l}=-\varphi_{l} \frac{\eta q^{2}}{E_{o} T_{o}} \sum_{u=-\infty}^{\infty}\left(1-e^{-j\left(l \omega_{0}+\omega_{l}\right) u T_{b}}\right) \dot{W}^{\|}\left(u T_{b}\right) .
$$

\subsection{Introducing Impedances}

From the definition in Section 2.2, the wake function $W^{\|}(t)$ is the impulse response of the accelerating structure to a unit charge. The longitudinal impedance is defined as the frequency response of the accelerating structure to that same charge [5]. Therefore, $W^{\|}(t)$ is the inverse Fourier Transform of the longitudinal impedance

$$
W^{\|}(t)=\frac{1}{2 \pi} \int_{-\infty}^{+\infty} Z^{\|}(\omega) e^{j \omega t} d \omega
$$

In the frequency domain, the longitudinal impedance is the transfer function between the beam current and the wake field voltage:

$$
Z^{\|}(\omega)=\frac{V^{w k}(\omega)}{I(\omega)}
$$

Since the wake function is real, the longitudinal impedance is hermitian, a useful fact for the analysis of impedances in the frequency domain

$$
Z^{\|}(\omega)=\left(Z^{\|}(-\omega)\right)^{*}
$$


Using Poisson's summation formula and Fourier identities, 2.16 can be transformed to

$$
\begin{aligned}
& \ddot{\varphi}_{l}+2 d_{r} \dot{\varphi}_{l}+\omega_{s}^{2} \varphi_{l}= \\
& \quad-\varphi_{l} \frac{j \eta q I_{0}}{E_{o} T_{o}} \sum_{p=-\infty}^{\infty}\left(p N \omega_{0} Z^{\|}\left(p N \omega_{0}\right)-\left((p N+l) \omega_{0}+\omega_{l}\right) Z^{\|}\left((p N+l) \omega_{0}+\omega_{l}\right)\right)
\end{aligned}
$$

where $I_{0}=q / T_{b}$ is the average (DC) beam current.

If $d_{r} \ll \omega_{s}$, the left hand side of Equation 2.17 can be further simplified,

$$
\ddot{\varphi}_{l}+2 d_{r} \dot{\varphi}_{l}+\omega_{s}^{2} \varphi_{l} \approx 2 j \omega_{s}\left(\dot{\varphi}_{l}+\left(d_{r}-j \omega_{s}\right) \varphi_{l}\right)
$$

This condition essentially limits the maximum bunch coupling for which this representation is accurate. For all the cases presented in the dissertation this criterion is well satisfied. This simplification leads to

$$
\begin{aligned}
& \dot{\varphi}_{l}+\left(d_{r}-j \omega_{s}\right) \varphi_{l}= \\
& -\varphi_{l} \frac{\eta q I_{0}}{2 E_{o} T_{o} \omega_{s}} \sum_{p=-\infty}^{\infty}\left(p N \omega_{0} Z^{\|}\left(p N \omega_{0}\right)-\left((p N+l) \omega_{0}+\omega_{l}\right) Z^{\|}\left((p N+l) \omega_{0}+\omega_{l}\right)\right)
\end{aligned}
$$

Finally, we define the total effective longitudinal impedance $Z^{\| \text {eff }}(\omega)$ as

$$
Z^{\| \mathrm{eff}}(\omega)=\frac{1}{\omega_{\mathrm{rf}}} \sum_{p=-\infty}^{\infty}\left(p N \omega_{o}+\omega\right) Z^{\|}\left(p N \omega_{o}+\omega\right)
$$

to get the final expression for the equation of motion of mode $l$ in the presence of longitudinal bunch coupling:

$$
\dot{\varphi}_{l}+\left(d_{r}-j \omega_{s}\right) \varphi_{l}=\frac{\eta q I_{0} \omega_{\mathrm{rf}}}{2 E_{o} T_{o} \omega_{s}}\left(Z^{\| \mathrm{eff}}\left(l \omega_{0}+\omega_{l}\right)-Z^{\| \mathrm{eff}}(0)\right) \varphi_{l}=\lambda_{l} \varphi_{l}
$$

where $\omega_{l}$ is the oscillation frequency of mode $l$.

This expression is of the form $\dot{\varphi}_{l}=\Lambda_{l} \varphi_{l}$ where $\Lambda_{l}=\lambda_{l}-d_{r}+j \omega_{s}=\sigma_{l}+j \omega_{l}$ is the complex natural frequency. Assuming $\omega_{s} \approx \omega_{l}$, the modal growth rate of the $l^{\text {th }}$ 
characteristic beam mode $\sigma_{l}$ and the modal oscillation frequency $\omega_{l}$ are then given by

$$
\begin{aligned}
& \sigma_{l} \approx-d_{r}+\frac{\eta q I_{0} \omega_{\mathrm{rf}}}{2 E_{o} T_{o} \omega_{s}} \mathcal{R}\left(Z^{\| \mathrm{eff}}\left(l \omega_{0}+\omega_{s}\right)-Z^{\| \mathrm{eff}}(0)\right) \\
& \omega_{l} \approx \omega_{s}+\frac{\eta q I_{0} \omega_{\mathrm{rf}}}{2 E_{o} T_{o} \omega_{s}} \mathcal{I}\left(Z^{\| \mathrm{eff}}\left(l \omega_{0}+\omega_{s}\right)-Z^{\| \mathrm{eff}}(0)\right) .
\end{aligned}
$$

Equation 2.20 defines the eigenvalues of the beam dynamics in the beam modal frame. The effect of the longitudinal effective impedance is evident on the modal damping and the deviation of the synchrotron frequency of the individual modes from $\omega_{s}$. It is important to note that for zero beam current $\sigma_{l}$ is negative - the eigenvalues are decaying transients. As the beam current $I_{o}$ increases though, the impedance term will eventually become larger than $d_{r}$ and $\sigma_{l}$ would be positive and the modal motion is growing. The threshold current was approximately $150 \mathrm{~mA}$ for PEP-II.

\subsection{Sources of Impedance}

The most significant sources of longitudinal impedance are the fundamental and higher order modes of the RF cavities. Each RF resonant cavity mode can be modeled as a resistor/inductor/capacitor (RLC) parallel circuit. The impedance contribution from each resonant mode is then given by

$$
\begin{aligned}
Z_{i}^{\|}(\omega) & =\frac{1}{\frac{1}{R_{i}}+\frac{1}{j \omega L_{i}}+j \omega C_{i}} \\
& =\frac{R_{i}}{1+Q\left(\omega_{r i} / s+s / \omega_{r i}\right)} \\
& =\frac{2 R_{i} \sigma_{i}}{s^{2}+2 \sigma_{i} s+\omega_{r i}^{2}}
\end{aligned}
$$

where $s=j \omega, R_{i}$ is the shunt impedance, $Q_{i}=R_{i} \sqrt{\frac{C_{i}}{L_{i}}}$ is the quality factor, $\omega_{r i}=$ $\frac{1}{\sqrt{L_{i} C_{i}}}$ is the resonant angular frequency of mode $i$, and $\sigma_{i}=\omega_{r i} s / 2 Q_{i}$.

During the PEP-II design, Bob Rimmer measured the center frequency, shunt impedance and quality factor for the HOM resonances in a prototype cavity [12]. Using this data and Equation 2.21, we can sum the impedances of all the longitudinal 
HOM modes to reconstruct the longitudinal HOM impedance of the cavity. An estimate of the HOM longitudinal impedance magnitude per cavity for PEP-II is

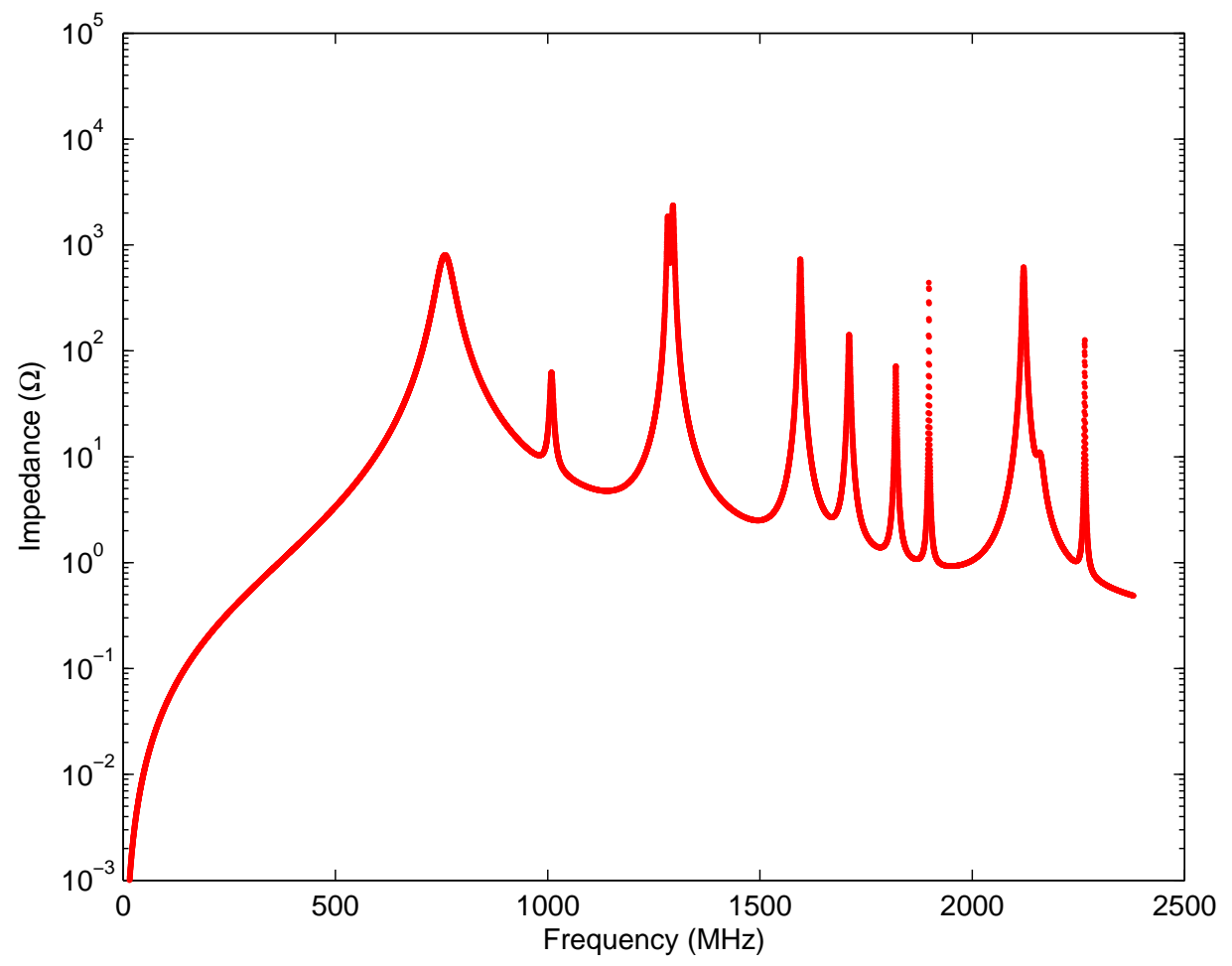

Figure 2.3: PEP-II estimated longitudinal HOM impedance per cavity, $\sum Z_{i}^{\|}$.

shown in Figure 2.3.

The cavity fundamental mode impedance is much higher than the impedance presented by the HOM. Therefore, in this dissertation the focus is on coupled-bunch instabilities driven by the cavity fundamental. It should also be noted that there are other longitudinal effects perturbing the wake fields, such as head-tail effects. These effects are not included in this dissertation, since the coupling wake fields are in very high frequencies and as such do not interfere with the the cavity fundamental driven modes. 


\section{Chapter 3}

\section{RF Station-Beam Dynamics Interaction}

The RF station-beam interaction is of fundamental importance for this work. Figure 3.1 presents a simplified diagram of this interaction. To create the accelerating voltage $V$, a current $I_{T}$ is driven through the accelerating cavity fundamental impedance - modeled by a RLC circuit in parallel. A generator (klystron) drives the current. LLRF feedback loops are then added to regulate/control the accelerating voltage. The beam energy is modulated by the cavity voltage, thus affecting the beam dynamics. In turn, the modulated beam current $I_{B}$ impacts the accelerating voltage. Therefore, there are two loops around the accelerating cavity; the LLRF loop and the beam dynamics loop. These two systems are coupled leading to coupled-bunch instabilities. In Section 2.3, the quantitative dependence of the beam dynamics on the RF station impedance was presented. Equation 2.19 links the RF station impedance with the beam dynamics.

The LLRF system also reduces the cavity fundamental impedance experienced by the beam, but the group delay in the LLRF feedback caps the impedance reduction. For sufficiently high beam current, the beam can still be unstable. Damping systems are then employed to control the instabilities. This successive shift of the system's dominant eigenvalue from the unstable right half of the complex plane to stability is depicted qualitatively in Figure 3.2. Budgeting between damping and impedance 


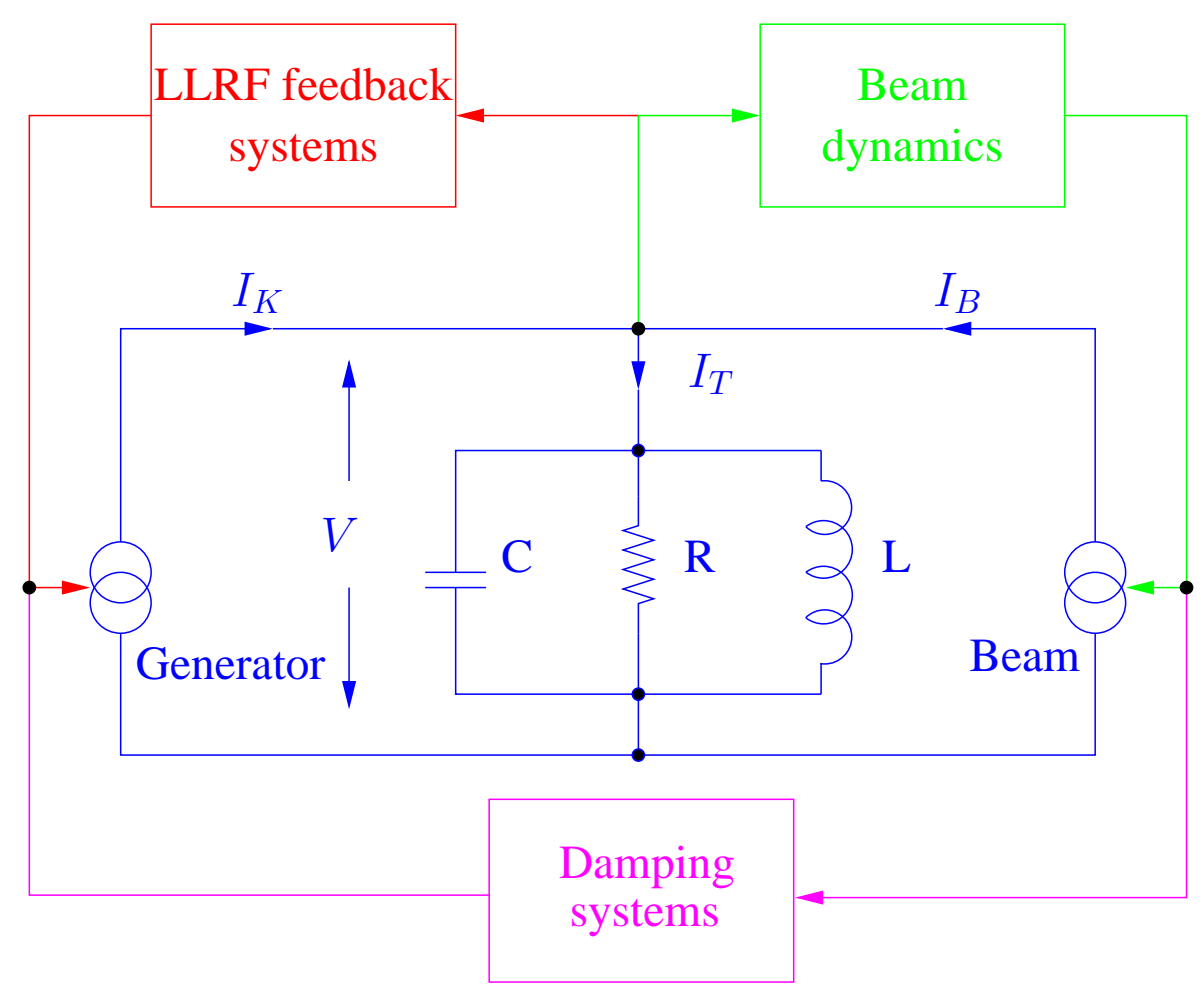

Figure 3.1: RF station-beam interaction through the cavity impedance.

reduction is essential for machine performance. The ratio varies among machines.

To achieve stability, both the beam and the LLRF loops have to be stable. The analysis of the system stability is complicated due to the non-linear nature of the components and the RF station-beam interaction through the cavity impedance. It is our goal with this work to simulate, model, and better understand this interaction, so that we can determine optimal configurations, estimate system sensitivity to components, evaluate the technical limitations, and propose alternatives and upgrades.

Section 3.1 presents the Pedersen model of the RF station-beam interaction and Section 3.2 contains a more detailed overview of the feedback techniques to mitigate and control the couple-bunch instabilities. 


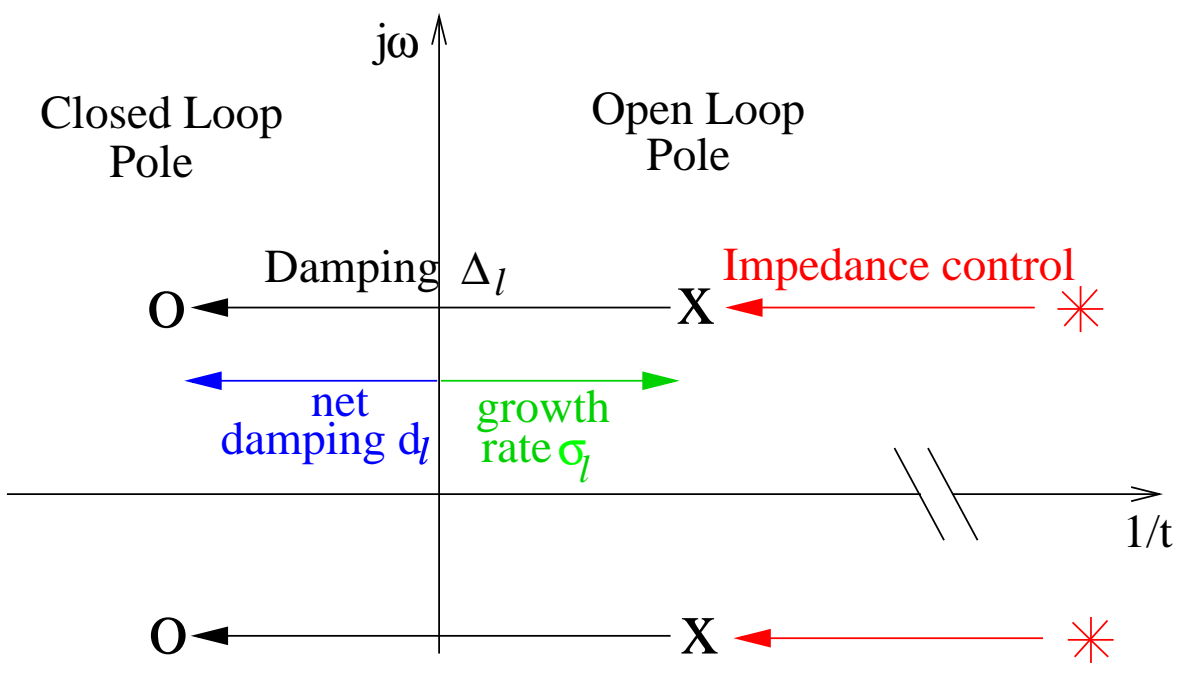

Figure 3.2: Representation of the system's pole with LLRF and damping loops (closed loop pole o), the pole with the LLRF feedback (open loop pole $\mathrm{x}$ ), and in the absence of any feedback $($ red $*)$.

\subsection{Pedersen Model of the RF Station-Beam In- teraction}

Before presenting the Feedback Control systems employed to reduce coupled-bunch instabilities, it is important to relate the equation of motion given by Equation 2.19 with the widely used Pedersen model of the RF station-beam interaction [9], [13], [14]. The Pedersen model studies the interaction of the beam with the fundamental cavity mode impedance. The development of feedback systems in circular accelerators with multiple cavities was a major motivation for the development of this model. Most high energy physics accelerators employ multiple cavities. Therefore, the total accelerating voltage is generated by the vector sum of the individual cavity voltages and thus is a function of the impedances and klystron current for each individual cavity. Even though the equations of motion developed in Chapter 2 correctly describe the motion of the particles given the total cavity voltage, the design and control of feedback systems developed for each RF station required a different formalism, the Pedersen model.

Due to the periodicity of the stable equilibrium points and assuming a uniform fill 
pattern, all bunches experience the same wake field voltage in steady state. The wake field voltage experienced by bunch $n$ was defined in Equation 2.7. Let's consider the generic observation time $t=\tau_{n}+\tau_{s}$ and assume that the ring is in steady state so that $\tau_{i}=0$ for all the bunches. Then, the wake field $V^{w k}(t)$ becomes

$$
\begin{aligned}
V^{w k}(t) & =q \sum_{u=-\infty}^{\infty} W^{\|}\left(u T_{b}+t-\tau_{s}\right) \\
& =q W^{\|}(t) * \sum_{u=-\infty}^{\infty} \delta\left(t+u T_{b}-\tau_{s}\right)
\end{aligned}
$$

Taking the Fourier transform of this expression results in

$$
\begin{aligned}
V^{w k}(f) & =Z^{\|}(f) \frac{q}{T_{b}} \sum_{u=-\infty}^{\infty} \delta\left(f+\frac{u}{T_{b}}\right) e^{-2 \pi j \tau_{s} f} \\
& =\left(Z_{0}^{\|}(f)+Z_{H O M}^{\|}(f)\right) I_{B_{T}}(f) .
\end{aligned}
$$

where $Z_{0}^{\|}(f)$ is the impedance due to the the fundamental cavity mode and $Z_{H O M}^{\|}(f)$ is the total impedance due to the high-order cavity modes.

Since $V\left(\tau+\tau_{s}\right)=V_{0} \sin \left(\omega_{R F} \tau+\phi_{s}\right)$, the generator (klystron) interacts only with the fundamental impedance at $\omega_{R F}$ presenting a voltage $V(f)=I_{K}(f) Z_{0}^{\|}(f)$, where $I_{K}$ is the klystron current $\left(Z_{H O M}^{\|}\right.$does not interact with signals at $\left.\omega_{R F}\right)$.

Therefore, the total voltage interacting with the periodic beam is given by:

$$
\begin{aligned}
V(f)+V^{w k}(f) & =I_{K}(f) Z_{0}^{\|}(f)+\left(Z_{0}^{\|}(f)+Z_{H O M}^{\|}(f)\right) I_{B_{T}}(f) \\
& =\left(I_{B_{T}}(f)+I_{K}(f)\right) Z_{0}^{\|}(f)+I_{B_{T}}(f) Z_{H O M}^{\|}(f) \\
& \approx\left(I_{B}(f)+I_{K}(f)\right) Z_{0}^{\|}(f)+I_{B_{T}}(f) Z_{H O M}^{\|}(f) .
\end{aligned}
$$

The last approximation assumes that the contribution on the accelerating voltage of $I_{B_{T}}(f) Z_{0}^{\|}(f)$ is dominated by the harmonic around $f_{R F}$, denoted $I_{B}(f)$.

The total interaction is shown in Figure 3.3, from which it is easy to see that the total current interacting with the cavity fundamental mode is $I_{T}=I_{B}+I_{K}$. The voltage across $Z_{0}$ is the total cavity voltage $V(f)=\left(I_{K}(f)+I_{B}(f)\right) Z_{0}^{\|}$. The voltage 


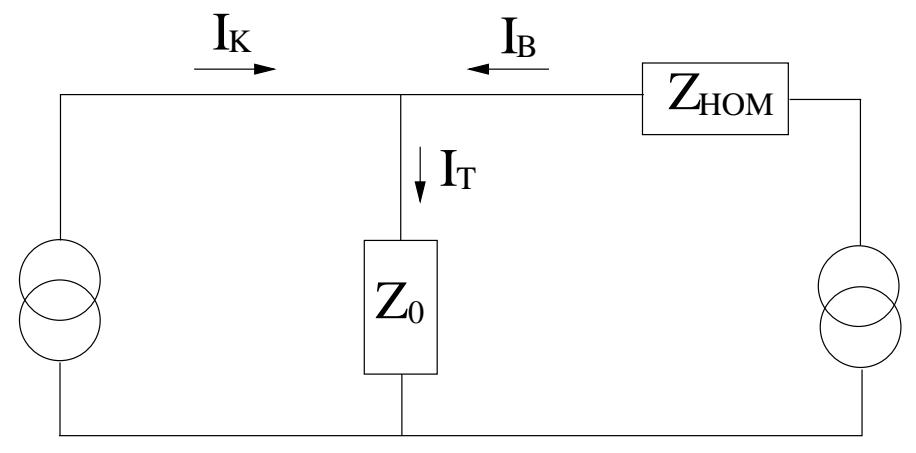

Figure 3.3: Pedersen model block diagram.

across $Z_{H O M}^{\|}$corresponds to the losses due to the high-order mode impedance, which have been shown to be a small fraction of the synchrotron radiation losses [11] and are not included in the Pedersen model.

Based on this model, and the discussion in Sections 2.2, 2.3, the coupled-bunch dynamics can be thought of as a feedback system including the longitudinal impedance and the beam, as simplistically shown in Figure 3.4. As shown above, the current

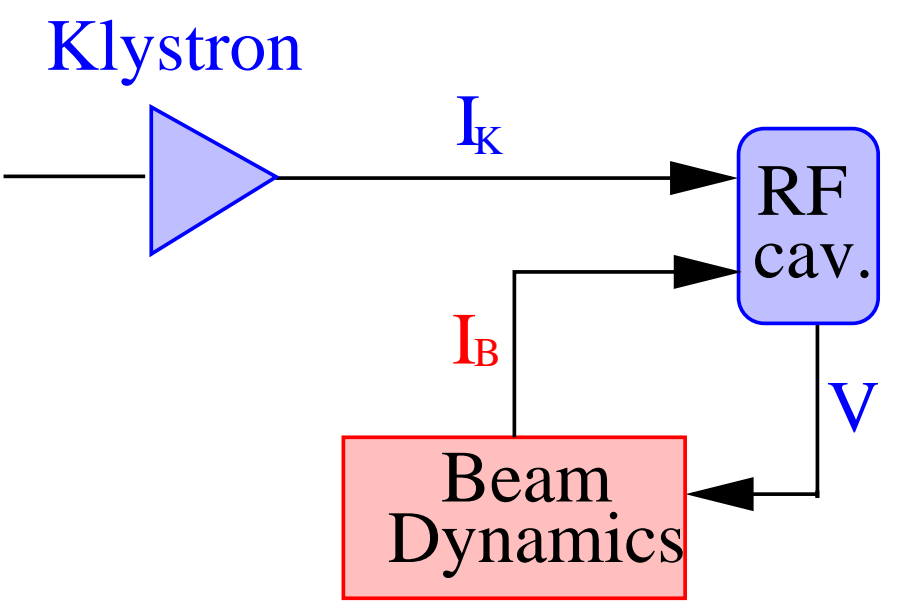

Figure 3.4: Block diagram representation of the RF station-beam interaction.

experienced by the RF cavity is the sum of the generator and beam currents. This relationship is depicted graphically in Figures 3.5 and 3.6. In the case of low beam currents (light beam loading), the generator current dominates, and so the RF stationbeam interaction is weak. If though the beam current is comparable to the generator 


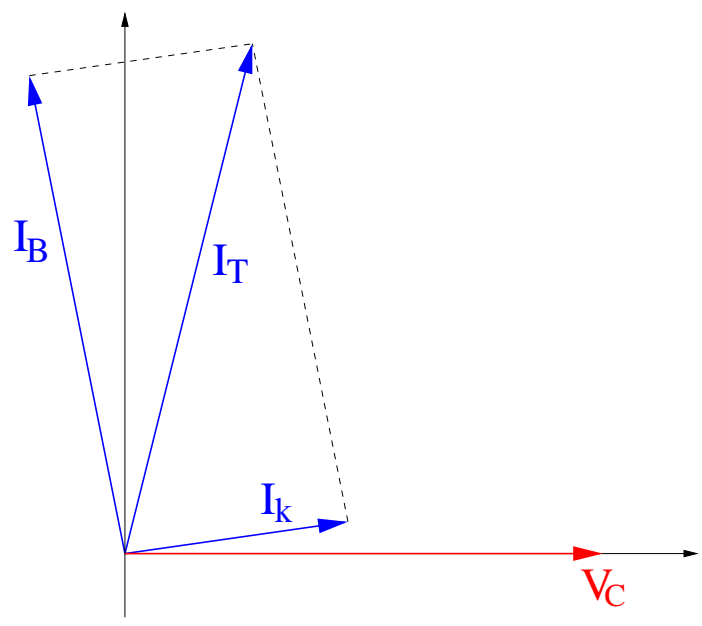

Figure 3.5: Phasor Diagram at $\omega_{R F}$ of the RF station-beam interaction heavy beam loading.

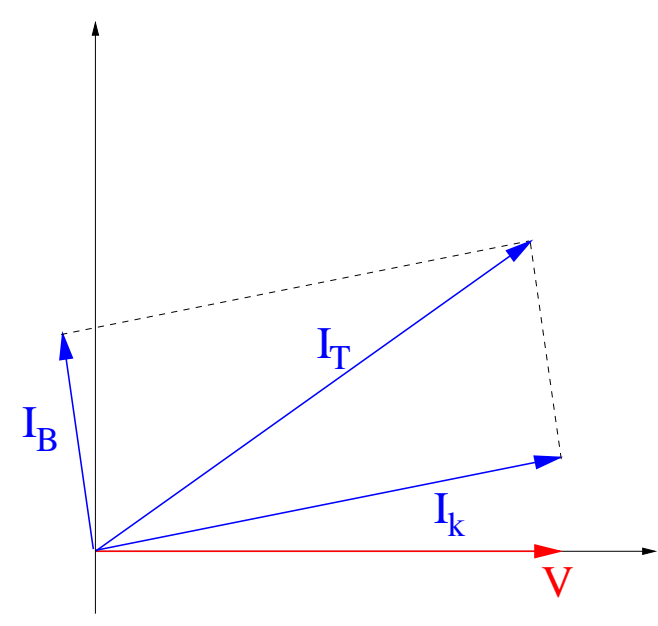

Figure 3.6: Phasor Diagram at $\omega_{R F}$ of the RF station-beam interaction - light beam loading.

current (heavy beam loading), there is a strong RF station-beam interaction. As a consequence, both the beam dynamics, but also the dynamics of the RF station are affected. Furthermore, heavy-beam loading can lead to longitudinal instability and beam loss. For PEP-II, beam loading was heavy enough that the worst case instability growth time constant was about a fifth of the synchrotron period.

\subsection{Feedback Control Systems}

To reduce these coupled-bunch instabilities, changes in beam parameters can be effective. An increase of synchrotron radiation (by an increase in beam energy), a smaller number of bunches, or a different oscillation frequency can achieve stability. Landau damping (a spread of the synchrotron frequencies in a bunch or over all bunches in the ring), can also be effective [3], [15], [16]. These beam characteristics are closely related with the experiment served by each circular accelerator though, and are often times fixed. Therefore, passive techniques for lowering the impedance and active systems for the control of coupled-bunch instabilities have been developed [17], [18]. 


\subsubsection{Passive Cures}

Impedance minimization is often employed to reduce coupled-bunch instabilities [19]. Reducing the impedance experienced by the beam lowers the coupling between successive bunches and the longitudinal instabilities. There are various ways to reduce the ring's impedance. The geometry [20] and materials of the cavities are an important consideration. The HOM impedances that cannot be attenuated by careful design are then damped through the selection of waveguides and loads [21]. A PEP-II cavity is shown in Figure 3.7 with the rectangular ports for the damping waveguides clearly visible. The folded waveguides are shown in Figure 3.8 on an installed cavity. The

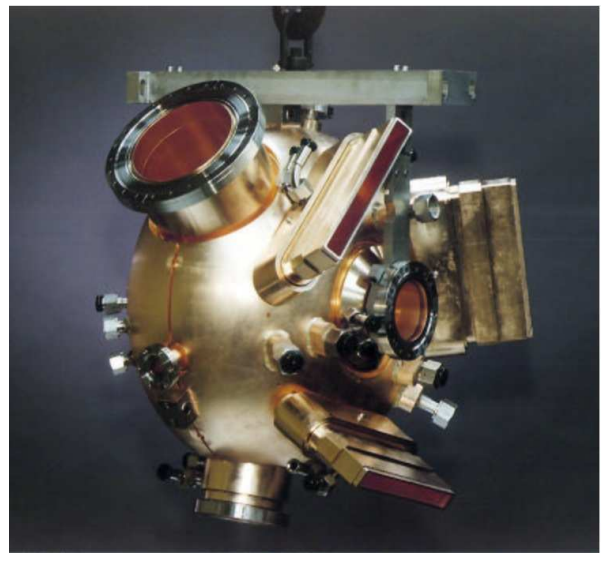

Figure 3.7: PEP-II cavity with rectangular waveguide ports.

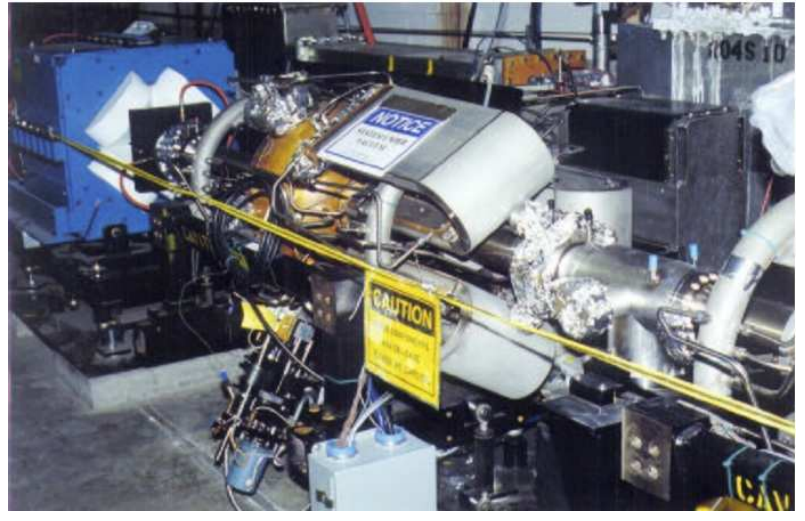

Figure 3.8: Installed PEP-II cavity with damping waveguides.

effect of residual HOM impedances of high Q is minimized by tuning their resonant frequencies in between revolution harmonics [22], as evident by Equation 2.20. On the other hand, damped HOM impedances of low Q span multiple revolution harmonics and therefore cannot be tuned. A similar scheme is used for the cavity fundamental. The cavity is detuned below the RF frequency to achieve Robinson stability (stability of mode 0) [23], [24]. 


\subsubsection{Active Feedback Control}

Active feedback control systems attempt to either further reduce the effective impedance experienced by the beam [25] [26] [27] [28] [29] or to damp the unstable oscillations through the cavity voltage or a correcting longitudinal field. The LLRF loops employed show great similarities with the use of negative feedback around an operational amplifier to reduce its output impedance.

At PEP-II, there were two LLRF feedback loops around the RF station attempting to lower the effective impedance experienced by the beam as shown in Figure 3.9. The

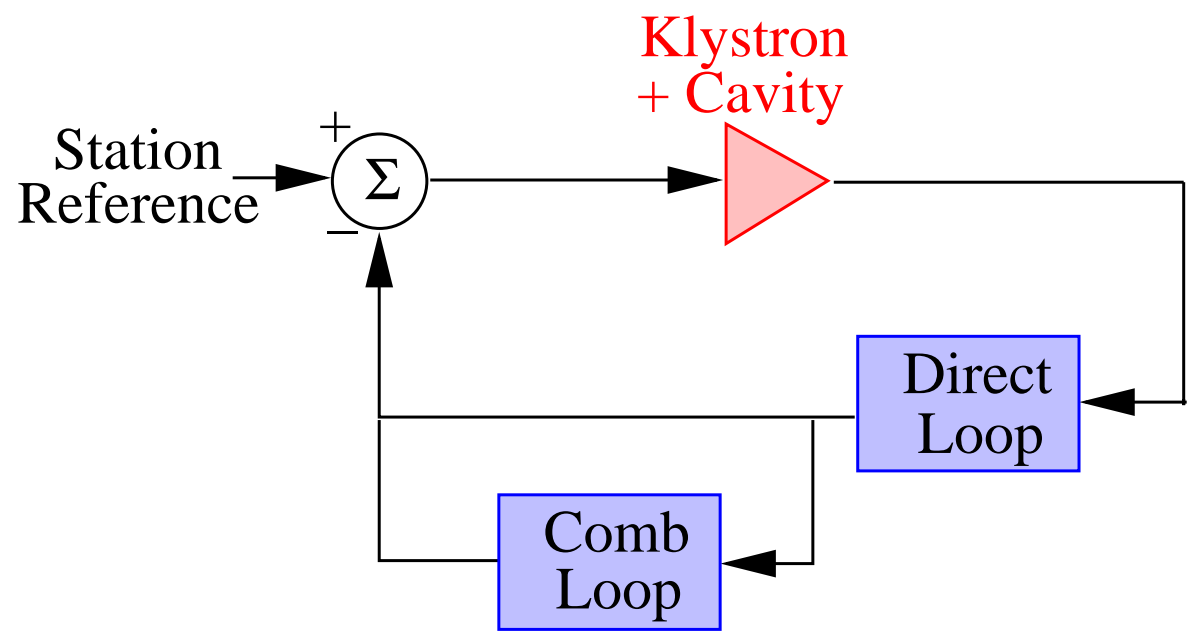

Figure 3.9: LLRF Feedback Loops.

Direct loop was an analog proportional feedback system with high loop gain, limited by the group delay. The Direct loop causes the station to follow the RF reference adding regulation to the total cavity voltage per station (2 or 4 cavities per station for PEP-II), thus extending the beam-loading Robinson stability limit and lowering the effective fundamental impedance seen by the beam. The Comb loop consists of a second order digital infinite impulse response (IIR) filter that adds narrow gain peaks at synchrotron sidebands around revolution harmonics - the frequencies where the beam "samples" the impedance - to further reduce the residual impedance. The combined effect of the Direct and Comb loops was an impedance reduction of almost two orders of magnitude at the synchrotron sidebands. It should be noted that these 
two feedback systems only reduce the beam instability contribution due to the cavity fundamental impedance. The stability of the RF system itself depends on the phase shift around the klystron/cavity path.

The configuration of these two loops were critical to achieve RF station stability and impedance control (beam stability). There were 5 adjustable parameters in the LLRF feedback loops: the direct loop gain $G_{d}$, direct loop phase shift $\phi_{d}$, comb loop gain $G_{c}$, comb loop delay $T_{c}$, and comb loop phase $\phi_{c}$. One of the goals of the simulation and modeling efforts presented in this work was to achieve a better understanding of the RF station-beam interaction and its effect on the dynamics of these two systems, as well as the sensitivity of the interaction on each of these 6 parameters.

Since the oscillation growth rates are proportional to the beam current (Equation 2.20), at operating currents the impedance reduction systems described above were not sufficient to achieve stability for modes near the cavity fundamental. A digital processing, narrowband feedback system was developed to control low mode instabilities, the Low Group Delay Woofer (LGDW) [30], [31]. The LGDW used the error signal received from Beam Position Monitors (BPMs) to compute a band limited correction signal for the RF station voltage reference. Data for the performance of the LGDW, as well as the Direct and Comb loops will be presented in Chapter 5 .

For the HOM modes, a damping system was necessary. A bunch-by-bunch system, the Longitudinal FeedBack (LFB), was developed for PEP-II, ALS, and DA $\Phi$ NE [32] [33] [34] [35] [36] [37] [38], and eventually also installed at SPEAR, BESSY-II, and PLS [39], [40]. In this system, the error signal received from the BPMs for each bunch is individually processed by a farm of Digital Signal Processing processors and the correction signal implemented by wide-band kickers. The error signal is common for the LFB and LGDW. Returning to Equation 2.8, the wide-band kicker attempts to apply a voltage $V_{n}^{k}(t)=-V_{n}^{w k}(t)$. The performance of the LFB system, was expected to be limited by delays, beam noise, DSP dynamic range, but mostly by the available amplifier power to the kickers (limited by cost). Operational conclusions on the LFB performance limitations have been presented in [41]. The LFB successfully damped the HOM driven motion up to the highest currents achieved during PEP-II 
operations. A more detailed description of the LFB will be presented in Appendix A.

\subsubsection{Active Feedback Implementation Example}

The impedance control and subsequent growth rate reduction achieved by the active feedback control systems are illustrated in the following example. In this example, the LHC technical implementation and LLRF characteristics have been used to generate realistic representations of the impedance and corresponding growth rates. Figures 3.10 and 3.11 represent the naked cavity impedance and growth rates. The

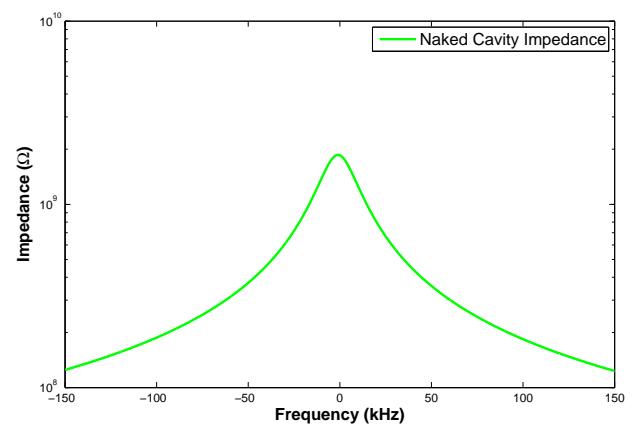

Figure 3.10: Naked cavity impedance.

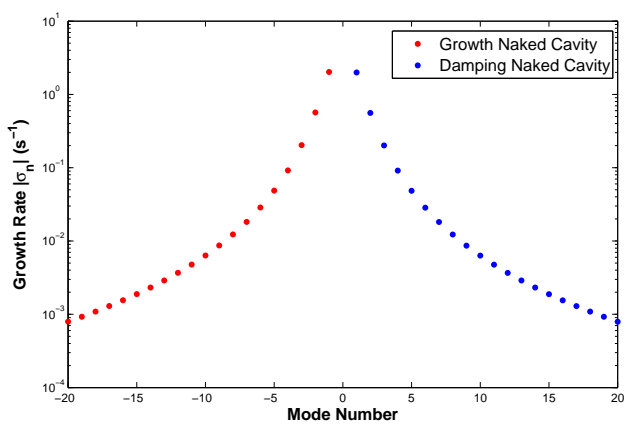

Figure 3.11: Naked cavity growth rates.

high Q resonance of the cavity is evident and results in very high growth rates. Points in red correspond to growing modes whereas points in blue are damped modes.

The Direct Loop is then employed to reduce the cavity impedance in closed loop. Figures 3.12 and 3.13 show the impedance and growth rate reduction due to the Direct Loop. The fastest growing rates are reduced by up to two orders of magnitude. On the other hand, the impedance smoothing increases the growth rates for modes further from the RF resonance. The effect of the Direct Loop is limited by the group delay; excessive feedback gains bring the loop to the onset of oscillation and instability.

The Comb Loop is introduced to deal with the reduced gain and phase margins and to further reduce the impedance. The phase of the Comb Loop wraps multiple times around the origin but the loop is stable. To achieve this the filter has high gain around the synchrotron sidebands, and low gain where the phase is $180^{\circ}$ to 


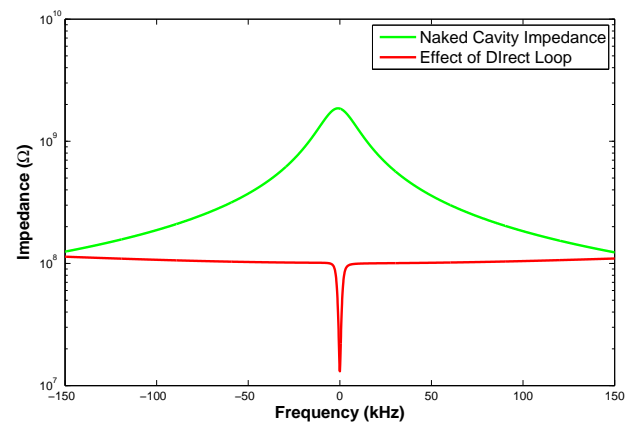

Figure 3.12: Impedance reduction due to the Direct Loop.

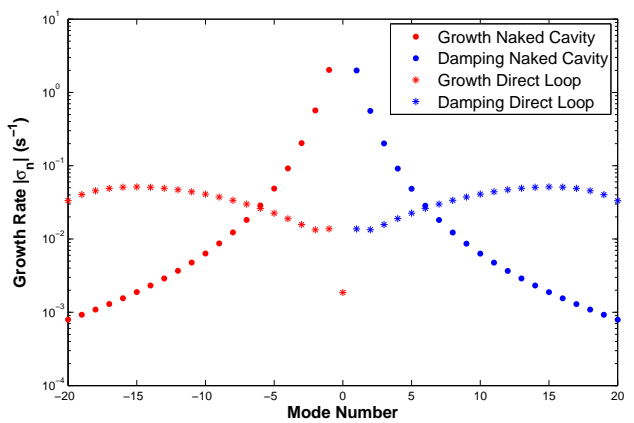

Figure 3.13: Growth rate reduction due to the Direct Loop.

ensure satisfactory gain and phase margins. The resulting reduction in impedance and growth rates is depicted in Figures 3.14 and 3.15.

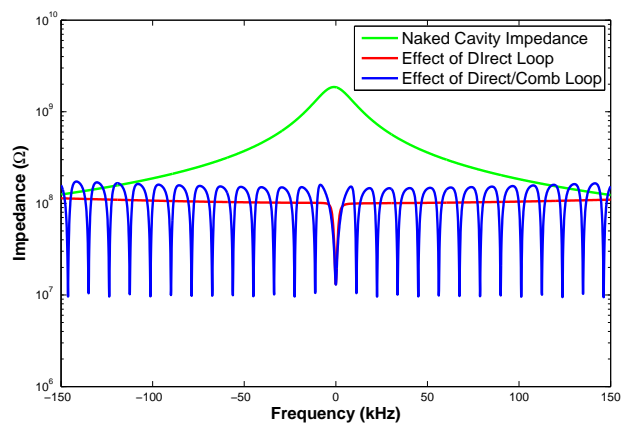

Figure 3.14: Impedance reduction due to the Comb and Direct Loop.

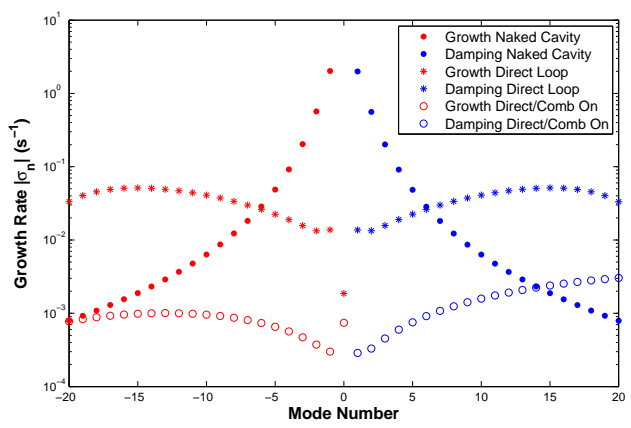

Figure 3.15: Growth rate reduction due to the Comb and Direct Loop. 


\section{Chapter 4}

\section{PEP-II Time-Domain Simulation: Motivation and Development}

As noted in Chapter 3, the theoretical study of the RF station-beam interaction is difficult due to the complexity of the multiple feedback loops and the non-linear nature of the system. Furthermore, the LLRF parameters have to be selected to maximize RF feedback loop and beam stability margins. The multiple RF stations and varying number of cavities per station further increase the complexity. An empirical approach for the study of the system and the selection of optimal parameters would not only require a lot of machine time and suggest risks for system components, but also would not allow for an arbitrary variation of system parameters. Therefore, a timedomain simulation was developed to study this interaction. Estimating the effect of RF configurations on beam dynamics, determining optimal settings, and studying alternative hardware designs, are an integral part of this work.

This Chapter focuses on the development of the time-domain simulation. Section 4.1 presents the motivation for and goals in developing the time-domain simulation. Section 4.2 describes the PEP-II longitudinal system whereas Section 4.3 outlines the simplifications, assumptions, and general structure of our model. The techniques for measuring system transfer functions and growth rates are shown in Sections 4.4 and 4.6 respectively. The time-domain simulation validation based on the transfer function and growth rates measurements is then described in Sections 4.5 and 4.7. 


\subsection{The Simulation's Motivation and Goals}

The motivation for this tool was to explore the stability margins and performance limits of PEP-II RF systems at future higher currents and upgraded RF configurations. It also served as a test bed for new control algorithms and defined the ultimate limits of the LLRF architecture. It also provided insight into subtle behaviors of the system and suggestions for optimal tuning (as with the comb rotation). The simulation model was very helpful in obtaining insight into the effect of the variations in the klystron responses and system imperfections. In the effort to study the RF station-beam dynamics interaction, the simulation's ability to separate the stability of the particle beam from that of the RF station was very helpful.

The simulation allowed us to make a complete study of the system sensitivity to LLRF parameters, determine the trade-offs between LLRF and beam stability, try alternative configuration algorithms, estimate the architecture's limits before they were reached, and propose alternative technologies. These accomplishments were achieved with minimum machine time used for system-simulation verification and helped break current records during the last PEP-II run.

\subsection{PEP-II Rings - Longitudinal Systems}

The PEP-II facility consists of two independent storage rings. The HER (9 GeV electron beam) and the LER (3.1 GeV positron beam) each have harmonic number $h=3492$ and operate at the RF frequency of $476 \mathrm{MHz}$. Continuous injection is achieved using collision energy electrons and positrons from the SLAC Linac and Damping Ring complex. The HER contains 11 RF stations, while the LER is composed of 4 RF stations. Each PEP-II station has a 1.2 MW klystron. The klystrons are built by SLAC, Phillips and Marconi and each design has different characteristics and performance. Some klystrons power 2 normal-conducting RF cavities whereas others power 4 normal-conducting RF cavities. The RF cavities have high-order mode dampers and an $R / Q$ ratio of 116 .

The LLRF systems include direct and comb loop feedback paths to reduce impedances 
seen by the beam as described in Section 3.2. The stations also incorporate numerous low bandwidth regulating loops which control the cavity tuners, the high-voltage power supply voltage and compensate for gap transient effects [42], [43]. The tuner loop detunes the cavity for minimum reflected power, whereas the klystron saturation loop maintains constant saturation headroom by controlling the high-voltage power supply to the klystron. The gap feedback loop removes revolution harmonics from the feedback error signal to avoid saturating the klystron. A block diagram of one of the RF stations is shown in Figure 4.1. A more detailed description of the feedback

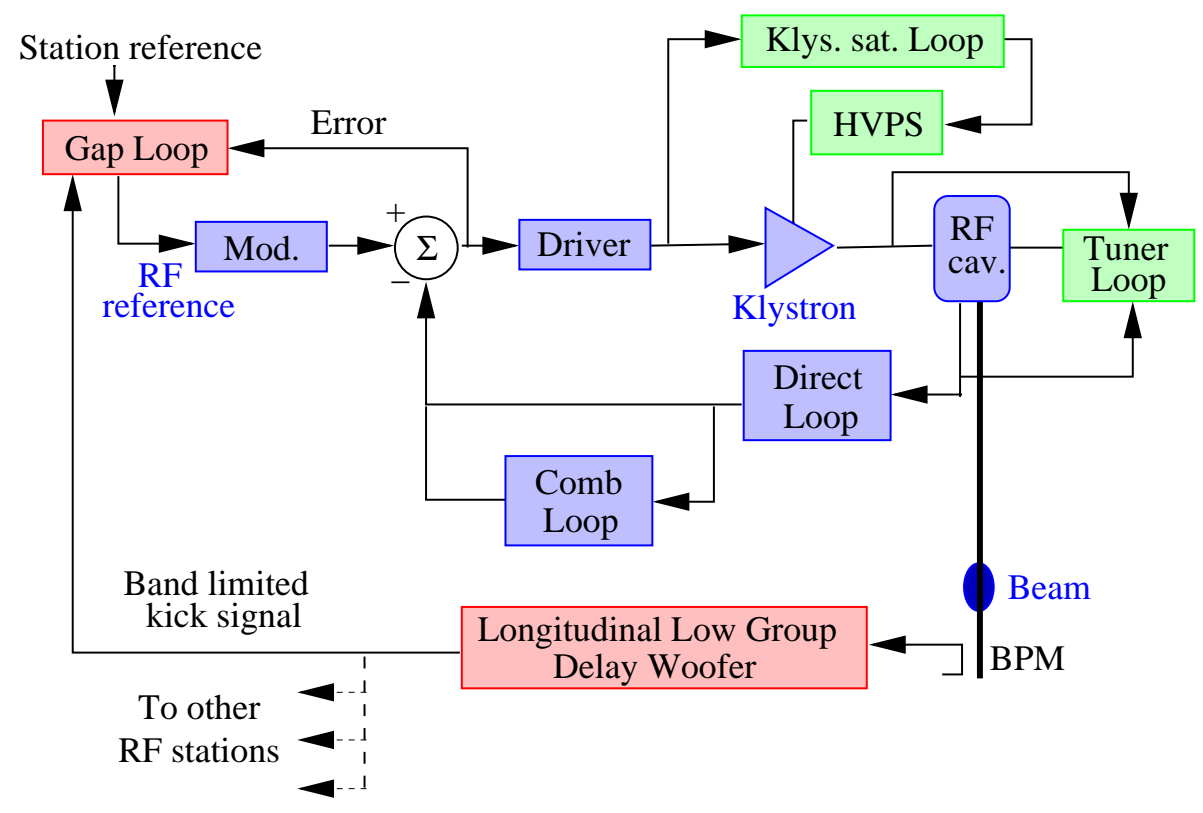

Figure 4.1: PEP-II RF station block diagram.

loops and their purpose, as well as of the numerous low bandwidth regulating loops can be found in [42].

In Section 2.2, we presented how the bunch motion is transformed into the modal basis given by Equation 2.15. For the PEP-II case (with an RF frequency of $476 \mathrm{MHz}$, a revolution frequency of $136 \mathrm{kHz}$, and a harmonic number of 3492 buckets), a bytwo filling pattern $(N=h / 2)$ yields 1746 filled bunches. The phase oscillations of these bunches can be decomposed into 1746 even-fill modes. Mode $n$ corresponds to a phase difference between adjacent bunches of $2 \pi(n / 1746)$, where $n$ ranges from 0 to 
1745 [44]. Therefore, modes repeat with a period of 1746 , so that modes 873 to 1745 are equivalent to modes -873 to -1 . Thus, all the modes can be represented with the range from -873 to 872 . This modal representation has been used in this work.

In the system block diagram in Figure 4.1, the blocks in green represent slow varying system components (in the order of a few $\mathrm{Hz}$ ), which are effectively constant for the time intervals related with coupled-bunch instabilities (a few ms) and therefore are not modeled in the simulation. The blocks in blue represent the RF station-beam interaction. As described in Section 3.2, even though the LLRF feedback achieves a significant reduction of the impedance experienced by the beam, the RF stationbeam system is unstable for operating currents. The LGDW is then employed to stabilize the system. The gain of the LGDW though is limited due to the group delay. Therefore, for each operational configuration the LLRF must be optimized to achieve the maximum possible impedance reduction, as will be described in Section 4.4.

\subsection{Model Description}

As described earlier, the simulation is focused on understanding the interaction among the low-order dynamics of the beam, the cavities and the fast LLRF feedback loops. This tool is developed as a block system in Simulink, which uses the system parameters calculated in Matlab to set the initial conditions of the slow loops and to provide measurement/estimation tools. The top level Simulink block system for PEP-II is shown in Figure 4.2. Some simulation elements were based on earlier work developed by R. Tighe [45]. The Matlab algorithms for the LHC implementation of the time-domain simulation have been published in [46].

Loops in green in Figure 4.1 are slow in nature and set the high-voltage power supply magnitude or the tuner position for the cavities. In the time frame in which the simulation characterizes the dynamic interaction between the beam and the RF stations, the changes in the variables controlled by the slow loops are negligible. These slow variables are set via parameters in the simulation, which can be calculated from the initial conditions to define the operation point of the system. Based on these described model simplifications, the simulation complexity is scaled to the minimum 


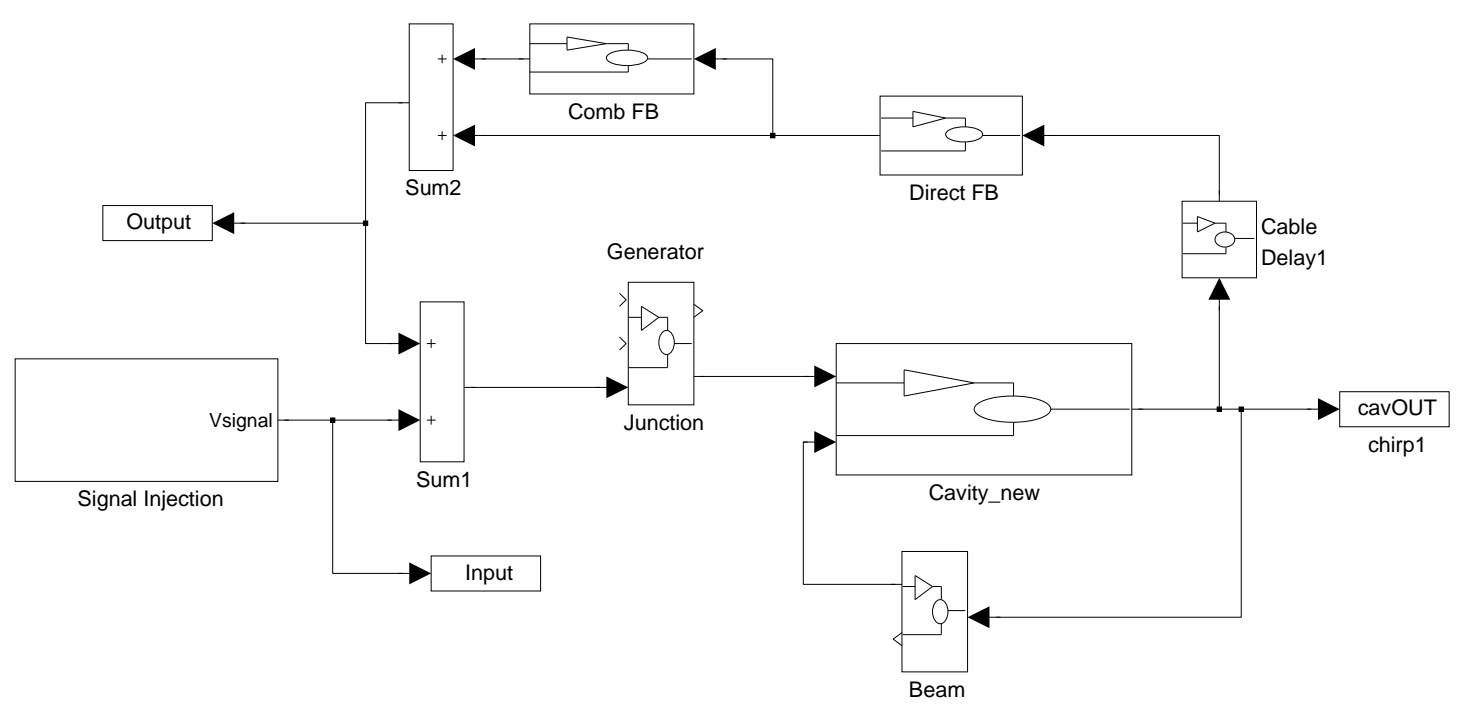

Figure 4.2: Simulink Block Diagram for PEP-II LER.

required to reproduce the essential physical dynamics. The system reduced model is depicted in the simplified block diagram in Figure 4.3. The earlier studies by R. Tighe attempted to determine the stability of the beam including the active longitudinal feedback. In this work, we determine the value of the dominant system eigenvalue achieved by the LLRF and then compare this value to the capture range of the LGDW as determined by separate measurements. This approach helps gain insight on the budgeting between the two systems and on the sensitivity of each on essential machine parameters.

As shown in Figure 4.3 the 2 cavity and 4 cavity stations were represented by different macrocavities, to account for their significantly different operating voltage and detuning. The LER model did not include the macrocavity representing the 4 cavity stations, since the ring only used 2 cavity stations.

The individual responses of the klystron driver amplifiers at PEP-II had a significant effect on the beam dynamics, as will be presented in Section 5.5. This discovery added to the complexity of the HER simulations due to the large number of stations and the variation among the amplifier responses. To include the individual amplifier responses without significantly increasing the computational load, the HER stations were simulated individually. Since the growth rates are proportional to the total 


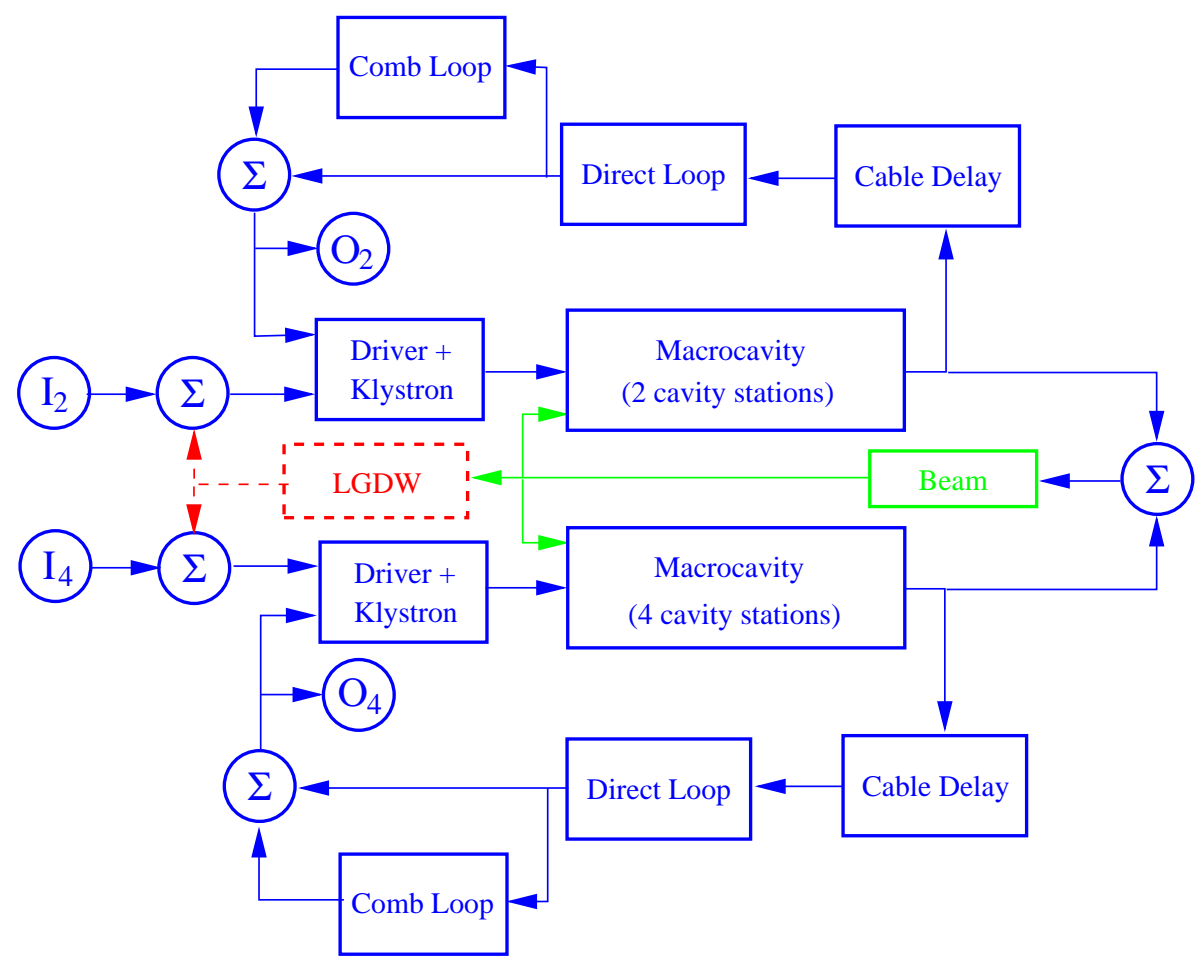

Figure 4.3: Simplified System Block Diagram. The system transfer function is measured between the Inputs $\left(I_{2}\right.$ and $\left.I_{4}\right)$ and Outputs $\left(O_{2}\right.$ and $\left.O_{4}\right)$. The macrocavity representing 4 cavity stations is only used for the HER model.

impedance presented to the beam (Equation 2.20), and the total impedance is the sum of the impedance of each station, it is possible to estimate the growth rates for each station individually and then sum over all stations. This treatment though excludes effects on the beam dynamics due to the interaction among the various RF stations. To represent this simplification in Figure 4.3 the signal from the 2 and 4 cavity stations is added before the application to the beam, contrary to the physical system where the interaction is sequential.

As depicted in Figures 4.1 and 4.3, the important blocks that affect the dynamic interaction between the beam and the RF station are the direct loop, the comb loop, the klystron, the cavity, and the group delays associated with the signal propagation around the station. Those elements are represented in the simulation as discrete blocks: 
- the klystron, including the saturation curve and the driver amplifier frequency response for each PEP-II RF station

- the cavity, with an RLC equivalent circuit model. Two different models are included for 2 cavity and 4 cavity stations (only for the HER)

- the LLRF system, using ideal models of the processing filters with lead/lag networks and including quantizing effects in the digital IIR. Individual electronic components are not included, but representative noise models are added as needed

- the beam, the total current is divided among "macrobunches" to preserve low order modes. The phase deviation $\phi$ for the bunch centroid is monitored. The phase information connects the simulation with the theoretical background developed in Chapter 2 and is processed to provide the modal growth rates

- cable, processing delays are included.

These elements are described in more detail below.

The overall dynamic system is of complex structure, including a large number of state variables with different dynamics that makes simulating at this level cumbersome. The longitudinal dynamics of the 1746 individual bunches can be modeled, based on energy considerations, by Equation 2.13. The goal of the simulation is to study the low-order mode behavior of the beam induced by the interaction with the RF stations. Thus, the particle beam is modeled via a variable number of macrobunches $N=72$ comparable to the IIR comb filter samples per turn, rather than the 1746 physical bunches. From Equation 2.15 we can see that modes -35 to 36 can be fully resolved with 72 macrobunches. Since modes -10 to 10 cover the full bandwidth of the RF station, the macroparticle reduction still allows us to fully resolve the low-order beam modes and interaction with the RF fundamental impedance. Furthermore, this approach reduces the number of state variables assigned to model the beam dynamics, but allows keeping the same abort gap in the filling pattern. An ion clearing gap of approximately $5 \%$ of the train was employed at PEP-II. 
The energy $q V\left(\tau_{n}+\tau_{s}\right)$ applied per turn to the $n^{\text {th }}$ bunch is the net contribution of all the RF cavities in the ring. The voltage $V$ can be expressed by

$$
V\left(\tau_{n}+\tau_{s}\right)=\sum_{i=1}^{S T} \sum_{j=1}^{K} V_{i, j}\left(\tau_{n}+\tau_{s_{i, j}}\right)
$$

where $S T$ is the number of stations, $K$ is the number of cavities per stations $(K=2$ in the LER and $K=2$ or $K=4$ in the HER), and $v_{i, j}$ is the instantaneous voltage corresponding to the $j^{\text {th }}$ cavity in the $i^{\text {th }} \mathrm{RF}$ station. In nominal operation, the cavities per station are detuned by the same magnitude which allows us to group either the two or the four cavity station in a unique dynamic macromodel (a 2 or 4 cavity macromodel). This simplification defines the voltage per station as

$$
\begin{aligned}
V_{s_{i}}(t) & =\sum_{j=1}^{K} V_{i, j}(t) \quad \text { with } K=2 \text { or } 4 \\
& =2 V_{i}(t) \text { or }=4 V_{i}(t) .
\end{aligned}
$$

Further simplification in the simulation is possible by considering that in normal operation the voltages of all the stations present almost the same relative phase with respect to the beam. In that case, (4.1) can be simplified to

$$
V\left(\tau_{n}+\tau_{s}\right)=\sum_{i=1}^{S T} V_{s_{i}}\left(\tau_{n}+\tau_{s_{i}}\right) \approx \sum_{i=1}^{S T} V_{s_{i}}\left(\tau_{n}+\tau_{s}\right)
$$

These simplifications represent the cavities for all the ring RF stations in two macromodels (macrocavities). All the 4 cavity RF stations interactions are lumped into a single 4 cavity macrostation and all the 2 cavity RF stations are similarly modeled via a single 2 cavity macrostation. The reason behind the development of two separate macrocavities are the differences in operation point of a two-cavity RF station and a four-cavity RF station. For example, the tuned resonance frequency of the cavities can be significantly different in each case.

The simulation models the RF signals in baseband and uses the in-phase/quadrature 
formalism to represent them. Macrocavities modeled under this formalism are represented by a reduced model defined by

$$
\frac{d V_{m}(t)}{d t}=A\left(\omega_{r}\right) V_{m}(t)+B I_{k l y}\left(t-t_{w}, V_{D C}\right)+B I_{\text {beam }}(t),
$$

where $[A, B]$ is the state representation of the cavity, $V_{m}(t)=\left[\begin{array}{ll}V_{m_{I N}} & V_{m_{Q}}\end{array}\right]^{T}$ is the in-phase/quadrature macrocavity voltage vector, $\omega_{r}$ is the resonance frequency of the cavity, $t_{w}$ is the delay of the wave-guide between the klystron and the cavities, $V_{D C}$ is the station high-voltage bias and $I_{k l y}\left(t, V_{D C}\right), I_{\text {beam }}(t)$ are the in-phase/quadrature klystron and beam current vectors, respectively.

The simulation includes the effective impedance presented by all the stations to the beam, representing the collective effect of all the cavities and their feedback loops through the combination of 2 and/or 4 macrocavity stations. The effect of the accelerating fundamental RF impedance on the coupled-bunch instability has been presented in Section 2.3. In particular, Equation 2.20 quantifies the dynamic interaction between the beam and the fundamental longitudinal impedance around the operation point. Equation 2.20 defines the eigenvalues of the beam dynamics in the beam modal frame. The effect of the longitudinal effective impedance is evident on the modal damping and the deviation of the synchrotron frequency of the individual modes with respect to $\omega_{s}$.

The longitudinal effective impedance is determined by the RF cavity impedance and the action of the fast feedback loops at each RF station. The RF cavity impedance per station is modified by the feedback loops as

$$
Z_{i}(\omega)=(I+G(\omega) H(\omega))^{-1} Z_{s t_{i}}(\omega),
$$

where $G(\omega) H(\omega)$ corresponds to the return ratio of the station and $Z_{s t_{i}}(\omega)$ is the frequency response of the RF cavities. Adjustable parameters in the control loops and the stations define the frequency response of the system and the stability of the RF feedback loops. The overall station impedance $Z_{i}\left(l \omega_{0}+\omega_{s}\right)$ at frequencies $l \omega_{0}+\omega_{s}$ corresponds to the beam perturbation. This impedance is minimized by optimizing the LLRF station parameters, compatible with stability performance criteria for the RF 
loops. It is important to recognize that the overall stability of the system is comprised not only of the stability of the LLRF control loops, but also of the beam stability affected by the interaction with the longitudinal impedance of the RF stations.

\subsection{Identification and Configuration Algorithms}

From the discussion on the beam dynamics-RF interaction, it is obvious that a method is necessary to not only characterize, but also configure the LLRF system to minimize the effective impedance experienced by the beam, satisfying at the same time gain and phase margins for the RF stations. The LLRF is configured using a model of the RF station. The parameters of the model for a given operation point are determined from a measured closed loop transfer function, as described in detail below.

In PEP-II operations, station parameters are configured using a non-invasive method developed by D. Teytelman that starts with the identification of the closedloop transfer function of each station [47]. The motivation behind this method is the inability to measure the open-loop transfer function of the station with beam in the machine, because opening the LLRF control loops causes loss of the impedance control. While it is possible to measure the open-loop RF station transfer function at zero current, and hence study the closed-loop stability margins, as the machine is filled the RF station dynamics change significantly, since many station parameters vary with operation point. To best configure the LLRF parameters at operating currents, the closed-loop system transfer function is first measured injecting a complex time domain excitation at the input, as marked in Figure 4.3. The time domain response of the station is sampled at the output and recorded. The closed-loop transfer function $H_{\text {meas }}(\omega)$ is estimated using the correlation method based on the measured input/output records.

Equation 4.3 is presented in the in-phase/quadrature (IQ) formalism, where $H(\omega)$ is a 2 -by-2 matrix. The measured transfer function $H_{\text {meas }}(\omega)$ is a complex quantity 
though. Their correspondence is obvious from this simple example:

$$
\begin{aligned}
\vec{V}_{\text {meas }}(\omega) & =M \vec{V}_{\mathrm{inj}}(\omega) \\
{\left[\begin{array}{c}
V_{I_{\text {meas }}}(\omega) \\
V_{Q_{\text {meas }}}(\omega)
\end{array}\right] } & =\left[\begin{array}{cc}
\alpha & -\beta \\
\beta & \alpha
\end{array}\right]\left[\begin{array}{c}
V_{I_{\text {inj }}}(\omega) \\
V_{Q_{\text {inj }}}(\omega)
\end{array}\right]=\left[\begin{array}{c}
\alpha V_{I_{\text {inj }}}(\omega)-\beta V_{Q_{\mathrm{inj}}}(\omega) \\
\beta V_{I_{\text {inj }}}(\omega)+\alpha V_{Q_{\text {inj }}}(\omega)
\end{array}\right] \\
V(\omega) & =V_{I_{\text {meas }}}(\omega)+i V_{Q_{\text {meas }}}(\omega) \\
& =\left(\alpha V_{I_{\text {inj }}}(\omega)-\beta V_{Q_{\text {inj }}}(\omega)\right)+j\left(\beta V_{I_{\text {inj }}}(\omega)+\alpha V_{Q_{\text {inj }}}(\omega)\right) \\
& =(\alpha+j \beta) V_{I_{\text {inj }}}(\omega)+j(\alpha+j \beta) V_{Q_{\text {inj }}}(\omega) \\
& =(\alpha+j \beta)\left(V_{I_{\text {inj }}}(\omega)+j V_{Q_{\text {inj }}}(\omega)\right)
\end{aligned}
$$

where $H_{\text {meas }}(\omega)=\alpha+j \beta$. Therefore, the matrix $M$ in the IQ formalism, corresponds to $H_{\text {meas }}(\omega)$ in the complex formalism. The structure of matrix $M$ assumes that the model is linear.

To obtain a parametric model of the closed-loop transfer function, the transfer function of a linearized model of the station, $H_{\text {model }}(\omega)$, is fitted to the estimated function $H_{\text {meas }}(\omega)$ for the given operation point by adjusting characteristic parameters in $H_{\text {model }}(\omega)$. The linearized model is parametrized by only 8 unknown parameters: cavity detuning frequency $\omega_{r}$, cavity loaded quality factor $Q_{l}$, direct loop gain $G_{d}$, direct loop delay $T_{d}$, direct loop phase shift $\phi_{d}$, comb loop gain $G_{c}$, comb loop delay $T_{c}$, and comb loop phase $\phi_{c}$. The model is characterized by only those parameters because the frequency responses of the lead-lag compensation, comb filter and the equalizer's finite impulse response (FIR) filter can be accurately modeled from the known hardware implementation. An estimated system response is then derived via least-squares fitting from the model parameters. In this process, some parameters are not adjusted by the fitting routine: $\omega_{r}$ is determined by the average cavity detuning as measured by the tuner position read-back and $Q_{l}$ is set to the nominal value based on the design cavity $Q$ and coupling factor $\beta_{0}$. The fitting routine is then based on a six-dimensional optimization including a frequency-weighted error function given by

$$
f(\vec{x})=\sum_{\omega} W(\omega)\left|H_{\text {meas }}(j \omega)-H_{\text {model }}(\vec{x}, j \omega)\right|^{2}
$$


where $\vec{x}$ is a vector of 6 optimization parameters and $W(\omega)$ is a weighting function. This is performed for a bandwidth of $1.5 \mathrm{MHz}$, yielding the 8 parameters that define the linear model. The linearized model for the station $H_{\text {model }}(\vec{x}, j \omega)$ in Laplace domain is expressed by

$$
H_{\text {model }}(s)=\frac{L_{\mathrm{dir}}(s)}{1-\left(1+L_{\mathrm{comb}}(s)\right) L_{\mathrm{dir}}(s)}
$$

where $L_{\mathrm{dir}}(s)$ is the open-loop transfer function of the direct loop and $L_{\text {comb }}(s)$ is the open-loop transfer function of the comb loop. These are given by

$$
\begin{aligned}
L_{\mathrm{dir}}(s) & =\frac{2 \sigma s}{s^{2}+2 \sigma s+\omega_{r}^{2}} H_{\mathrm{L}-\mathrm{L}}(s) G_{d} e^{j \phi_{d}-\left(s-j \omega_{\mathrm{rf}}\right) T_{d}} \\
L_{\mathrm{comb}}(s) & =H_{\mathrm{comb}}(s) H_{\mathrm{eq}}(s) G_{c} e^{j \phi_{c}-\left(s-j \omega_{\mathrm{rf}}\right) T_{c}}
\end{aligned}
$$

where $\sigma=\omega_{\mathrm{rf}} / 2 Q_{l}$ is the damping time of the cavity, $H_{\mathrm{L}-\mathrm{L}}(s)$ is the transfer function of the lead-lag compensation, $H_{\text {comb }}(s)$ is the transfer function of the comb filter and $H_{\text {eq }}(s)$ is the transfer function of the equalizer FIR filter.

This parameterization of the transfer function with quantities of physical meaning, allows the calculation of the open-loop transfer function of the station around the actual operation point and also helps insure a unique representation of the system. With the open-loop estimate, optimal values are calculated for both the direct loop gain and phase rotation parameters $\left(G_{d}, \phi_{d}\right)$ and the comb filter gain and phase rotation $\left(G_{c}, \phi_{c}\right)$. These optimal values are defined through the specification of open-loop gain and phase margins. Through this model-based technique, the LLRF systems of the physical machine are configured and studied over the range of highcurrent operating points, and the RF systems are periodically adjusted and "tuned" in operation.

It should be noted that these transfer function measurements are only possible if the system is stable, as is the case during operations. Noise is present in the system and degrades the estimation. During the development of the optimization and configurations tools empirical knowledge was used to maximize the signal to noise ratio by adjusting the injected signal level.

An example of the configuration of a PEP-II station is shown in Figure 4.4. The 

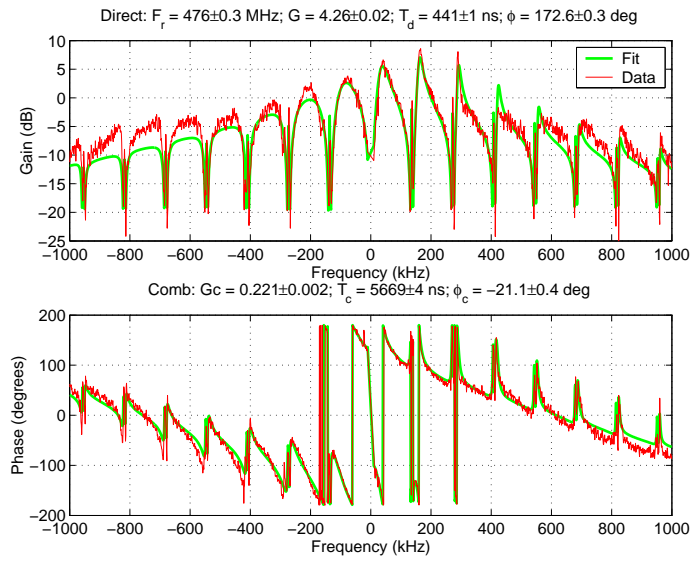

$\Downarrow$
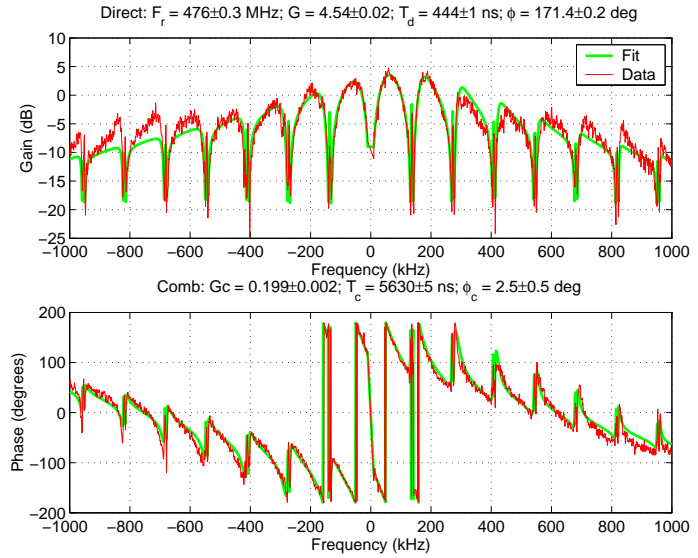

$\Downarrow$
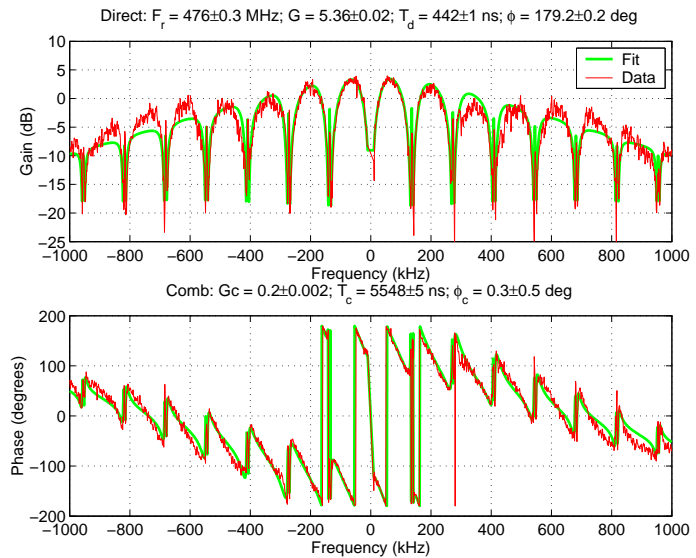

Figure 4.4: Successive optimization of RF station transfer function. The first transfer function demonstrates peaking, which is reduced in two steps by appropriate adjustments of the LLRF gain and phase. 
first transfer function demonstrates peaking, which corresponds to reduced gain and phase margins at those frequencies. As a consequence the RF station is on the onset of instability. As can be seen by the RF parameters fitted by the linear model, the comb gain is reduced from 0.22 to 0.2 in two steps, where as the direct gain is increased from 4.26 to 5.36. This figure shows the effectiveness of the optimization tools in improving the poor gain and phase margins.

A similar method is used in the time domain simulation to specify the parameters of the macrostation. To achieve agreement between the simulation and the physical system in the estimation of impedances and growth rates, it is important that the simulation defines an effective impedance interacting with the beam equal to the physical impedance presented by the RF stations to the beam. From 2.20 and (4.3), it is important to observe that this is possible only if there is agreement between the transfer function measured per station and the transfer function defined in the simulation. Consequently there should be close agreement between the linear model parameters fit to the physical station and the linear model parameters fit to the time domain simulation data.

\subsection{Transfer Function Validation in PEP-II}

Since the transfer function relationship between model and physical system implies a growth rate consistency, it is reasonable to use the transfer functions for an initial verification of the simulation model. Growth rate measurements were later used for a more careful and detailed test of the agreement between the real system and the simulation.

In Figures 4.5 and 4.6 the collected data are shown in red for measured (physical station) and simulated (model) transfer functions, respectively. Also shown in green are the fitted 6 parameter linear model responses $H_{\text {model }}(\omega)$ defined above.

From these figures we can clearly see the agreement between the data and the fit, which demonstrates the accuracy of the fitting tools. An important feature of the fitted linear model is its ability to compare the resulting sets of parameters extracted from the physical system and simulation. Their close agreement provides evidence of 


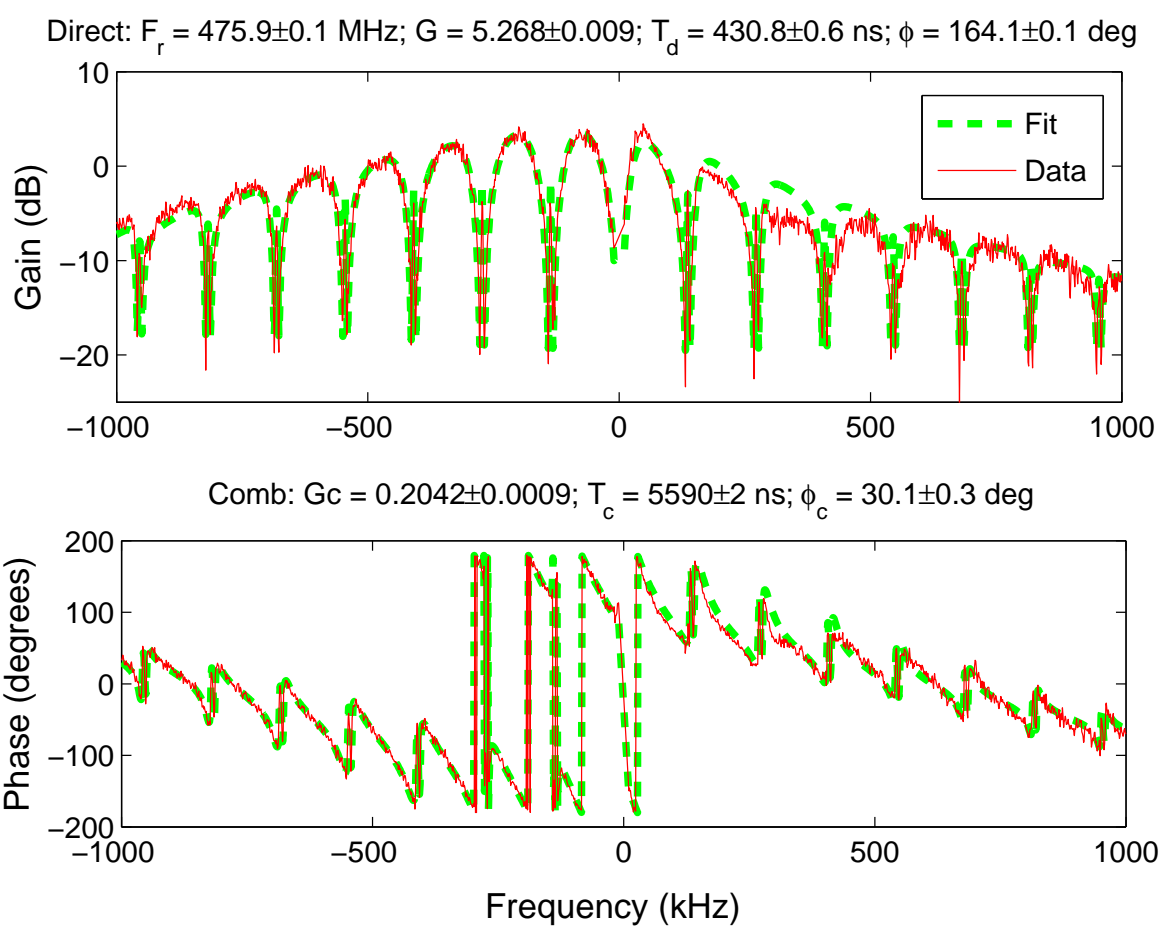

Figure 4.5: Transfer Function and parameters of operating station in LER at 1400 $m A$.

convergence of the simulation with the physical system. The measured and simulated transfer functions as well as the fitted parameters were very close providing confidence that the growth rates would also be comparable, as was later shown.

These transfer functions, while similar, are not identical. It is apparent from the studies of the multiple RF stations via these transfer functions and models that there are subtle but important variations in the physical RF stations. So the macro-model scheme must represent some sort of weighted average of all the stations to accurately model the beam dynamics. However, this macromodel would not fully capture the limits and dynamics of the LLRF stations themselves, or predict the limits of a particular physical station. The efforts to better understand the small discrepancies between the macromodel and the individual klystron physical transfer functions led to important further measurements of the physical stations which are analyzed later in Section 5.5. The discrepancy around $300 \mathrm{kHz}$ has been attributed to imperfections 

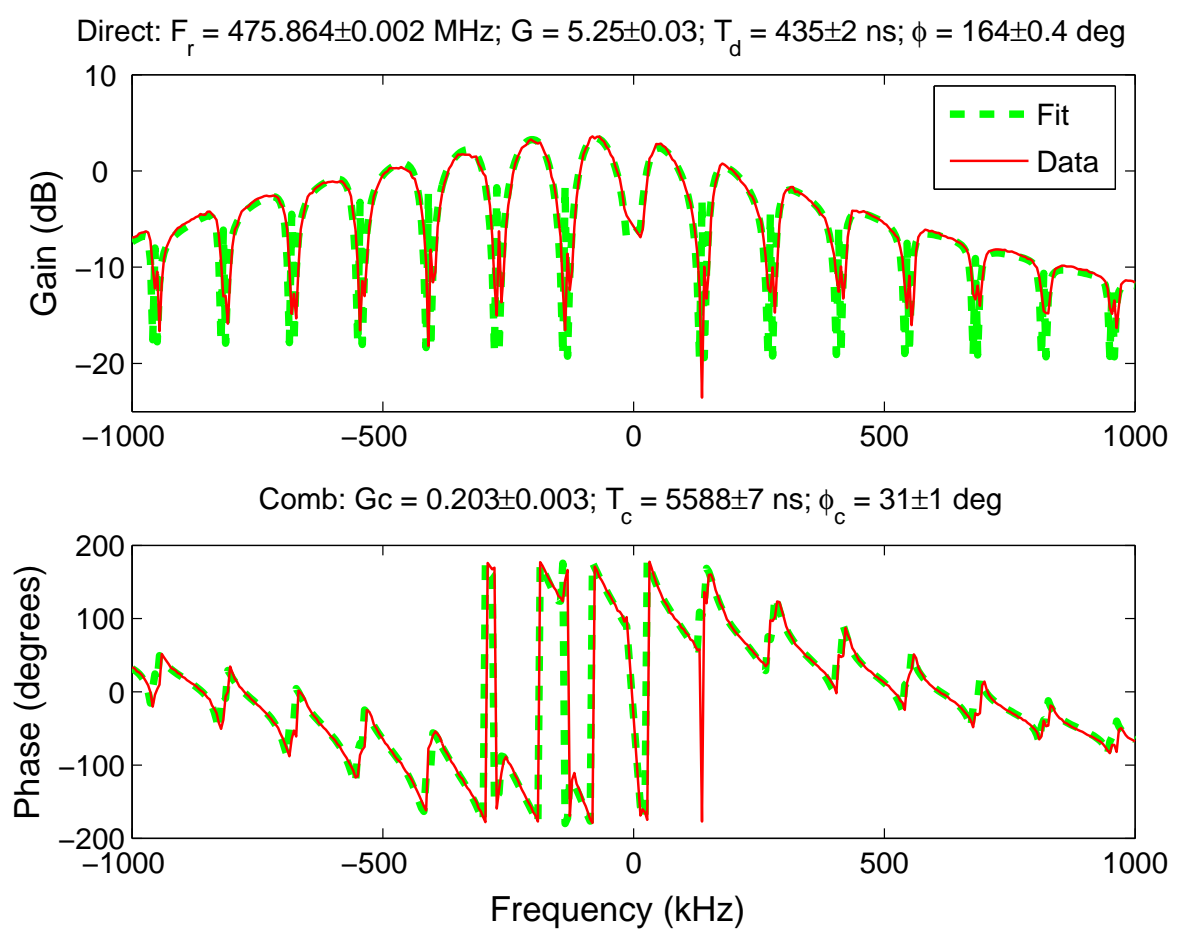

Figure 4.6: Transfer Function and parameters of macrostation from non-linear simulation in the LER at $1400 \mathrm{~mA}$.

of the radio frequency processor module (RFP), which leads to cross-coupling in the boards.

\subsection{Growth Rate Measurement Techniques}

The essential beam dynamic measurements from the simulation are the modal growth rates since these are used to quantify beam instability. The technique for measuring the growth/damping rates in PEP-II operations were first presented by S. Prabhakar [10] and then refined by D. Teytelman [4]. Using a related technique, naturally stable modes were studied by injecting a narrowband excitation to drive specific beam modes for a short period of time and observing the resulting natural decay transients. In PEP-II operations, the performance of the damping system in operation is evaluated by opening the longitudinal feedback, letting the unstable beam modes grow for a 
few milliseconds and then closing that feedback to damp the instability out. In this growth/damp technique, the transient process where the longitudinal loop is open should last a few milliseconds such that the unstable bunch amplitude does not escape the basin of attraction of the stable equilibrium point. Via transient-domain measurements of the bunches during both steps on the process, it is possible to measure the free growth rate of unstable modes and the overall damping performance of the closed-loop system.

It should be noted that while the damping loop is open, the operation point could potentially belong to a chaotic set. But, the experimental data from the growing motion during the few milliseconds in open loop follow an exponential growth rather than a random motion evident of chaos. In closed loop the system is locally stable.

The grow/damp analysis is performed in the beam modal domain transforming the measured beam phase into the even-filled modal base defined by Equation 2.15. The system dynamics when the longitudinal loop is open is characterized by Equation 2.19, where the growth/damping rate for the $l^{\text {th }}$ beam mode is defined by $\sigma_{l}$ in Equation 2.20. When the longitudinal feedback loop is closed the growth/damping rate for the $l^{\text {th }}$ beam mode can be defined by

$$
d_{l}=-d_{r}+\frac{\alpha e I_{0} \omega_{\mathrm{rf}}}{2 E_{o} T_{o} \omega_{s}} \mathcal{R}\left(Z^{\| \mathrm{eff}}\left(l \omega_{0}+\omega_{s}\right)-Z^{\| \mathrm{eff}}(0)\right)+\Delta_{l},
$$

where $\Delta_{l}$ is the effective feedback damping rate due to the longitudinal feedback. This technique allows measuring both $\sigma_{l}$ of the unstable beam modes and $d_{l}$ for the same modes, by examining the data during the growing phase when the loops are open for a few milliseconds and the capturing phase after the loops are closed again. The first reveals the interaction between the beam and the longitudinal impedance, while the second measures the net damping of the system, quantifying the performance of the longitudinal feedback loop.

To characterize the modal growth rates in the time domain simulation, a procedure similar to the first part of the growth/damp measurement technique is used. From an initial position of the beam near the equilibria, we let the beam naturally evolve in time and study the interaction between the RF station and the beam [30]. The 
advantage in the simulation is that due to the absence of the instrumental noise floor and the ability to start with appropriate initial beam conditions, the stable and unstable modes can be estimated concurrently. An example of the time-domain data is shown in Figure 4.7. The envelope of the synchrotron oscillations is growing as

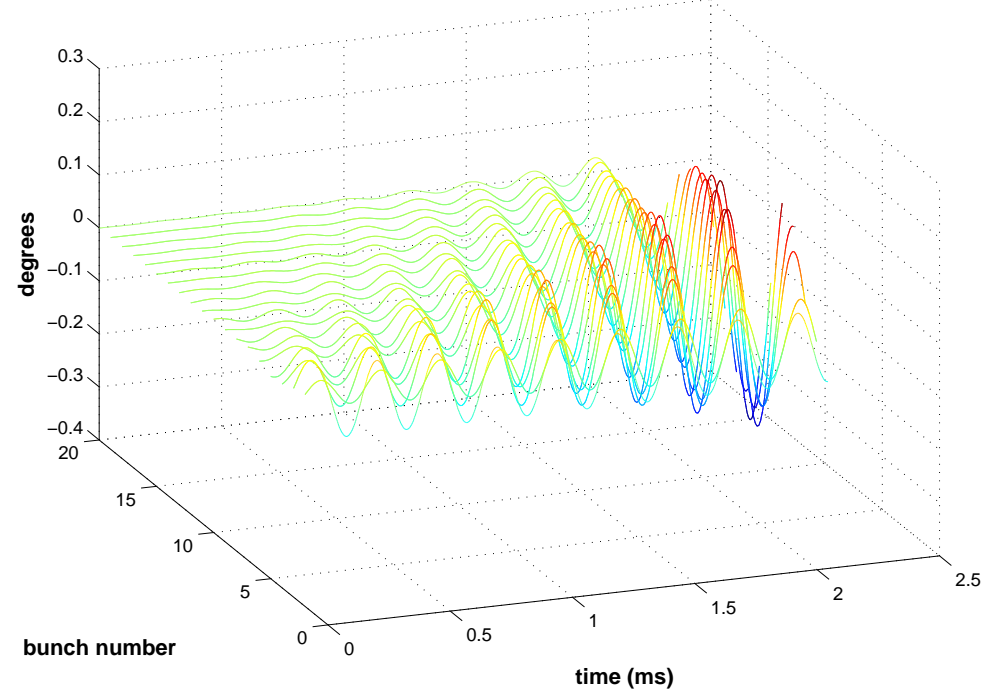

Figure 4.7: Time-domain data showing the phase deviation for 20 bunches over 2.5 ms (about 340 turns). The amplitude of the synchrotron oscillation is growing for all the bunches.

expected.

The time-domain data is then transformed to the modal domain. By fitting the time evolution of each mode, the natural complex frequency $\Lambda_{l}$ can be estimated following Equation 2.20. The real part of $\Lambda_{l}$ corresponds to the growth/damping rate and the imaginary part to the oscillation frequency of mode $l$. An example of the modal domain fit is shown in Figure 4.8, with the resulting growth rates for stable and unstable modes. To achieve consistency between the physical system and the simulation, the same growth rate extraction tools are used to analyze the time domain data in both cases. The simulated growth rates for modes -10 to 10 and their oscillation frequencies can be seen on the right in Figure 4.8 for the LER running at $2500 m A$. Modes -3 and -4 are usually the most unstable modes; the shift in mode 

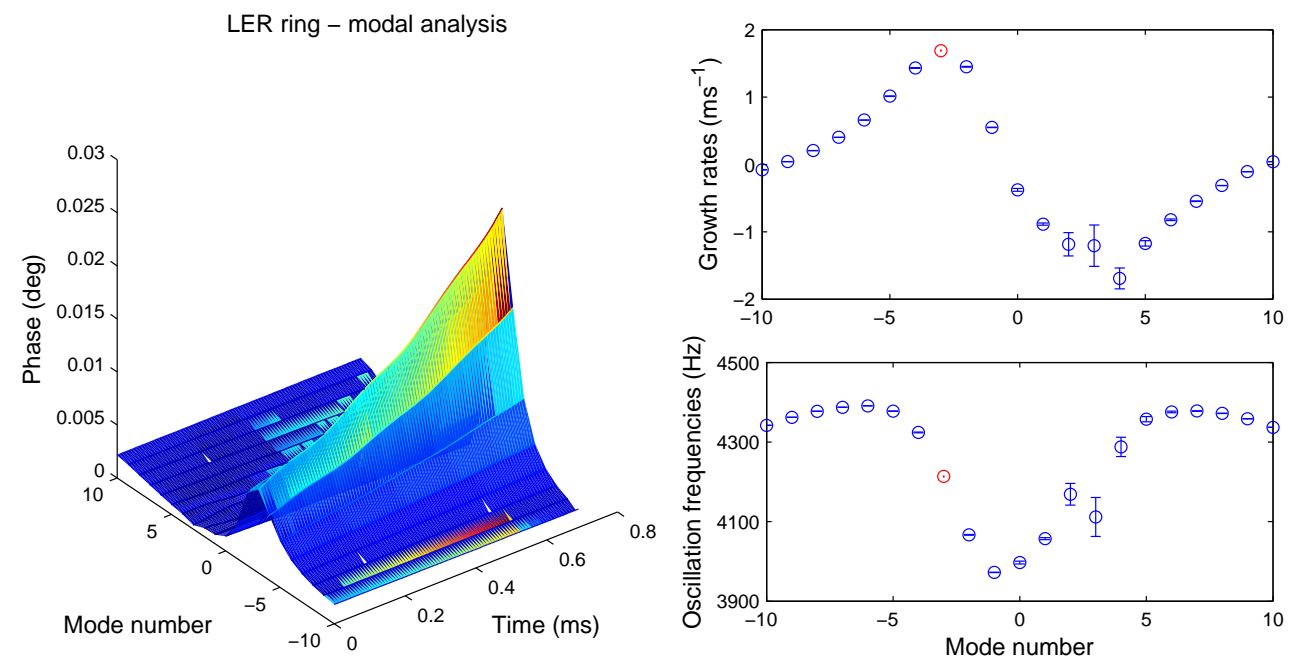

Figure 4.8: Simulated Low Frequency Beam Modes (LER at $2500 \mathrm{~mA}$ ).

number results from the change in cavity detuning with increasing beam current.

One application of this simulation is that the free growth rates can then be compared to the expected effective feedback damping rate $\Delta_{l}$ from the longitudinal loop, providing a quantitative measure of stability margins for each mode (in contrast to earlier work [45]). The effective feedback damping rate $\Delta_{l}$ is used as a metric because it is in the first order proportional to the beam current, until other effects become dominant at higher currents. The growth and damping rates $\sigma_{l}$ and $d_{l}$ on the contrary have some non-linear dependence on the beam current. In Figure 4.9 we can see an extrapolated line for the effective feedback damping rate with current based on three sets of measurements from the physical machine, as well as the estimated maximum achievable rate. The estimate for the maximum effective feedback damping rate is -6 to $-8 \mathrm{~ms}^{-1}$. The difficulty in estimating the limit is related to the fact that it cannot be directly measured in the physical system and that it changes with the system architecture. In this work the more conservative group-delay limit value of $-6 \mathrm{~ms}^{-1}$ will be used for estimations and predictions. 


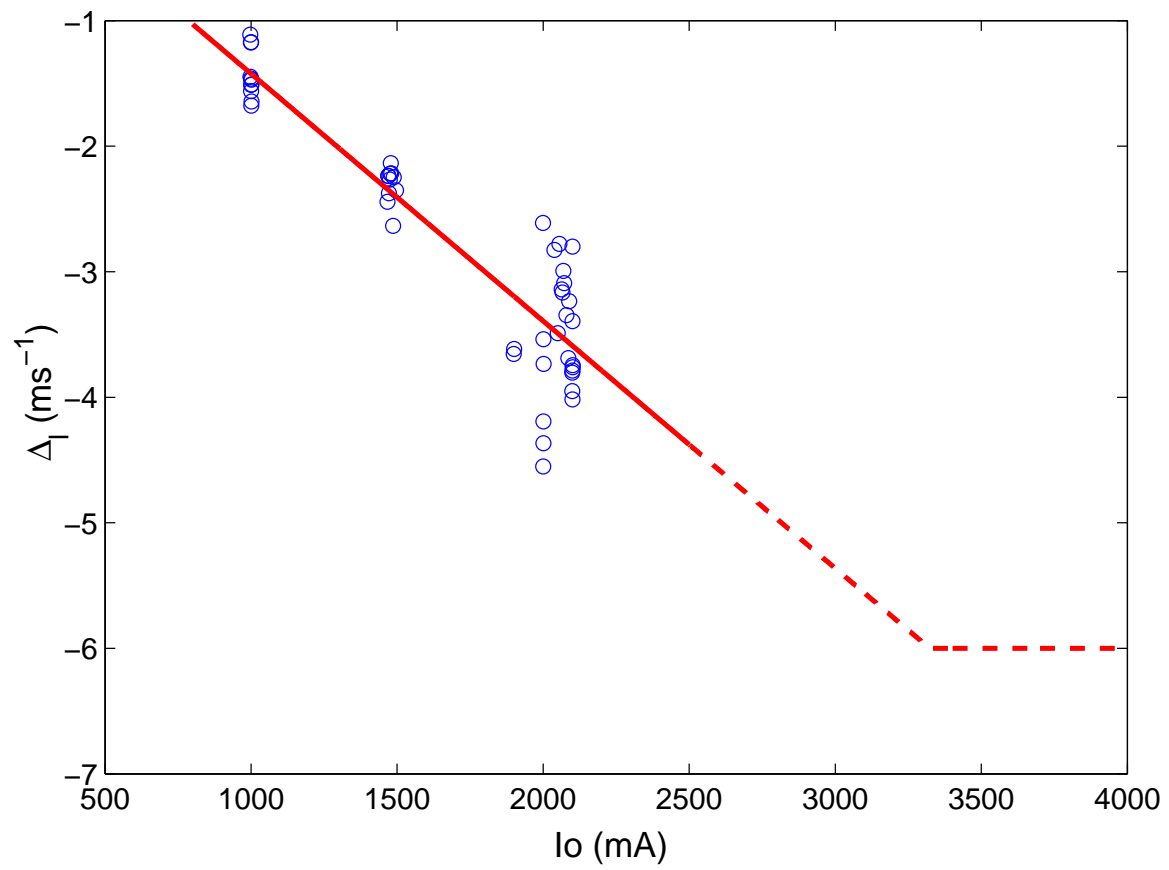

Figure 4.9: Plot of $\Delta_{l}$ with current in $m A$. The $-6 m s^{-1}$ limit is from the low-mode longitudinal control path.

\subsection{Growth Rate Validation}

To compare the results from growth/damp measurements performed in the LER at different currents and the simulation, RF stations at the LER and macrostations in the simulation were set with similar parameters and the growth rates were studied. In this case, results correspond to the LER operating with 4 RF stations each running at $1.25 \mathrm{MV}$, and with beam currents from 1400 to $2500 \mathrm{~mA}$. In these measurements the loop parameters of the RF stations were not set to the optimum values due to imperfections in the klystron driver and LLRF controllers (Section 5.5). The same linear model is fitted to both the real station and the simulation. In the simulation, several cases were analyzed to set the real klystron non-linear static transfer function per station. In each of these cases, the klystron non-linearity and the frequency response of the driver amplifier are included in the macrostation model, and the parameters of the closed loop are set so that the linear model fit to the macrostation 
is equal to that of the corresponding physical station. Under these conditions, the growth rates are measured in the simulation. This process is repeated in order to evaluate the interaction with the beam dynamics of each individual station in the LER.

Results of this validation are depicted in Figure 4.10, where multiple growth rates

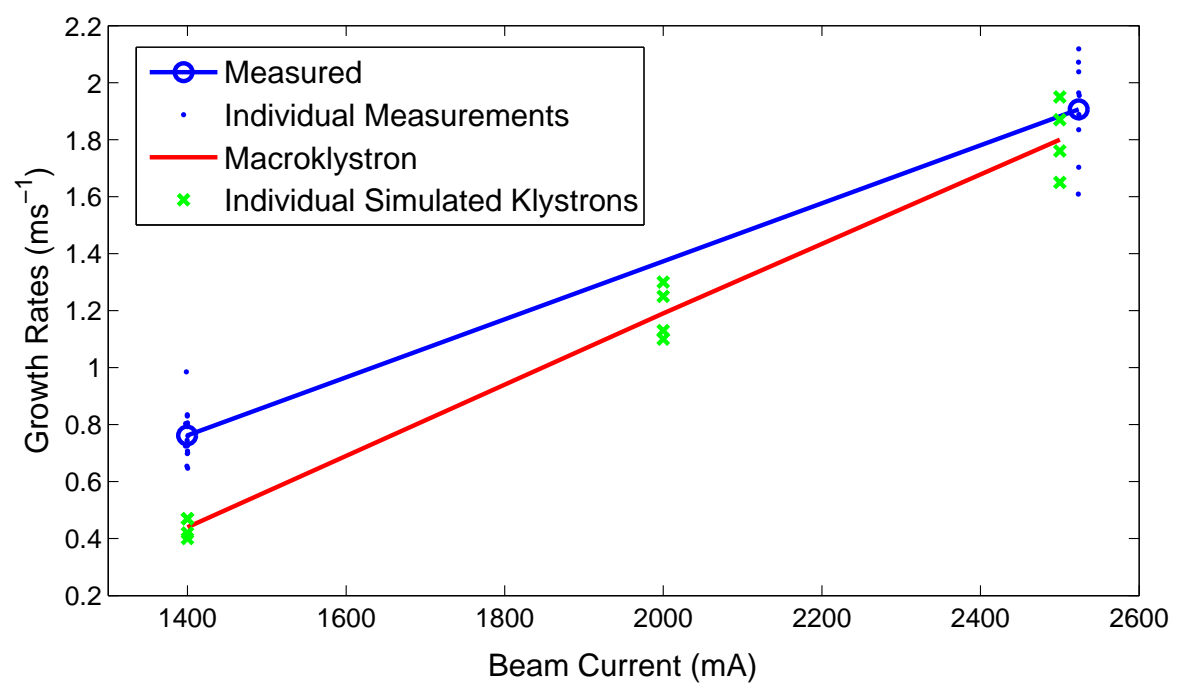

Figure 4.10: Measured and Simulated Growth Rates for the LER (Simulated for each station as well as the macroklystron). Most unstable mode is -3 .

measured from the physical system are compared with the simulation. The drifting of the growth rates in the physical system (which will be explained in Section 5.2) can be seen in this graph. The individual klystron and driver amplifier characteristics of each station are used as a model in the simulation to calculate growth rates. This process is repeated for each station, and the individual results are depicted in Figure 4.10 in green. The macroklystron is defined as the average of these results. The simulation not only reproduces the form of the most unstable growth rates for various beam currents, but it also agrees with the physical system in the number of the most unstable mode. The discrepancy at low currents (underestimation of the growth rates) motivated studies on individual system components and comparison between the simulated and measured results. These additional dedicated measurements of the physical system led to some surprising discoveries on the performance of the klystron 
pre-amplifiers, as will be shown in Section 5.5. 


\section{Chapter 5}

\section{PEP-II Longitudinal Coupled-Bunch Instabilities Studies}

Increased stored currents affect the stability of the beam and the system's robustness to perturbations. In this Chapter, we analyze the sensitivity of the coupled-bunch instabilities on LLRF parameters and estimate the operational limits due the available klystron power or the stability of the low-order mode beam dynamics driven by the cavity fundamental impedance for PEP-II. As mentioned in Chapters 3 and 4, both the RF station and the beam need to be stable for reliable machine performance. With the use of the configuration tools described in Section 4.4, the PEP-II RF station operated with sufficient gain and phase margins. At increased currents though, beam stability was an important issue for PEP-II and is the focus of this Chapter.

In Section 5.1 beam stability is defined. Based on this definition, quantitative criteria are then set in Section 5.2 to achieve beam stability. Section 5.3 presents the limits on the feasible operation points. Section 5.4 is a study of the sensitivity of beam instabilities on the various LLRF parameters and the resulting updates in configuration algorithms. Section 5.5 describes the technical imperfections of the system that were discovered with the time-domain simulation and the steps to improve them. Section 5.6 reports the current limits due to beam instabilities. Finally, Section 
5.7 presents the achieved improvement in performance due to the technical upgrades stemming from these discoveries.

\subsection{Defining System Stability}

In a dynamic system, as a particle accelerator, the operation point must be a stable equilibrium point. For a given stable operation point, there is a bounded space where the state variables of the system can be perturbed and a bounded system trajectory returns back to the original operation point. It is important to note that even for a stable operation point, the system can lose local stability if a perturbation transiently moves the operation point away from this bounded space. Therefore, knowledge of the system parameters and nonlinearities that define stability and robustness to perturbations is necessary to determine the optimal operating point.

\subsubsection{Operation Point Stability}

The interaction between the beam dynamics and the RF station impedance makes the equilibrium point unstable in absence of feedback damping at the operating currents in PEP-II. The main source of instability is the RF cavity impedance, which destabilizes beam modes from -10 to +10 . To reduce beam instability, the station impedance is minimized for the low-order beam modes using impedance control feedback in a configuration combining direct and comb filter loops. The design goal is to reduce the interaction between the beam and the fundamental cavity impedance thus minimizing the growth rates of the fastest unstable modes (usually modes -3 or -4 depending on the operation point). The gain of the feedback loops though is limited by the klystron saturation characteristics and the loop delay.

The resulting low-mode growth rates can still be positive, even after the application of the direct and comb loops. The beam can be further stabilized by the action of an additional low-mode feedback loop, the LGDW. This system has fundamental limits from the inherent group delay ${ }^{1}$, as shown in Section 4.6, and by the high

\footnotetext{
${ }^{1}$ The control filter in the LGDW is a programmable FIR filter with a $9.89 \mathrm{MHz}$ sampling rate (72
} 
frequency characteristics of the control filters.

From this description of the RF station-beam dynamics interaction, it is important to notice that the low-order beam mode stability depends on the design of the LGDW and a careful design of the feedback systems constituting the impedance control of the RF station. For an optimal configuration of these systems, the beam stability is ultimately limited by the klystron characteristics.

\subsubsection{Operation Point Robustness To Perturbations}

For the control of low-order mode beam motion the robustness of the system to perturbations is associated with the maximum transient forward power that the klystron can apply to the cavities. This maximum value depends not only on the maximum power that the klystron can dissipate but also on the saturation characteristics, as will be shown in Section 5.2.

The different power out/in characteristics in Figure 5.1 correspond to different settings of the klystron High Voltage Power Supply (HVPS). To run consistently in a relatively linear region, the input power is kept in the range between $15 \mathrm{~W}$ and $20 \mathrm{~W}$ for all PEP-II klystrons, independent of the manufacturer. This is achieved by the klystron saturation loop of Figure 4.1 which regulates the HVPS value based on the average value of the klystron input power. As greater power is demanded, the HVPS level is increased (increasing the large signal gain of the klystron), essentially moving the operation point vertically on the plot up to about $900 \mathrm{~kW}$ when the maximum HVPS voltage is reached. A further increase of output power above $900 \mathrm{~kW}$ requires increases in input power and leads into saturation, which effectively changes the amplitude and phase modulation gains differently.

Therefore, an increase in the input power for a constant forward power, leads to a lower transient maximum power and margin. The maximum klystron forward power is operationally unsustainable since the small-signal gain goes to zero. The klystron saturation limits the klystron power margin and is significant in defining the

samples/turn) and up to 32 FIR coefficients per macrobunch. The control filters employed have an effective group delay of $66 \mu \mathrm{s}$ (HER) and $108 \mu \mathrm{s}$ (LER). Filters with narrower bandwidth (greater noise rejection) could have group delays up to $141 \mu \mathrm{s}$ [30], [31]. 


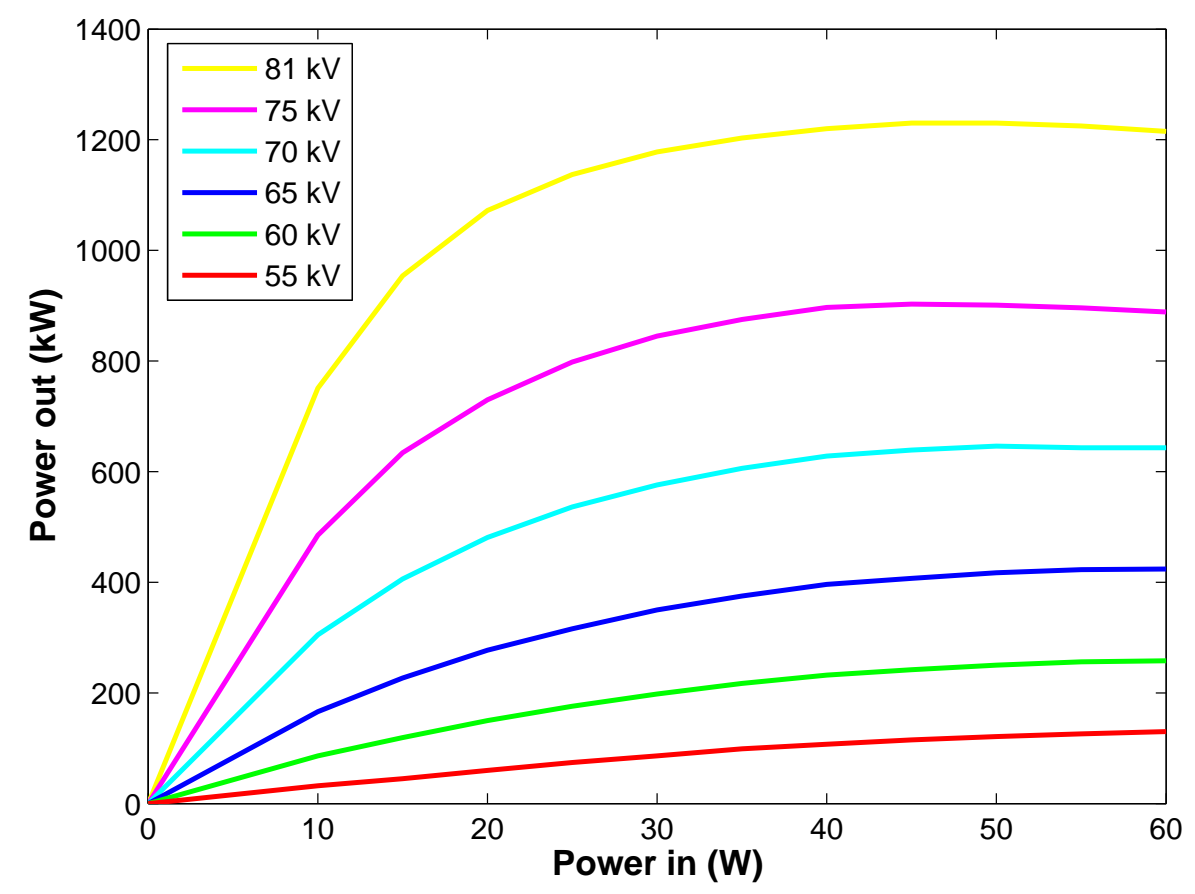

Figure 5.1: Power Curves for SLAC \#5 klystron. For a desired output power the operation point is defined by the HVPS level and the input power.

robustness to perturbations around a given operation point.

The klystron saturation also reduces the effective gain of the system, leading to reduced impedance control through the feedback loops. To achieve a compromise between the saturation effects and the available power, the maximum operable klystron forward power is decreased by $15-20 \%$ from the maximum klystron power, as described in more detail in Section 5.2. Effectively, this choice reduces the power efficiency in favor of improved impedance control and increased robustness to perturbations.

This trade-off has been studied using the simulation, but has also been demonstrated in the real machine. As an example, the peak current reached in the LER was approximately $3 \mathrm{~A}$ for $\mathrm{runs}^{2}$ 5b, 6, and 7. For the first case, a small increase of the current to approach $3 \mathrm{~A}$ led to a considerable increase in the rate of aborts. On the

\footnotetext{
${ }^{2}$ Historically, a PEP-II run corresponds to roughly a year of operation. Run 5 covers operations from May 2005 to October 2005, run 5b from November 2005 to August 2006, run 6 from January 2007 to September 2007 and run 7 from December 2007 to April 2008.
} 
other hand, at the end of run 6 and during run 7 the klystron operating point was set quite differently, so that vacuum chamber heating issues from the beam currents and bunch lengths (rather than RF margins) prevented a further increase of the current [48]. This comparison shows how careful tuning of the RF stations and optimal choice of operating point can provide much higher margins, increase the robustness to perturbations and considerably decrease the rate of beam aborts.

\subsection{Stability Criteria for PEP-II}

From Section 5.1 we can see that for system stability we need to satisfy the klystron power requirements, optimize the LLRF system, and reduce the beam growth rates below the threshold set by the maximum available damping rate from the LGDW. Quantitative limits are presented in this Section. These criteria define the growth rate and klystron power limits used in this Chapter.

\subsubsection{Klystron Power Requirements, Impact of Klystron Char- acteristics}

The RF systems must satisfy several concurrent requirements. They must provide a specific accelerating voltage, deliver the necessary RF power to compensate synchrotron radiation and high-order mode losses, regulate the RF fields by filtering out perturbations and drifts, and minimize the cavity fundamental impedance. All of these requirements are directly impacted by the base klystron power transfer characteristics as depicted in Figure 5.1 through the power curves of a 1.2 MW SLAC klystron as used in the HER [49]. It is important to notice from these curves that a specific output power requirement can be met over a range of High Voltage Power Supply (HVPS) voltages and input RF powers. The choice though of the specific klystron operation point is very critical.

In operation the klystron average power limit must be set lower than the maximum specified power to accommodate high voltage power supply ripple, line perturbations which modulate the HVPS voltage, and klystron saturation effects that limit the 
klystron gain. The collective margin amounts to a $15-20 \%$ reduction of the maximum available klystron forward power. These margins set the practical maximum steady state power limits to $\approx 1030 \mathrm{~kW}$ for SLAC klystrons and $\approx 930 \mathrm{~kW}$ for Phillips/Marconi klystrons. Furthermore, from operational and hardware considerations, the maximum accepted reflected power is set to $\approx 100 \mathrm{~kW}$. It should be noted that at this power region it is difficult to tune the stations in the simulation, for the type of klystrons installed in the LER. Since this difficulty arises for a somewhat ideal system as the simulation, we expected (and experienced) worse behavior from the physical system.

For the last PEP-II run, in the HER, station 4-6 was implemented with a Phillips manufactured klystron and all the other stations were equipped with SLAC klystrons. In the LER, there were 2 SLAC, 1 Phillips and 1 Marconi klystrons installed.

\subsubsection{Beam Current Limits due to Low-mode Instability Growth Rates}

With the simulation, we can estimate the magnitude of the eigenvalues of the most unstable low-order beam modes. The beam stability margins are then determined by comparing the simulated growth rates (real part of the eigenvalues) with the estimated maximum feedback induced damping rate $\Delta_{l}$ based on the LGDW channel configuration. Our beam stability margin criterion requires that the free growth rate $\sigma_{l}$ of the most unstable mode $l$ be lesser in magnitude than the net damping rate $d_{l}$ with feedback on $\left(\left|d_{l}\right| \geqq \sigma_{l}\right)$, as shown in Figure 3.2, rather than simply $\left|d_{l}\right| \geqq 0$. Since the net damping rate is the sum of the growth rate and the feedback induced damping,

$$
d_{l}=\sigma_{l}+\Delta_{l}
$$

the criterion is equivalent to the growth rate be lesser in magnitude than half the maximum available LGDW damping.

$$
\left|d_{l}\right|>\sigma_{l} \Rightarrow-d_{l}>\sigma_{l} \Rightarrow-\sigma_{l}-\Delta_{l}>\sigma_{l} \Rightarrow \sigma_{l}+\frac{1}{2} \Delta_{l}<0
$$


The maximum available LGDW damping rate for the HER is around $-3 \mathrm{~ms}^{-1}$ for the present configuration [30]. Therefore, for sufficient practical margins, the maximum operational growth rate is set to $1.5 \mathrm{~ms}^{-1}$. The maximum LGDW damping for the LER is $-6 \mathrm{~ms}^{-1}$ - as shown in Section 4.6 - so that the maximum operational growth rate is $3 \mathrm{~ms}^{-1}$.

The additional margin is necessary to allow for fluctuations of the growth rates or reduction of the damping rates due to drifts of parameters. The growth rate does not have an exact value, but fluctuates around an average value as various system parameters drift around a controlled value (e.g. in the physical system there is power supply ripple, other perturbations which modulate the system effectiveness). There are also variations in the woofer feedback system gain due to gap transient effects, and other system factors. Our experience operating these systems suggest that when the empirical limit is crossed there is an increasing probability of losing control of the beam. Therefore, our predictions are not for hard limits; rather they are operating points past which it is increasingly difficult to operate the stations and maintain control.

\subsection{Beam Current Limits due to the RF System}

The operation point considerations described in Section 5.2 limits the HER current to $2.3 \mathrm{~A}$ for a gap voltage of $18.5 \mathrm{MV}$ employed in the last two runs, or to $2.4 \mathrm{~A}$ for the maximum achievable gap voltage. Table 5.1 shows the operation point limits for the LER in a hypothetical case of 4 SLAC klystrons and for the actual implementation

\begin{tabular}{|c|c|c|}
\hline Gap Voltage (MV) & Run 7 Limit (mA) & 4 SLAC Klystrons Limit (mA) \\
\hline 4.05 & 3600 & 3750 \\
\hline 4.5 & 3750 & 3900 \\
\hline 5 & 3950 & $>4$ \\
\hline 5.5 & $>4$ & $>4$ \\
\hline
\end{tabular}

Table 5.1: LER operation point limits.

for the last PEP-II run (run 7). $4 \mathrm{~A}$ was the machine goal for the last run. The 
analysis that leads to these results is presented in this Section.

\subsubsection{HER}

A dominant limitation for an increase of the HER beam current is the available forward klystron power. The HER contains $3 \mathrm{RF}$ stations with 4 cavities each and 8 RF stations with 2 cavities each for a total of 28 cavities. A careful balance of power between the 2 and 4 cavity stations is necessary to maximize the beam current. The power distribution depends on the cavity voltages and loading angles. In our analysis, we assume that the cavities in all the 2 cavity stations operate at the same voltage $V_{2}$ whereas the cavities in all the 4 cavity stations operate at the voltage $V_{4}$. This uniformity is necessary to avoid arcing limits.

From operational experience we also set $V_{2}=1.1 * V_{4}$. It is then possible to determine the loading angle that maximizes the beam current for each gap voltage (the sum of the cavity voltages over all stations), based on the forward klystron power limit of $1030 \mathrm{~kW}$ per station and the reflected klystron power limit of $100 \mathrm{~kW}$ per cavity. The loading angle $\phi_{L}$ is defined as the total angle between the 2 cavity station voltage $V_{2}$ and the 4 cavity station voltage $V_{4}$. Essentially, the forward power of the 4 cavity stations is kept at the maximum and the loading angle is increased until the 2 cavity station forward or reflected power reaches its respective limit.

Figure 5.2 shows this optimization process for a gap voltage of $18.5 \mathrm{MV}$. As expected, the maximum beam current is achieved when the total forward power is at a maximum of $10.984 \mathrm{MW}$, near its limit of $11.33 \mathrm{MW}$. The limit of $11.33 \mathrm{MW}$ is achieved when all 11 stations operate at their maximum value of $1030 \mathrm{~kW}$. The maximum point defines the transition from the 4 cavity station limit to the 2 cavity station limit.

By repeating the same analysis for various gap voltages, the maximum achievable current is plotted versus the gap voltage in Figure 5.3. As expected, the maximum achievable current increases with gap voltage up to about $2.4 \mathrm{~A}$. At gap voltages above 18.5 MV though, all 11 stations have to operate very close to their maximum forward power, leading to unsustainable operation. At the gap voltage of $18.5 \mathrm{MV}$ 


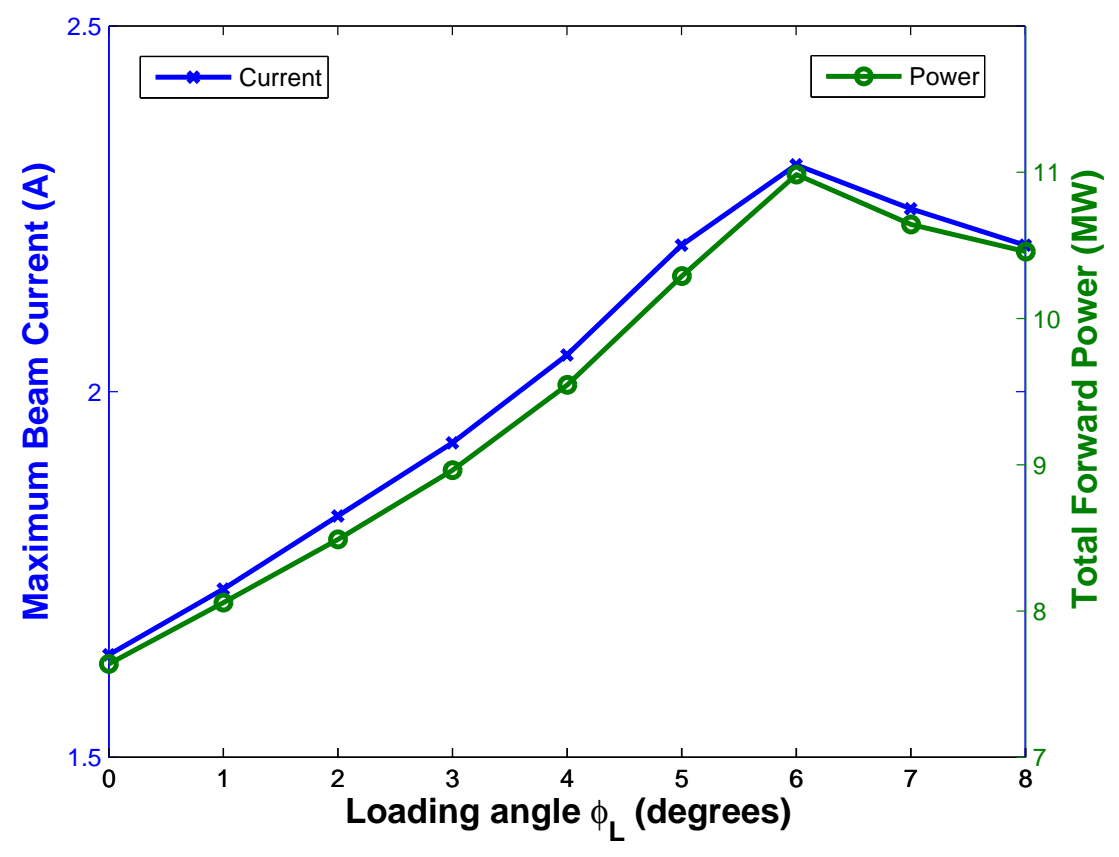

Figure 5.2: HER maximum achievable beam current and total forward power as a function of the loading angle $\phi_{L}$.

- the highest considered for run 7 -, the maximum beam current is about $2.3 \mathrm{~A}$ with $1010 \mathrm{~kW}$ klystron forward power from each of the 4 cavity stations and $985 \mathrm{~kW}$ from each of the 2 cavity stations. These limits assume that all klystrons contribute equally to the beam power. If a station must run at reduced power (due to collector cooling limits, increased HVPS ripple margin, etc.) the achievable HER current must be consequently reduced.

\subsubsection{LER}

Figure 5.4 shows the required klystron forward power per station versus current for three different gap voltages in the LER. With the existing LER power configuration, the operational total forward power as defined in Section 5.2 is $2 * 930 \mathrm{~kW}+2 *$ $1030 \mathrm{~kW}=3920 \mathrm{~kW}$ or equivalently, the average forward power per station is $980 \mathrm{~kW}$. In Figure 5.4, this limit is labeled 'Run 6 Limit', while for comparison, the 


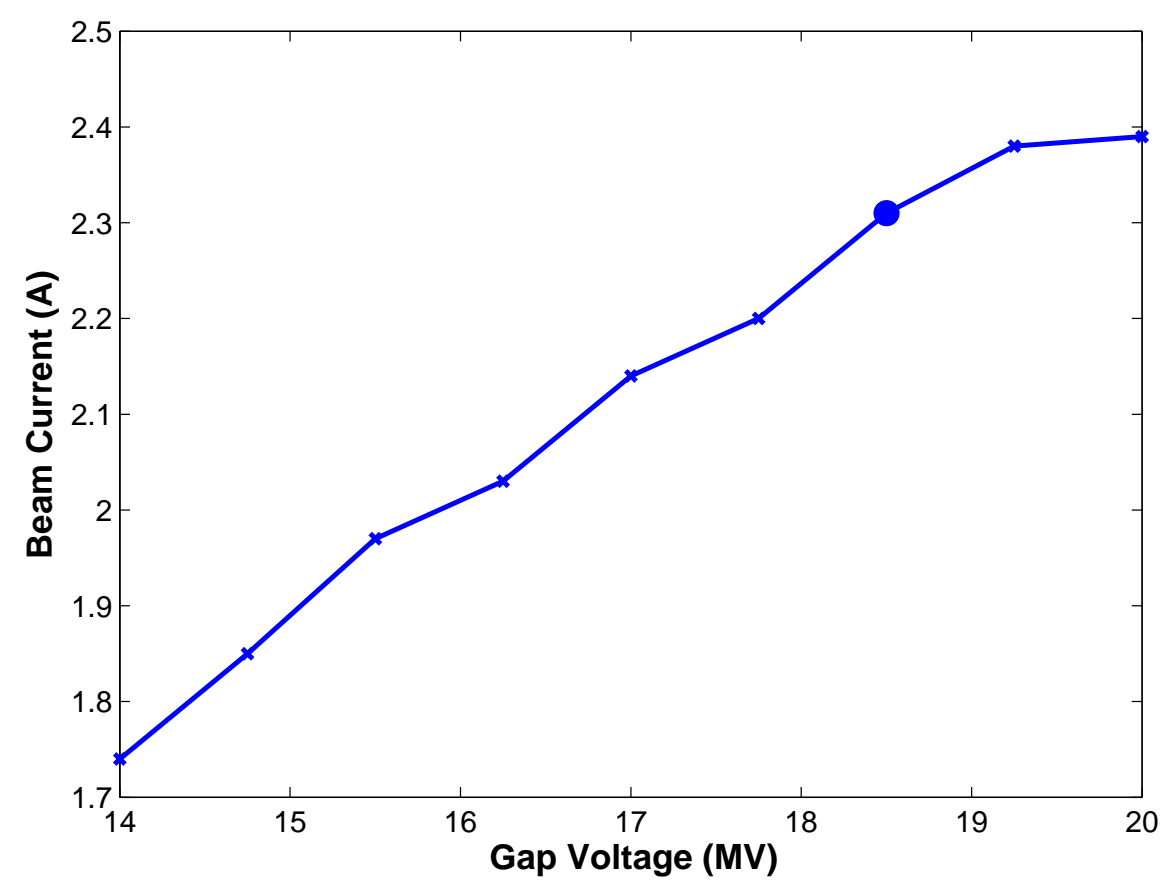

Figure 5.3: HER maximum achievable beam current versus gap voltage. The 18.5 MV operation point is marked with a circle.

line 'SLAC Limit' defines the maximum power per station if all the RF stations are equipped with SLAC klystrons. The limit is crossed at $3600 \mathrm{~mA}$ with $4.05 \mathrm{MV}$ gap voltage, at $3750 \mathrm{~mA}$ with $4.5 \mathrm{MV}$ and at $3950 \mathrm{~mA}$ with $5 \mathrm{MV}^{3}$.

During run 6 the gap voltage was set at $4.05 \mathrm{MV}$, whereas for run 7 the highest considered gap voltage was $5 \mathrm{MV}$, assuming no vacuum chamber heating problems [50], [51]. If all klystrons in the LER were to be SLAC klystrons, the limits from power considerations become $3750 \mathrm{~mA}$ and $3900 \mathrm{~mA}$ respectively for 4 and $4.5 \mathrm{MV}$. A gap voltage of at least $5 \mathrm{MV}$ would be necessary to reach $4 \mathrm{~A}$ in the LER.

In Figure 5.4 power measurements from the machine during run 6 [11] are also plotted to check the accuracy of the estimation. The theoretical estimates and the measurements have the same general form. The small difference between them could

\footnotetext{
${ }^{3}$ The necessary klystron forward power for a given current decreases with increased gap voltage due to cavity over-coupling. PEP-II cavities were designed for $750 \mathrm{kV}$ nominal voltage and are operating at $500-625 \mathrm{kV}$.
} 


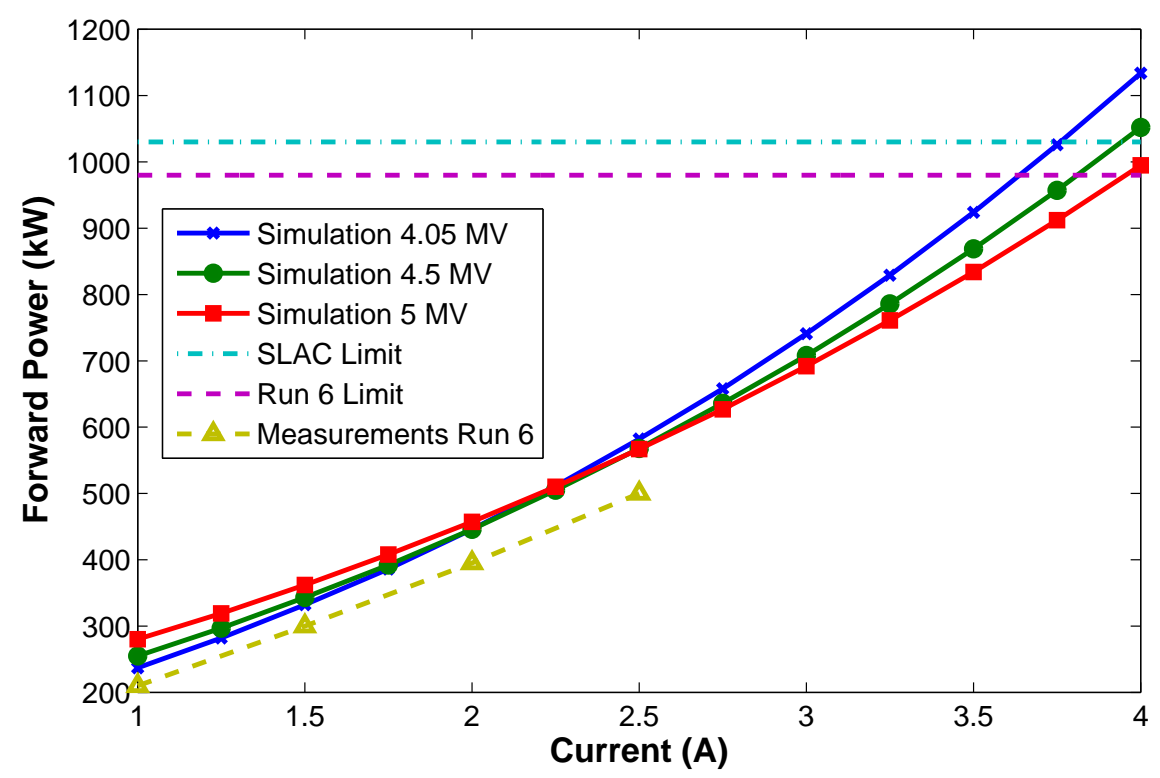

Figure 5.4: LER Klystron Forward Power versus current.

be attributed to possible calibration issues during the experiments or differences between design report values used in the simulation [52] and the actual machine parameters.

To further examine the feasibility of the 4 A LER operation and to provide insight on the gap voltage dependence and margin, the forward power is plotted versus the gap voltage in Figure 5.5. From this plot one can see that at least $4.05 \mathrm{MV}$ are needed for 3.6 A. With a gap voltage of $5 \mathrm{MV}$, operation will be marginal and unreliable at $4 \mathrm{~A}$, unless four SLAC klystrons are used in the LER (which were not considered for run 7). A gap voltage of at least 5.5 MV is required for sufficient margins at $4 \mathrm{~A}$. At the increased gap voltage of $5 \mathrm{MV}$ and higher, problems with vacuum chamber heating and high order mode structural resonance issues related to the shorter bunch length may arise [50].

\subsection{Growth Rate Sensitivity Analysis}

An important use of this simulation tool was for the study of the effect of different LLRF parameters on the beam and RF station stability and the performance of the 


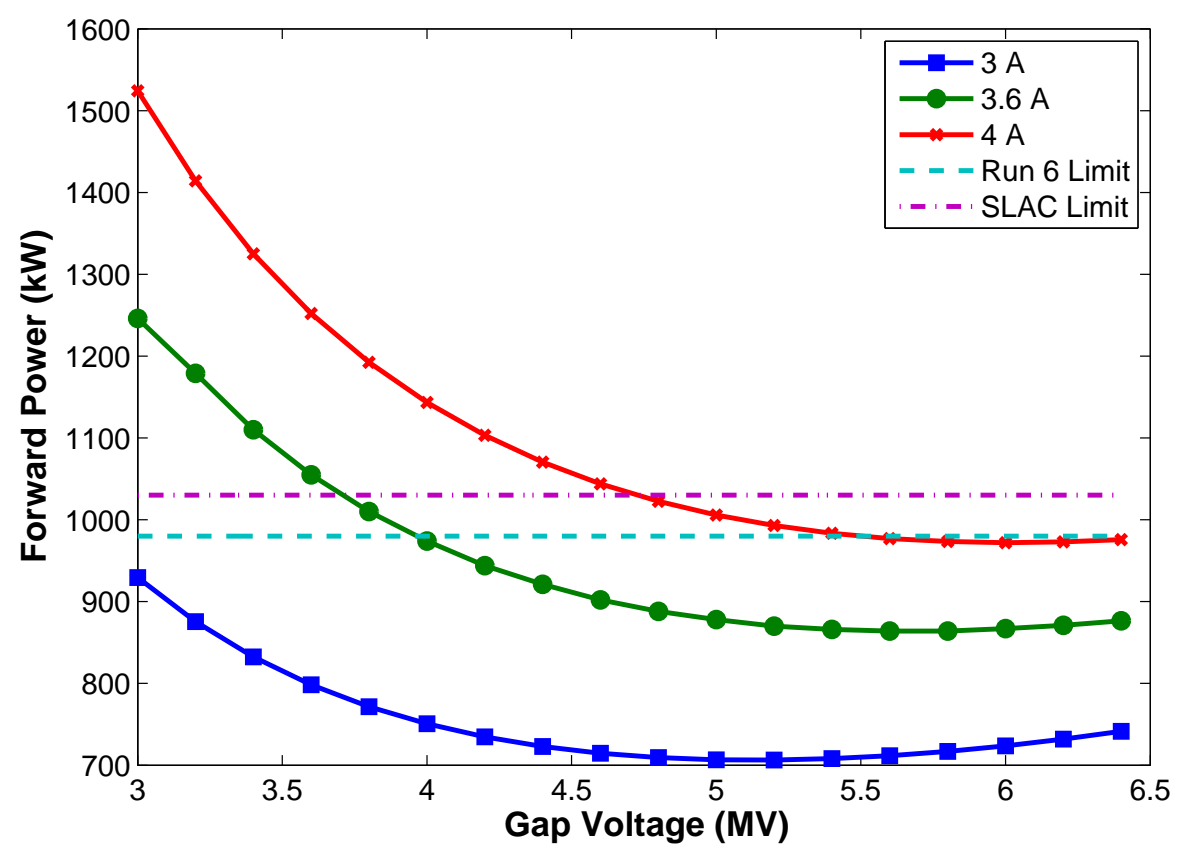

Figure 5.5: LER Klystron Forward Power versus Gap Voltage.

accelerator system, without requiring time from the operating machine. Additionally, simulations allowed analysis of different system configurations and parameter combinations that were not directly applicable to the physical machine without major system changes. These studies assisted in understanding the sensitivity of the growth rates to certain control loop parameters.

To better understand options for improved performance, the sensitivity on the adjustable parameters was considered, initially assuming an ideal linear model for the direct loop controller, driver amplifier and klystron. Based on this model, the LER operating at 4.5 MV and $1400 \mathrm{~mA}$ was simulated. The loop parameters were adjusted to satisfy the original operational criterion, that is to configure the LLRF direct and comb loops by maximizing the RF station stability margin (the direct and comb loop gain and phase margins). The growth rates and synchrotron frequency for modes -10 to 10 for the LER operating in those conditions were estimated and are depicted in Figure 5.6. The maximum growth rate, corresponding to mode -4 , is indicated by the Nominal Value in Table 5.2. By individually changing the loop 


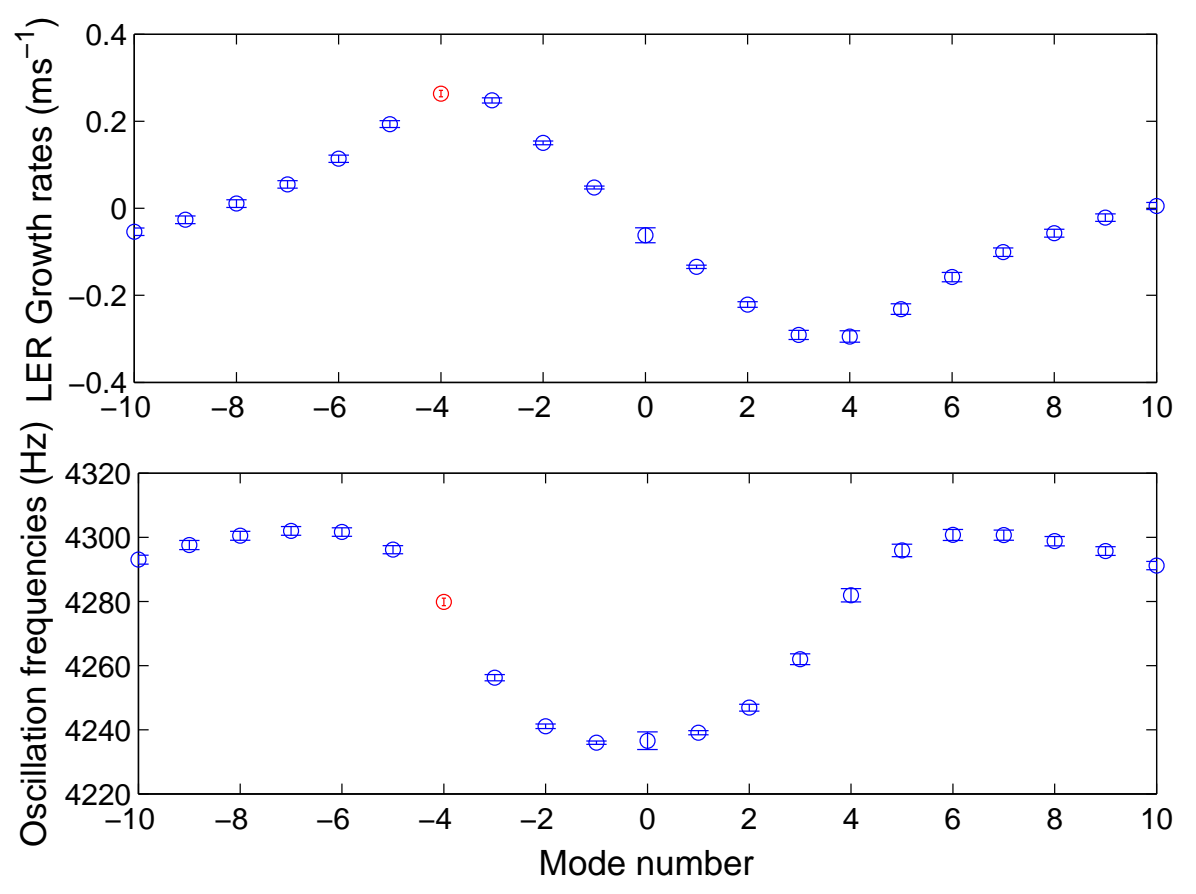

Figure 5.6: Modes -10 to 10 for nominal case.

parameters from the optimal setting, we were able to determine their impact in the interaction between the RF station and the beam dynamics. The maximum growth rates resulting from adjusting each of these parameters are shown in Table 5.2.

From these data, it is obvious that the direct and comb loop gains as well as the comb loop delay do not affect the growth rates significantly. However, the direct and comb loop phases do have a significant effect on the growth rates. This insight from the model suggests one method of influencing growth rates via adjustments of the direct and comb loop phases. The loop parameters not only influence the interaction of the beam with the RF station, but also affect the intrinsic stability of the station, as defined by $G(\omega) H(\omega)$ in Equation 4.3.

The deviation in magnitude and mode number can be seen for the direct loop phase in Figure 5.7 and for the comb loop phase in Figure 5.8 for changes of $\pm 10^{\circ}$. We can see how the rotation of the phase affects the impedance. The growth rates are reduced in the positive rotation case and increased with the negative rotation. With even larger phase rotations (not shown here) the number of the most unstable 


\begin{tabular}{|c|c|c|c|}
\hline Parameter & Adjustment & Growth Rate & Change \\
\hline Nominal Value & - & 0.263 & \\
\hline Direct Loop Gain & $+20 \%$ & 0.233 & $-11 \%$ \\
\hline Comb Loop Gain & $+20 \%$ & 0.221 & $-16 \%$ \\
\hline Comb Loop Delay & $+50 \mathrm{~ns}$ & 0.258 & $-2 \%$ \\
\hline Direct Loop Phase & $+10^{\circ}$ & 0.119 & $-55 \%$ \\
\hline Direct Loop Phase & $-10^{\circ}$ & 0.408 & $+55 \%$ \\
\hline Comb Loop Phase & $+10^{\circ}$ & 0.106 & $-60 \%$ \\
\hline Comb Loop Phase & $-10^{\circ}$ & 0.415 & $+58 \%$ \\
\hline
\end{tabular}

Table 5.2: Growth Rate Sensitivity Table.

mode changes. The margin of variation of the loop parameters to improve the beam stability is restricted by the stability margin of the closed-loop RF feedback.

These studies led to the first application of insight gained from the simulation to the physical system. The impact of the direct and comb phase rotation on the growth rates was studied in the LER. As predicted from the simulation studies, an improvement of machine growth rates is possible by adjusting the loop parameters. The simulation studies showed that the original optimal criterion to maximize the stability of the station feedback loops comes with a tradeoff to the growth rates. Figure 5.9 shows the effect on the simulated growth rates due to the direct and comb loop phase rotation. It can be seen that the optimal setting for direct and comb loop phase $\left[0^{\circ} 0^{\circ}\right]$, based on the RF station stability, does not correspond with the minimum growth rate.

We now understand that it is possible to achieve great improvement in the growth rates with a relatively small reduction of the LLRF loop stability margins. The comb loop phase rotation was studied, since it has a smaller effect on the RF station stability margins. Details of the study of the comb loop rotation are summarized in Figure 5.10, where the growth rate of the dominant unstable mode is plotted versus the comb filter phase rotation for the LER operating with $3 \mathrm{RF}$ stations at $4.5 \mathrm{MV}$ and $1400 \mathrm{~mA}$. This plot combines simulation results with the average growth rate measured from the LER operating at the same conditions. As for Figure 4.10, consistency was achieved by setting the simulation operation point so that its fit to the linear model matched 

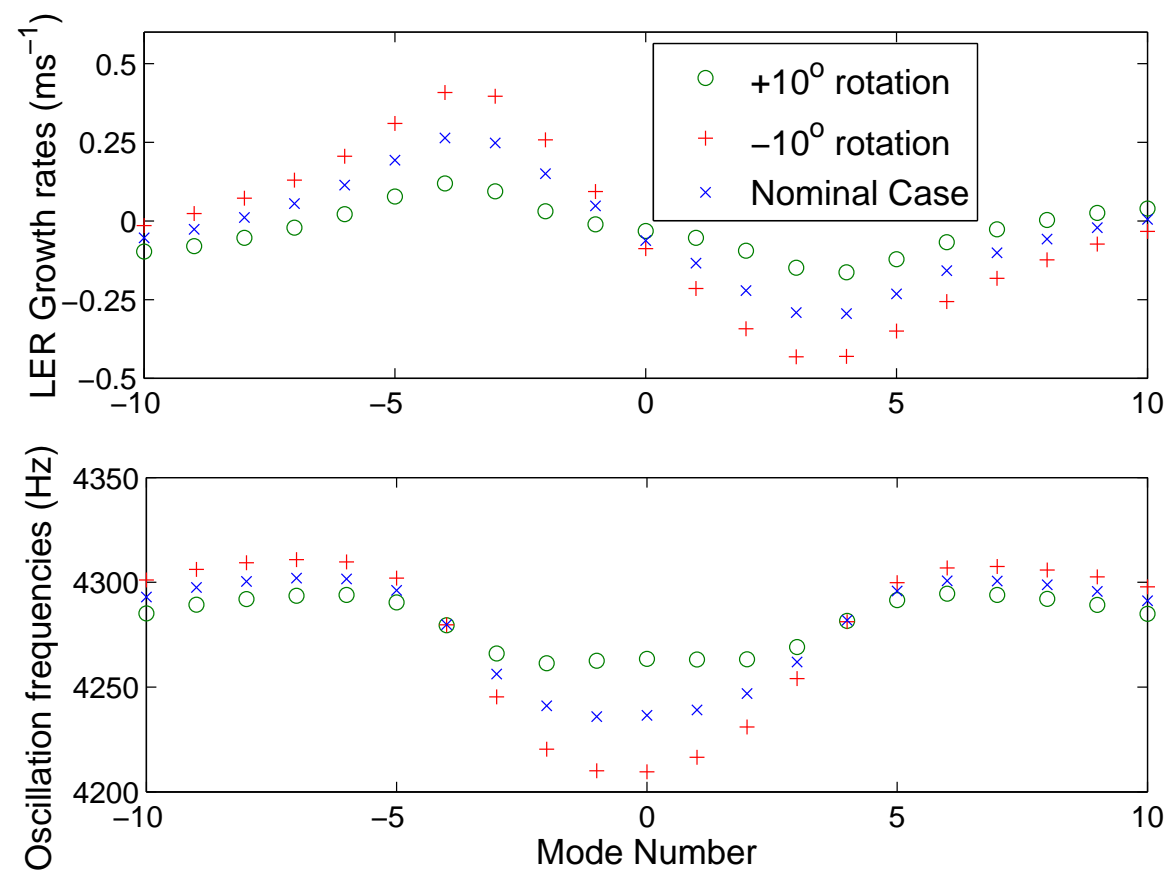

Figure 5.7: Direct Loop Phase. $+10^{\circ}$ rotation in green, $-10^{\circ}$ rotation in red, and the nominal case for reference in blue.

that of the physical system. After the $0^{\circ}$ case was checked, the comb phase was rotated by $5^{\circ}$ steps in both physical system and simulation. From the resulting figure, the optimal comb phase rotation (based on minimum growth rate) is determined to be between $15^{\circ}$ and $20^{\circ}$.

Based on these results, a comb phase rotation was applied in the LER RF systems starting on April 2006, allowing an increased beam stability margin. Only part of the optimal phase rotation as presented in Figure 5.10 was implemented, due to loss of the RF station stability margin observed in the closed loop transfer function of some stations. This effect, which did not allow implementation of the desired amount of rotation, was caused by an unexpected behavior of the driver amplifier transfer function near the carrier frequency. The source of this behavior, studied as a result of the insight from the deviations between the simulation and the physical system, is described in Section 5.5. The phase offset of about $5^{\circ}$ seen in the plot between the physical system and the simulation is possibly caused from imperfections in the 

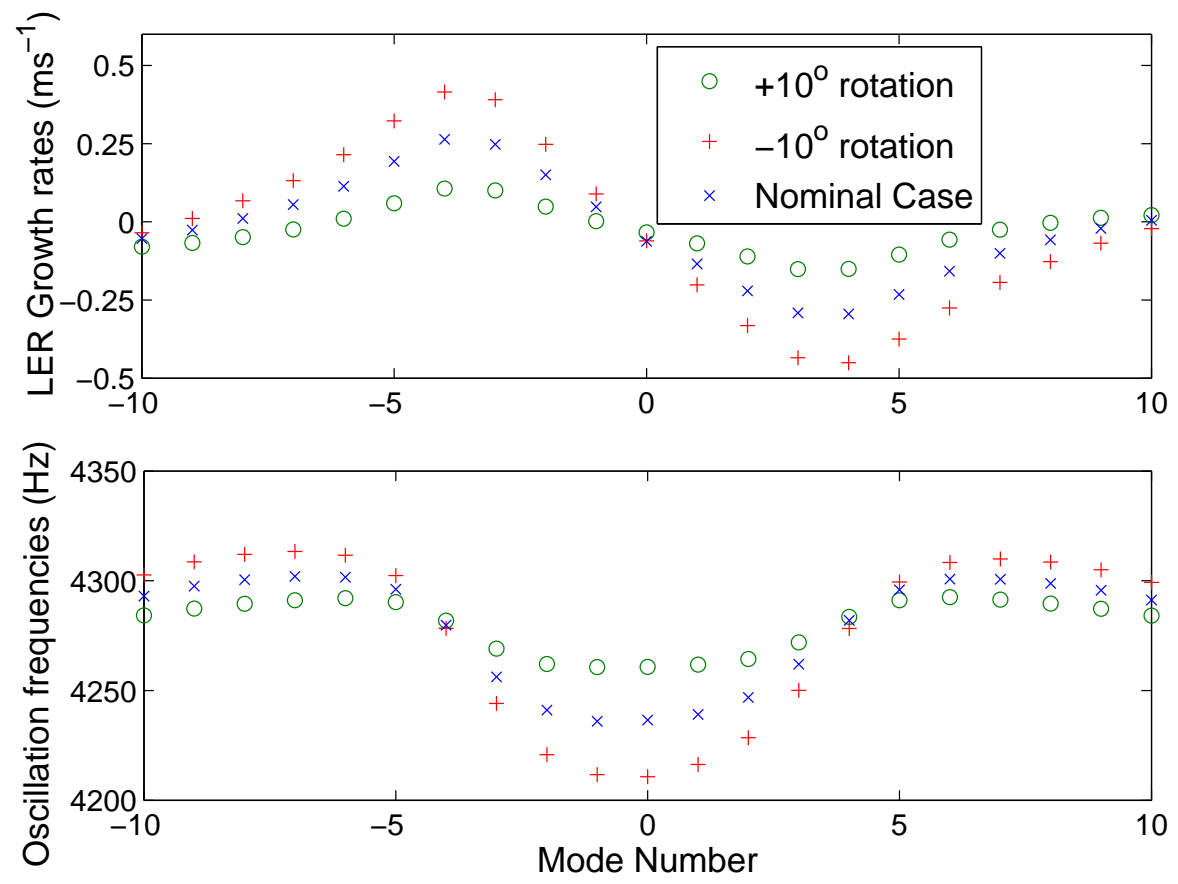

Figure 5.8: Comb Loop Phase. $+10^{\circ}$ rotation in green, $-10^{\circ}$ rotation in red, and the nominal case for reference in blue.

LLRF signal processing hardware - the radio frequency processor module (RFP).

\subsubsection{Growth Rate Dependence on Gap Voltage}

To further test the growth rate dependence on RF parameters, a study of the effect of operating with different gap voltage or number of stations at a given beam current was also studied. In this case, the station simulation model includes a non-linear klystron, an ideal driver amplifier, and the closed loop parameters are set to the optimal condition. The results are shown in Figure 5.11. As expected, we see that the growth rate drops as we increase the gap voltage per cavity. This is a result of direct reduction due to the increase in synchrotron frequency and other changes due to the operation point variations. That can happen either by increasing the total gap voltage, as seen from the first three lines, or as depicted by the first and last line by reducing the number of active stations for the same total gap voltage. 


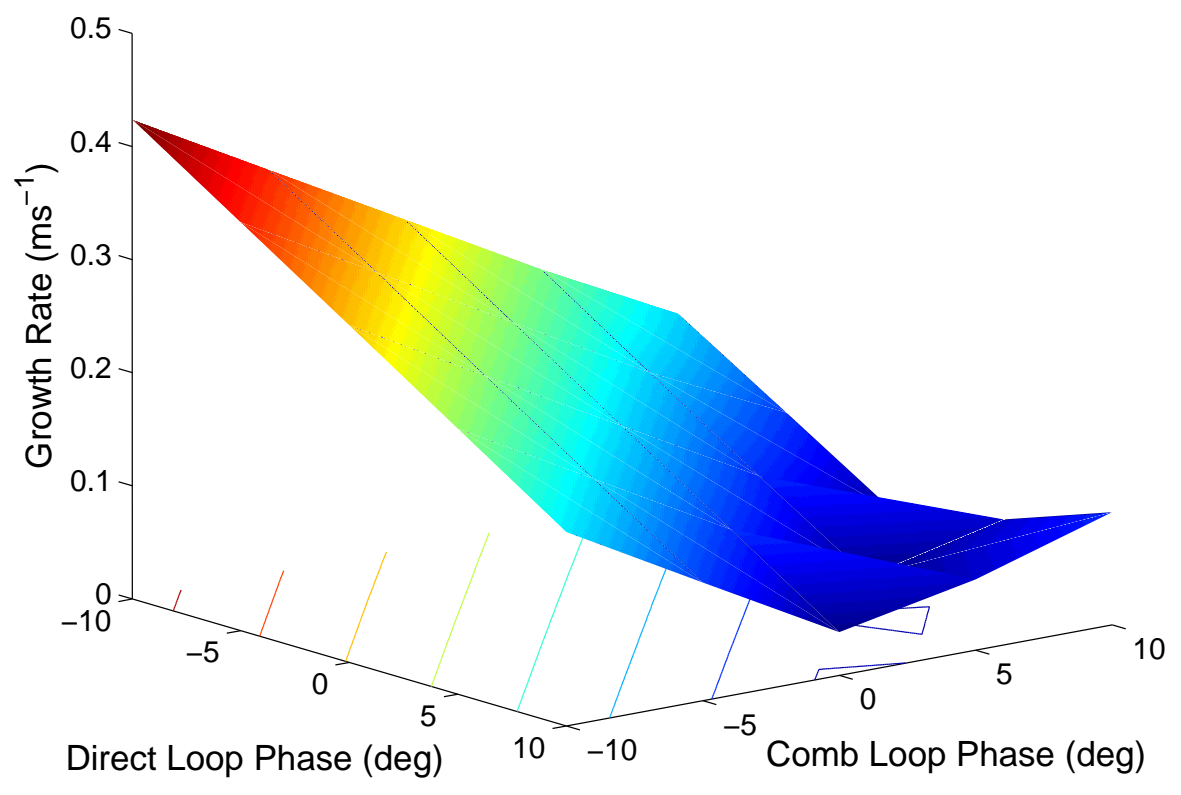

Figure 5.9: Growth Rates with Direct and Comb loop phase rotation for the LER at $1400 \mathrm{~mA}$.

\subsection{LLRF System Imperfections}

From this work, useful insight was gathered from the performance of the physical PEP-II stations and the development of the simulation macromodels. For example, it was shown that there are important variations in the frequency responses and saturation curves between stations. This is a result of the actual variations in station klystron responses, as well as variations and imperfections in the LLRF electronic systems. These variations in turn have a significant effect on the estimated growth rates.

This effect can be seen in Table 5.3 where the growth rates from LER simulated at $1400 \mathrm{~mA}$ are shown. From these data one can see the dependence of the growth rates on the klystron and driver amplifier model used. The frequency response of LER 4-2 is the furthest from desired, showing onset of RF station instability (due to its far from ideal driver amplifier as will be shown later). Consequently, it has to be operated with a lower direct loop gain $(-5 \mathrm{~dB})$ than the rest, leading to the large 


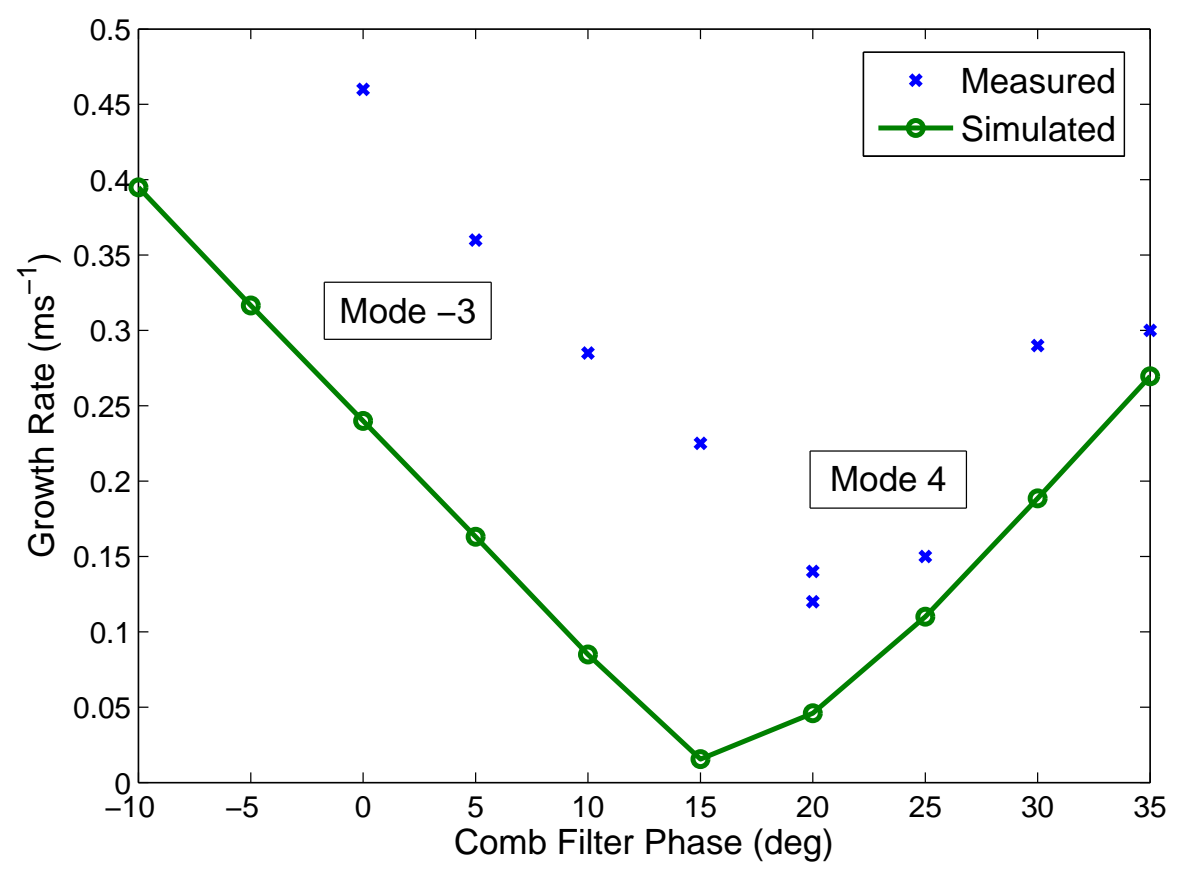

Figure 5.10: Measured and simulated growth rates vs. comb phase rotation for the LER at $1400 \mathrm{~mA}$. Agreement in both the general form and most unstable mode number.

deviation between its maximum modal growth rate and the average. The variation among stations can also be seen in Figure 4.10, for different operation conditions, where the growth rates from 4 different stations have been plotted around the value of the macroklystron. Due to these variations, a scheme had to be devised to best estimate the behavior of the physical system with multiple individual RF stations. The system's growth rates had to be computed by either averaging the growth rates from each station or through averaging the effective impedance from each station over the whole ring. This way the effective growth rate of a macroklystron that represents the whole ring is calculated.

These variations described above as well as the small discrepancies between the physical system and the simulation as presented in Section 4.7 prompted further measurements of the klystron transfer functions. In these tests a full-power klystron with low-level driver circuitry was evaluated on a test stand. The first series of tests focused 


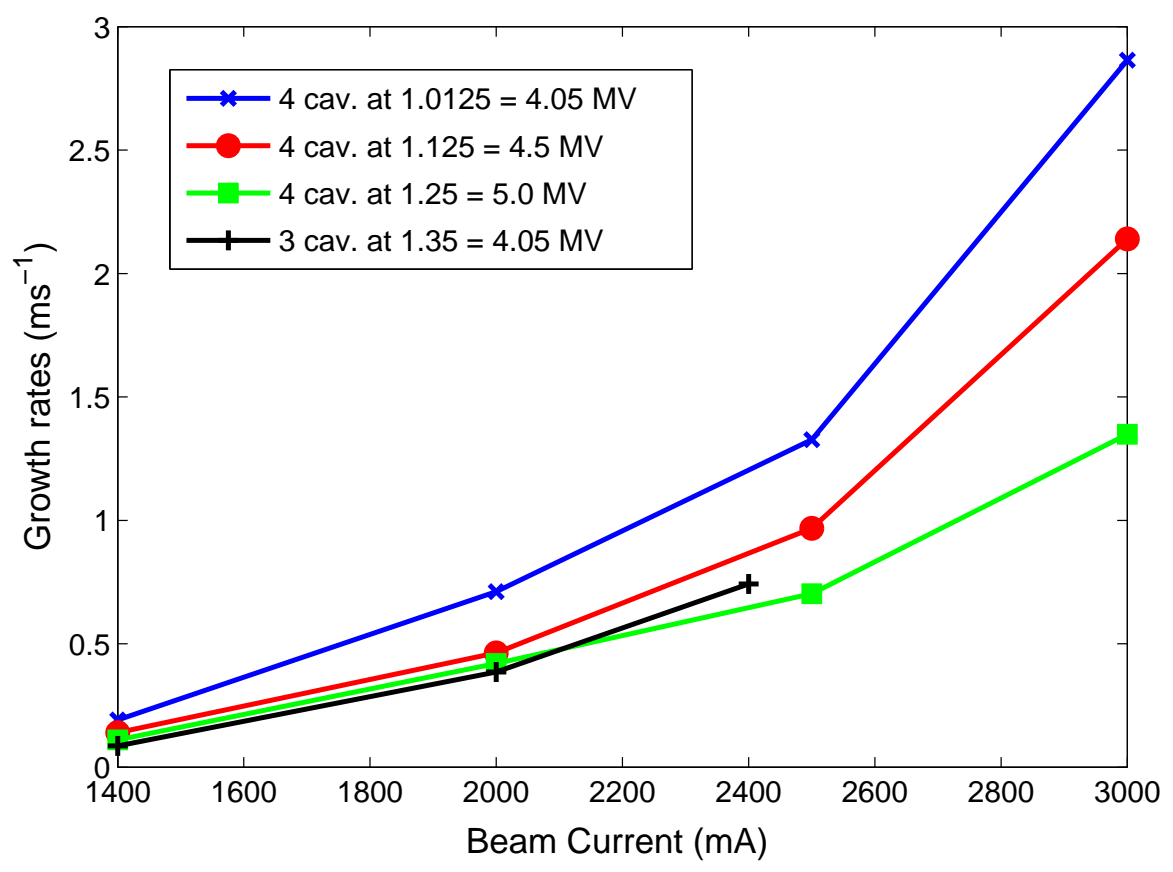

Figure 5.11: Growth Rates for different number of station and gap voltage for the LER.

on the non-linear amplitude saturation characteristic of the power klystrons. The data was used to understand the impact of this klystron non-linearity on growth rates and develop compensation techniques to correct for this effect, which resulted in the klystron linearizer [53], [54]. While there is an impact on growth rates from klystron non-linearity, it could not explain the magnitude of the deviation from expected behavior. This conclusion, together with additional measurements of the LLRF and the klystron transfer functions, led to an empirical simulation result. After a small 2-3 dB frequency response variation was added in the model klystron (in the form of a small bandpass of increased gain near the center frequency), much better agreement between the model and the physical transfer functions and growth rates was found.

A second series of full-power tests concentrated more closely on understanding the deviations, and the results showed that the apparent large deviations among klystrons were strongly related to the non-linear behavior of the LLRF system $120 \mathrm{~W}$ solid-state driver amplifier. These amplifier functions were specified and tested for 


\begin{tabular}{|c|c|}
\hline Station & Growth Rate $\left(m s^{-1}\right)$ \\
\hline LER 4-2 & 0.385 \\
\hline LER 4-3 & 0.240 \\
\hline LER 4-4 & 0.211 \\
\hline LER 4-5 & 0.260 \\
\hline Macroklystron & 0.274 \\
\hline
\end{tabular}

Table 5.3: Growth Rate vs. Station Table.

frequency response and gain uniformity in the initial development of the RF stations. However, their large-signal behavior was measured, not a small-signal measurement in the presence of a large-signal carrier. In operation, the RF station must deliver a large RF power at the carrier (ring operation frequency), but still pass faithfully the small modulation signals within the bandwidth of the direct and comb loops. It is these small modulation signals which serve to achieve the impedance control.

As can be seen from the large and small-signal transfer functions of the driver amplifier in Figures 5.12 and 5.13, when the amplifier is driven by just the RF carrier the transfer function is almost flat and the amplifier behavior unremarkable. However, for the two tone case of a large carrier combined with a small test signal (the way the amplifier is driven in normal operations) there is some unusual behavior around the carrier frequency. This small-signal transfer function distortion is very similar to the empirical result, and highlights the value of the simulation model in understanding the behavior of the physical systems.

Additional tests of several driver amplifiers installed in the LER RF stations showed that the level of the distortion varies from station to station. More significantly, LER 4-2, the station that deviated most from ideal response, is seen to have the most distorted small-signal gain characteristic. The corresponding data for all the stations are presented in Appendix C.

With the simulation model we predicted that improving these amplifier responses would lead to a direct decrease of the growth rates. We also predicted that it would allow for the full optimal comb rotation; thus further decreasing the growth rates (as presented in Section 5.4). Dedicated measurements will be shown in Section 5.7 that 

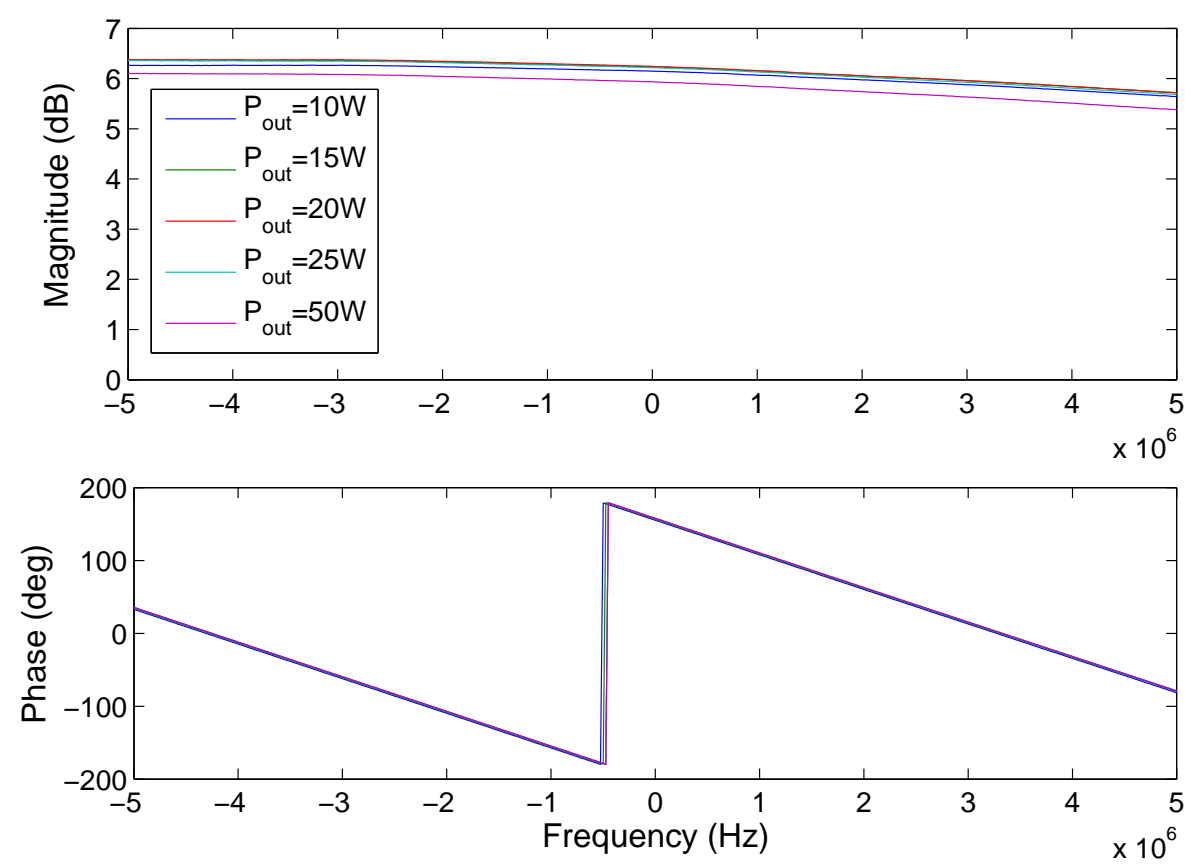

Figure 5.12: Driver Amplifier Transfer Function driven by carrier (Large Signal Response).

show good agreement with these predictions.

These results highlight that the simulation is more than a tool to imitate the physical system. It can help diagnose small imperfections and non-idealities of implementation in the LLRF systems, since it compares the physical system with the expected behavior through several physical measurements. As such, it can identify the necessity for updates or modifications to the LLRF implementation, and help evaluate possible modifications to the systems.

\subsection{Beam Current Limits due to Growth Rates}

\subsubsection{HER}

Figure 5.14 shows the growth rates for a gap voltage of $16 \mathrm{MV}$ (blue) [55]. These growth rates are estimated from the time-domain simulation data of beam motion, via 

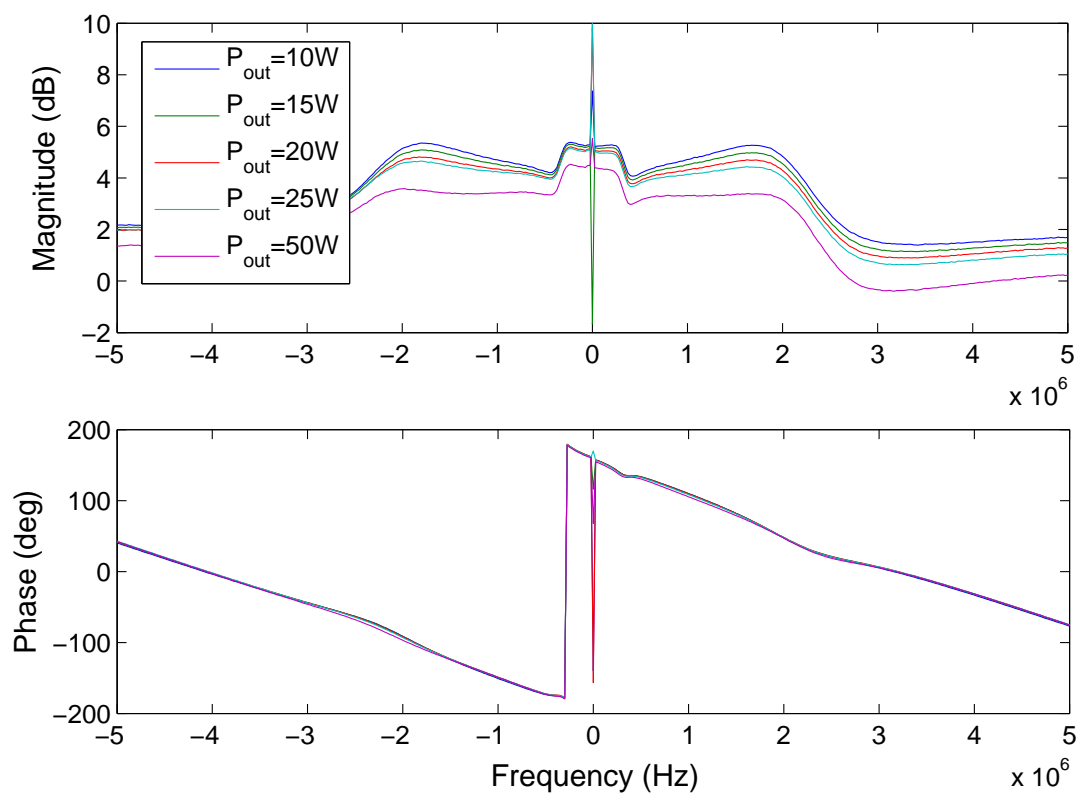

Figure 5.13: Driver Amplifier Transfer Function driven by carrier and modulation (Small-Signal Response). The modulation is swept across the band at a level $-30 \mathrm{~dB}$ below the power carrier at $476 \mathrm{MHz}$.

transformation to the modal domain and exponential fitting to the modal amplitudes versus time. These are compared and show good agreement with the measured growth rates for the same configuration in the physical machine (magenta). It is also possible to observe the variance of the measured growth rates which necessitate the margins defined above. ${ }^{4}$ In the same plot, simulated data describing the performance of the HER operating at $18.5 \mathrm{MV}$ is presented (green). This is the highest gap voltage that was considered for run 7 and shows a significant reduction of the growth rates. The 18.5 MV configuration also includes the improved klystron driver amplifiers which allowed us to achieve the desired RF station gain and phase margins. The installation of klystron driver amplifiers with a more linear response was one of the technical

\footnotetext{
${ }^{4} \mathrm{~A}$ large component of the measurement scatter reflects actual variations of the effectiveness of the impedance control techniques over time. At any given operating point, the system is continuously perturbed and the instantaneous effective impedance fluctuates due to klystron power supply ripple, line transients, beam signals, etc. all contributing to an operating point modulation. As the growth measurements are unsynchronized to these perturbations, each measurement is a snapshot of some particular operating point and resulting effective impedance.
} 


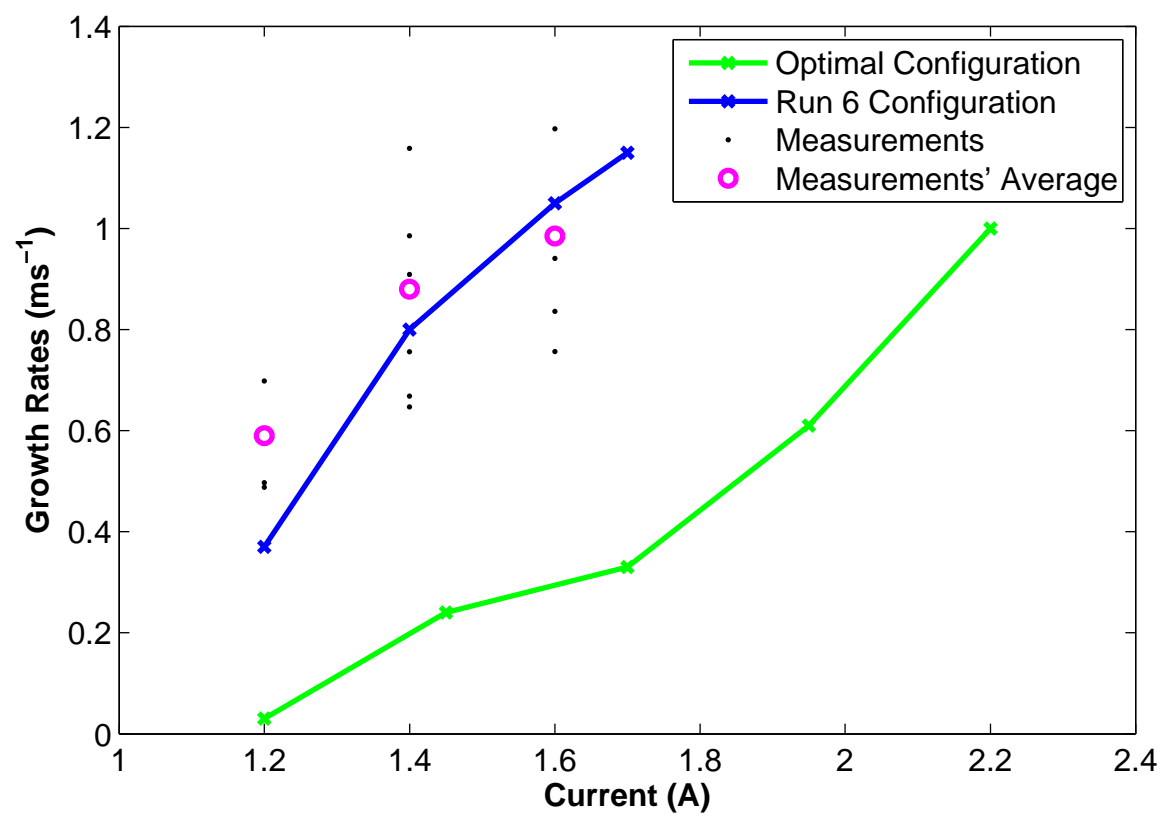

Figure 5.14: HER Growth Rates of the most unstable low-order beam mode (mode $-3)$.

upgrades implemented during run 6, as will be shown in Section 5.7.

From this curve, it is determined that the HER low-order mode growth rates do not exceed our estimated limit of $1.5 \mathrm{~ms}^{-1}$ due to LGDW damping, even for the highest planned current of $2.2 \mathrm{~A}$ with the gap voltage at $18.5 \mathrm{MV}$. As a result, the klystron forward power limit defines the maximum operational HER current to $2.3 \mathrm{~A}$ for 18.5 MV as described in Section 5.3. With the run 6 gap voltage of $16 \mathrm{MV}$ both the klystron power and the growth rate limit are crossed at about $2.1 \mathrm{~A}$.

\subsubsection{LER}

The LER coupled-bunch instabilities due to the RF cavity fundamental impedance were a significant limitation to the maximum attainable current. Therefore, possible upgrade scenarios were studied to determine what improvements would be necessary to reach the planned $4 \mathrm{~A}$ beam current for the last run 7 . The upgrades that could have significantly improved the limit included operation with four SLAC klystrons, 
the use of more linear LLRF drive amplifiers [56], and the full implementation of the comb phase rotation (a partially implemented trade off of RF station stability to growth rate improvement further discussed in Section 5.7) [7].

Figure 5.15 presents the simulated growth rates for the dominant unstable beam mode in the LER (mode -3). It depicts various LLRF configurations with LER operating at 4.05 MV gap voltage. It should be noted that as the beam current

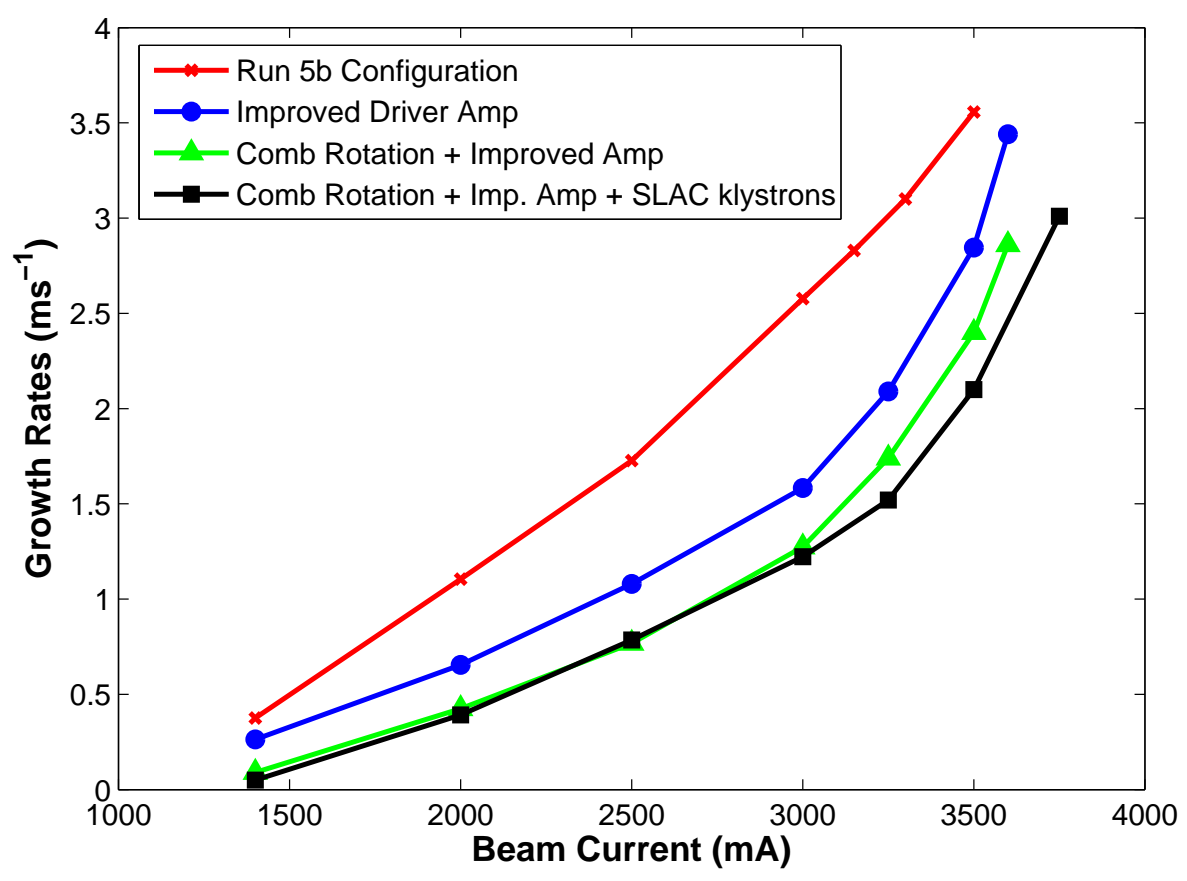

Figure 5.15: Estimated Growth Rates at 4.05 MV for the configuration during run $5 \mathrm{~b}$ and for upgraded implementations including improved klystron driver amplifiers, different comb loop phase margins, and more SLAC klystrons.

is increased in the simulation, the voltage of the high voltage power supply is also increased to match that of the physical system for similar operating points, up to the limit of $82 \mathrm{kV}$. The red curve presents the LLRF configuration used during run $5 \mathrm{~b}$, before any of the technical upgrades were implemented. The blue curve shows the reduction in the growth rates using the more linear driver amplifiers (improved driver amplifier). The green curve is a configuration with the improved amplifiers and comb rotation implemented. Finally, the black curve additionally assumes four 
SLAC klystrons in the LER.

As the current goes up, the klystron gain is reduced, especially when the HVPS has reached its maximum (highest curve in Figure 5.1). To sustain the same impedance control performance the total loop gain is kept constant by increasing the feedback gain up to its maximum value set by the RF station stability. Past this point, the effectiveness of the impedance control loop is reduced, leading to an increase in the growth rate slope, as seen after about $3000 \mathrm{~mA}$.

In Section 5.2, the beam stability criterion was defined to set the absolute value of the net damping rate higher than the growth rate so that the system is not only stable, but also immune to perturbations and drifts of parameters. Defining $m_{l}$ as the damping margin,

$$
\left|d_{l}\right|>\sigma_{l} \Rightarrow-d_{l}>\sigma_{l} \Rightarrow m_{l}=\sigma_{l}+d_{l}<0
$$

to check for the beam stability margin according to our criterion, we can plot the damping margin and look for the zero crossing. The damping margin is shown in Figure 5.16.

Figure 5.17 shows the damping margin for a gap voltage of 4.05 MV. The inflection point at $3000 \mathrm{~mA}$ in Figure 5.17 is partly due to the small increase in the slope of the growth rates, but mostly to a limitation of the LGDW channel's damping. The group delay limit of the LGDW gain sets this limit.

From Figures 5.15 and 5.17, we predicted that the limit due to growth rates for the LER operating at $4.05 \mathrm{MV}$ was at about $3150 \mathrm{~mA}$ with the run $5 \mathrm{~b}$ RF station implementation, and at $3525 \mathrm{~mA}$ with the improved amplifiers. The current limit of $3600 \mathrm{~mA}$ set by the klystron forward power, could have been reached with the combination of the improved amplifiers and comb rotation, since the growth rates still have sufficient margin at this point. The maximum achievable current with 4.05 MV is $3750 \mathrm{~mA}$ with all four SLAC klystrons and both upgrades implemented.

Figures 5.18 and 5.19 present the same configurations as Figures 5.15 and 5.17, but for the higher gap voltage of 4.5 MV. The increase of gap voltage to 4.5 MV raises the limit of the run $5 \mathrm{~b}$ configuration to $3350 \mathrm{~mA}$ and with the improved amplifiers to 


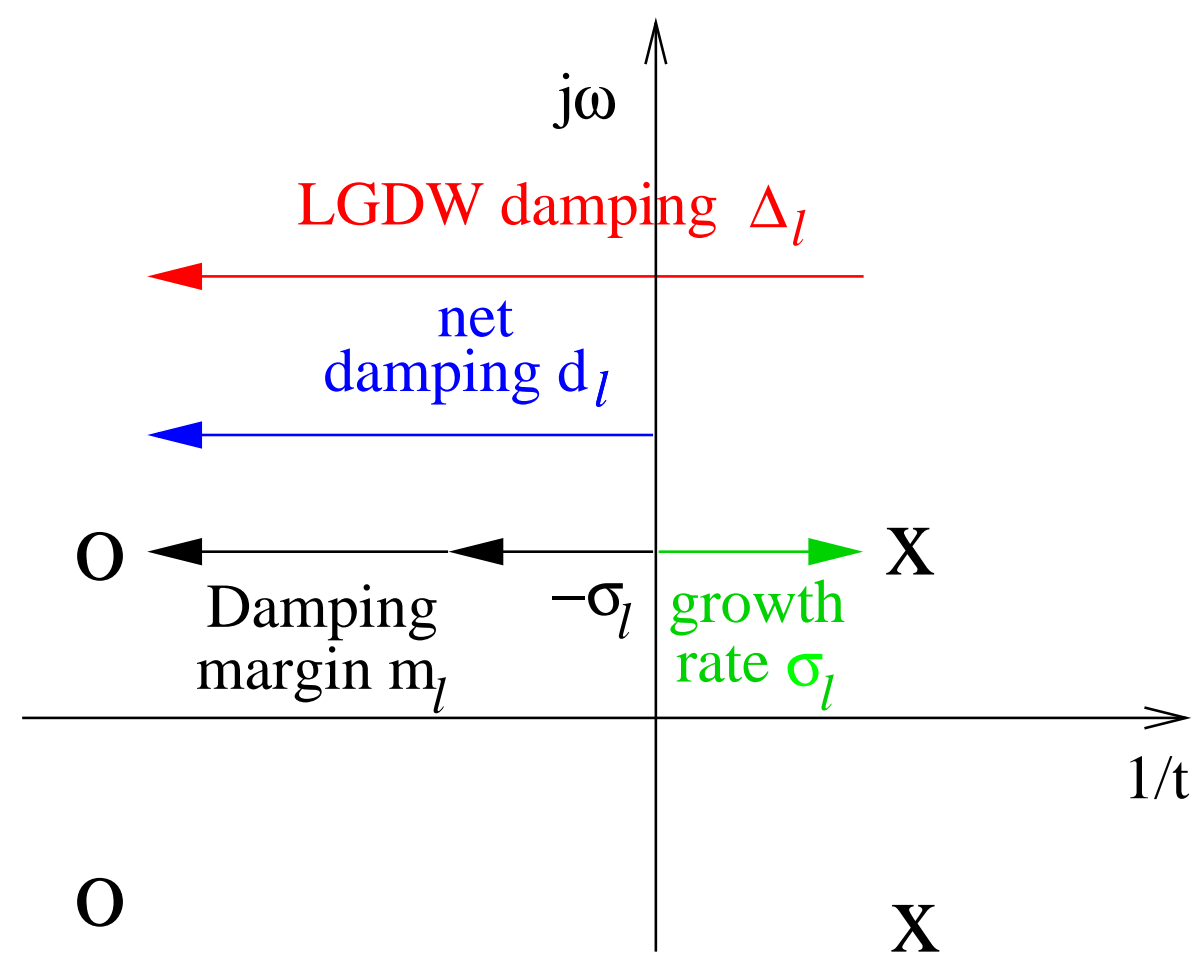

Figure 5.16: Damping margin definition.

$3725 \mathrm{~mA}$. A combination of the improvements achieves the power limit of $3750 \mathrm{~mA}$ with two SLAC klystrons or $3900 \mathrm{~mA}$ with four SLAC klystrons.

The studies above have shown that a gap voltage of at least $5 \mathrm{MV}$ would have been necessary to reach $4 \mathrm{~A}$ in the LER according to the klystron power criterion. Figures 5.20 and 5.21 show the improvement of growth rates with increasing gap voltage but also the further reduction of growth rates with the full implementation of the comb rotation. The gap voltage is set to 4.05, 4.5 and $5 \mathrm{MV}$ for the red, blue and green curves, respectively. The simulated configuration for these curves is that of the end of run 6 with the improved amplifiers and a partial comb rotation of $10^{\circ}$. The magenta curve is also at $5 \mathrm{MV}$, but with the optimal comb rotation of $20^{\circ}$ implemented, as we had planned for run 7 . The early termination of run 7 did not allow us to implement the optimal comb rotation during normal operations, but only during testing. The reduction of the growth rate with gap voltage is obvious. One can also see that the growth rates for the $5 \mathrm{MV}$ case at $4 \mathrm{~A}$ are significantly 


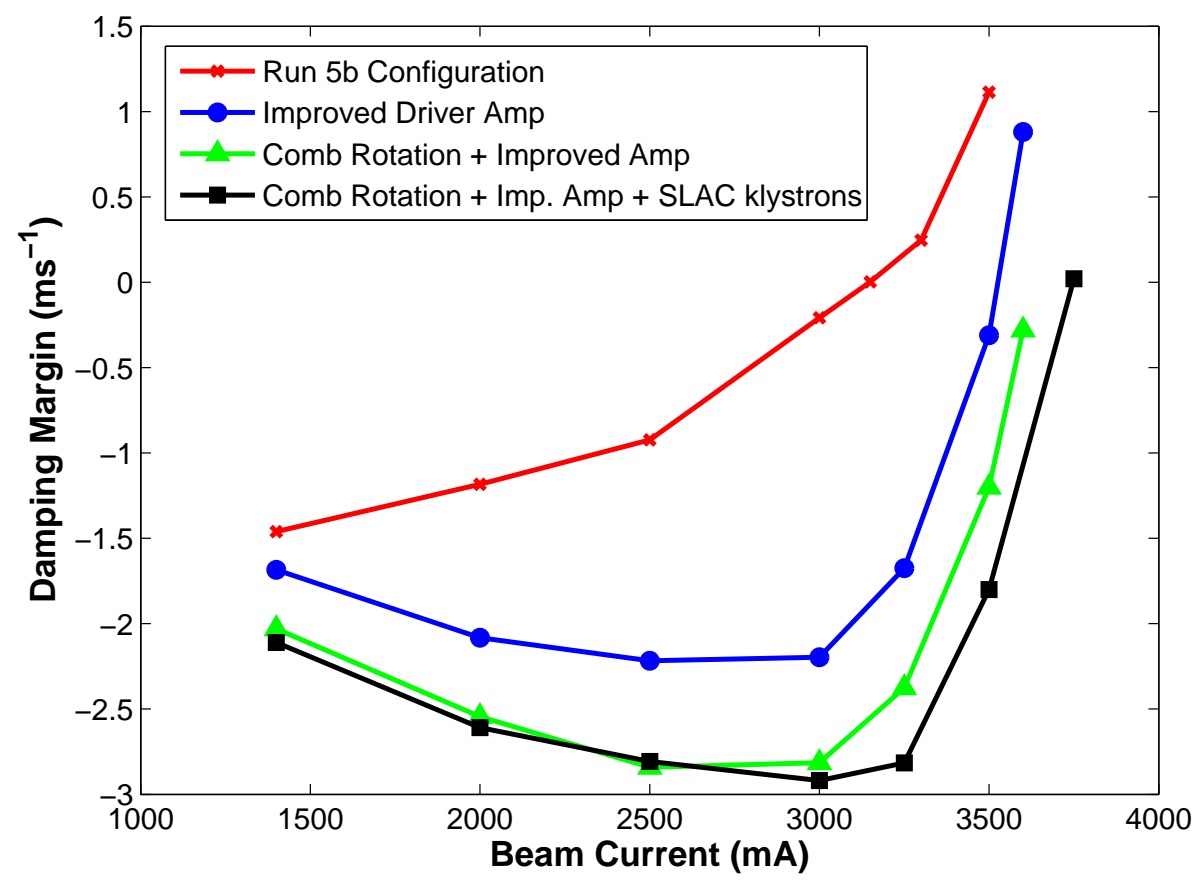

Figure 5.17: Estimated damping margins at 4.05 MV for the configuration during run $5 \mathrm{~b}$ and for upgraded implementations including improved klystron driver amplifiers, different comb loop phase margins, and more SLAC klystrons.

lower than the $3 \mathrm{~ms}^{-1}$ limit, so this current is not limited by beam stability if there is sufficient klystron power.

These results are summarized in Table 5.4, where the limiting factor (beam stability through growth rates or available klystron power) and the corresponding current is presented for each configuration. From this summary one can see that the LER situation is more difficult because both the beam and RF station limitations are reached over design currents. Higher gap voltages would have been necessary, which could have caused vacuum chamber heating and high order mode structural resonance problems [50]. Trade-offs between the limitations are necessary at high currents. 


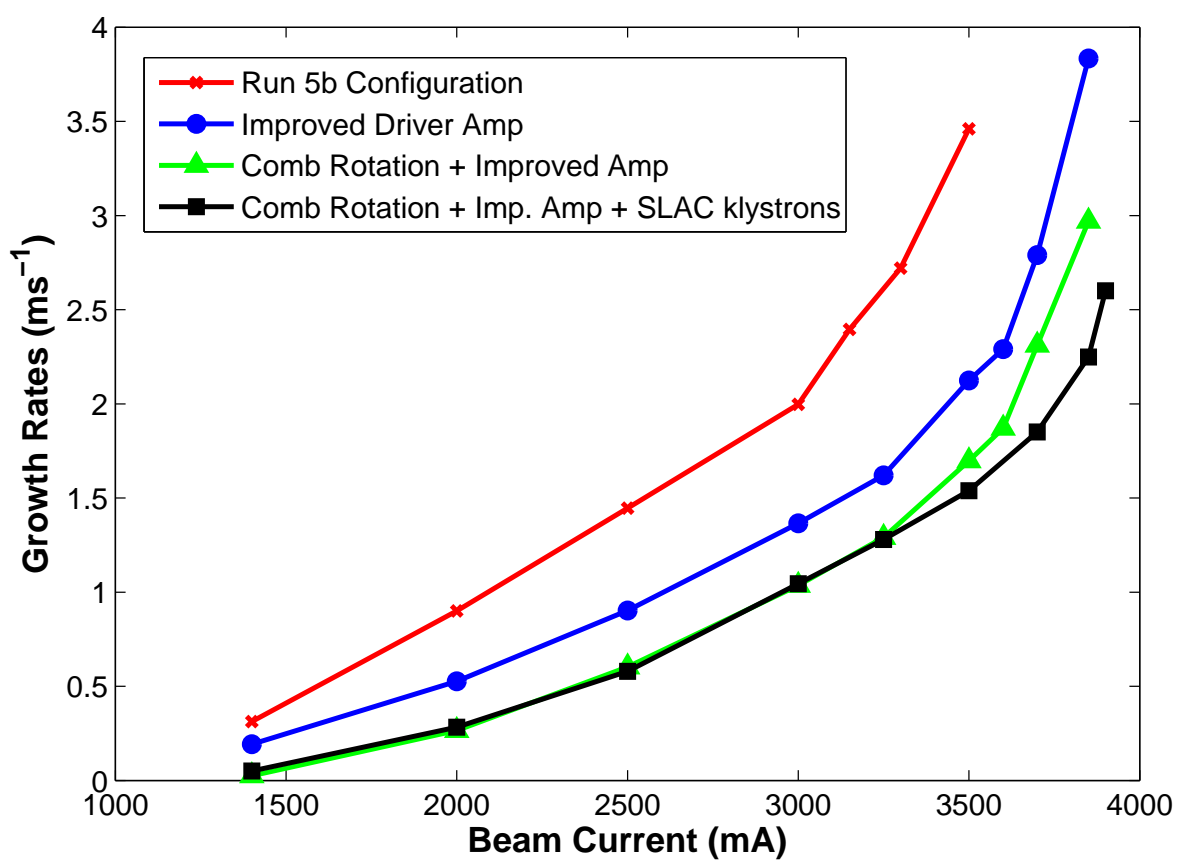

Figure 5.18: Estimated Growth Rates at 4.5 MV for the configuration during run 5b and for upgraded implementations including improved klystron driver amplifiers, different comb loop phase margins, and more SLAC klystrons.

\subsection{Technical Upgrades' Effect on Real Machine Performance}

During the development of the simulation, several technical upgrades and alternative configuration algorithms for the LLRF system were discovered and evaluated. In Section 5.4 it was shown how the RF parameter sensitivity analysis led to the implementation of the comb phase rotation. The studies on the klystron driver amplifiers presented in Section 5.5 prompted the replacement of the LLRF system $120 \mathrm{~W}$ solidstate driver amplifier. In Section 5.6, estimated growth rates from the simulation were presented to evaluate the differences among the upgrade scenarios.

After implementing these upgrades, experiments were conducted during runs 6 and 7 to prove their effectiveness and demonstrate the value of the simulation tool and its agreement with the physical system, up to the maximum current reached in the 


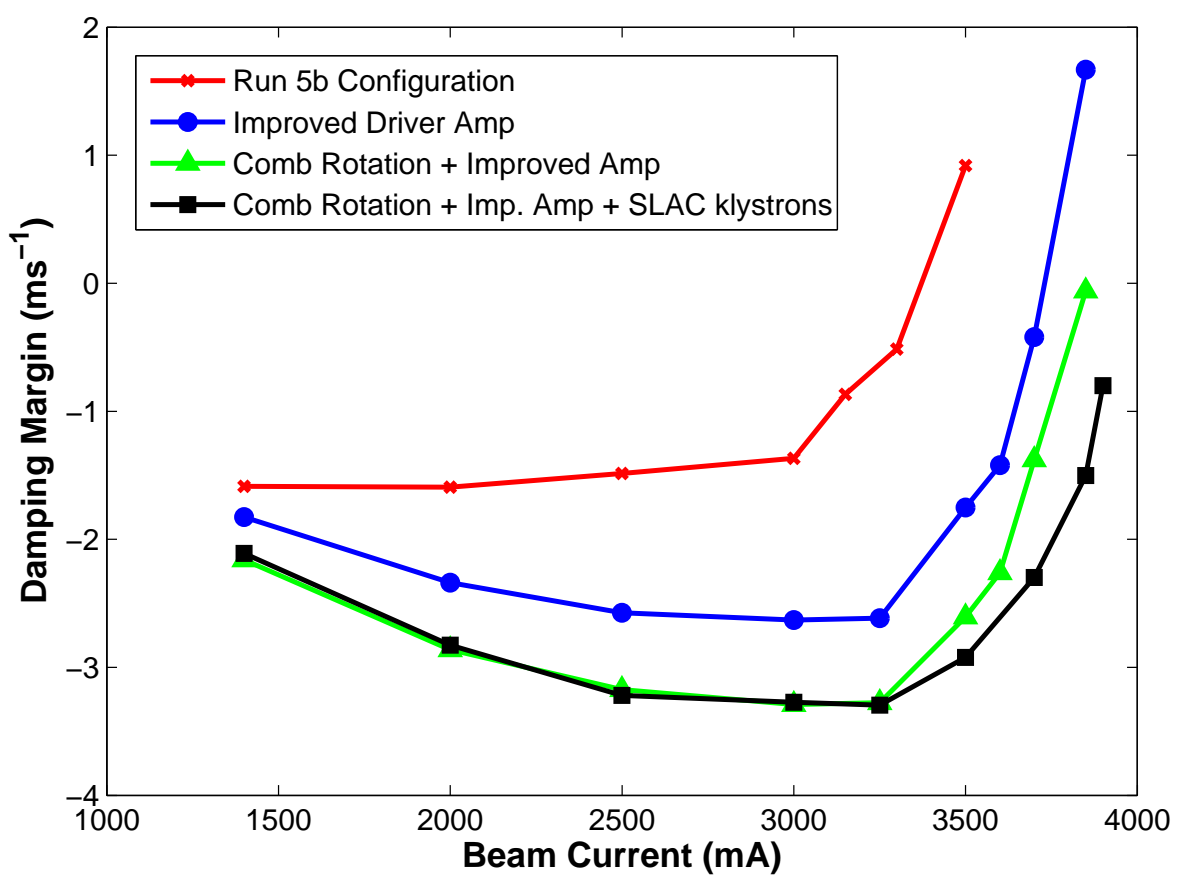

Figure 5.19: Estimated damping margins at 4.5 MV for the configuration during run 5b and for upgraded implementations including improved klystron driver amplifiers, different comb loop phase margins, and more SLAC klystrons.

LER. Figure 5.22 compares the most unstable growth rates (mode -3) from simulated data (solid lines) and measurements from the machine (dashed lines) for the LER at 4.05 MV. From the simulated data, the red curve (circle markers) corresponds to the original system configuration used during run 5b (as shown in Figure 5.15) whereas the green curve (square markers) shows a configuration similar to the end of run 6, with the improved amplifiers and the partial implementation of the comb rotation. The magenta curve (x markers) corresponds to a configuration with the improved amplifiers and the full comb rotation, that we had planned to use during run 7 . The first set of measurements in the red dashed line (circle markers) represents a configuration similar to the one used during run 5b. The blue (triangle markers) dashed curve corresponds to the run $5 \mathrm{~b}$ configuration after replacing the most distorted of the amplifiers (LER station 4-2). The black (diamond markers) dashed curve shows data taken at the end of run 6, with the improved amplifiers and partial implementation 


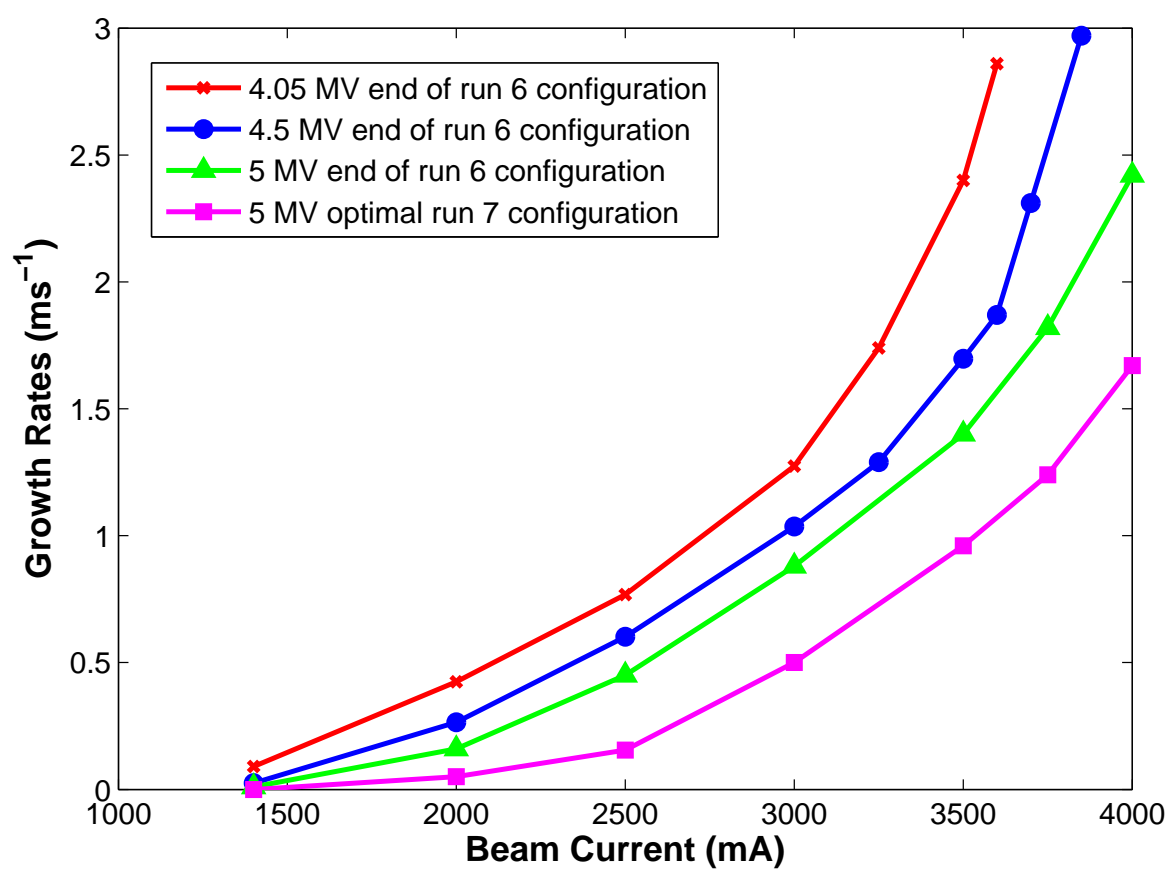

Figure 5.20: Comparison of simulation growth rates for different gap voltage settings.

of the comb rotation, but slightly reduced comb loop gain. Finally, the green dashed data curve (square markers) corresponds to measurements from the last two days of PEP-II operations, when record LER currents were achieved, with the same configuration as in the end of run 6 (improved amplifiers, partial comb rotation). These measurements show impressive agreement with the predictions for this configuration.

Comparing the red and blue dashed curves one can see the improvement by simply changing the most distorted of the amplifiers. The blue curve's growth rate measurement at $1400 \mathrm{~mA}$ is inaccurate mainly due to the short $3.5 \mathrm{~ms}$ measurement interval used, which does not allow for accurate fitting of a slowly growing exponential to the experimental data. The comparison of the green and red solid lines shows the substantial improvement expected from the implementation of the improved amplifiers and partial comb rotation by the end of run 6 . The similar comparison of the green and red dashed lines validates the simulation expectations, with the blue line providing the intermediate improvement with the replacement of the worst amplifier. The black and green dashed line share the same configuration. Their difference is 


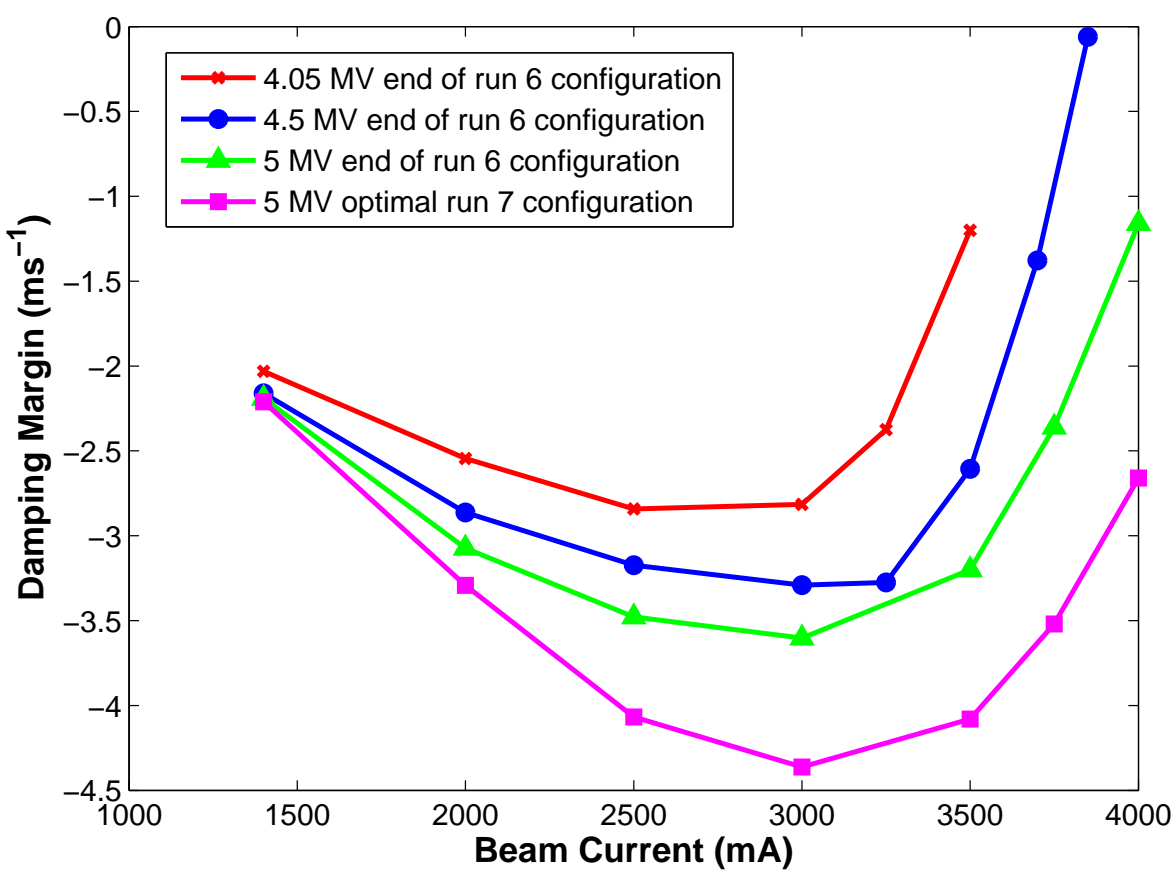

Figure 5.21: Comparison of estimated damping margins for different gap voltage settings.

partially attributed to a slightly sub-optimal setting of the comb loop gain - showing the system sensitivity to the operation point parameters -, and might be also related to the problematic operation of an RF klystron power supply at the time. One should note the large error bars of the measured growth rates - a reality of the machine that further explains the more conservative definition of limits for our predictions.

It should be noted that at the end of run 6 , with the improved amplifiers installed and the comb rotation partially implemented $\left(10^{\circ}\right.$ rather than the optimal $\left.20^{\circ}\right)$, the peak LER current achieved in the actual machine was $3000 \mathrm{~mA}$ for a short period of time. Growth rates were not measured at this current, but only up to $2900 \mathrm{~mA}$. This limit was not imposed by the RF system, but it was related to aborts triggered by the detector radiation protection, and the restricted HER beam currents that reduced the beam-beam stability effects.

Any disagreements of the estimated and measured curves can be partly attributed to the big variance of the measured data, but also to the fact that the simulation data 


\begin{tabular}{|c|c|c|c|c|c|}
\hline $\begin{array}{c}\text { Gap } \\
\text { Voltage }\end{array}$ & $\begin{array}{c}\text { SLAC } \\
\text { Klystrons }\end{array}$ & $\begin{array}{c}\text { Improved } \\
\text { Amps }\end{array}$ & $\begin{array}{c}\text { Comb } \\
\text { Rotation }\end{array}$ & Limitation & $\begin{array}{c}\text { Limit } \\
(\mathrm{mA})\end{array}$ \\
\hline $4.05 \mathrm{MV}$ & 2 & - & - & Growth Rate & 3150 \\
\hline $4.05 \mathrm{MV}$ & 2 & $\sqrt{ }$ & - & Growth Rate & 3525 \\
\hline $4.05 \mathrm{MV}$ & 2 & $\sqrt{ }$ & $\sqrt{ }$ & Power & 3600 \\
\hline $4.05 \mathrm{MV}$ & 4 & $\sqrt{ }$ & $\sqrt{ }$ & Both & 3750 \\
\hline $4.5 \mathrm{MV}$ & 2 & - & - & Growth Rate & 3350 \\
\hline $4.5 \mathrm{MV}$ & 2 & $\sqrt{ }$ & - & Growth Rate & 3725 \\
\hline $4.5 \mathrm{MV}$ & 2 & $\sqrt{ }$ & $\sqrt{ }$ & Power & 3750 \\
\hline $4.5 \mathrm{MV}$ & 4 & $\sqrt{ }$ & $\sqrt{ }$ & Power & 3900 \\
\hline $5 \mathrm{MV}$ & 2 & $\sqrt{ }$ & $\sqrt{ }$ & Power & 3950 \\
\hline $5 \mathrm{MV}$ & 4 & $\sqrt{ }$ & $\sqrt{ }$ & - & $>4 \mathrm{~A}$ \\
\hline
\end{tabular}

Table 5.4: LER limiting factors and maximum current for each configuration. The $\sqrt{ }$ mark signifies the applied upgrades in each configuration whereas the SLAC klystrons column shows how many of the four LER stations have SLAC klystrons installed (2 SLAC klystrons in the end of Run 6).

are based on an ideal driver amplifier and do not present an exact representation of the system. The improved amplifiers greatly boost the system performance, but are still not ideal. Based on initial measurements, the amplifier non-linearity, added to the distortion and non-uniformity of the Radio Frequency Processor module in the LLRF control loop (RFP) [24], could possibly impact the effectiveness of the impedance control.

The magenta curve shows the maximum possible improvement with the upgrades as we were hoping to operate during run 7 . Unfortunately, the early termination of PEP-II operations did not allow us to implement the full amount of comb rotation and measure this further improvement in the real system. The close agreement of simulated and measured data though, makes us confident that we would have achieved the additional improvement shown in Figure 5.22.

The nonlinearity of the original amplifiers did not allow us to use the optimal setting of the impedance control feedback leading to a gain reduction of the feedback loop, as described earlier in this section. In runs 1 to 6 , the direct loop gain was set $3 \mathrm{~dB}$ lower than the optimal, due to this amplifier distortion. As the improved 


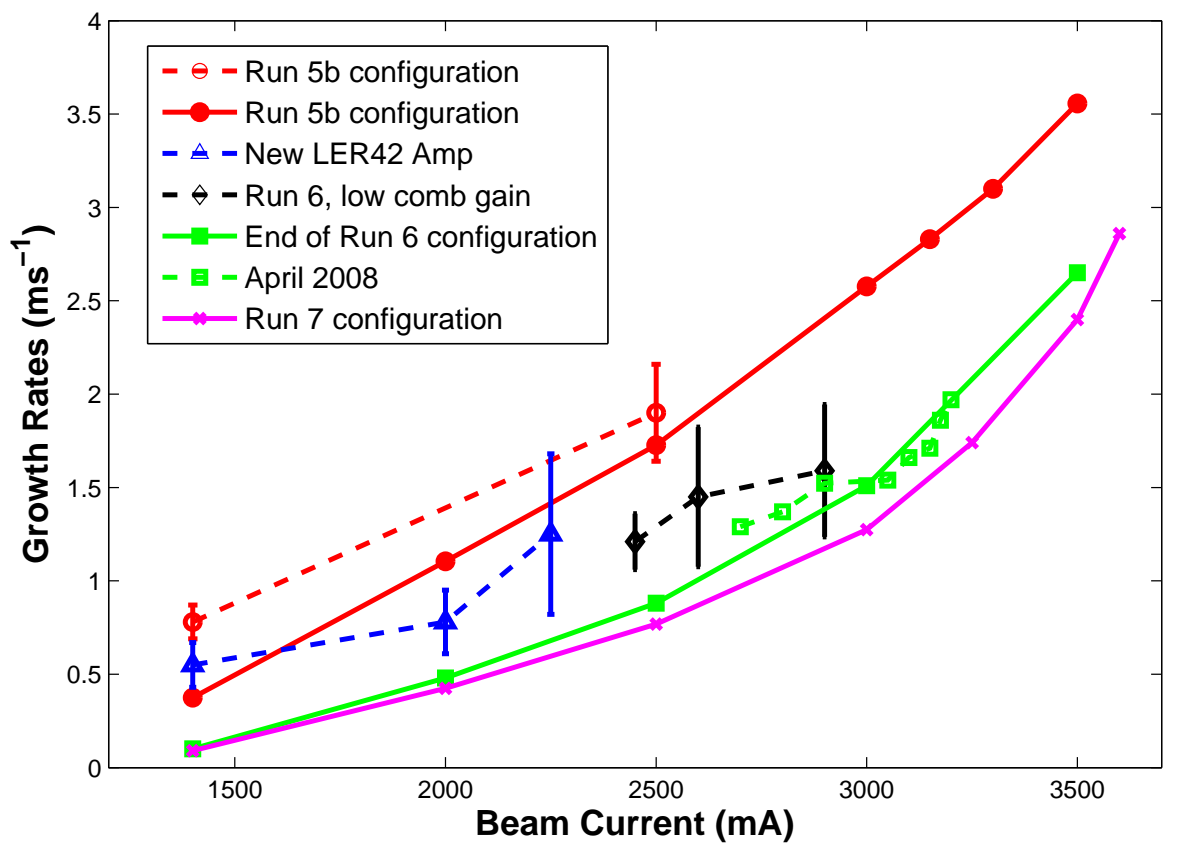

Figure 5.22: Predicted (solid lines) and measured (dashed lines) growth rates. Improvements due to the upgrades are visible. Error bars are not included for the April 2008 data because they crowd the image. They are comparable in magnitude with the error bars from the run 6 data.

amplifiers were installed the direct loop gain was increased by $1.5 \mathrm{~dB}$ to test the performance of the system. An optimal setting of the gain via a further $1.5 \mathrm{~dB}$ gain increase planned for run 7 would have further reduced the growth rates as estimated by the magenta curve.

The amplifier distortion also prevented us from fully implementing the comb rotation to the estimated optimal $20^{\circ}$, especially at higher currents. The ability to now fully apply the comb rotation provides the means to verify the simulated prediction of growth rate reduction at higher currents (low current verification in [7]). Figure 5.23 shows the measured growth rate as a function of comb rotation for the LER at $2450 \mathrm{~mA}$ and compares it with the simulation. Both the measured and the simulated growth rate decrease with comb phase rotation, as predicted. The simulated and measured data have the same functional form and slope proving the value of the tool. The simulation is an idealized system and underestimates the growth 


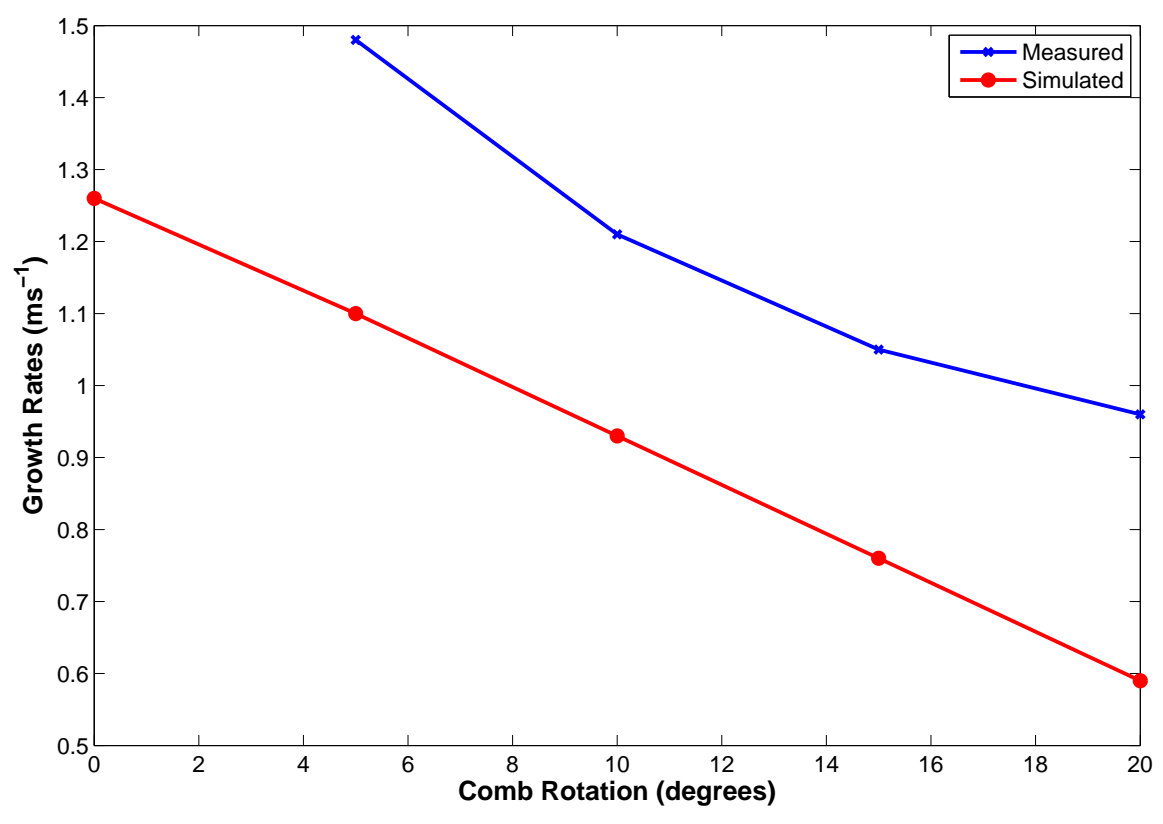

Figure 5.23: Measured improvement in growth rates versus comb rotation for LER at $2450 \mathrm{~mA}$.

rates due to residual imperfections of the LLRF from the driver amplifiers and the RFP module as described above.

Additional evidence of the distortion reduction with the improved amplifiers is shown in Figure 5.24, where we compare the LER 4-5 closed loop transfer functions (measured as shown in [7], [47]) for $0^{\circ}$ and $25^{\circ}$ comb rotation at $1200 \mathrm{~mA}$. Due to the significant distortion of the original amplifiers, the stability margin of the RF station feedback loops was greatly reduced. This effect can be seen by the peaks in the closed loop transfer function near the carrier frequency, as depicted in Figure 5.25 for $20^{\circ}$ comb rotation at $1500 \mathrm{~mA}$. The distortion resulted in reduced gain and phase margins and station instability. Consequently, the direct loop gain was reduced to satisfy the gain margin requirements. As a result, the fundamental impedance is not flattened as effectively in Figure 5.25 with the old amplifiers as in Figure 5.24 with the upgraded system. The improved amplifiers not only improve station stability but also allow us to implement the optimal value of the comb rotation for maximum growth rate reduction. It should be noted that for the initial implementation at the beginning of 


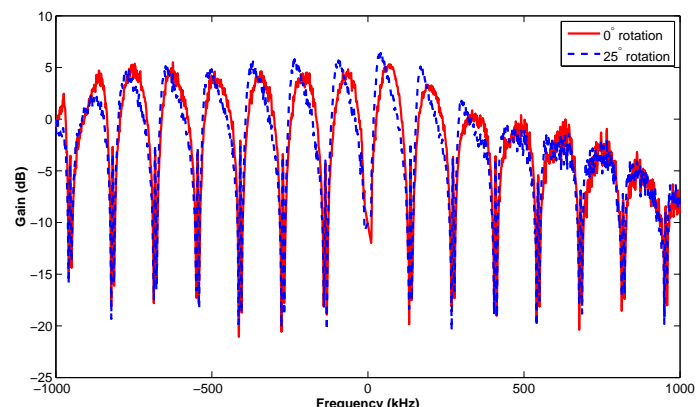

Figure 5.24: Measured Transfer function magnitude from LER 4-5 at $1200 \mathrm{~mA}$ showing minimal comb rotation induced distortion with the improved amplifiers.

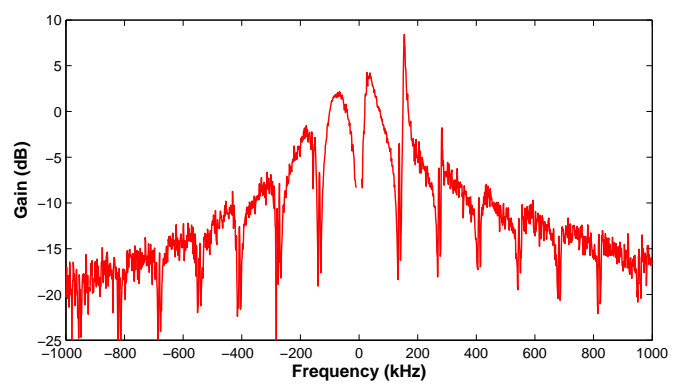

Figure 5.25: Measured Transfer function magnitude from LER 4-2 at $1500 \mathrm{~mA}$ during run 5b, showing comb rotation induced distortion with the original amplifiers.

run 6 , the comb rotation was limited to $10^{\circ}$ or even less for the PEP-II RF stations with the driver amplifiers presenting the highest distortion (for example LER station $4-2)$.

\subsection{Conclusions on PEP-II Simulation Studies}

The simulation of the PEP-II rings described in this Chapter is a close representation of the actual system. It was used to study the existing system and planned upgrades. It was also used to study and develop new operating configurations at multiples of the original design currents, as a means of better understanding operational strategies and ultimate limits of the systems. The insight gained from these studies was very helpful in the effort to optimally use the resources and available hardware to achieve the highest possible currents.

Through these studies, limiting factors and acceptable margins for high beam current operations were specified. The importance of the RF feedback loop configurations, the LGDW design, the klystron characteristics, and the dynamic stability margin has been better illustrated. In particular, it was shown that the klystron saturation not only limits the klystron power margin, but also is essential in defining the robustness to perturbations around a given operation point. 
These studies suggest that the HER could have achieved 2.2 A with a gap voltage around 18.5 MV, but even slightly higher currents would have been very difficult to reach due to klystron power limitations. For the LER with the LLRF station implementation from the beginning of run 6 and an increased gap voltage of $4.5 \mathrm{MV}$ the limit was estimated at $3350 \mathrm{~mA}$. The improved amplifiers and the comb rotation as implemented by the end of run 6 raise this limit to $3750 \mathrm{~mA}$. Finally, SLAC klystrons for all LER stations and a gap voltage higher than 5 MV would have been necessary for $4 \mathrm{~A}$, with possible problems with vacuum chamber heating and issues related to higher order modes.

With these studies, the new driver amplifiers, and the comb rotation, a world record current of $3213 \mathrm{~mA}$ was achieved at the LER. Measurements were conducted to quantify the growth rate reduction and the agreement with the predictions. With the implemented upgrades of the new driver amplifiers and the comb rotation, a $30 \%$ current increase for constant growth rates was reached. 


\section{Chapter 6}

\section{RF System Models for the LHC with Application to Longitudinal Dynamics}

In PEP-II, our models and simulation helped us push the current to higher levels, discover imperfections in RF and LLRF components, and better understand/utilize the trade-off between RF station and beam stability, as presented in Chapters 4 and 5 . The similarities between the LHC and PEP-II LLRF systems motivated the adaptation of the existing time-domain models and simulations to the LHC implementation to study both single-bunch and multi-bunch beam dynamics, as well as the dynamics of the station. The goal was to verify high-current and upgraded operating conditions of LHC, study optimal configuration techniques, estimate limits of the system, and the possible impacts of technical aspects of the implementation.

The initial motivation for the LHC time-domain simulations and models is presented in Section 6.1, Section 6.2 describes the similarities between the two systems, and provides a short summary of the LHC implementation. The modeled components are introduced in Section 6.3. The initial validation results of the LHC simulations are shown in Section 6.4. 


\subsection{LHC Simulation Motivation}

Section 4.4 presented the importance of a method to characterize and configure the LLRF system to minimize the effective impedance experienced by the beam, but also to satisfy stability requirements for the RF feedback loop. Similar tools were necessary for the commissioning and operational control of the LHC RF system. After discussion with the CERN BE-RF group, development of these tools started in late 2007 based on the PEP-II experience. The configuration tools became a necessity after the LHC incident during the initial commissioning of the machine in September of 2008 and the subsequent stricter CERN policies which prevent tunnel access when the magnets are energized. The time-domain simulation was used in the development of the LHC identification and configuration tools, and the related models and measuring techniques were part of the software suite delivered to CERN. Section 6.1.1 provides a summary of the identification and configuration tools. More detailed analysis has been presented in [57] and [58].

With the time-domain simulation and related models modified for the LHC implementation, it was now possible to evaluate the regulation of the cavity signals, study the necessary technical specifications of the various sub blocks, understand how the technical implementation impacts the system performance, predict limits of accelerator performance, and consider the effect of possible modifications and upgrades. More importantly, it was possible to study the LHC longitudinal beam dynamics, both single and multi bunch effects.

\subsubsection{Identification and Configuration Tools}

The LHC identification and configuration tools allow to optimally configure the RF stations and remotely access the RF system for tuning and measurements. During operations the tools help compare the machine's performance with the one predicted by the simulation. The fundamental techniques employed are common to the PEP-II configuration tools presented in Section 4.4. The differences in the two implementations are related to the LHC klystron characteristics, the much higher quality factor for the superconducting cavities, and design variations in the LLRF system. 
The LHC RF station configuration and measurement of variables can only be conducted remotely during operations, due to the location of the stations. The LLRF controller's parameters for each station are calculated based on an analytic model of the system. This model is dependent of the operating point of the RF station and is measured and calculated through an identification process. The identification tool measures the transfer function of the RF station in open loop during commissioning and in closed-loop when the system is operating with beam in the machine.

The identification tool operates by injecting a band-limited low-level noise signal into the system. The time-domain input signal and the response of the RF station to that excitation are measured simultaneously. The transfer function at the specific operation point is estimated using a correlation algorithm between the input/output time-domain signals. To get an analytical representation of the estimated transfer function, a linear reduced model of the RF station is fitted to the estimated data. This model is the base for the design of the LLRF controller of the station [47].

The simulation allows to set and test different algorithms of identification and reduced models to define the analytic transfer function of the RF stations before the final commissioning of the tool. Results from these test are depicted in Figure 6.1, where the transfer functions of the klystron and cavity are estimated and the corresponding analytical model is fitted.

The identification and configuration tools were used for the January 2010 startup. With these tools, not only the commissioning time for the RF stations was reduced from multiple days to a few hours, but also the RF stations were commissioned remotely and robustly. The tools were also expanded to be study noise mechanisms in Chapters 7 and 8. They can also be used to study future high current operations, estimate performance margins, and define optimal operation points.

\subsection{LHC, PEP-II LLRF Similarities}

The LHC and PEP-II RF systems follow the same fundamental architecture. Detailed descriptions of the systems have been presented for LHC [59] and PEP-II [7]. Both systems employ feedback techniques to regulate the cavity voltage and phase, to 

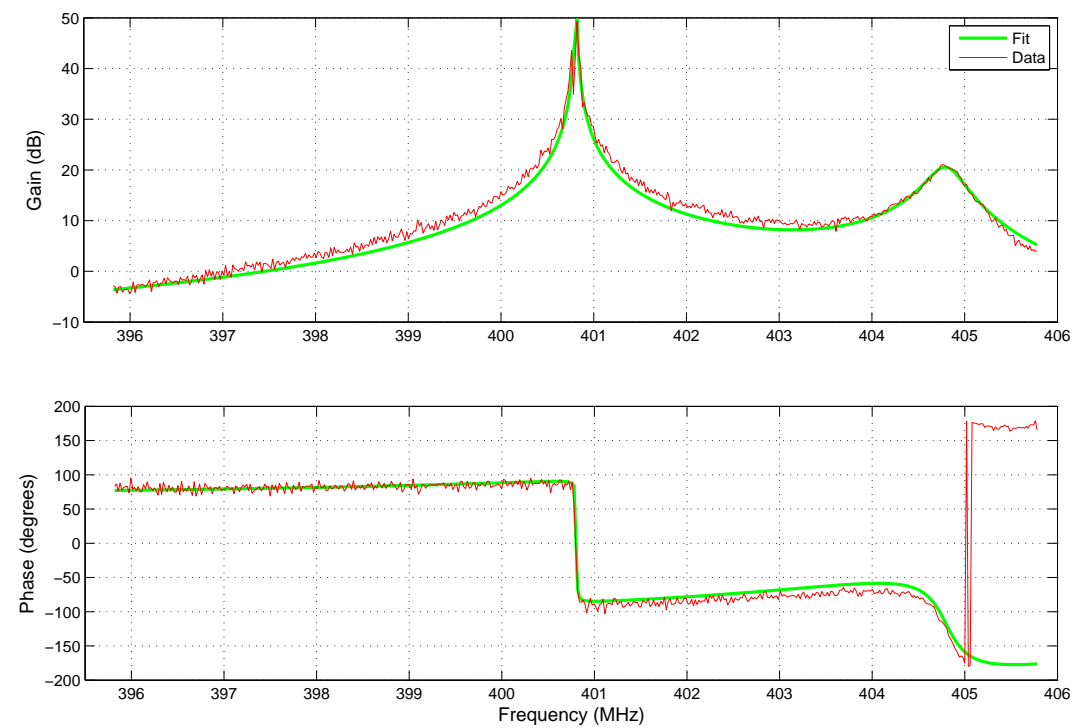

Figure 6.1: RF station transfer function based on simulated data and fitted linear model.

reduce the impedance seen by the beam, and to increase the beam stability.

Even though the critical issues are different for the two facilities, in both cases they result from the interaction between the longitudinal beam dynamics and the RF system. This fact allows us to use our simulation model and our experience from the PEP-II operations and analysis, as a basis for the LHC studies. In both cases we model the subsystems that act in a fast time scale and affect the RF station-beam interaction.

\subsubsection{System Description}

The LHC RF system consists of 8 RF stations per beam. The RF system accelerates the beam during the ramp, compensates the small energy losses during coasting, and also provides longitudinal focusing. The beam and the RF station are two dynamic systems with a strong interaction, which complicates stability considerations for the composite system. A simplified block diagram of the LHC RF system is shown in Figure 6.2. 


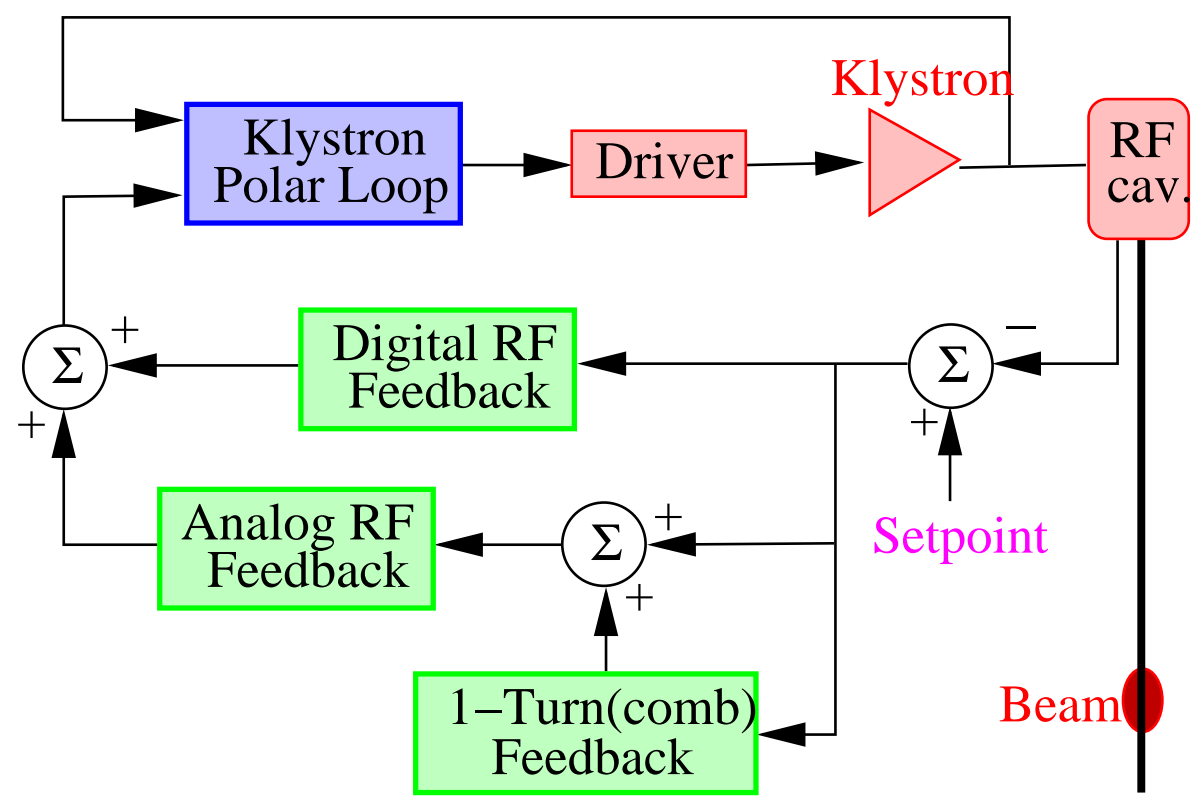

Figure 6.2: Simplified LHC RF block diagram.

Each RF station includes an accelerating super-conducting cavity, a $330 \mathrm{~kW}$ klystron, and the LLRF system consisting of the klystron polar loop and the impedance control feedback system. Figure 6.3 shows the LHC RF Cavern including the superconducting cavities, klystrons, and LLRF ${ }^{1}$. The super-conducting cavity has an $R / Q$ of 45 , a resonance frequency of $400.8 \mathrm{MHz}$, and a mechanical cavity tuner with a range from -100 to $0 \mathrm{kHz}$. The cavity voltage and loaded quality factor $Q_{L}$ are set to $1 \mathrm{MV}$ and 20,000 respectively during injection and to $2 \mathrm{MV}$ and 60,000 during physics, for nominal intensity beams. An LHC super-conducting cavity is shown in Figure 6.4. The klystrons used at LHC are inherently non-linear. The klystron polar loop used at the LHC acts around the klystron to reject power supply perturbations and compensate the gain and phase shift of the non-linear klystron at low frequencies for different operation points. The feedback system controls the accelerating fundamental impedance of the RF station to achieve longitudinal stability. It incorporates digital and analog paths, as well as the 1-Turn feedback (comb), which acts to reduce the impedance at the synchrotron sidebands. The LLRF crates are shown in

\footnotetext{
${ }^{1}$ Figures $6.3,6.4$, and 6.5 are LHC pictures by Dan Van Winkle
} 


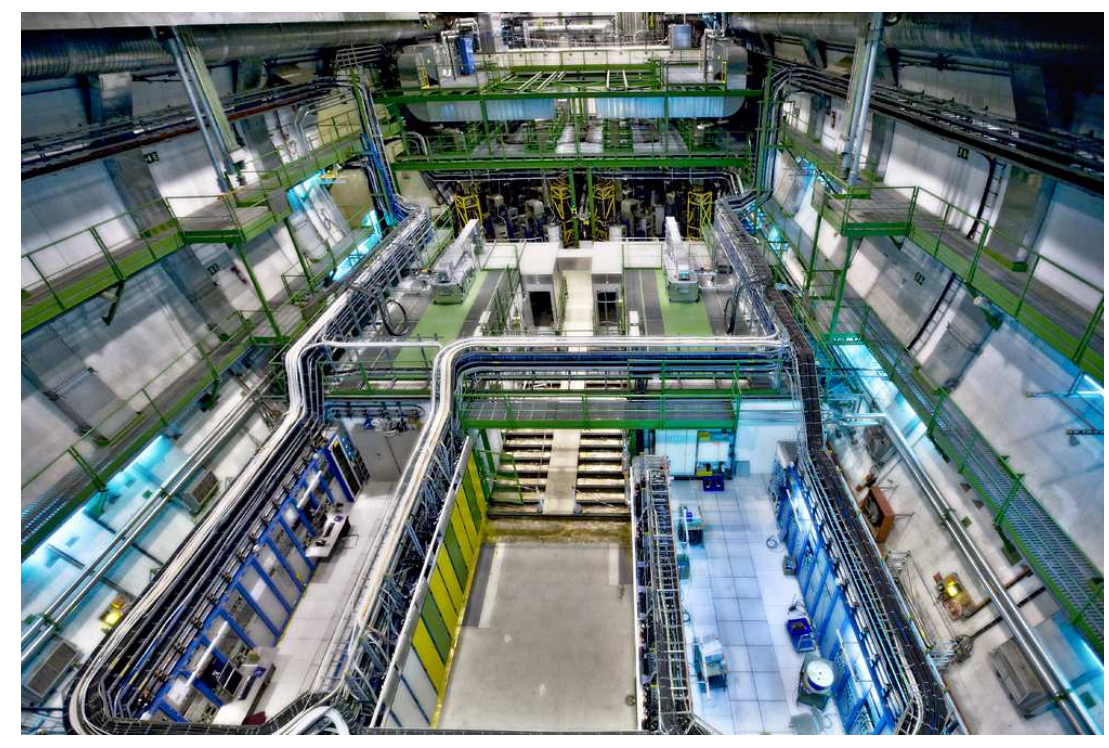

Figure 6.3: RF Cavern.

Figure 6.5.

By comparison to Figure 4.1, the RF station block diagrams for the two machines show great similarities. The actual implementations are different, but the topology is exactly the same. The PEP-II LLRF includes the Direct Loop, a lead-lag compensation in analog, whereas the LHC LLRF includes just a lag compensation composed of both a Digital and an Analog path. Both LLRF systems include 1-Turn Feedback (comb) compensation. The reason for the close resemblance is that both systems are based on the early work of D. Boussard and F. Pedersen. A detailed block diagram and description of the LHC LLRF components can be found in [59].

\subsection{Simulation and Model Description}

The components modeled in our simulation model include

- the accelerating super-conducting cavity with an $R / Q$ of 45 and a resonance frequency of $400.8 \mathrm{MHz}$,

- the $300 \mathrm{~kW}$ klystron, 


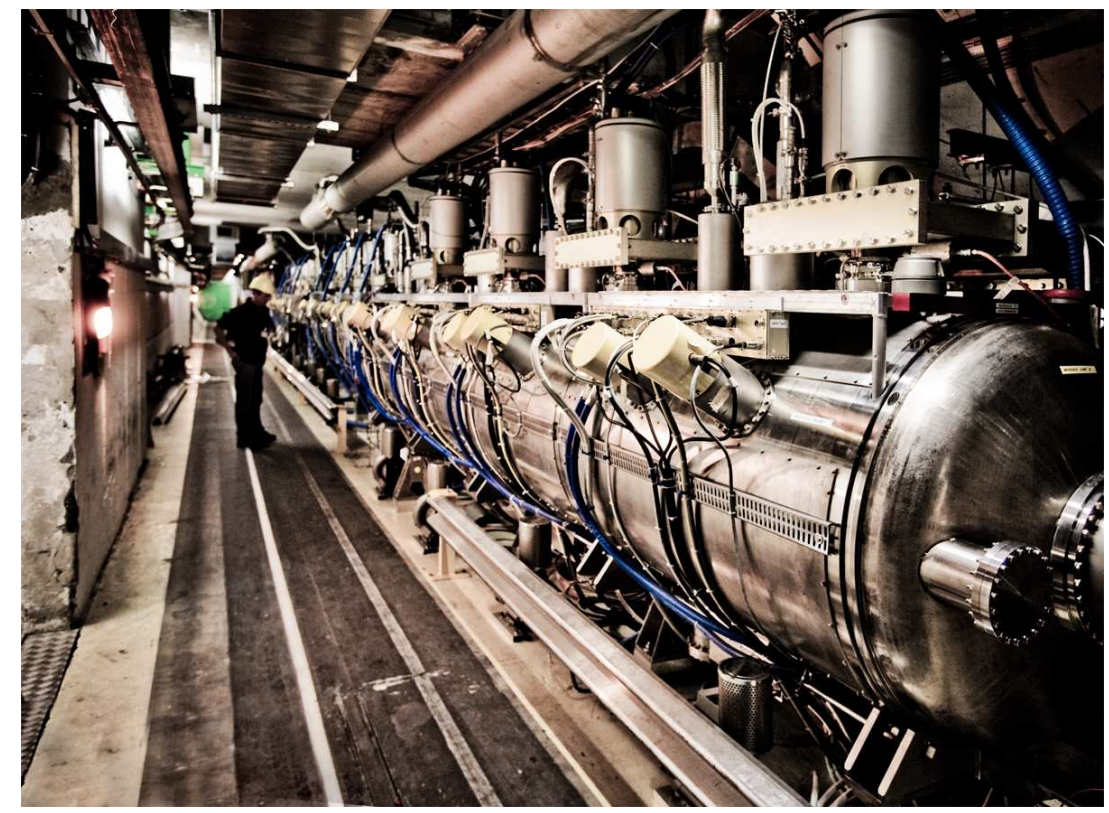

Figure 6.4: LHC super-conducting cavity.

- the klystron polar loop,

- the impedance controlling feedback (both digital and analog paths), including quantizing effects in the digital 1-turn feedback system

- cable and processing delays.

- and the beam.

To accurately describe the system, reduced models of the individual components are included in the simulation. The waveguide, cable, and processing delays are included, and the gains and phases of the RF feedback components are adjusted in a similar manner as for the real machine. The 1-Turn feedback (comb), which acts to reduce the impedance at the synchrotron sidebands, has not yet reached the hardware commissioning phase, but will be validated when data is available during commissioning.

The simulation is developed as a block system in Simulink. The slow loop dynamics (software control regulators) are sufficiently slow compared to the time scale of the simulation that they are calculated as initial conditions (constants) in Matlab. 


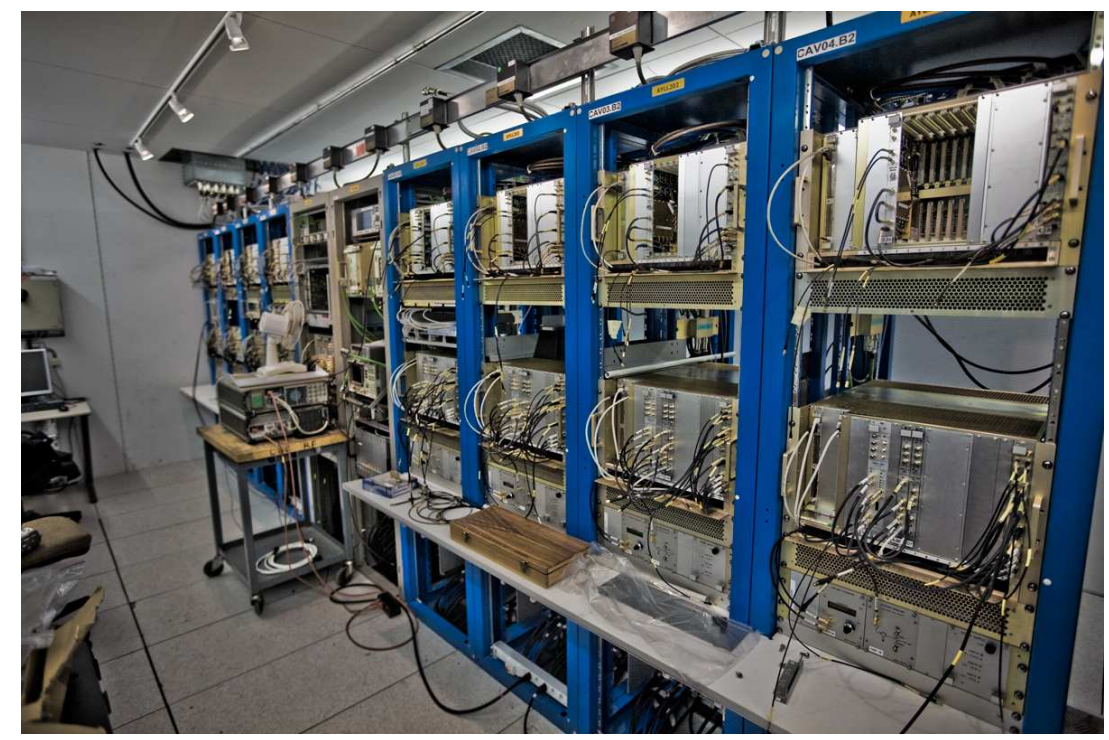

Figure 6.5: LHC LLRF crates.

An earlier simulation effort by J. Holma [60] had been used to study and set-up the loops on a full-scale test bunch that included the LLRF, klystron, and cavity. This initial model did not include the beam dynamics, but certain components were incorporated in this work.

\subsection{Simulation Validation}

For the initial simulation validation, transfer function measurements of the LHC RF stations during the RF hardware commissioning were used. After this initial validation, the simulation was used to predict diffusion effects and multi-bunch stability limits for the LHC in Chapter 7. The simulation was further validated by comparing some of these predictions with measurements from the machine in Chapter 8.

In Figures 6.6 and 6.7 the klystron transfer functions from the measurements and the simulation are presented, as measured from the input of the Driver to the output of the Klystron as shown in the block diagram (Figure 6.2). The klystron transfer function exhibits a secondary resonance at $404.8 \mathrm{MHz}$ with a $Q$ of 1100 . The simulation shows these characteristics with good agreement in amplitude. The 
phase discrepancy between the plots is due to delay calibration offsets included in the network analyzer used for the LHC measurements.

The superconducting cavity and the klystron polar loop are then measured in the real machine and the simulation. The resulting transfer functions from the input of the klystron polar loop to the output of the cavity can be seen in Figure 6.8 showing once again good agreement. The effect of the system delay is obvious on the phase plot.

Finally, Figure 6.9 shows the transfer function of the RF station in closed loop, with the RF feedback loop regulating the system. The RF feedback's amplitude and phase are adjustable in the simulation, as in the real machine.

As can be seen from these figures, there is a close agreement between the simulation and the measurements of the real RF system at LHC. 

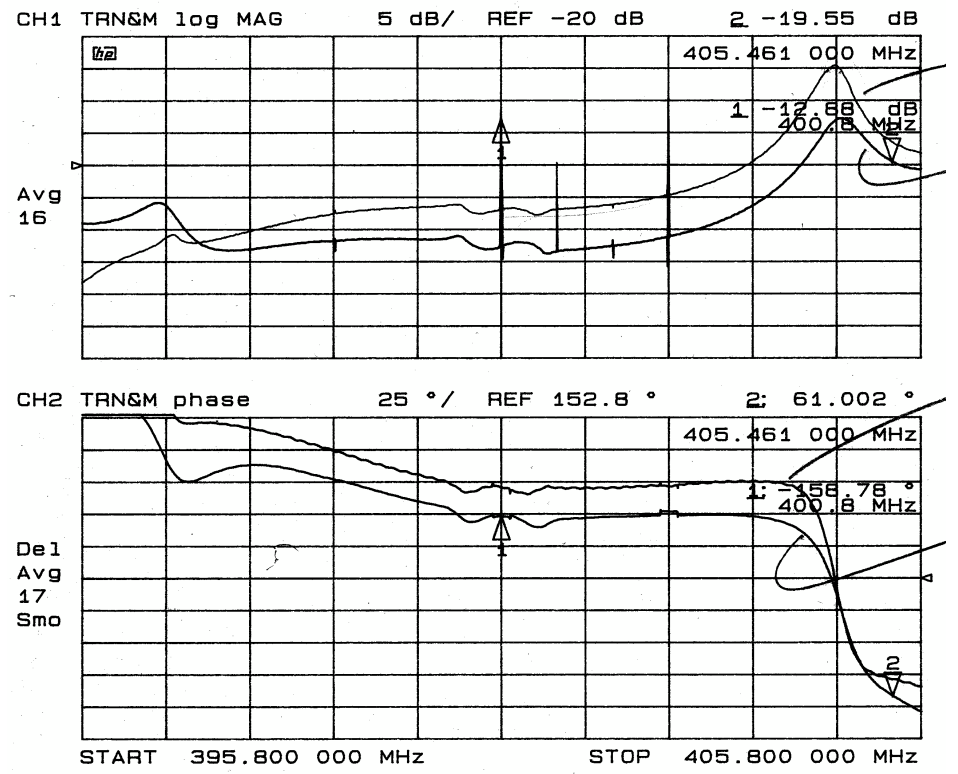

Figure 6.6: LHC Measured Klystron Transfer Function
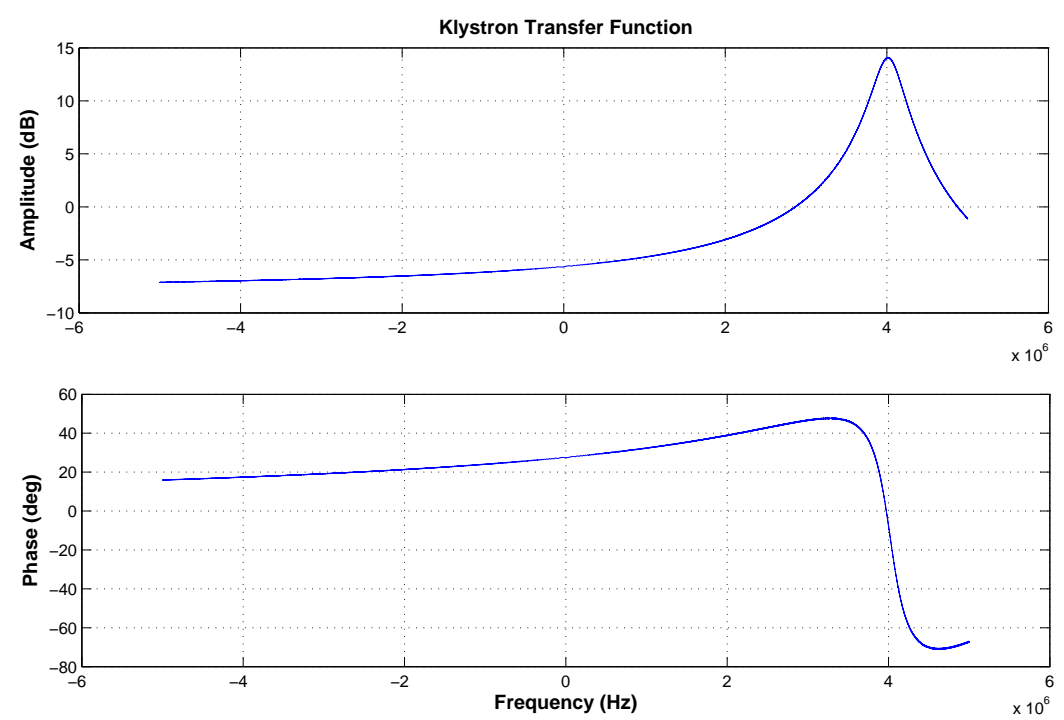

Figure 6.7: SLAC Simulated Klystron Transfer Function. 

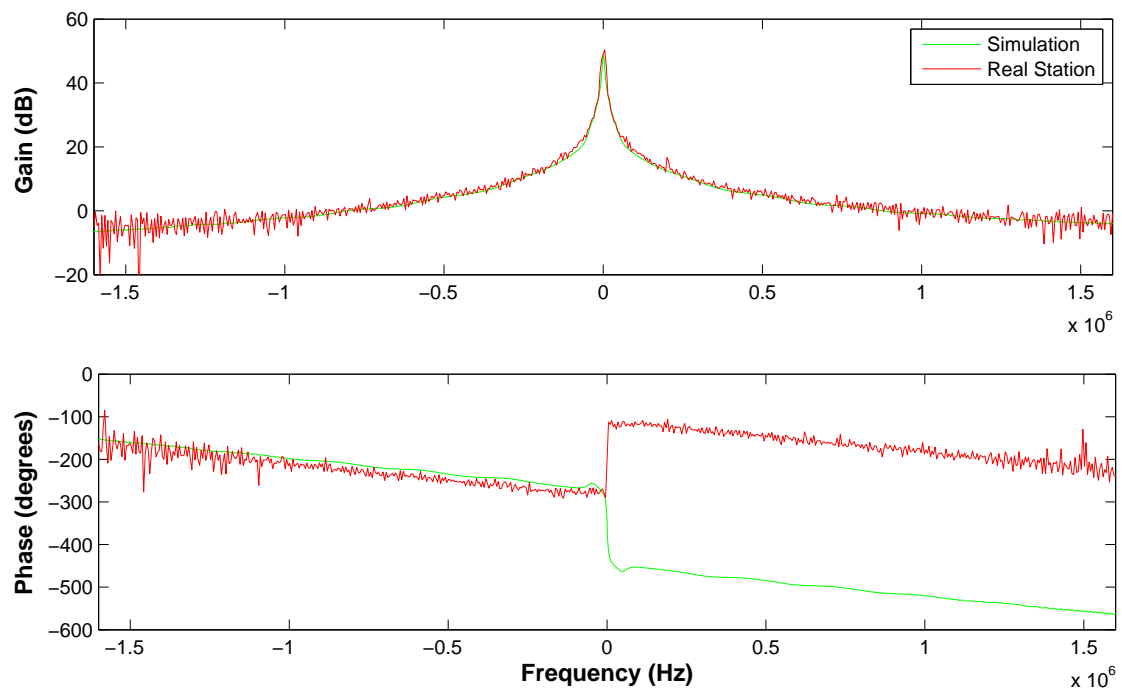

Figure 6.8: LHC Measured and Simulated Open Loop RF station Transfer Function.
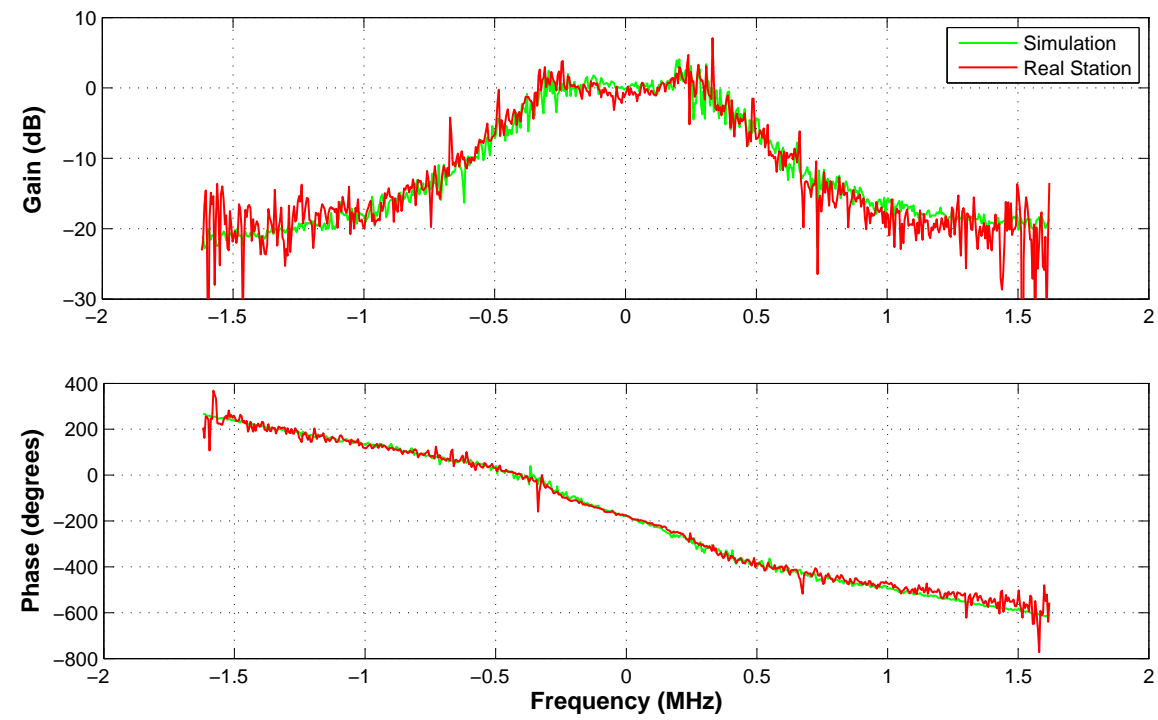

Figure 6.9: LHC Measured and Simulated Closed Loop RF station Transfer Function. 


\section{Chapter 7}

\section{LHC Longitudinal Beam Dynamics Studies}

The LHC RF station-beam interaction strongly influences the longitudinal beam dynamics, both single bunch and collective effects. Non-linearities and noise generated within the RF accelerating system interact with the beam and contribute to beam motion and longitudinal emittance blowup. Thus, the noise power spectrum of the RF accelerating voltage strongly affects the longitudinal beam distribution. Furthermore, the coupled-bunch instabilities are also directly affected by the RF components and the configuration of the LLRF feedback loops. A formalism relating the longitudinal beam dynamics with the RF system configurations, an estimation of collective effects stability margins, and an evaluation of longitudinal sensitivity to various LLRF parameters and configurations are presented in this Chapter.

The new directions for the LHC longitudinal studies with respect to PEP-II are presented in Section 7.1. Section 7.2 outlines the major noise sources of the system and defines their relationship with the RF accelerating voltage noise. In Section 7.3 a quantitative description of the relationship between the noise spectral density and the longitudinal beam emittance will be presented, as a function of the RF loop configuration and the system noise. With this formalism and the simplification from Section 7.2, it is then possible to study the single bunch dynamics for RF configurations of interest, as shown in Section 7.4. The stability criterion for coupled-bunch 
instabilities, the estimated growth rates, and the sensitivity of multi-bunch dynamics on the RF parameters are discussed in Section 7.5.

\subsection{New Directions for LHC}

Even though the machine architecture is very similar between PEP-II and LHC, the performance limiting longitudinal beam dynamics are quite different. Due to the heavy beam loading at PEP-II, the coupled-bunch instabilities required careful tuning of the LLRF system and dedicated bunch-by-bunch feedback systems. At LHC the reduced beam loading together with the substantial Landau damping due to the long bunch length makes the multi-bunch effects less critical. On the other hand, synchrotron damping is low in a hadron machine, so that noise and imperfections in the LLRF systems can contribute to growth in longitudinal emittance and reduction in beam life time.

During a long store, the relationship between the energy lost to synchrotron radiation and the noise injected to the beam by the RF accelerating voltage determines the growth of the bunch energy spread and longitudinal emittance. Since the proton synchrotron radiation in the LHC is very low, the beam diffusion is extremely sensitive to RF perturbations.

Single-bunch longitudinal emittance growth as well as beam stability related to collective effects are examined in this Chapter. Both of these longitudinal dynamics effects are strongly coupled to the effective impedance of the RF station and the configurations of the feedback loop. The RF configuration is defined by the design choices of components and signal levels, as well as the operational choices of variable parameters. Different approaches on the component and parameter selection can have a significant effect on the stability and characteristics of the beam.

For these studies, the LHC LLRF system has been modeled with the existing technology implementation. The effect of the operational choices on controller settings is then investigated. The objective of this work is to verify high-current and upgraded operating conditions of the LHC, study optimal configuration techniques to achieve minimum RF station effective impedance, estimate noise and stability limits of the 
system, and possible impacts of technical aspects of the implementation. An ultimate goal is to use this knowledge to build a base of future impedance-controlled RF and LLRF system techniques for future and upgrades of existing machines.

\subsection{Noise Sources}

The single bunch longitudinal emittance growth greatly depends on the noise level in the RF accelerating voltage. The major noise sources in the RF system include components in the LLRF boards, the RF reference (local oscillator), the klystron driver amplifier, the klystron power supply, low frequency sources (microphonics, ground vibrations, cooling system etc.), the effect of the non-uniform beam current on the RF cavity voltage, cross-talk between channels, and more. The spectrum of these sources spans from very low to very high frequencies.

The models presented in this Chapter work for both low frequency and wideband sources. Initial measurements suggest that the LLRF noise contributions are indeed wideband, but that there are also significant contributions from the RF reference (local oscillator) at low frequencies. The quantitative results presented in this Chapter assume wideband sources for simplicity, in particular the LLRF noise and the klystron driver amplifier, as shown in Figure 7.1. This choice is based on the relative noise power of the components in the LHC implementation. The LLRF noise includes several contributions such as the digital quantizing noise and arithmetic noise in digital signal processing, thermal noise, analog/digital demodulator, and modulator. Based on an understanding of the engineering implementation of the system, these sources can be considered broadband and incoherent.

Imperfections in the technical implementation - such as the channel crosstalk are not included in this model. If these effects were determined to be essential though, they could be easily added in the model. Similarly, the system sensitivity to these effects could be studied with the simulation and a threshold amount for each such technical imperfection could be estimated.

It should be noted that the klystron power supplies introduce coherent noise at the $50 \mathrm{~Hz}$ harmonics in all the stations. The synchrotron frequency crosses the $50 \mathrm{~Hz}$ line 


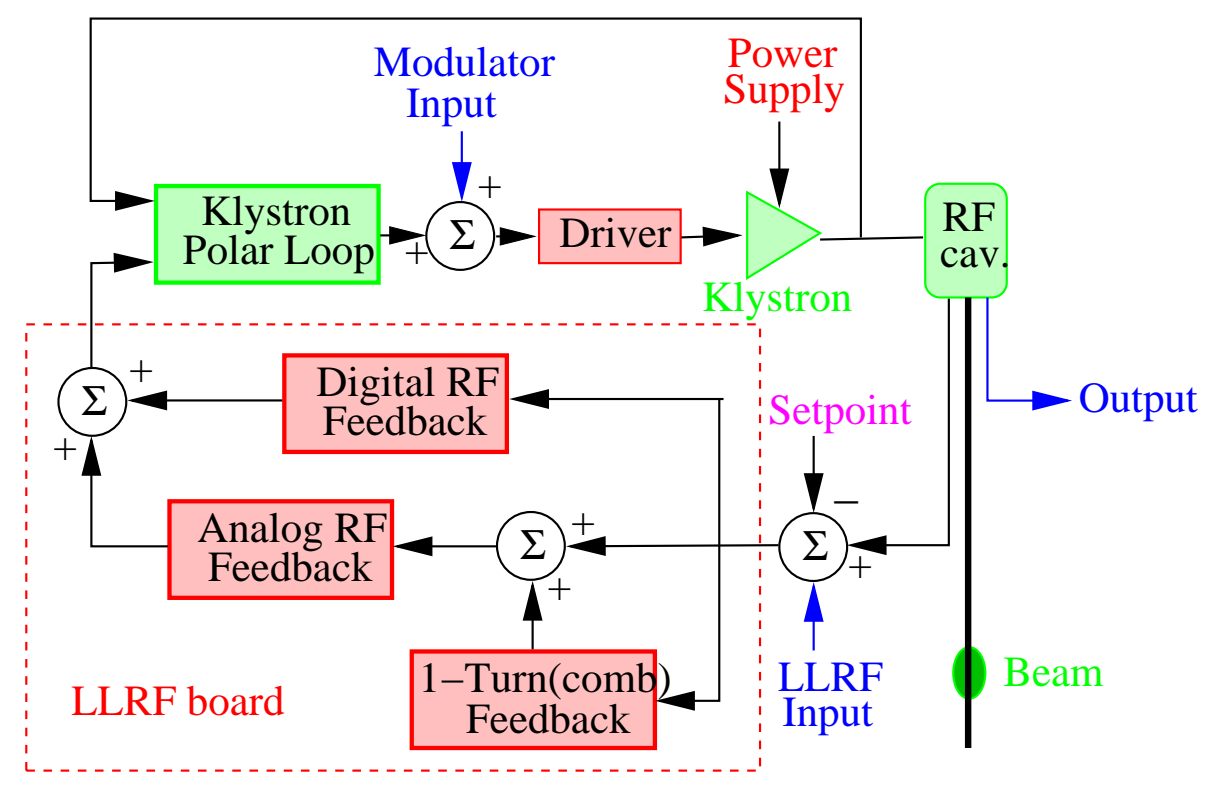

Figure 7.1: (Color) Noise Sources. Blocks in red represent the major noise sources, in blue are the equivalent noise sources for simulations purposes, and the other components are shown in green.

during the ramp, which can lead to a resonant effect [61]. The longitudinal emittance growth formalism presented in this work does not include this phenomenon.

The individual noise sources for the LLRF components are distributed throughout the electronics of the system. To be able to effectively study the various contributions, it is helpful to concentrate them in two equivalent noise sources located either at the input of the LLRF board, or the output of the klystron polar loop (the input of the modulator), as shown in Figure 7.1. Both of these sources are in baseband and can be modeled as two independent sources in the in-phase and quadrature channels, for a total of four noise sources.

The accelerating voltage noise is modeled in amplitude $a_{c a v}(t)$ and phase $\phi_{\text {cav }}(t)$. To calculate the relationship between an input vector perturbation at the $m^{\text {th }}$ source

$$
n^{m}(t)=\left(\begin{array}{l}
n_{i}^{m} \\
n_{q}^{m}
\end{array}\right)
$$

and the amplitude or phase of the cavity voltage we linearize the response of the 
RF station around the operation point and determine the impulse response $h^{m}(t)$ between the output and the input

$$
\begin{aligned}
\left(\begin{array}{l}
a_{c a v}^{m}(t) \\
\phi_{c a v}^{m}(t)
\end{array}\right) & =\left[\begin{array}{ll}
h_{a i}^{m} & h_{a q}^{m} \\
h_{\phi i}^{m} & h_{\phi q}^{m}
\end{array}\right] *\left(\begin{array}{c}
n_{i}^{m} \\
n_{q}^{m}
\end{array}\right)(t) \\
& =\left(\begin{array}{c}
h_{a}^{m} \\
h_{\phi}^{m}
\end{array}\right) * n^{m}(t)
\end{aligned}
$$

where $h_{a}^{m}=\left[h_{a i}^{m} h_{a q}^{m}\right]$ and $h_{\phi}^{m}=\left[h_{\phi i}^{m} h_{\phi q}^{m}\right]$.

Since the system is linear, we can use superposition to get

$$
\left(\begin{array}{c}
a_{c a v}(t) \\
\phi_{c a v}(t)
\end{array}\right)=\sum_{m}\left(\begin{array}{c}
h_{a}^{m} \\
h_{\phi}^{m}
\end{array}\right) * n^{m}(t)
$$

where the summation is over all the noise sources. The impulse responses $h^{m}(t)$ depend on the operational configuration of the RF station.

\subsection{Formalism for Bunch Length Estimation}

During a long store, the bunch energy spread and longitudinal emittance shrink due to the small synchrotron radiation damping, whereas any noise injected in the accelerating RF voltage leads to longitudinal emittance blowup. Optimally, these two mechanisms should balance and the beam should keep a constant bunch length. In this Section, a formalism relating the bunch emittance growth with the noise in the accelerating voltage is presented. The accelerating voltage depends strongly on the configurations of the RF station and the LLRF feedback systems.

Following [1] and Section 2.1.1 it can be shown that the longitudinal equations of beam motion are

$$
\begin{aligned}
\dot{\phi} & =\frac{\eta \omega_{R F}}{E_{o}} \epsilon \\
\dot{\epsilon} & =\frac{1}{T_{o}}\left[q V_{o} \sin \left(\phi_{s}+\phi\right)-U_{\text {rad }}\left(\epsilon+E_{o}\right)\right]
\end{aligned}
$$


where $\phi, \epsilon$ are the phase and energy of the particles with respect to the synchronous particle, the RF voltage is $V=V_{o} \sin \left(\phi_{s}+\phi\right), \eta$ is the slip factor, $E_{o}$ the beam energy, $T_{o}$ the revolution period, $q$ the charge of a proton, $\omega_{R F}$ the angular RF frequency, $\phi_{s}$ the phase of the synchronous particle, and $U_{r a d}(E)$ the synchrotron radiation energy emitted by a particle of energy $E$ over a turn. It should be noted that even though this equation is defined for a single particle, it extends to the whole multiparticle bunch. This set of equations can be described as a stochastic differential equation.

The cavity amplitude modulation noise $a_{c a v}(t)$ and phase noise $\phi_{c a v}(t)$ are sampled by the beam with a period $T_{o}$ resulting in the perturbations $a(t)$ and $\tilde{\phi}(t)$. In the presence of these perturbations, the beam motion Equation 7.2 becomes

$$
\begin{aligned}
\dot{\phi}= & \frac{\eta \omega_{R F}}{E_{o}} \epsilon \\
\dot{\epsilon}= & \frac{1}{T_{o}}\left[q V_{o}(1+a(t)) \sin \left(\phi_{s}+\phi-\tilde{\phi}(t)\right)-\right. \\
& \left.U_{\text {rad }}\left(\epsilon+E_{o}\right)\right] \\
= & \frac{1}{T_{o}}\left[q V _ { o } ( 1 + a ( t ) ) \left(\sin \left(\phi_{s}\right) \cos (\phi-\tilde{\phi}(t))+\right.\right. \\
& \left.\left.\cos \left(\phi_{s}\right) \sin (\phi-\tilde{\phi}(t))\right)-U_{\text {rad }}\left(\epsilon+E_{o}\right)\right]
\end{aligned}
$$

For small energy oscillations, $\epsilon$ and $\phi$ are close to zero, so it is possible to linearize around the synchronous particle coordinates. Then, Equation 7.3 becomes

$$
\begin{aligned}
\dot{\epsilon}= & \frac{1}{T_{o}}\left[q V_{o}\left(\sin \left(\phi_{s}\right)+(\phi-\tilde{\phi}(t)) \cos \left(\phi_{s}\right)+a(t) \sin \left(\phi_{s}\right)\right)\right. \\
& \left.-U_{o}-\epsilon D\right] \\
= & \frac{1}{T_{o}}\left[q V_{o}\left((\phi-\tilde{\phi}(t)) \cos \left(\phi_{s}\right)+a(t) \sin \left(\phi_{s}\right)\right)-\epsilon D\right]
\end{aligned}
$$

where $D \approx 2 U_{o} / E_{o}$ with $U_{o}$ the synchrotron radiation of the synchronous particle, and $q V_{o} \sin \left(\phi_{s}\right)=U_{o}$. The second order perturbation term $a(t)(\phi-\tilde{\phi}(t)) \cos \left(\phi_{s}\right) \approx 0$ has been dropped.

It is obvious from Equation 7.4 that the phase noise is much more significant than 
the amplitude noise, since $\phi_{s}$ is close to $180^{\circ}$ in hadron machines, so that

$$
\left.(\phi-\tilde{\phi}(t)) \cos \left(\phi_{s}\right) \gg a(t) \sin \left(\phi_{s}\right)\right) .
$$

Therefore, using Equations 7.2, 7.4, and assuming that the amplitude noise is negligible, we get the linearized longitudinal equations of motion:

$$
\begin{aligned}
\dot{\phi} & =\frac{\eta \omega_{R F}}{E_{o}} \epsilon \\
\dot{\epsilon} & =\frac{q \dot{V}\left(\phi_{s}\right)}{\omega_{R F} T_{o}}(\phi-\tilde{\phi}(t))-\frac{D}{T_{o}} \epsilon
\end{aligned}
$$

where $\dot{V}\left(\phi_{s}\right)=\omega_{R F} V_{o} \cos \left(\phi_{s}\right)$ is the RF gradient for the synchronous particle.

The particle beam samples the cavity phase noise $\phi_{c a v}(t)$ every revolution harmonic, so that

$$
\begin{aligned}
\tilde{\phi}(t) & =\sum_{k=-\infty}^{\infty} \delta\left(t-k T_{o}\right) \phi_{\text {cav }}(t) \\
& =\sum_{k=-\infty}^{\infty} \delta\left(t-k T_{o}\right) \sum_{m} h_{\phi}^{m} * n^{m}(t)
\end{aligned}
$$

according to Equation 7.1. To simplify the notation, and since the noise sources are uncorrelated, the analysis is carried using a generic representation for one of the terms in the summation over $m$, so that

$$
\tilde{\phi}(t)=\sum_{k=-\infty}^{\infty} \delta\left(t-k T_{o}\right)\left(h_{g} * n_{g}\right)(t)
$$

where $\left(h_{g}, n_{g}\right)$ could be either $\left(h_{\phi i}, n_{i}\right)$ or $\left(h_{\phi_{q}}, n_{q}\right)$. The approximation of the sampling by an impulse is still valid for the ensemble of equations of all particles in a bunch, since the 1 ns bunch is very fast compared to the period of the loop dynamics (approximately $3 \mu \mathrm{s}$, since the bandwidth of $\phi_{c a v}$ is roughly $300 \mathrm{kHz}$ ).

To simplify the equations of motion, $\epsilon$ is transformed to a normalized momentum 
$p$,

$$
p=\frac{\eta \omega_{R F}}{E_{o}} \epsilon
$$

so that Equation 7.5 becomes

$$
\begin{aligned}
\dot{\phi} & =p \\
\dot{p} & =-\omega_{s}^{2} \phi-2 \alpha p+\omega_{s}^{2} \tilde{\phi}(t)
\end{aligned}
$$

where

$$
\begin{aligned}
\omega_{s} & =-\sqrt{\frac{\eta q \dot{V}(0)}{E_{o} T_{o}}} \\
\alpha & =\frac{D}{2 T_{o}} .
\end{aligned}
$$

The vector

$$
X=\left(\begin{array}{l}
\phi \\
p
\end{array}\right)
$$

is defined, so that Equation 7.6 can be rewritten as

$$
d X=A X(t) d t+d v
$$

where

$$
\begin{aligned}
A & =\left[\begin{array}{cc}
0 & 1 \\
-\omega_{s}^{2} & -2 \alpha
\end{array}\right] \\
\frac{d v}{d t} & =\left[\begin{array}{c}
0 \\
\omega_{s}^{2}
\end{array}\right] \tilde{\phi}(t)=K \tilde{\phi}(t)
\end{aligned}
$$

To estimate the bunch length $\sigma_{z}$, it is necessary to determine the second order moment of $\phi$, since

$$
\sigma_{z}=\frac{c}{\omega_{R F}} \sigma_{\phi}
$$

Following the procedure outlined in [62] and [63], the autocorrelations of the perturbation $R_{\tilde{\phi} \tilde{\phi}}$ and output $R_{x x}$, as well as their crosscorrelation $R_{\tilde{\phi} x}$ are determined. It 
should be noted that $R_{\tilde{\phi} \tilde{\phi}}$ and $R_{\tilde{\phi} x}$ are wide sense stationary.

$$
\begin{aligned}
R_{\tilde{\phi} \tilde{\phi}}\left(t_{2}-t_{1}\right) & =E\left[\tilde{\phi}\left(t_{2}\right), \tilde{\phi}\left(t_{1}\right)\right] \\
= & \left.\left(\sum_{k=-\infty}^{\infty} \delta\left(t_{2}-k T_{o}\right) h_{g}\left(t_{2}\right)\right) * E\left[n_{g}\left(t_{2}\right) n_{g}\left(t_{1}\right)\right] *\left(\sum_{k=-\infty}^{\infty} \delta\left(t_{1}-k T_{o}\right) h_{g}\left(t_{1}\right)\right)\right) \\
R_{\tilde{\phi} x}\left(t_{2}-t_{1}\right) & =\int_{-\infty}^{t_{1}} E\left[\tilde{\phi}\left(t_{2}\right) \tilde{\phi}\left(t_{1}-\theta\right)\right]\left(e^{A \theta}\left[\begin{array}{c}
0 \\
\omega_{s}^{2}
\end{array}\right]\right)^{T} d \theta \\
& =\int_{-\infty}^{t_{1}} R_{\tilde{\phi} \tilde{\phi}}\left(t_{2}-t_{1}+\theta\right)\left(e^{A \theta}\left[\begin{array}{c}
0 \\
\omega_{s}^{2}
\end{array}\right]\right)^{T} d \theta \\
& =\int_{-\infty}^{t_{1}} R_{\tilde{\phi} \tilde{\phi}}\left(t_{2}-t_{1}-\theta\right)\left(e^{-A \theta}\left[\begin{array}{c}
0 \\
\omega_{s}^{2}
\end{array}\right]\right)^{T} d \theta \\
R_{x x}\left(t_{2}, t_{1}\right) & =\left[\begin{array}{c}
\sigma_{\phi}^{2}\left(t_{2}, t_{1}\right) \\
E\left[p\left(t_{2}\right) \phi\left(t_{1}\right)\right] \\
\sigma_{p}^{2}\left(t_{2}, t_{1}\right)
\end{array}\right] \\
& =\int_{-\infty}^{t_{2}} e^{A \theta}\left[\begin{array}{c}
0 \\
\omega_{s}^{2}
\end{array}\right] E\left[\tilde{\phi}\left(t_{2}-\theta\right) x^{T}\left(t_{1}\right)\right] d \theta \\
& =\int_{-\infty}^{t_{2}} e^{A \theta}\left[\begin{array}{c}
0 \\
\omega_{s}^{2}
\end{array}\right] R_{\tilde{\phi} x}\left(t_{2}-t_{1}-\theta\right) d \theta
\end{aligned}
$$

Since the system is linear and stable, the expressions in Equation 7.7 converge to equilibrium values defined by the noise power and synchrotron radiation damping. These equilibrium values can be estimated by setting $\tau=t_{2}-t_{1}$ and then taking the limit of Equation 7.7 as $t_{1}, t_{2} \rightarrow \infty$, since $R_{x x}$ is asymptotically wide sense stationary, to get

$$
R_{x x}(\tau)=e^{A \tau} *\left[\begin{array}{cc}
0 & 0 \\
0 & \omega_{s}^{4}
\end{array}\right] R_{\tilde{\phi} \tilde{\phi}}(\tau) * e^{-A^{T} \tau}
$$

which gives the correlation matrix due to the noise perturbation filtered by both the RF station and the beam dynamics. By applying the Fourier Transform to $R_{x x}(\tau)$ 
from Equation 7.8 and substituting for the noise autocorrelation $R_{\tilde{\phi} \tilde{\phi}}(\tau)$ from Equation 7.7 , the power spectral density $S_{g}(f)$ of $X(t)$ due to the generic term is obtained

$$
S_{g}(f)=B_{g} S_{N_{g}}(f) B_{g}^{H},
$$

where $S_{N_{g}}(f)=\mathcal{F}\left(E\left[n_{g}\left(t_{1}\right) n_{g}\left(t_{2}\right)\right]\right)$, the superscript $H$ denotes transpose complex conjugate, and $B_{g}$ is given by

$$
\begin{aligned}
B_{g} & =(2 \pi j f I-A)^{-1}\left[\begin{array}{cc}
0 & 0 \\
0 & \omega_{s}^{2}
\end{array}\right]\left(\sum_{k=-\infty}^{\infty} \delta\left(f-k f_{\text {rev }}\right)\right) * H_{g}(f) \\
& =(2 \pi j f I-A)^{-1}\left[\begin{array}{cc}
0 & 0 \\
0 & \omega_{s}^{2}
\end{array}\right] \sum_{k=-\infty}^{\infty} H_{g}\left(f-k f_{\text {rev }}\right),
\end{aligned}
$$

where $f_{\text {rev }}$ is the beam's revolution frequency, $H_{g}(f)=\mathcal{F}\left(h_{g}(t)\right)$ is the frequency response of the RF station for a particular source and channel, and $(2 \pi j f I-A)^{-1}$ is a matrix transfer function characterizing the beam filtering of the noise spectrum.

Extending the analysis to all noise sources and channels, the total spectral density $S_{x}(f)$ is given by

$$
S_{x}(f)=\sum_{m}\left(B_{i}^{m} S_{N_{i}}^{m}(f)\left(B_{i}^{m}\right)^{H}+B_{q}^{m} S_{N_{q}}^{m}(f)\left(B_{q}^{m}\right)^{H}\right)
$$

Then, the square of the equilibrium bunch length $\sigma_{z}^{2}$ is given by

$$
\begin{aligned}
\sigma_{z}^{2} & =\frac{c^{2}}{\omega_{R F}^{2}} \sigma_{\phi}^{2} \\
& =2 \frac{c^{2}}{\omega_{R F}^{2}}\left[\begin{array}{ll}
1 & 0 \\
0 & 0
\end{array}\right] \int_{0}^{\infty} S_{x}(f) d f
\end{aligned}
$$

since the autocorrelation $R_{x x}(\tau)$ is an even function.

It is obvious from Equations 7.10 and 7.11 that the bunch length depends on the noise power spectrum injected, filtered by the corresponding RF station and beam transfer functions as intuitively expected. The aliasing effect of the periodic 
sampling of the accelerating voltage signal can also be seen. This aliasing effect practically folds the bandwidth of the closed loop RF station response $(\approx 300 \mathrm{kHz})$ on the band between DC and $f_{\text {rev }}=11.245 \mathrm{kHz}$. The aliasing greatly enhances the effect of the noise on the beam dynamics and multiplies the noise power spectrum by almost a factor of thirty. From this analysis it also follows that the aliased and loop shaped noise power spectral density at the synchrotron frequency $f_{s}$ is critical for the determination of the equilibrium bunch length, as seen from the beam transfer

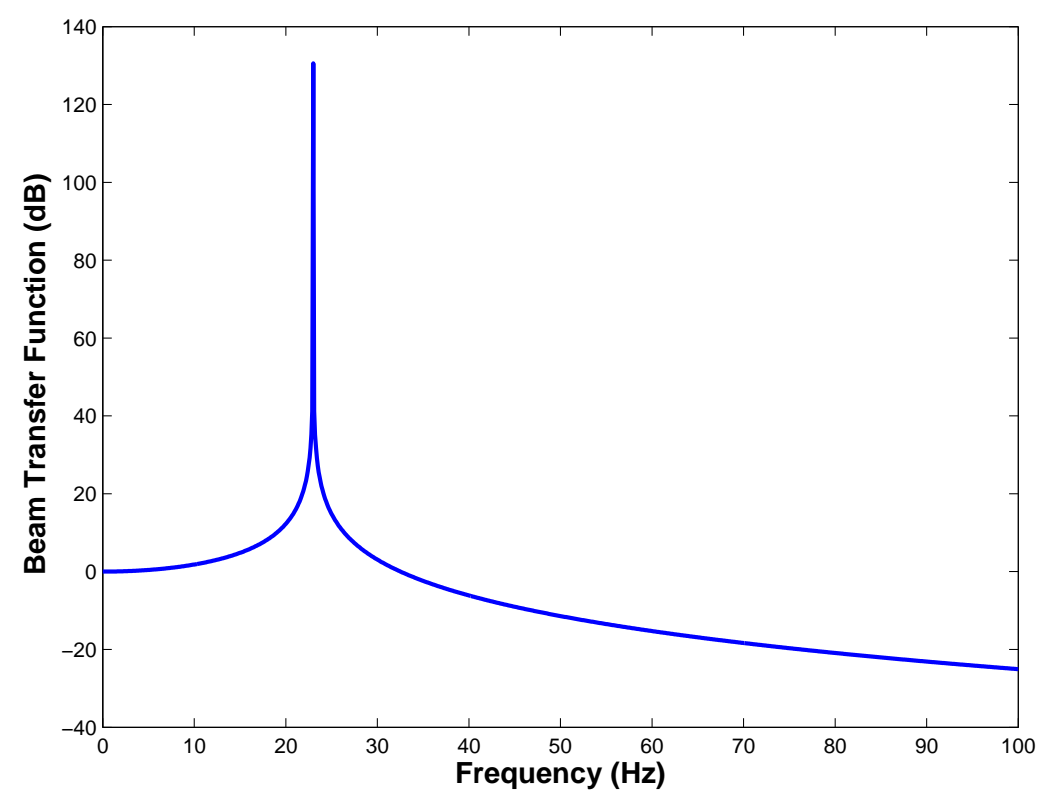

Figure 7.2: (Color) Beam Transfer Function during physics defined by $\left[\begin{array}{ll}1 & 0\end{array}\right](2 \pi j f I-$ $A)^{-1}\left[\begin{array}{ll}0 & \omega_{s}^{2}\end{array}\right]^{T}$. The resonance is even sharper during injection (longer synchrotron radiation damping time).

function depicted in Figure 7.2, which shows the relationship between the beam phase and the sampled cavity phase.

It should be noted that with this treatment, the individual noise sources with power spectrum density $S_{N}(f)$ can be shaped or colored noise sources. This is an advantage of this formalism over a similar analysis using the Fokker-Planck equation, which cannot be extended to colored noise sources, or to white noise sources shaped by the dynamics of the RF station, as discussed in [64], [65]. 
In this treatment we considered a single RF station. It is obvious from this result that the equilibrium bunch length will depend on the total power sampled by the beam. By superposition, this will be equal to the sum of the power introduced to the beam from all the RF stations.

\subsection{An Application to Single-bunch Dynamics}

Due to the very low synchrotron radiation of the protons in the LHC and the use of klystrons as final amplifiers in the RF stations, the LHC longitudinal beam emittance greatly depends on RF station perturbations and noise. Earlier studies [66] have determined the substantial variation of the LHC bunch length, but have considered the RF system as a generator in steady state. In this work, RF dynamics are now included, as well as the aliasing effect of the beam's periodic sampling of the cavity voltage on the noise power spectrum.

The equilibrium value of the bunch length can be calculated by evaluating the integral of $S_{x}(f)$ as shown in Equation 7.11, by establishing the estimated transfer function $H_{g}(f)$ around a linearized operation point, and using the known linearized beam dynamics defined by matrix $A$ and the injected noise power spectral density $S_{N_{g}}$.

Since it is impossible to separate the contributions to the total cavity noise from the various noise sources and channels, noise is only injected in one point at a time during the simulations. For a given estimated transfer function $H_{g}(f)$ it is then possible to determine the noise power spectral density $S_{N_{g}}$ that will achieve $\sigma_{\phi}(\infty)=$ $\sigma_{\phi}(0)$, thus keeping the initial beam distribution during a store. These values are a useful metric of the total power injected to the beam for each channel and noise source. Thus, they are significant of the relative importance of all the sources. This is very helpful for the analysis of the system performance, since all of the major noise sources can be modeled by an equivalent white noise source in the bandwidth of the RF loop.

As a result, different operation points provide different noise levels at the accelerating voltage. The changes of the RF station phase noise floor level due to different 
settings of the LLRF feedback loops are studied, to determine the sensitivity of the longitudinal beam emittance on various RF parameters. With these results it should be possible to determine in the future what technical components dominate the noise level and how changes in digital quantizing choices and analog components impact the emittance growth.

\subsubsection{Transfer Function Estimation: Time-domain Simula- tion}

To determine the transfer function between the noise sources and the phase of the RF accelerating voltage around a linearized operation point, the time-domain simulation of the LHC RF station-particle beam interaction presented in Chapter 7 is used. The time-domain simulation allows a simple representation of analog and digital components, as well as the inclusion of non-linear elements. By linearizing around each operation point, it is possible to estimate the frequency domain transfer function. The close relationship between the LHC and PEP-II RF systems allows the use of previous experience and tools from PEP-II operations on the LHC studies [7], [67]. Detailed descriptions of the systems have been presented for PEP-II [7] and LHC [59].

The simulation captures the architecture, parameters, technical implementation, nonlinearities and engineering details of the LLRF and RF systems. Noise effects, quantizing effects in digital systems, and dynamic range effects could also be introduced. All components shown in Figure 6.2 are included in the simulation. Due to the computation complexity, it is only reasonable to run the simulation for the equivalent of tens of milliseconds of real machine time. It is then possible to extract beam and station parameters to study the longitudinal beam dynamics and the RF station operation. The time-domain simulation has also been used as a development environment for the LHC optimization and configuration tools [57], [58].

\subsubsection{RF Station Configurations of Interest}

Each operation point is defined by the RF station configuration; the set of values for all the adjustable loop parameters. These parameters are 1) beam parameters, such 
as the beam energy and average beam current, 2) High-Level RF station settings: the klystron operation point, the cavity voltage, detuning, and loaded quality factor $Q_{L}$, and 3) the LLRF parameters, Analog/Digital loop gain $G$, Controller phase $\phi$, and 1-Turn feedback gain and delay. The choices of values for this work is described below.

For each operation point, the beam and High-Level RF parameters are predetermined. Based on these operational conditions and the current technical implementation of the LHC RF system, the LLRF parameters are then adjusted to reduce the cavity fundamental impedance presented to the beam, while satisfying RF loop stability requirements. The optimal values are determined for each configuration, using the same LHC optimal configuration tools as in the real system [57], [58], [68].

\section{Beam Parameters}

During normal operations, each of LHC's rings is filled from the SPS with particles at an energy of $450 \mathrm{GeV}$ and then ramped to the collision energy (nominally $7 \mathrm{TeV}$ per beam). There are three interesting stages of the LHC operation: at the beginning of injection $\left(I_{o}=0 \mathrm{~A}\right)$, at the end of injection with maximum current at low energy, and the physics/collision phase at higher energy. The beam and RF station dynamics change considerably during these steps.

At the nominal current of $0.58 \mathrm{~A}$ the LHC klystrons will be operating at approximately $297 \mathrm{~kW}$ close to the maximum value of $330 \mathrm{~kW}$. Therefore, the LHC klystrons are operated close to saturation. In order to separate possible saturation effects in this work, studies are conducted at both the nominal current of $0.58 \mathrm{~A} \mathrm{DC}$ and at the more conservative value of $0.3 \mathrm{~A}$ DC.

\section{High-Level RF settings}

For the studies presented in this Chapter, the cavity voltage $V$ and loaded quality factor $Q_{L}$ are set to $1 \mathrm{MV}$ and 20,000 respectively during injection and to $2 \mathrm{MV}$ and 60,000 during the physics phase.

The LHC beam current is irregular around the ring due to the bunch pattern and 
the voltage is kept constant over the turn thanks to the RF and 1-Turn Feedback systems. If the cavity were to be detuned for minimum klystron power with the beam present, then the klystron would have to switch between two power levels in the presence and absence of beam. To minimize klystron power over one turn, the cavity is set using the half-detuning algorithm during LHC operation [68], [69], [70]. The half-detuning algorithm decreases the instantaneous power of the klystron in the absence of beam and keeps the klystron power constant during the changes in the beam pattern. The same scheme is used in the simulations presented in this work.

\section{LLRF parameters}

The LLRF parameters adjusted during this work are the feedback gain, the 1-Turn delay, and the loop phase. The 1-Turn feedback loop gain and phase are not adjusted during operations, but are set to $20 \mathrm{~dB}$ and $0^{\circ}$ respectively.

Table 7.1 shows the cavity detuning $f_{d}$ and the LLRF parameters for each operational scenario considered, as described above. It should be noted that $G$ and $\phi$ are reference values that define relative changes to the parameters set in the hardware. Using the simulation, a $9 \mathrm{~dB}$ gain margin was estimated for the 1-Turn feedback loop, close to the $10 \mathrm{~dB}$ value reported during development [71]. The 1-Turn feedback loop delay $\tau_{d}$ is optimized during operations. For the simulation the optimal value was $87.8 \mu \mathrm{s}$. As expected, the LLRF configuration changes significantly during the LHC

\begin{tabular}{|c|c|c|c|c|c|c|c|}
\hline & Beam & \multicolumn{4}{|c|}{ High Level RF } & \multicolumn{2}{c|}{ LLRF } \\
\hline Conf. & $I_{o}(\mathrm{~A})$ & $V_{o}(\mathrm{MV})$ & $Q_{L}$ & $P_{k l}(\mathrm{~kW})$ & $f_{d}(\mathrm{kHz})$ & $G(\mathrm{~dB})$ & $\phi$ \\
\hline Inj. Beg. & 0 & 1 & $2 \mathrm{e} 4$ & 139 & 0 & 17.44 & $2.4^{\circ}$ \\
\hline Inj. End & 0.3 & 1 & $2 \mathrm{e} 4$ & 149 & -2.7 & 17.44 & $2.4^{\circ}$ \\
\hline Physics & 0.3 & 2 & $6 \mathrm{e} 4$ & 216 & -1.35 & 22.35 & $5^{\circ}$ \\
\hline Inj. End & 0.58 & 1 & $2 \mathrm{e} 4$ & 177 & -5.3 & 17.82 & $2.6^{\circ}$ \\
\hline Physics & 0.58 & 2 & $6 \mathrm{e} 4$ & 298 & -2.65 & 23.3 & $8^{\circ}$ \\
\hline
\end{tabular}

Table 7.1: RF and LLRF parameters for beginning/end of injection and physicscollision configurations considered in this Chapter.

ramp, whereas during injection the LLRF parameters are essentially unchanged (low 
beam loading), and only the klystron forward power shows a noticeable effect.

The LHC optimal configuration tools inject noise to the RF station for a brief period of time to characterize the $\mathrm{RF}$ station through a transfer function measurement around an operation point. Due to beam emittance blowup concerns, the optimal configuration tools will not be used in the presence of beam according to the the current operational plan. As a result, with the current operation plan the LLRF is optimized with no beam before injection, and then the LLRF is kept at the same settings throughout the LHC operation, thus significantly reducing the performance of the RF station compared to a situation where the parameters are adjusted at each stage. To estimate the effect of this operational scenario, the simulation is run using the optimal LLRF parameters calculated at $I_{o}=0$ but at the physics configuration with $I_{o}=0.3 \mathrm{~A}$. The results are reported under "Non-optimal Physics". In a hypothetical scenario, it would be possible to estimate the optimal LLRF parameters for physics/collision using the simulation, and then use those settings during injection, ramping, and physics. This scenario will have reduced performance at injection, and the corresponding results are reported as "Non-optimal Injection End".

\subsubsection{Noise Thresholds for Longitudinal Beam Diffusion}

To determine the noise power threshold at the output of the LLRF and the modulator, the simulation is set to the configurations of interest and the estimated transfer function $H_{g}(f)$ between the noise (i or q) and the phase of the cavity voltage is measured. As described in Equation 7.1, the transfer functions for all sources and channels should be measured for each operational condition. The estimated transfer function between the RF accelerating voltage phase and the noise at the q channel at the input of the modulator is shown in Figure 7.3 as an example. Assuming a wideband noise source of power constant spectral density $\left(N^{m}\right)^{2}$ for source $m$, Equation 7.11 can be simplified to

$$
\sigma_{z}^{2}=\frac{2 c^{2}}{\omega_{R F}^{2}}\left(N^{m}\right)^{2} \int_{0}^{\infty} B^{m}\left(B^{m}\right)^{H} d f
$$




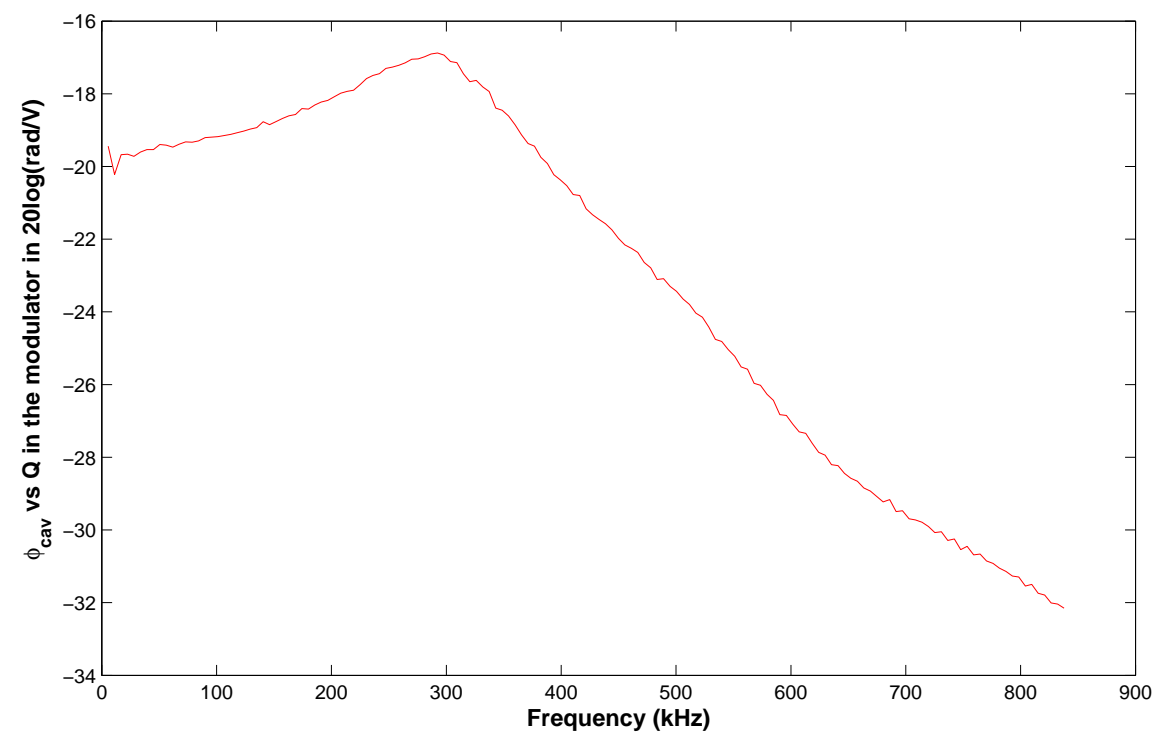

Figure 7.3: (Color) The estimated transfer function between the RF accelerating voltage phase and the noise at the q channel at the input of the modulator $\frac{\phi_{c a v}(f)}{n_{q}^{M o d}(f)}(\mathrm{rad} / \mathrm{V})$.

Then, it is possible to estimate the ratio between the phase modulation in the RF accelerating voltage and the noise source for each of the configurations. This ratio is calculated using the estimated transfer function from the whole band which is aliased over the frequency band from the RF operating frequency out to the first revolution harmonic following Equations 7.9, 7.10, and 7.11.

The power spectral density $N_{o}^{2}$ for one channel in a single RF station that achieves an equilibrium bunch length equal to the initial condition for source $m$, can be calculated using this information, so that it is possible to calculate the noise spectral density for each source that achieves that bunch length

$$
N_{o}^{2}=\frac{\sigma_{z_{o}}^{2} \omega_{R F}^{2}}{2 c^{2} \int_{0}^{\infty} B^{m}\left(B^{m}\right)^{H} d f}
$$

for $\sigma_{z_{o}}$ equal to 11.24 and $7.55 \mathrm{~cm}$ during injection and physics respectively. The results of these calculations for all the configurations of interest are shown in Table 7.2 and 7.3 for injection and physics configurations respectively. As expected, the 


\begin{tabular}{|c|c|c|c|c|}
\hline Configuration & \multicolumn{2}{|c|}{$V_{\text {Modulator }}$} & \multicolumn{2}{|c|}{$V_{\text {LLRF }}$} \\
\hline & $\mathrm{i}$ & $\mathrm{q}$ & $\mathrm{i}$ & $\mathrm{q}$ \\
\hline Inj. Begin & 72.8 & 7.9 & 32.7 & 1.3 \\
\hline Inj. End 0.58A & 82.5 & 8.4 & 9.2 & 1.2 \\
\hline Inj. End 0.3A & 98.2 & 8.0 & 16.6 & 1.3 \\
\hline Non-Optimal Inj. End 0.3A & 33.2 & 6.4 & 2.1 & 0.57 \\
\hline
\end{tabular}

Table 7.2: Modulator and LLRF noise threshold in $\mathrm{pV} / \sqrt{\mathrm{Hz}}$ for injection configurations. These values correspond to the RF settings shown in Table 7.1.

\begin{tabular}{|c|c|c|c|c|}
\hline Configuration & \multicolumn{2}{|c|}{$V_{\text {Modulator }}$} & \multicolumn{2}{|c|}{$V_{\text {LLRF }}$} \\
\hline & $\mathrm{i}$ & $\mathrm{q}$ & $\mathrm{i}$ & $\mathrm{q}$ \\
\hline Physics 0.58A & 1.05 & 0.27 & 0.03 & 0.018 \\
\hline Physics 0.3A & 2.41 & 0.18 & 0.12 & 0.018 \\
\hline Non-optimal Physics 0.3A & 2.11 & 0.21 & 0.36 & 0.029 \\
\hline
\end{tabular}

Table 7.3: Modulator and LLRF noise threshold in $\mu \mathrm{V} / \sqrt{\mathrm{Hz}}$ for physics configurations. These values correspond to the RF settings shown in Table 7.1.

noise threshold is significantly lower for the injection configurations, since the synchrotron radiation damping is more than three orders of magnitude lower. The very low thresholds for the injection configurations are not a reason for concern though, since the beam is kept in this condition for a short time. On the other hand, the large sensitivity on the synchrotron radiation and consequently on the beam energy levels implies that the planned low energy operation at $3.5 \mathrm{TeV}$ will reduce the noise threshold limits. Furthermore, one can see the wide variation with RF configuration and input channel ( $\mathrm{i}$ or $\mathrm{q}$ ), as expected from the synchronous phase of $\approx 180^{\circ}$. Table 7.2 shows the impact of the different configurations: the LLRF noise threshold is scaled by a factor of two when the LLRF is operated with the physics configuration during injection (the noise threshold decreases to $0.57 \mathrm{pV} / \sqrt{\mathrm{Hz}}$ from the optimal $1.3 \mathrm{pV} / \sqrt{\mathrm{Hz}}$ ). Using the non-optimal configurations lowers the noise threshold as anticipated. On the other hand, in Table 7.3 there is a factor of 1.6 increase of the noise threshold when the LLRF is operated with the injection settings during physics (from 0.018 to $0.029 \mathrm{pV} / \sqrt{\mathrm{Hz}})$. This small increase though results in a substantial cost to 
beam stability, since the LLRF gain - and consequently the fundamental impedance reduction - is now reduced by $5 \mathrm{~dB}$.

As explained above, these results correspond to only one active noise source and channel at the time. They also represent only one of the eight stations per beam. Therefore, a scheme has to be devised to determine the final threshold. It is straightforward to show that the total power spectral density at the accelerating voltage phase is given by the sum over all channels and sources. Assuming uncorrelated wideband noise sources of varying constant spectral densities and using Equation 7.1, Equation 7.12 becomes

$$
\begin{aligned}
\sigma_{z}^{2} & =8 \frac{2 c^{2}}{\omega_{R F}^{2}} \sum_{m}\left(N^{m}\right)^{2} \int_{0}^{\infty} B^{m}\left(B^{m}\right)^{H} d f \\
\frac{\sigma_{z}^{2}}{\sigma_{z_{o}}^{2}} & =8 \sum_{m} \frac{\left(N^{m}\right)^{2}}{\left(N_{o}^{m}\right)^{2}}
\end{aligned}
$$

Therefore, the values presented in Tables 7.2 and 7.3 provide the weighting coefficients for the contributions of the individual noise sources to the equilibrium bunch length. As such, the noise contributions are dominated by the source with the lower threshold or with a significantly higher noise power.

Equation 7.13 can be very helpful for the system designer, since the values $N^{m}$ can be set based on the technical challenges related to reducing the noise levels of each source. These noise levels define the design specifications for the LLRF and Modulator boards and can be compared with the expected noise levels of the architecture. Dedicated measurements will be necessary to compare with the noise of the actual implementation and verify the calibration of the simulation signals.

Some initial measurements of the noise spectrum at the output of the LHC LLRF Feedback system are shown in Figures 7.4 (wideband) and 7.5 (narrowband) ${ }^{1}$. These measurements were conducted with the LLRF feedback board input terminated to 50 $\Omega$. These noise levels should be comparable to the levels at the input of the Modulator.

\footnotetext{
${ }^{1}$ The source of the spectral lines in these figures has not been identified. Even though they show up consistently in this pickup, they do not correlate with signals in the cavity probe. More measurements in the absence of beam or with the RF input terminated could help determine the source of these spectral lines or show that they are an artifact of the processing
} 


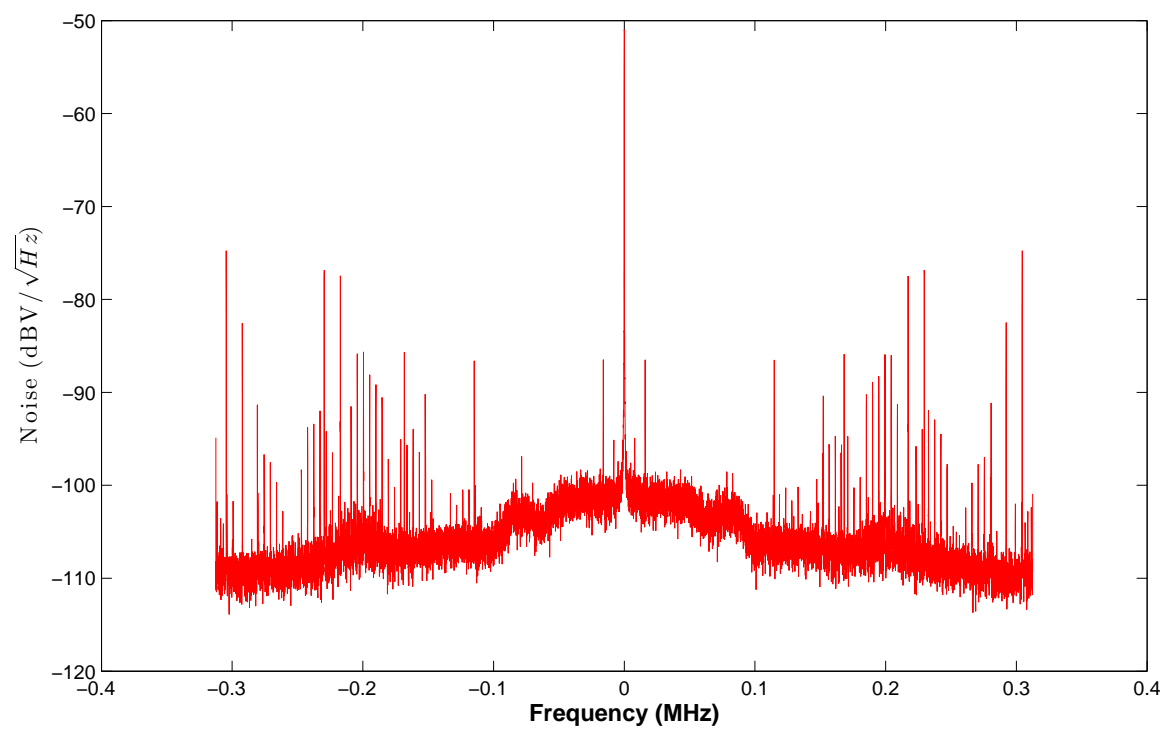

Figure 7.4: Noise power spectral density at the output of the LHC RF Feedback for channel Q(wideband).

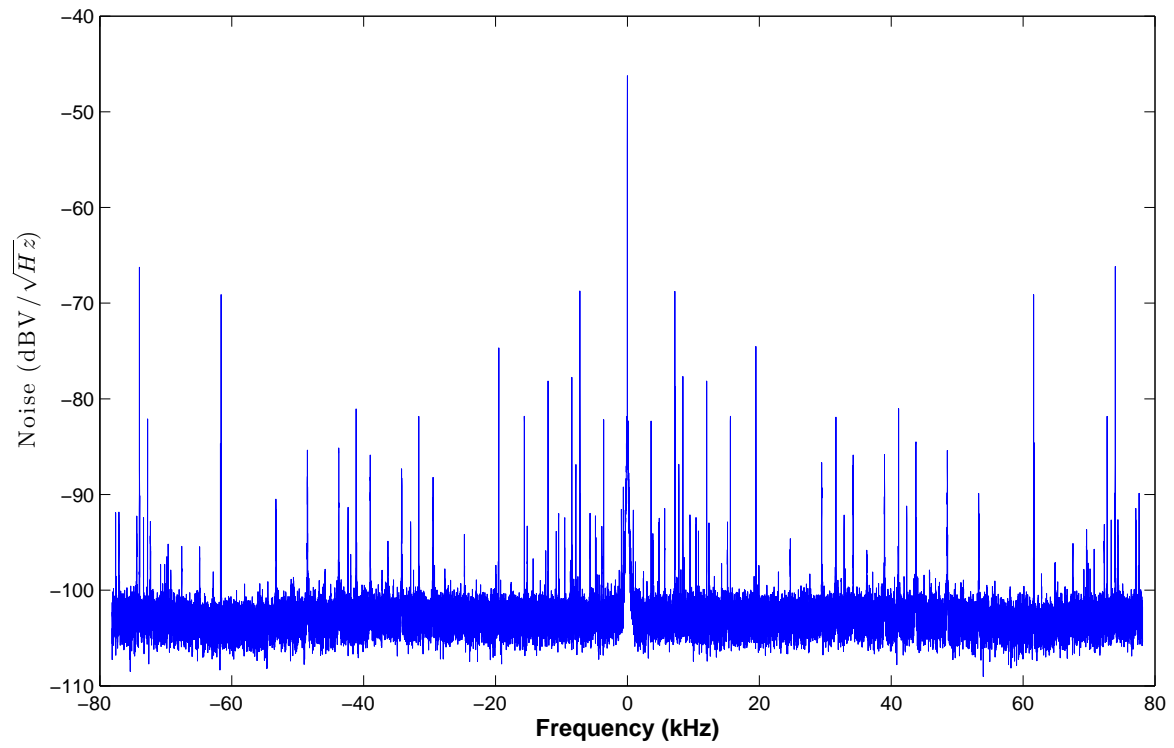

Figure 7.5: Noise power spectral density at the output of the LHC RF Feedback for channel Q(narrowband). 
The value of approximately $7 \mu \mathrm{V} / \sqrt{\mathrm{Hz}}$ in the bandwidth of the accelerating cavity is higher than the thresholds in Table 7.3, so a slow growth of longitudinal emittance is anticipated.

\subsection{Multi-bunch Stability Studies}

Various studies have been conducted to evaluate the longitudinal coupled-bunch instabilities at the LHC [72], [73]. These studies do not include the cavity fundamental impedance nor consider the effect of the LLRF impedance reduction feedback system though. Using the time-domain simulation and related models presented in Section 7.4, it is possible to estimate the effective impedance presented to the beam by the RF station for any configuration. The coupled-bunch instabilities can then be computed to study the bunch centroid stability, position, and motion due to multibunch coupling as a function of the RF configurations.

An advantage of the time-domain simulation approach is the ability to vary individual LLRF feedback parameters and determine their effect on the beam stability. As a result, the sensitivity on individual RF parameters can be estimated, and the possible tradeoffs between beam and RF station stability can be investigated. The related results are presented in Subsection 7.5.2.

Impedance reduction is of fundamental importance at the LHC since there is no dedicated bunch-by-bunch longitudinal feedback system. The substantial bunch length leads to stability through Landau damping. The effective cavity impedance though depends strongly on the LLRF configurations. In this section, the coupledbunch instabilities are investigated as a function of the LLRF configurations to determine the stability margins for the LHC.

The effective cavity impedance is computed using a linearized model of the RF station and LLRF feedback around the operation point [47], based on the system operating points determined from the nonlinear simulation tools. For operation with $V_{o}=2 \mathrm{MV}$ and $Q=60 \mathrm{k}$, the analog/digital loop and the 1-Turn feedback provide a reduction of the superconducting cavity impedance of about $50 \mathrm{~dB}$ around mode 0 , as expected. 


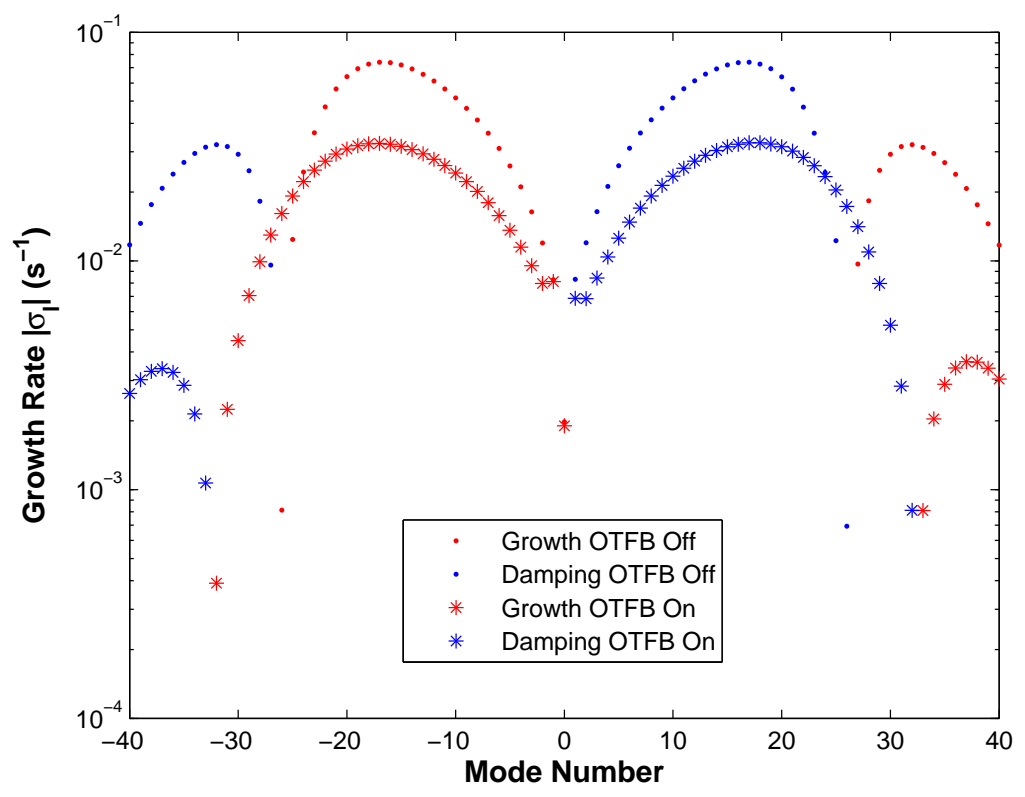

Figure 7.6: Modal Growth Rates for configuration Injection End 0.3 A with 1-Turn Feedback (OTFB) on or off.

Using the estimated impedance and assuming a gaussian bunch, the growth rate $\sigma_{l}$ and tune shift $\Delta \omega_{l}$ can be computed for each coupled-bunch mode $l[74]$

$$
\sigma_{l}+j \Delta \omega_{l}=\frac{\eta q I_{o}}{2 \beta^{2} \omega_{s} E_{o} T_{o}} \sum_{p=-\infty}^{\infty} Z(\omega) \omega e^{\sigma_{\tau}^{2} \omega^{2}}
$$

where $\eta$ is the slip factor, $q$ is the charge of a proton, $I_{o}$ is the DC beam current, $\beta$ is the ratio of the particle speed to the speed of light, $\omega_{s}$ is the synchrotron frequency, $E_{o}$ is the beam energy, $T_{o}$ is the revolution period, $Z$ is the estimated RF station impedance contributed from all 8 stations per beam, and $\sigma_{\tau}$ the bunch length in time units. The impedance is evaluated at frequencies $\omega=(p h+l) \omega_{0}+\omega_{s}$ with $\omega_{o}$ the angular revolution frequency, $h$ the harmonic number, $l$ the mode number, and p any integer. Figures 7.6 and 7.7 show an example of the resulting modal growth rates and tune shifts for configuration Injection End 0.3 A with the 1-Turn Feedback on or off. The reduction of the growth rates and tune shifts for all lower order modes - 


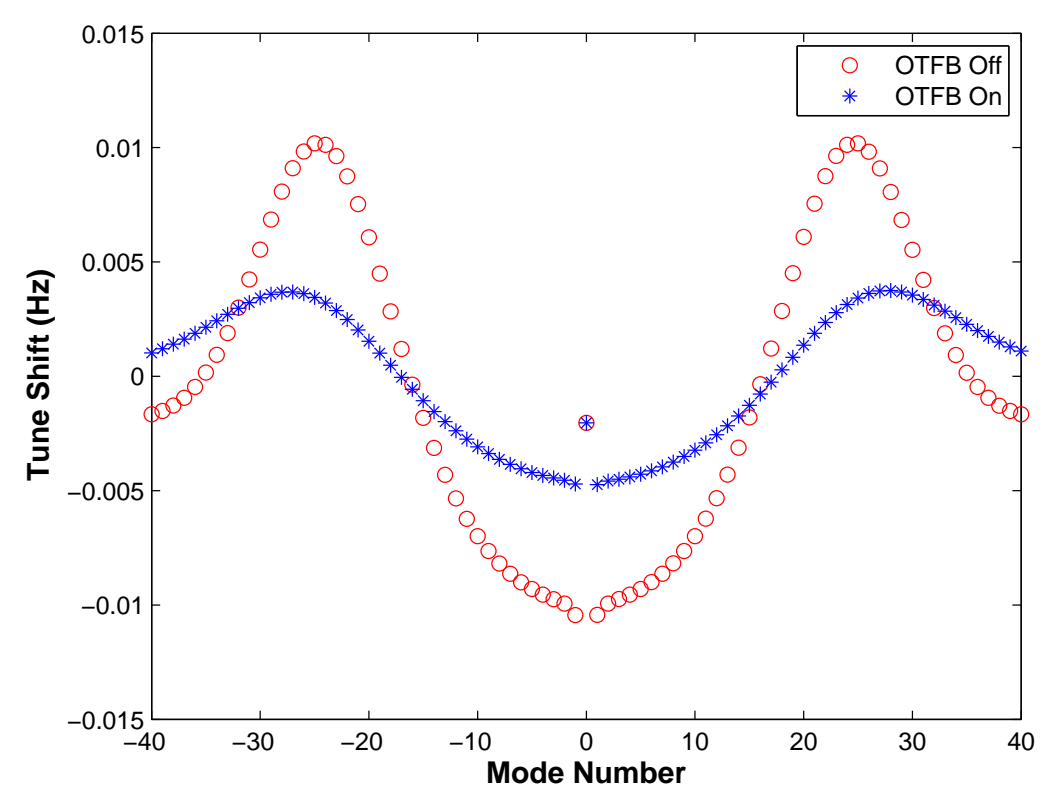

Figure 7.7: Tune shift for configuration Injection End 0.3 A with 1-Turn Feedback (OTFB) on or off.

except for mode 0 - is evident.

\subsubsection{Stability Criterion}

The interaction between the cavity fundamental impedance and the beam produces growth rates in the order of seconds when the LLRF feedback system is operating. Even though these growth rates are very slow - tens of thousands of turns - they are critical, because the synchrotron damping time is in the order of hours (approximately 50,000 and 13 hours for injection and physics respectively). Since there is no bunchby-bunch feedback system, stability is determined by Landau damping - a physical process which stabilizes the otherwise unstable ensemble of oscillating particles due to a spread of their natural frequencies caused by the non-linearity of the RF voltage. To determine stability, the criterion defined in [75], [76], [77] is used with the same safety margins:

$$
\sigma_{l}<\frac{\Delta \omega_{s}}{4}
$$


where $\Delta \omega_{s}$ is the synchrotron frequency spread within the bunch. The synchrotron frequency spread is given by

$$
\Delta \omega_{s}=\omega_{s} \frac{\pi^{2}}{16}\left(\frac{h L}{2 \pi R}\right)^{2}
$$

where $h=35640$ is the harmonic number, $L$ is the total bunch length $\left(4 \sigma_{z}\right)$, and $R=4242.893 \mathrm{~m}$ is the LHC radius. Since the LHC rms bunch length is 11.24 and $7.55 \mathrm{~cm}$ during injection and collision respectively, $\Delta \omega_{s} / 4$ is equal to 1.19 and 1.55 for these two cases.

The growth rate of the most unstable mode $\sigma_{\max }$ and the maximum tune shift $\Delta f_{\max }$ for each configuration are reported in Table 7.4. Comparing the results from

\begin{tabular}{|c|c|c|}
\hline Configuration & $\sigma_{\max }\left(\mathrm{s}^{-1}\right)$ & $\Delta f_{\max }(\mathrm{Hz})$ \\
\hline Inj. End 0.58 A & 0.055 & 0.0071 \\
\hline Physics 0.58 A & 0.0041 & 0.0011 \\
\hline Inj. End 0.3 A & 0.033 & 0.0047 \\
\hline Physics 0.3 A & 0.0061 & 0.0009 \\
\hline Non-optimal Inj. End 0.58 A & 0.083 & 0.0099 \\
\hline Non-optimal Physics 0.3 A & 0.019 & 0.0044 \\
\hline
\end{tabular}

Table 7.4: Growth Rates of the most unstable mode and maximum tune shifts for each configuration, with the 1-Turn Feedback on.

Table 7.4 with the threshold margins of 1.19 and 1.55 for injection and physics, the fastest growth rate is at least a factor of twenty smaller than the stability criterion threshold for all configurations. It is also obvious that the configurations at physics have lower growth rates than those at the end of injection due to the almost twentyfold increase in beam energy. It should be mentioned that the estimated impedance driven tune shifts are insignificant compared to the frequency spread due to the nonlinearity of the RF voltage.

It is important to note the significant effect that changes of the LLRF configuration can have on the modal growth rates, as can be seen for the almost threefold increase in growth rates with the non-optimal configurations. Even though the stability threshold is not crossed, it is important to notice the importance of the optimal LLRF tuning 
not only for the RF station stability, but also for beam stability. The tuning can be even more critical for lower beam energies. As seen from Equation 7.14, the growth rates are inversely proportional to the beam energy $E_{o}$. During the initial LHC runs, the beam energy has been and will be kept at much lower levels than the nominal 7 $\mathrm{TeV}$. To maintain the margin level calculated above, the current $I_{o}$ should be scaled similarly. For example, an LHC configuration with the nominal current of $0.58 \mathrm{~A}$ at an energy of $1 \mathrm{TeV}$ will cause coupled-bunch instabilities with growth rates seven times higher than those presented in Table 7.4, and would probably lead to beam loss. Once again, operation at lower energies can have negative effects on the longitudinal beam dynamics.

\subsubsection{Growth Rate Sensitivity to LLRF Parameters}

One of the important features of the LHC time-domain simulation is the ability to study alternative configurations of the RF and LLRF system, without requiring time from the real machine. As such, it can be used to analyze the sensitivity of the modal growth rates to variations of the LLRF parameters. These studies provide insight on the limits of the implementation, on the operational margins, and on the parameters most essential to reliable operations.

Using the configuration at the end of injection with a beam current of $0.3 \mathrm{~A}$ as a reference, each of the following parameters were modified separately to understand their impact in the interaction between the RF station and the beam dynamics: Cavity detuning $f_{d}$, Analog/Digital loop gain $G$, Controller phase $\phi$, and 1-Turn feedback loop gain $G_{c}$ and phase $\phi_{c}$. The variations on each case were determined to correspond to reasonable variations over a run. The system's impedance and corresponding growth rates were estimated for each case. The growth rates of the fastest growing mode for each case are reported in Table 7.5. It is interesting to see the considerable beam stability dependence on the Controller phase and the 1Turn feedback phase. A six-fold increase of the growth rates with a Controller phase rotation of $10^{\circ}$ reduces the margin of operation to a factor of three, which then limits the maximum reliable current for energy levels lower than $7 \mathrm{TeV}$. This analysis shows 


\begin{tabular}{|c|c|c|c|}
\hline Parameter & Adjustment & Growth Rate & Change \\
\hline Nominal Value & - & 0.033 & - \\
\hline$f_{d}$ & $\pm 1 \mathrm{kHz}$ & $0.038 / 0.028$ & $+15 /-15 \%$ \\
\hline$G$ & $\pm 3 \mathrm{~dB}$ & $0.028 / 0.043$ & $-16 /+31 \%$ \\
\hline $\boldsymbol{\phi}$ & $\pm 10^{\circ}$ & $0.23 / 0.19$ & $+\mathbf{5 9 0} /+\mathbf{4 9 0} \%$ \\
\hline$G_{c}$ & $\pm 3 \mathrm{~dB}$ & $0.026 / 0.039$ & $-20 /+20 \%$ \\
\hline$\phi_{c}$ & $\pm 10^{\circ}$ & $0.12 / 0.10$ & $+270 /+220 \%$ \\
\hline
\end{tabular}

Table 7.5: Growth Rate Sensitivity on LLRF parameters.

the critical importance of careful tuning of the LLRF in cases where the beam stability margin is limited.

It is not surprising that there are changes in LLRF parameters that improve beam stability. The LLRF is tuned in a manner that maximizes the stability of both the beam and the RF-LLRF loop. For example, the cavity detuning $f_{d}$ is set to minimize the average klystron power. The Analog/Digital loop gain as well as the 1-Turn feedback loop gain are set to achieve predetermined gain margins. Therefore, a trade-off exists between beam and loop stability.

A similar study was performed for variations of the 1-Turn feedback delay. The total delay in the 1-Turn feedback loop is set by a coarse delay of $100 \mathrm{~ns}$ increments, and a fine delay of 10 ps increments. In our study, no considerable effects on the estimated growth rates were experienced even when the delay was changed by a few hundred ns (corresponding to multiple taps of the coarse setting). On the other hand, a shift of even a few tens of nanoseconds is sufficient to bring RF station instability. Thus, optimal tuning of the 1-Turn feedback delay might not be critical for beam stability directly, but it is essential for RF station stability, and consequently for reliable operation with beam. 


\section{Chapter 8}

\section{RF Noise Effects on LHC Beam Diffusion}

The theoretical formalism presented in Section 7.3, suggests that the noise experienced by the beam depends on the cavity phase noise power spectrum, aliased due to the periodic sampling of the accelerating voltage $V$ by the beam. Additionally, the dependence of the RF accelerating cavity noise spectrum on the LLRF configurations has been predicted using the LHC time-domain simulations and models described in Chapter 6. In this Chapter, measurements at the LHC supporting the above theoretical formalism and simulation predictions are presented [78], [79].

Section 8.1 briefly describes the RF system with an emphasis on beam diffusion. Section 8.2 presents the noise generating RF components, and identifies the element that dominates the longitudinal beam emittance blowup effects. Quantitative experiments of the beam diffusion dependence on RF noise are shown in Section 8.3. Section 8.4 includes an element-wise study of the RF Feedback noise contributions. Finally, Section 8.5 studies the LLRF noise dependence on the controller gain settings and compares the LLRF noise spectrum estimated by the time-domain simulations with data from LHC. 


\subsection{System Description}

A simplified block diagram of the LHC RF system is shown in Figure 8.1. The elements in green have been described in Section 6.2.1. The LLRF feedback system

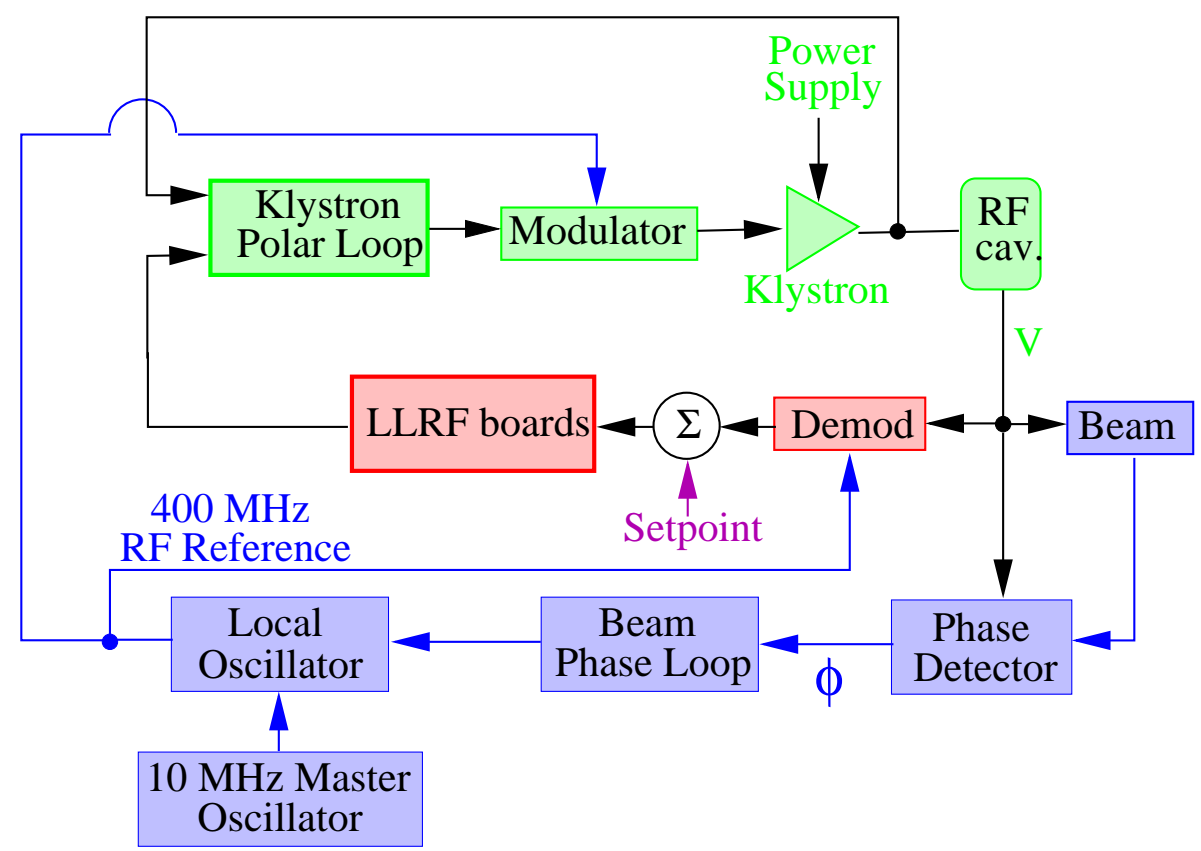

Figure 8.1: Simplified block diagram of the RF system with Beam Phase Loop.

processing takes place in baseband. A $400.8 \mathrm{MHz} \mathrm{RF}$ reference signal is created by the Local Oscillator to modulate/demodulate the baseband signals to/from RF frequencies. The RF reference is created from a $10 \mathrm{MHz}$ Master Oscillator, which synchronizes the LHC with the Super Proton Synchrotron (SPS) and Proton Synchrotron (PS). The Beam Phase Loop (BPL) is a narrow bandwidth loop that acts on the Local Oscillator to achieve damping of mode zero beam motion around the synchrotron frequency. As such, it damps out longitudinal motion around the synchronous phase, motion driven by noise in the RF system or other mechanisms. The bandwidth of the BPL is limited by the revolution frequency $f_{r e v}$ of $11.245 \mathrm{kHz}$.

The input to the BPL is the beam phase error signal $\phi$, a measure of the average deviation of the phase difference between the beam and the cavity sum over a turn. The BPL processes $\phi$ to control the phase of the Local Oscillator so that $\phi$ is reduced 
around the synchrotron frequency $f_{s}$. The BPL includes an adjustable gain and does not act around DC. The substantial reduction with the BPL on is shown in Figure 8.2,

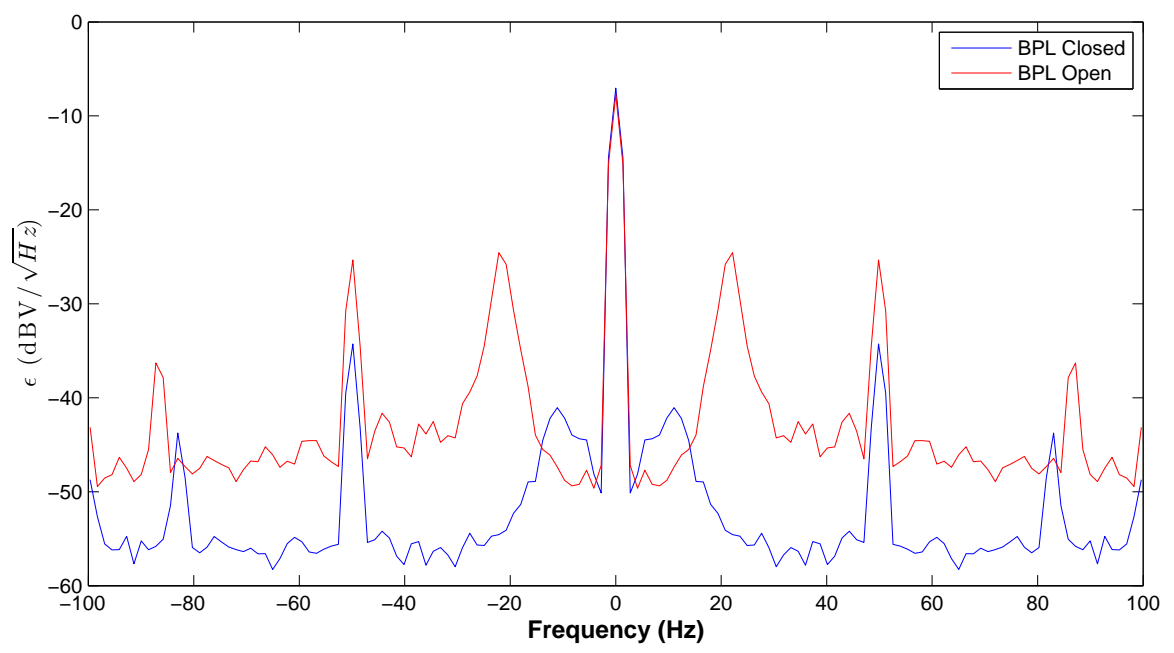

Figure 8.2: Beam 1 phase error with BPL on/off.

which shows the spectrum of $\phi$ with the BPL open or closed. With the BPL open there is a substantial phase difference at the synchrotron frequency of about $23 \mathrm{~Hz}$ due to the synchrotron oscillations.

\subsection{RF Noise Dominating Component}

Before studying the effect of the accelerating cavity phase noise on the longitudinal beam emittance, it is important to determine the RF components that impact the accelerating cavity noise spectrum. Initial measurements of the power spectral density of the accelerating cavity phase noise during operations with $3.5 \mathrm{TeV}$ beam showed that the noise noise is dominated by the $\mathrm{RF}$ reference up to about $300 \mathrm{~Hz}$, and the LLRF controller at higher frequencies. The LLRF controller has an almost flat frequency spectrum up to the bandwidth of the cavity $(\approx 300 \mathrm{kHz})$. The noise from the LLRF electronics increases as $1 / \mathrm{f}$ for frequencies lower than about $50 \mathrm{kHz}$, but the reference noise dominates for frequencies less than $f_{\text {rev }}$ and this effect is not seen. 
Measurements were first conducted with the BPL off. Figure 8.3 shows the phase noise of the Beam 2 cavity sum signal and the $400.8 \mathrm{MHz}$ reference (not regulated by

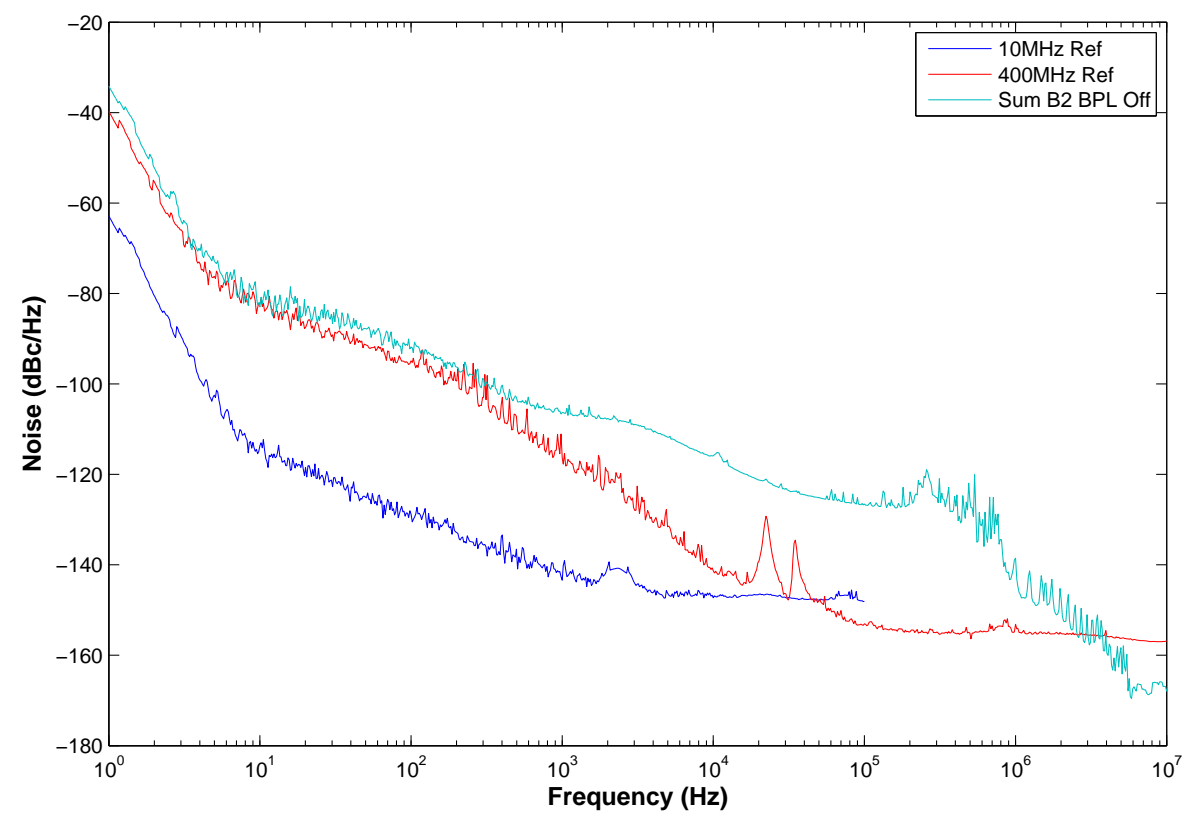

Figure 8.3: Beam 2 cavity sum and RF reference-BPL OFF

the BPL. Available at the LLRF crate), and the $10 \mathrm{MHz}$ reference. It is obvious from this figure that the cavity sum noise follows the $400.8 \mathrm{MHz}$ reference up to approximately $300 \mathrm{~Hz}$. At higher frequencies, the noise is dominated by the LLRF controller. This separation is of course applicable only to this specific technical implementation. Even though the $10 \mathrm{MHz}$ noise levels are very low, they are scaled by a factor of 40 during the up-conversion in the Direct Digital Synthesizer (DDS), corresponding to the $32 \mathrm{~dB}$ seen in the figure.

Since the BPL acts on the RF reference around the synchrotron frequency, an improvement of the noise spectrum around $f_{s}$ is anticipated with the BPL closed. Figure 8.4 shows this phase noise reduction around the synchrotron frequency of about $23 \mathrm{~Hz}$ due to the BPL. It is interesting to note the increase of the noise levels outside a narrow band around $f_{s}$ and up to the $11.245 \mathrm{kHz}$ bandwidth of the BPL. This increase depends on the BPL gain as will be shown in the next Section. It is 


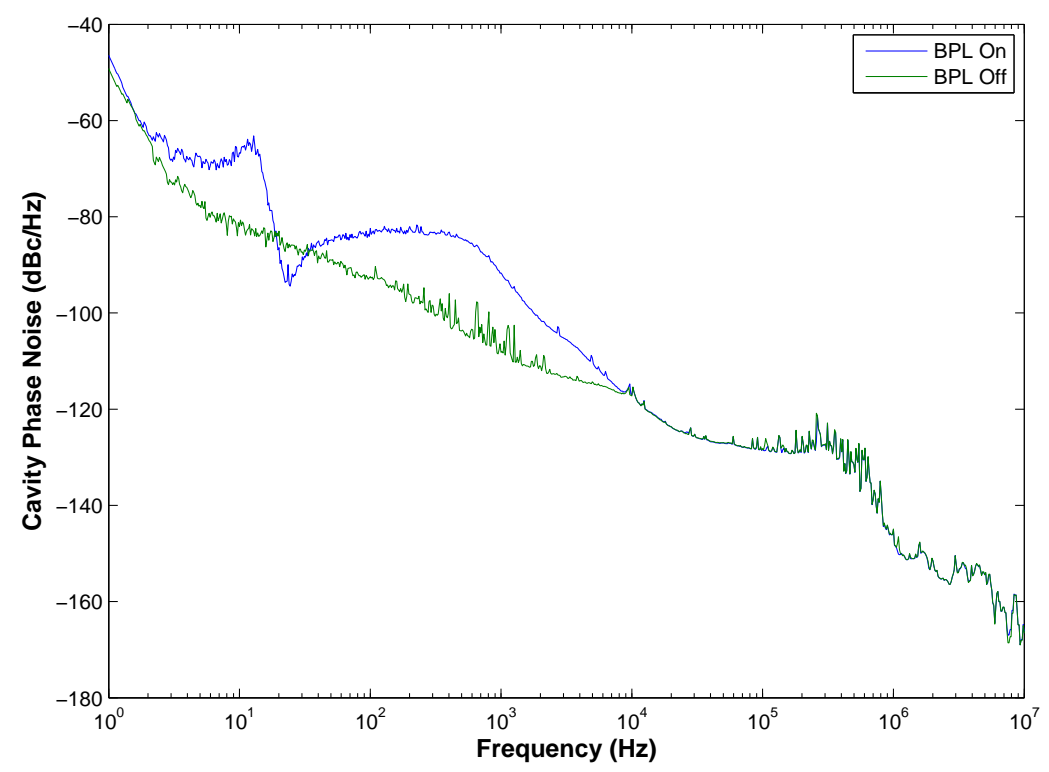

Figure 8.4: Beam 2 cavity sum with BPL On/Off

also important to see that the cavity sum signals are identical outside this bandwidth independently of the state of the BPL, as expected since the LLRF dominates at these frequencies and its configuration has not changed.

\subsection{Beam Diffusion Dependence on RF Noise}

Bunches of initial length of about $11 \mathrm{~cm}$ are injected to the LHC and reduced to about $7.5 \mathrm{~cm}$ during the energy ramp. During the long store, the bunch diffuses longitudinally due to intrabeam scattering and RF noise. The Fokker-Planck formalism is used often to describe the latter effect [66], [80]. This formalism though cannot be applied to colored noise sources and does not include the aliasing effect due to the beam periodicity.

Equation 7.13 was developed in Chapter 7 to estimate the equilibrium bunch 
length $\sigma_{z}$. For simplicity, Equation 7.13 can be rewritten as

$$
\sigma_{z}^{2}=2 \frac{c^{2}}{\omega_{R F}^{2}} \int_{0}^{\infty}\left(H_{\text {beam }}(f) \sum_{k=-\infty}^{\infty} H_{g}\left(f-k f_{\text {rev }}\right)\right)^{2} S_{N}(f) d f
$$

where $S_{N}(f)$ is the the power spectral density of the RF noise sources, $H_{\text {beam }}(f)$ is the beam response, $H_{g}(f)$ the loop response, $\omega_{R F}$ the RF angular frequency, and $c$ the speed of light. The summation represents the aliasing of the spectrum due to the periodicity of the beam. The term $\left(\sum_{k=-\infty}^{\infty} H_{g}\left(f-k f_{\text {rev }}\right)\right)^{2} S_{N}(f)$ represents the accelerating cavity phase noise. This equation applies to one source of noise in the RF system. By summing over all the possible sources, or referring all the noise to one point in the system it is possible to compute the equilibrium bunch length for any RF configuration as was shown in Section 7.4.3.

Since the beam is a very high $\mathrm{Q}$ resonator at the synchrotron frequency $f_{s}-$ as shown in Figure 8.5 - the beam sampled power $P_{n}$ is dominated by the noise

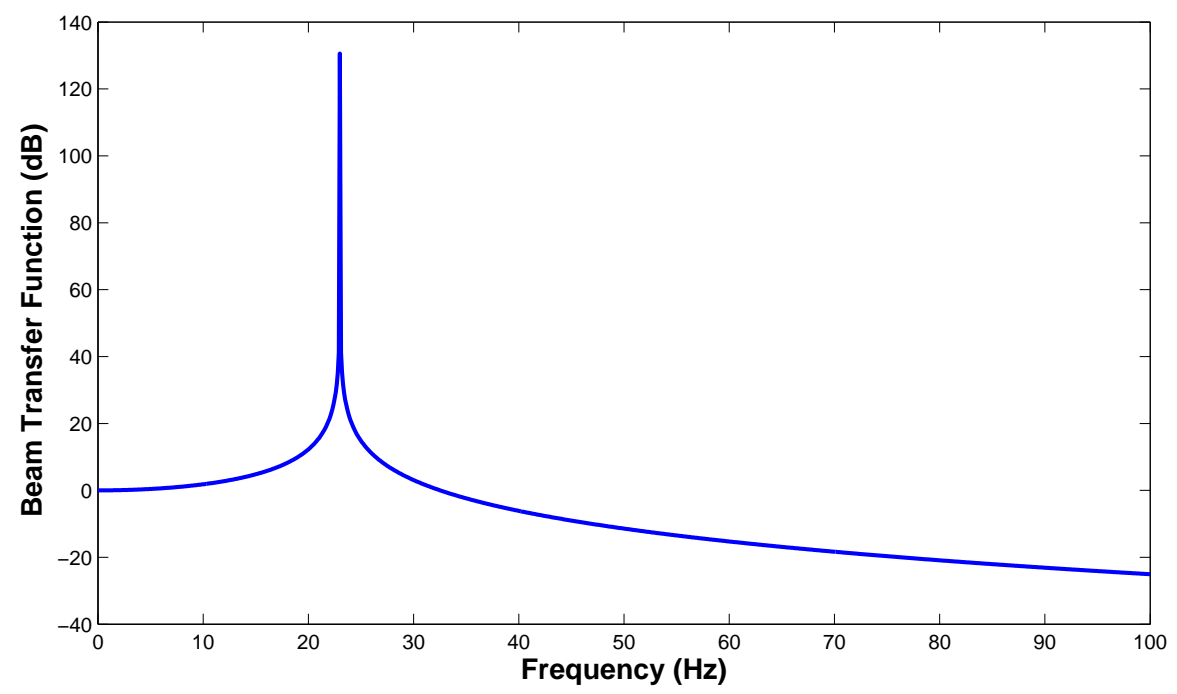

Figure 8.5: LHC beam response.

power spectral density around $f_{s}+k \cdot f_{\text {rev }}$ where $f_{\text {rev }}$ is the revolution frequency and $k$ an integer. The phase power spectral density of the LHC cavity 2 for Beam 1 (cavity 2B1) is shown as an example in Figure 8.6. The beam sampling frequencies 


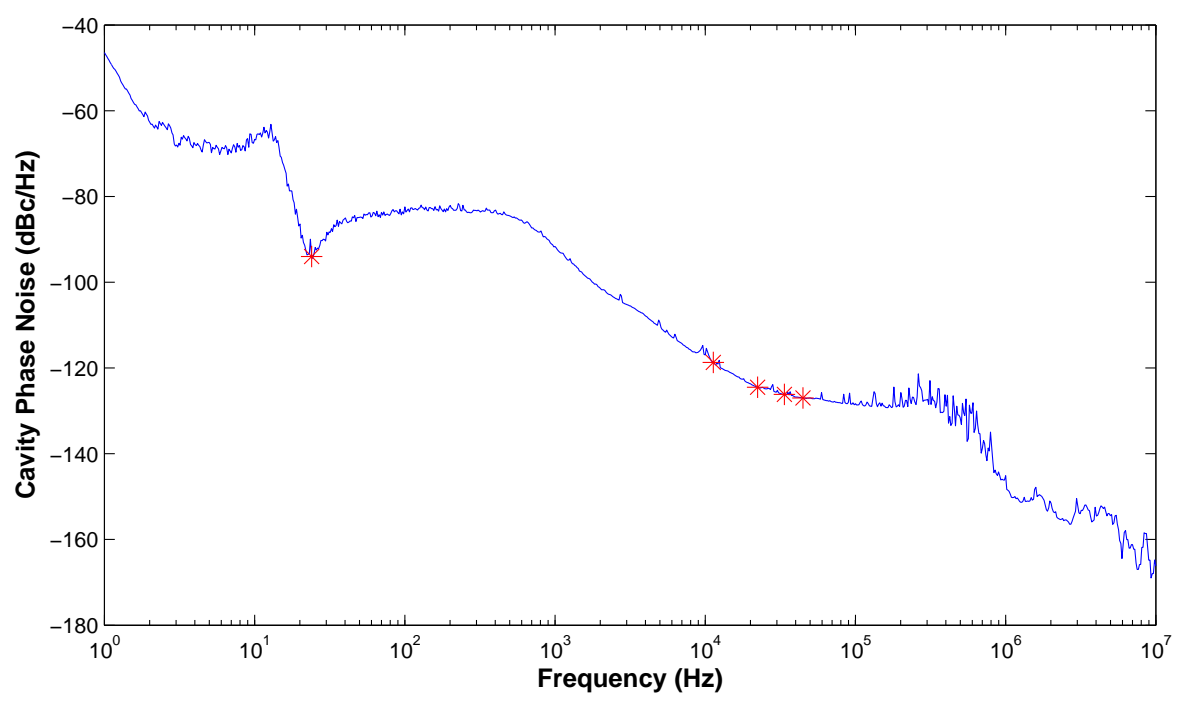

Figure 8.6: Cavity phase noise for cavity 2B1 with $1.5 \mathrm{MV}$, Q of $60 \mathrm{k}, 3.5 \mathrm{TeV}$ beam.

for $k \in\left[\begin{array}{ll}0 & 4\end{array}\right]$ are marked for reference. The total power $P_{n}$ is in fact dominated by the low frequency noise around $f_{s}$ introduced by the RF reference. During all the measurements presented in this work, at least $98 \%$ of the noise power was attributed to the single contribution at $f_{s}$. Therefore, the $\mathrm{RF}$ reference is the single dominating noise contribution to LHC beam diffusion for this particular technical implementation.

Dedicated measurements were conducted to better quantify the relationship between the sampled noise power and the bunch length, and also to better understand the effect of the BPL. During these measurement, the LHC was operating at $3.5 \mathrm{TeV}$, with a non-colliding, single bunch of $910^{9}$ intensity per ring. The BPL gain was varied, which had a significant effect on the noise power spectral density around $f_{s}$, and consequently the noise power sampled by the beam. The wideband spectral density for cavity 6 of Beam 2 (cavity 6B2) is shown in Figure 8.7, as a function of the BPL gain. Figure 8.8 shows the same measurement enlarged around $f_{s}$. Increasing the BPL gain clearly decreases the noise at $f_{s}$.

Figures 8.9 and 8.10 show the effect of the BPL gain settings on the longitudinal bunch length for Beam 1 and 2 respectively ${ }^{1}$. The growth rate of the longitudi- 


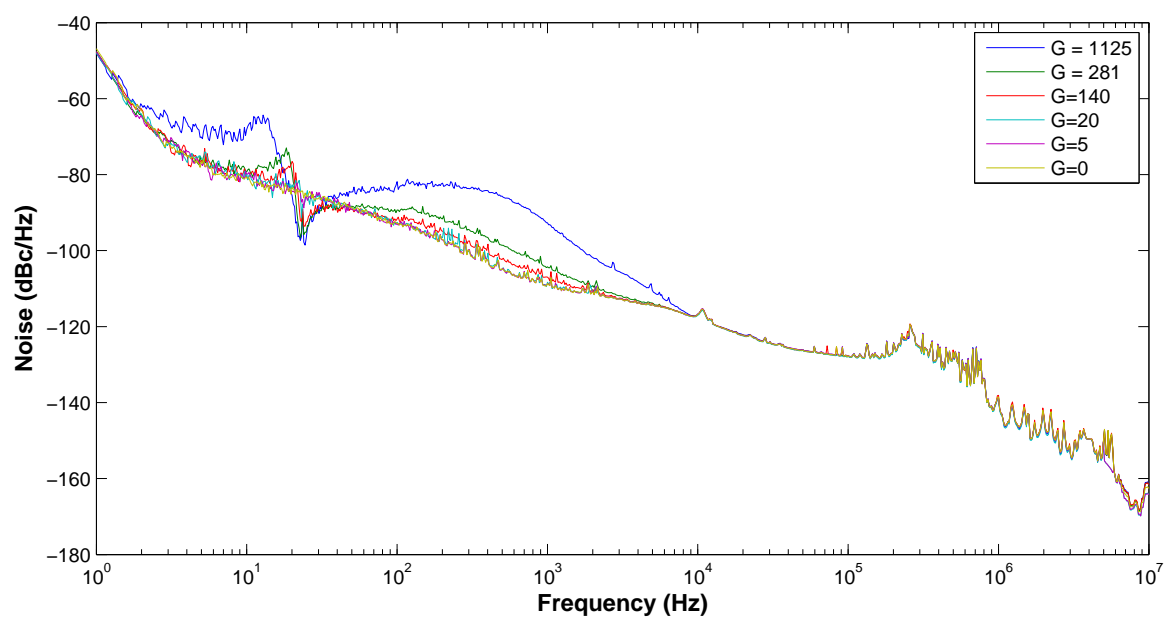

Figure 8.7: Cavity 6B2 noise spectral density with BPL gain.

nal bunch length can be approximated from these figures. Using Equation 8.1, the measured accelerating voltage noise spectrum, and the known beam response, it is then possible to compute the estimated equilibrium bunch length for each setting and compare with the bunch length growth rates. The results are presented in Table 8.1 for Beam 1 and Table 8.2 for Beam 2. One can see the clear correlation between

\begin{tabular}{|c|c|c|c|}
\hline BPL gain & $\sigma_{z}(\mathrm{~cm})$ & $d \sigma_{z} / d t(\mathrm{~cm} / \mathrm{hr})$ & $\begin{array}{c}\text { rms RF Station } \\
\text { Noise }(\mathbf{m r a d})\end{array}$ \\
\hline 1125 & 6.4 & 0.14 & 3.3 \\
\hline 562.5 & 3.5 & 0.17 & 2.2 \\
\hline 70 & 4 & 0.19 & 1.9 \\
\hline 10 & 9.1 & 0.53 & 2 \\
\hline 2 & 12.1 & 2.36 & 1.8 \\
\hline 0 & 16 & 3.20 & 1.9 \\
\hline
\end{tabular}

Table 8.1: Bunch Growth Rate Dependence on BPL gain and Noise Power for B1

the scaled bunch length as estimated by Equation 8.1 and the longitudinal emittance growth, as expected by the developed theoretical formalism ${ }^{2}$. The rms RF station

\footnotetext{
${ }^{1}$ Bunch length data used in this work were provided by the Beam Quality Monitor (BQM) [81]. The BQM reports the $4 \sigma$ bunch length assuming a gaussian bunch.

${ }^{2}$ The first estimated bunch length entry shown in italics in Table 8.1 seems unreasonably high. The reason for this discrepancy is the limited instrument resolution, as seen in Figure 8.8.
} 


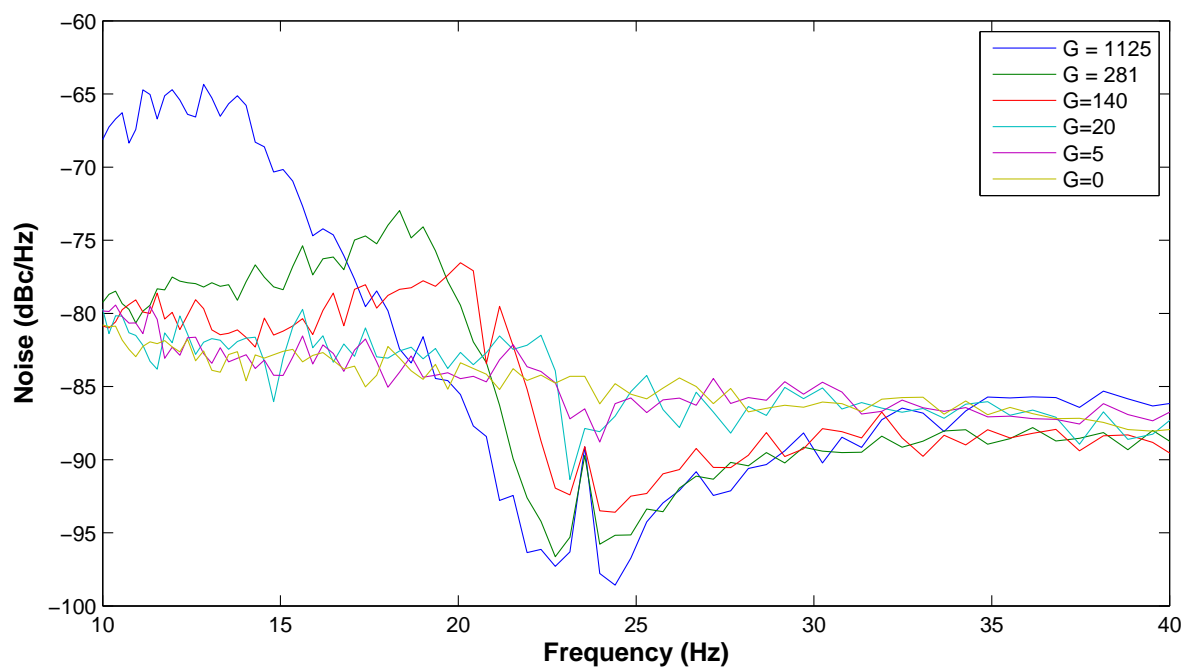

Figure 8.8: Cavity 6B2 noise spectral density with BPL gain.

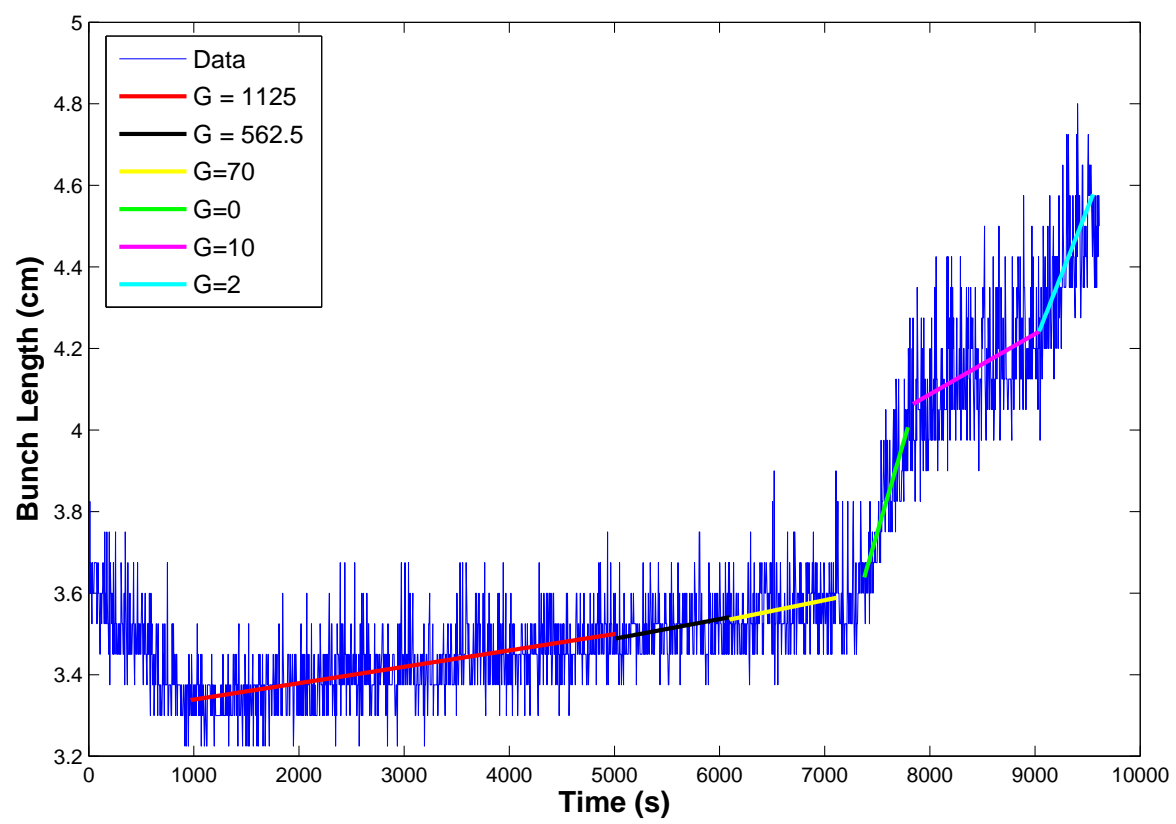

Figure 8.9: B1 Bunch Length with time. 


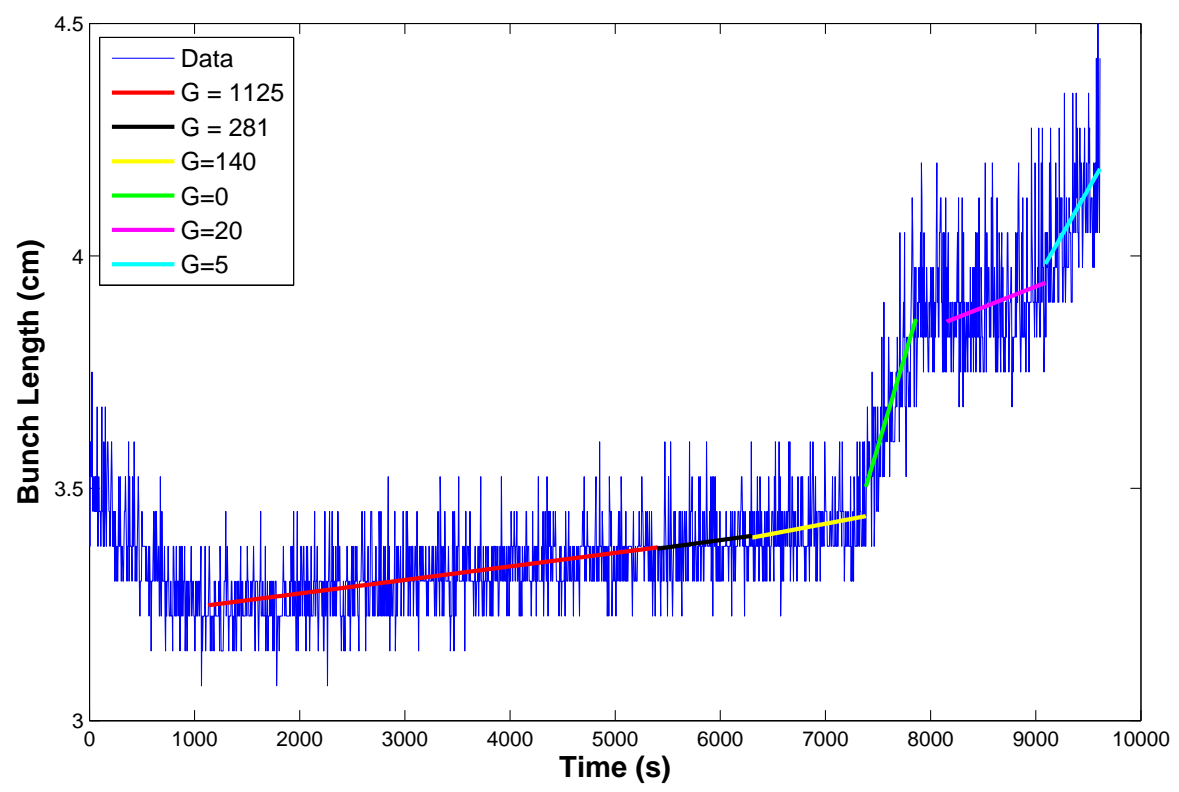

Figure 8.10: B2 Bunch Length with time.

phase noise is computed by integrating the phase noise spectrum as shown in Figure 8.7. This value is also reported to show that it is not a valuable metric for beam dynamics performance, contrary to common practice.

For a BPL gain of more than approximately 30 , there is no significant improvement in beam diffusion. More careful studies will be necessary to exactly quantify this level. There are two reasons for this effect: first, the beam phase loop gets saturated, and second, the longitudinal emittance growth due to intrabeam scattering of about 0.08

\begin{tabular}{|c|c|c|c|}
\hline BPL gain & $\sigma_{z}(\mathrm{~cm})$ & $d \sigma_{z} / d t(\mathrm{~cm} / \mathrm{hr})$ & $\begin{array}{c}\text { rms RF Station } \\
\text { Noise }(\mathbf{m r a d})\end{array}$ \\
\hline 1125 & 4.6 & 0.10 & 3.1 \\
\hline 281 & 5.1 & 0.11 & 2.2 \\
\hline 140 & 7.2 & 0.15 & 2.1 \\
\hline 20 & 8.1 & 0.32 & 2 \\
\hline 5 & 13 & 1.42 & 2.1 \\
\hline 0 & 18.2 & 2.74 & 2.2 \\
\hline
\end{tabular}

Table 8.2: Bunch Growth Rate Dependence on BPL gain and Noise Power for B2 
$\mathrm{cm} / \mathrm{hr}$ becomes comparable to the RF noise induced growth [82], [83] for high gain settings. Finally, the bunch length growth for Beam 1 seems to be higher than that of Beam 2 for the same levels. This discrepancy is probably due to the limited accuracy of the noise estimation as shown in Figure 8.8 or of the bunch length growth estimation shown in Figures 8.9 and 8.10.

\subsection{LLRF Feedback Noise}

It is obvious from Section 8.3 that the RF reference noise dominates the longitudinal emittance growth. Studies are being conducted to identify alternative technical local oscillator implementations to reduce this effect. If a reduced noise RF reference distribution is successfully developed, the noise from the LLRF feedback system would be very important for these considerations, especially since the beam samples the accelerating cavity phase noise almost thirty times in the closed loop bandwidth of the cavity $\left(f_{\text {rev }}=11.245 \mathrm{kHz}\right.$, closed loop bandwidth approximately $300 \mathrm{kHz}$ ). Therefore, a better understanding of the LLRF Feedback noise contributions and its dependence on the controller gain settings could become essential. Studies relating to the LLRF contributions are presented in this section, whereas results showing the effect of the controller settings are reported in Section 8.5.

The LLRF feedback modules are simplified in the block diagram shown in Figure 8.11. There are two main modules, the Analog and Digital module. Each module includes a demodulator to transform the RF cavity signal to baseband and an attenuator to adjust the controller gain. In the digital module, the processing includes an Analog to Digital Converter (ADC) and an FPGA. Because of the limited range of the demodulators, the high RF cavity signal is attenuated significantly before the demodulation. To recover the loop gain, a large gain stage follows the demodulation.

There are multiple electronic components with very diverse noise specifications. For this analysis and due to the topology of the switches, all the LLRF noise sources are referred to four groups: the Analog feedback path with rms noise $y^{2}$, the Digital feedback path up to the switch with rms noise $x^{2}$, the Digital to Analog Converter 


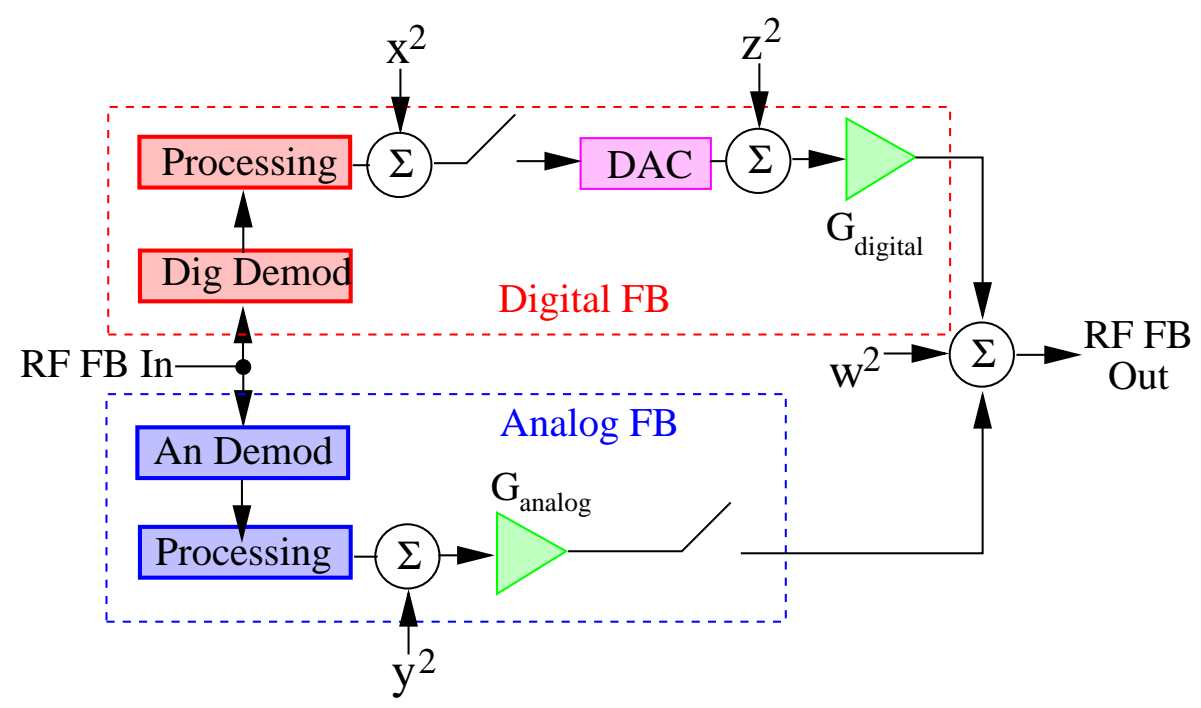

Figure 8.11: RF Feedback block diagram.

(DAC) with rms noise $z^{2}$, and the backend processing (summing junction and amplifiers) with rms noise $w^{2}$. Based on this model, the total noise power can be modeled as

$$
N^{2}=G_{\text {analog }}^{2} y^{2}+G_{\text {digital }}^{2}\left(x^{2}+z^{2}\right)+w^{2} .
$$

By terminating the input of the RF Feedback, switching the analog and/or digital path on and off, and adjusting the gains $G_{\text {analog }}$ and $G_{\text {digital }}$, it is possible to determine the contributions from each one of these four components. Table 8.3 presents the estimates from these measurements. It is obvious that the Digital and Analog

\begin{tabular}{|c|c|c|c|}
\hline Digital $(\mathrm{x})$ & Analog $(\mathrm{y})$ & DAC $(\mathrm{z})$ & Backend $(\mathrm{w})$ \\
\hline 2.2 & 3.6 & 0.25 & 0.28 \\
\hline
\end{tabular}

Table 8.3: Component wise contribution in $\mathrm{mV}$ rms.

modules dominate the noise contributions. Figure 8.12 qualitatively confirms the measurements from Table 8.3. It shows the noise spectrum at the RF Feedback Output with different switch positions when the input is terminated. The noise level is significantly lower when both the analog and digital path are turned off. This figure also shows the $1 /$ f noise from the LLRF electronics. 


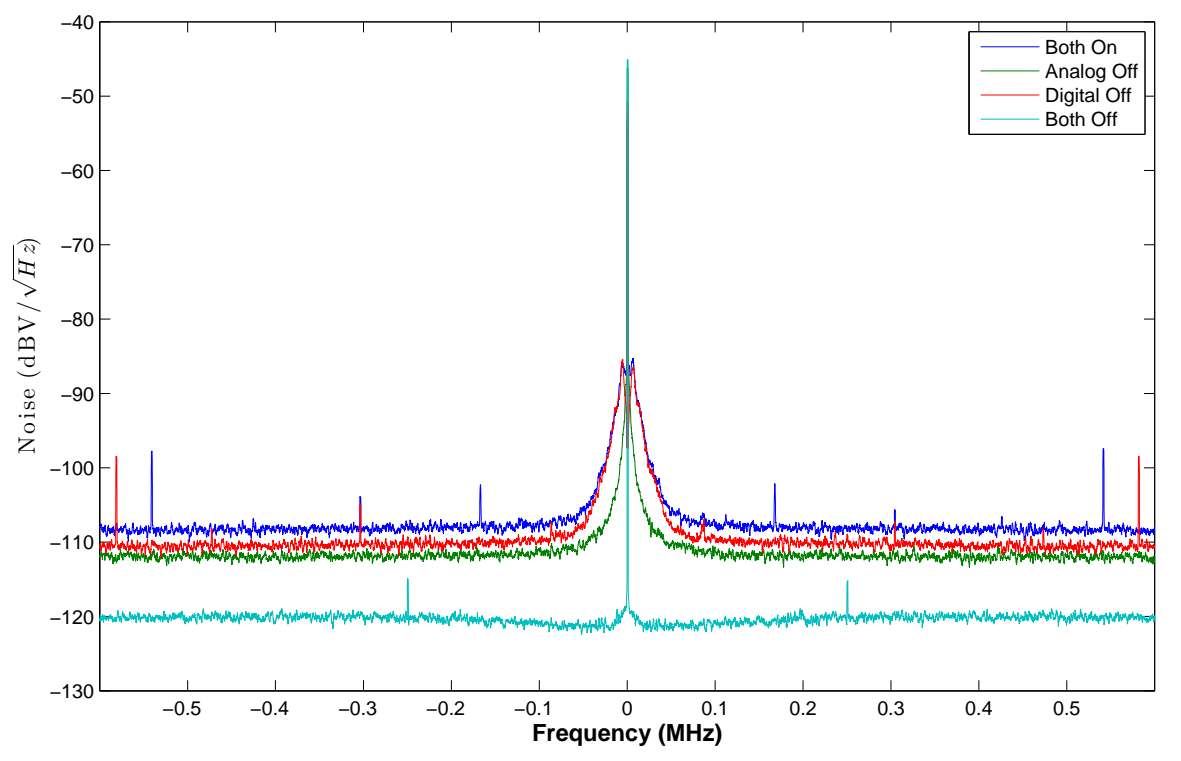

Figure 8.12: Noise at the RF Feedback Output with different switch positions.

Similar values were estimated by cascading the noise contributions of the electronics from the detailed layouts. This study also identified the most significant electronics components for the noise characteristics of each of the two dominant modules. The dominant components on the digital path are the differential amplifier driving the $\mathrm{ADC}$, and the digitizing noise of the ADC. For the analog path of the RF feedback the noise level is dominated by the large gain stage in the last stage of the analog demodulator ${ }^{3}$.

It is expected that as the controller gain is reduced, the noise levels would be initially reduced almost linearly with gain. As the gain gets lower though, the backend components would start dominating. This effect is visible in Figure 8.13 where the controller gain is set to $0,10,20$, and $31 \mathrm{~dB}$ and the RF Feedback input is terminated.

These studies provide a lot of insight on the performance of the LLRF system. If an alternative RF reference implementation is developed, the settings of the LLRF will strongly affect beam diffusion levels. Furthermore, this insight on the interplay between layout and component specifications will be very useful for noise budgeting

\footnotetext{
${ }^{3}$ Figure 8.1 shows a single demodulator, but in the actual implementation there are two similar systems, one for each path as shown in Figure 8.11.
} 


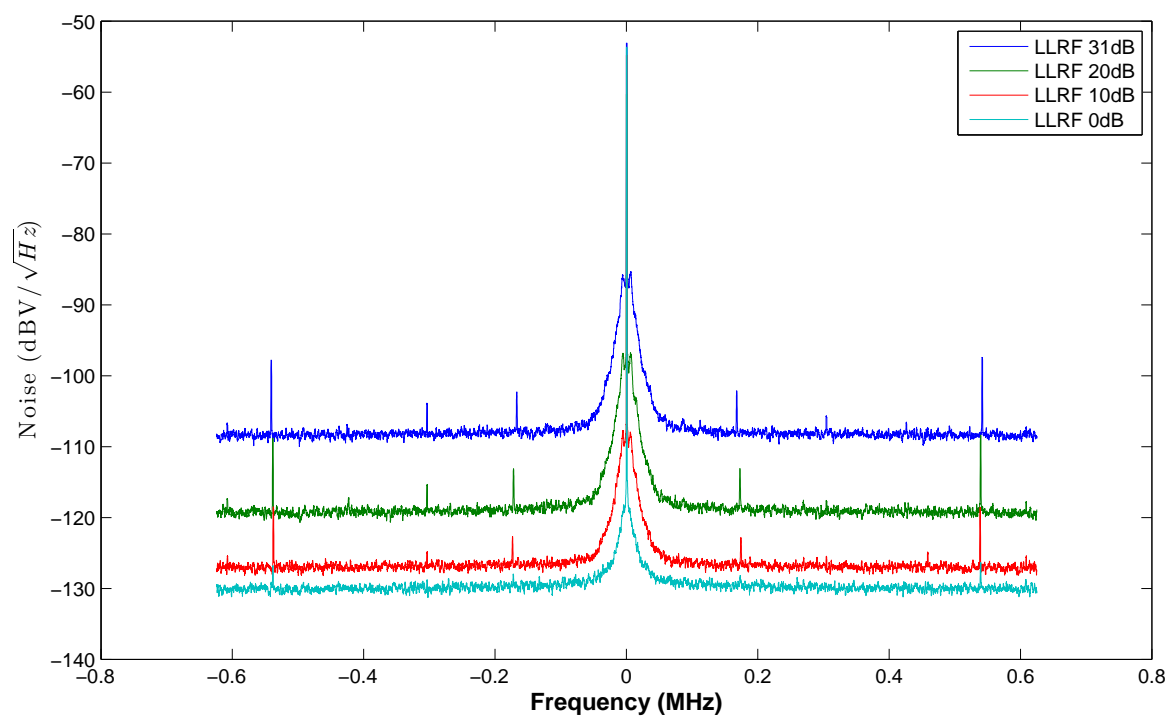

Figure 8.13: Noise at the RF Feedback Output as a function of controller gain.

in future LLRF systems.

\subsection{LLRF Feedback Noise Dependence on RF Feed- back Gain Settings}

The LHC time-domain simulations were initially validated through transfer function measurements [84]. These measurements though do not fully capture the dynamics performance of the system. Using the noise levels for the LLRF components presented in Section 8.4 in the simulation, it was possible to estimate the cavity phase noise spectrum due to the LLRF for various RF feedback gain settings and compare with measurements from the physical system. Figure 8.14 shows the clear dependence of the wideband noise spectrum on the LLRF gain settings, as well as the close agreement of the LHC simulations with the measurement for the various gain settings, covering the full scale of operation. Since the RF reference noise contribution is not modeled in the simulation, there is a discrepancy at very low frequencies when the reference is the dominating noise source. The bump at $250 \mathrm{kHz}$ is being investigated. 


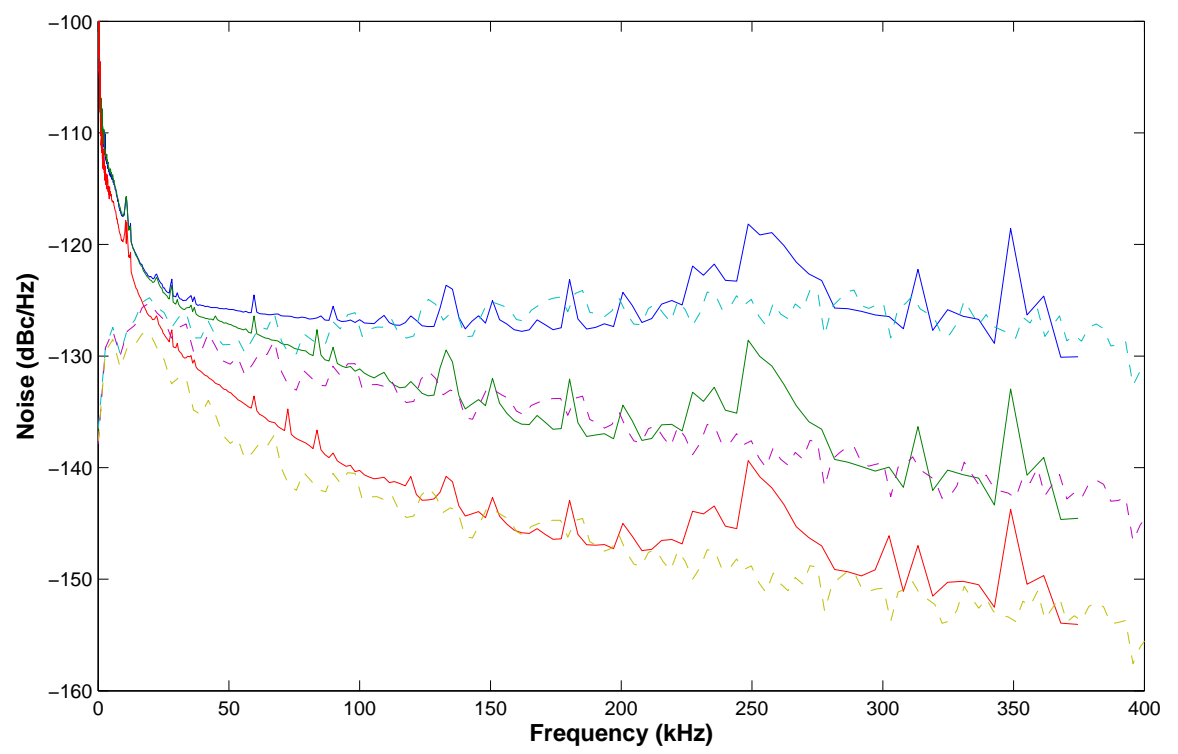

Figure 8.14: Cavity phase power spectral density with controller gain setting from 4B2 (solid lines) and simulation (dashed lines).

It is obvious from this figure that the simulation provides a good representation of the noise power spectrum of the RF cavity for frequencies higher than a few $\mathrm{kHz}$. As such, it can be very useful in predicting the system behavior for various RF configurations, estimating the coupled-bunch instabilities, and determining the contribution of the phase noise to beam diffusion around $f_{s}+k \cdot f_{\text {rev }}$ for $k>0$. 


\section{Chapter 9}

\section{Conclusions and Future Research Directions}

This dissertation focused on the RF station-beam dynamics interaction in circular accelerators through the use of time-domain simulations and models. These tools allowed the study of very different performance limiting beam dynamics effects for PEP-II and LHC.

Chapters 2 and 3 presented the background on beam dynamics and its interaction with the RF station. The longitudinal beam dynamics in circular accelerators is mainly defined by the beam current interaction with the accelerating RF stations. For stable operation, LLRF feedback systems are employed to reduce the fundamental cavity impedance and regulate the accelerating voltage. The LLRF operational choices have implications for the dynamics and stability of the both the RF systems and the particle beam.

The theoretical study of the RF station-beam interaction is difficult due to the complexity of the multiple feedback loops, the non-linear nature of the system, and the complicated multi-dimensional parameters space. A solely experimental approach would not only require a lot of machine time and suggest risks for system components, but also would not allow for an arbitrary variation of system parameters. Therefore, a time-domain simulation was developed to study and gain insight into this system, as described in Chapter 4. 
The time-domain simulation was first exploited to study longitudinal coupledbunch instabilities at PEP-II, a performance limiting effect. Studies of various operational choices, estimates of current limits, and the effectiveness of upgrades and alternative algorithms were presented in Chapter 5.

The similarity of the PEP-II and LHC RF architectures motivated the development of the LHC time-domain simulation, as shown in Chapter 6. The limiting beam dynamics effect at LHC though is quite different. The studies of the longitudinal beam diffusion at LHC in Chapter 7 increased the understanding of the phenomenon. Measurements presented in Chapter 8 helped identify the limiting RF component for machine performance.

These models and simulations can be readily adaptable to other accelerators, implementations, and beam dynamics. Examples of design studies for HOM driven instabilities and for PEP-X are included in the Appendix A and B .

\subsection{Main Contributions}

As part of this work, the following essential components for the study of the RF station-beam dynamics interaction were developed:

1. Theoretical formalisms and models that determine the longitudinal beam dynamics based on the LLRF implementation

2. Numeric time domain simulations that capture the dynamic behavior of the RF station-beam interaction allowing system studies without spending machine time

To validate these models and simulations, measurements were conducted at PEPII and LHC. Through this work, we gained great insight of the RF station-beam interaction which allowed us to implement upgrades leading to significant performance improvement and could help with the design of future systems, for the layout, noise allocation, and specification of technical components [41].

The PEP-II studies helped us develop new algorithms for impedance reduction (comb phase rotation), identify big impact upgrades (klystron driver amplifiers), 
achieve reliable machine operation, and finally reach a world record current of 3213 $\mathrm{mA}$ in the LER. Estimates of current limits due to operational considerations and coupled-bunch instabilities growth rates were computed for a variety of operational scenarios, helping plan the machine operations.

With the LHC simulation, models, and developed theoretical formalism, we estimated the effect of RF configurations, alternative designs, and next generation systems on the LHC longitudinal dynamics. Coupled-bunch instabilities were estimated and stability of the system up to nominal energies and currents is anticipated. The studies of the longitudinal beam diffusion helped identify the RF reference as the single performance limiting component. A major contribution was the development of a theoretical formalism relating the bunch length with the beam dynamics and the RF station configuration and noise sources, which was validated through LHC measurements and can help predict the effect of future technical implementations on beam diffusion. Finally, the developed RF station configuration and optimization tools were initially time saving and eventually imperative for LHC operations due to the no-access policies after the LHC incident of September 2008.

\subsection{Future Research Directions}

The RF station-beam dynamics interaction simulation, models, and theoretical formalisms have been used for extensive studies of two circular accelerators (PEP-II, LHC) and for design studies for the PEP-X light source. Studies of any possible configuration, new algorithm, or next generation system for the existing machines or for proposed future machines is possible with the tools developed in this dissertation.

These techniques and models were developed towards the end of the PEP-II operations and they were also helpful during the commissioning of the LHC. In both cases, it was not possible to change the design due to the timing of the contribution. It would be very interesting to see the influence of these models on the design of a future system.

In particular, estimates of the performance improvements and limitations of an all digital implementation of the LLRF could be very useful for future machines or LHC 
upgrades [85]. Noise allocation studies for such an implementation could provide a very useful tool for the next generation system designers. An all digital implementation is a more robust and flexible solution for the LLRF implementation while allowing for the addition of diagnostics and multi-user operation. A digital LLRF is also not susceptible to temperature drifts, channel crosstalk, and long transport distortion, contrary to an analog implementation. The longer latency, the sensitivity to clock jitter, and the complexity of programming though limit the digital implementation. But, compared to the total loop delay, the fractional delay increase of a digital implementation is not significant. For example, the total loop delay for the LHC is $600 \mathrm{~ns}$; the klystron alone contributes $130 \mathrm{~ns}$. The existing analog loop at the LHC introduces 15 ns of delay. Communication grade ADCs though have latency of only 35 to $60 \mathrm{~ns}$ [86], so the increase due to the ADC and the processing delay of the FPGA would not significantly increase the total loop delay. The digital implementation effect on performance could be studied with the existing models and simulations.

It would also be interesting to use the simulation to determine the system sensitivity to imperfections in the technical implementation, such as the channel crosstalk. Subsequently, a threshold amount or a budget for these imperfections could be estimated.

There are also many applications at LHC still to be explored. A methodology is currently being developed to inject noise at specific frequencies and with varying amplitudes at the LHC accelerating cavities. With this capability, it will be possible to better quantify the relationship between the RF noise and longitudinal emittance blowup, and in particular test the synchrotron frequency resonance and the effect of the aliasing due to the beam periodic sampling of the accelerating voltage.

Our earlier measurements identified the RF reference (Local Oscillator distribution) as the dominating component affecting the beam diffusion. Studies could be conducted to identify alternative technical LO implementations to reduce this effect and then evaluate their anticipated performance improvement with the developed tools.

A limitation of the beam diffusion theoretical formalism is the estimation of the 
equilibrium bunch length for each RF configuration. Even though the equilibrium bunch length is indicative of the bunch length growth rate, it would be helpful to improve the formalism to estimate the time evolution of the bunch length growth.

The coupled-bunch instabilities studies for the LHC were conducted at the nominal energy of $7 \mathrm{TeV}$. Since the updated LHC schedule calls for 2 years of operation at 3.5 $\mathrm{TeV}$, it would be useful to repeat these studies at the lower beam energy, in particular since the instabilities' growth rates are inversely proportional to the beam energy.

The particles at LHC are injected at $450 \mathrm{GeV}$ and then accelerated to the operational energy of 3.5 or $7 \mathrm{TeV}$. During this energy ramp, the synchrotron frequency crosses the $50 \mathrm{~Hz}$ ripple from the power supplies. Studies of the beam diffusion at this resonance could be very informative.

Finally, further features for the LHC RF station optimization and configuration tools could be added for future operations. In particular, the tools have not been tested on the 1-Turn Feedback, since the actual hardware has not yet been commissioned for all the RF stations. 


\section{Appendix A}

\section{PEP-II HOM Driven Instabilities}

The growth rates of the longitudinal higher-order impedance-driven beam modes have greatly increased since the initial PEP-II design and commissioning. This increase is attributed to the addition of $61.2 \mathrm{MW}$ RF stations with 8 accelerating cavities in the HER and $21.2 \mathrm{MW}$ RF stations with 4 accelerating cavities in the LER, which allowed operations at twice the design current and almost four times the luminosity. As a result, the damping requirements for the longitudinal feedback have greatly increased since the design, and the feedback filters and control schemes have evolved during PEP-II operations.

In this Chapter, growth and damping rate data for the HOM driven coupled-bunch modes are presented from various PEP-II runs and are compared with historical estimates during commissioning. The effect of noise in the feedback processing channel is also studied. Both the stability and performance limits of the system are analyzed.

\section{A.1 Longitudinal Instabilities in PEP-II}

The PEP-II rings have exhibited coupled-bunch longitudinal instabilities since commissioning. The longitudinal instabilities in PEP-II are driven by two impedance sources: cavity fundamental and cavity HOM. The cavity fundamental driven beam modes have been studied and predictions for higher currents, including studies of different configurations have been presented in Chapter 5 and [7], [67]. 
The LFB is a wideband bunch by bunch channel that addresses all modes via a digital control filter and broadband longitudinal kickers. The LFB is needed to control instabilities from the cavity HOM impedance [36]. It is a Digital Signal Processing (DSP) based flexible programmable system that can run FIR or IIR filters. A block

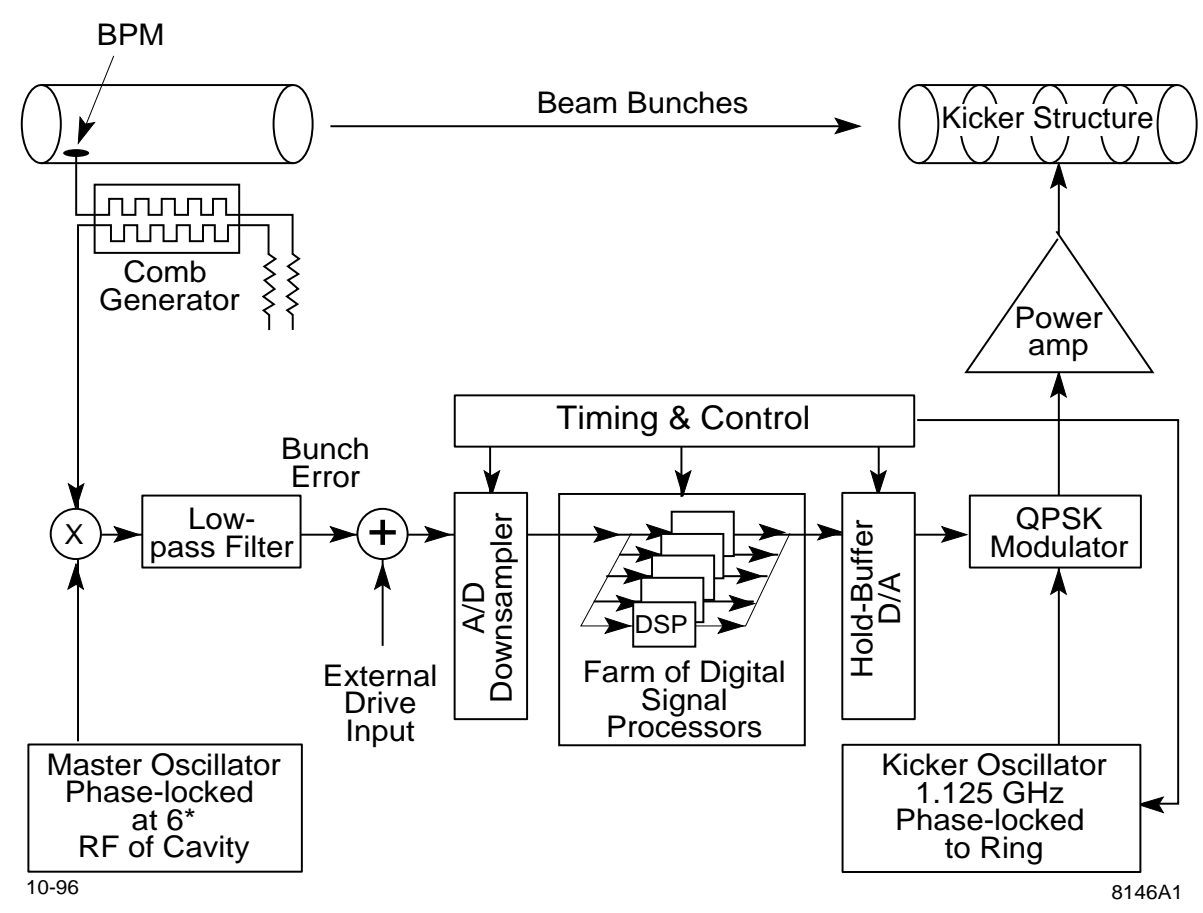

Figure A.1: Longitudinal Feedback System.

diagram of the LFB system is shown in Figure A.1.

The Beam Positioning Monitor (BPM) outputs an impulse for each bunch that excites the comb generator to produce a sinusoidal burst signal, with a frequency 6 times the RF frequency. This signal is mixed with the signal from the Master Oscillator. The mixer output signal passes through a low-pass filter that removes the higher sideband, and provides the phase error signal in a bunch by bunch basis. The A/D samples the phase error signal at a rate of $238 \mathrm{MHz}$. The LFB then processes the phase error bunch by bunch using an FIR filter. The filter output is converted to an analog signal that modulates in amplitude the QPSK modulated carrier signal. This composite signal is applied by the driving longitudinal kickers [36], providing the actual correction. 
Machine measurements in HER and LER are used in this Chapter to quantify the HOM driven growth rates, quantify the achieved performance of the broadband feedback, and highlight the performance limits in the systems as constructed.

\section{A.2 HOM-driven Modes: Growth and Damping Rates}

During the PEP-II design and commissioning, the impedance driving beam instabilities were estimated from cavity measurements [12] and were shown earlier in Figure 2.3. It is obvious from this figure that the cavity HOM vary significantly in amplitude and quality factor. Varied filling patterns can alias these HOM to different frequencies. The impedance estimates from the cavity measurements allowed calculations of the expected growth rates for the HOM driven coupled-bunch modes for an even fill [3], as shown in Figures A.2, A.3. The growth rates were calculated during commissioning for the design parameters of $1 \mathrm{~A}$ and 20 cavities for the HER and 2.25 A and 4 cavities for the LER [52], whereas the figures presented are at the operating currents at the end of PEP-II of $2 \mathrm{~A}$ for the HER and $3 \mathrm{~A}$ for the LER.

From these figures one can see that there are two bands that excite instabilities. The strongest is a $9 \mathrm{MHz}$ wide band that is aliased at around $105 \mathrm{MHz}$ (mode 770) from the $238 \mathrm{MHz}$ sampling and drives roughly 65 beam modes. The second band is a 7 $\mathrm{MHz}$ wide band that aliases at around $93 \mathrm{MHz}$ (mode 683). These wideband, instability driving impedances cannot be addressed by the LGDW system used for cavity fundamental driven modes, as they are outside the frequency range of the cavity and RF system. The LGDW system has roughly $5 \mathrm{MHz}$ of bandwidth (corresponding to approximately 37 modes), and cannot address the full span of 1746 modes which are controlled through the full bandwidth of the LFB system. Furthermore, it is impossible to tune those impedances using adjustments in water temperature or cavity tuners. The dominant impedance driving these instabilities is the cavity HOM impedance, which is proportional to the number of cavities. Therefore, the HOM driven coupled-bunch beam growth rates are proportional to the number of cavities. 


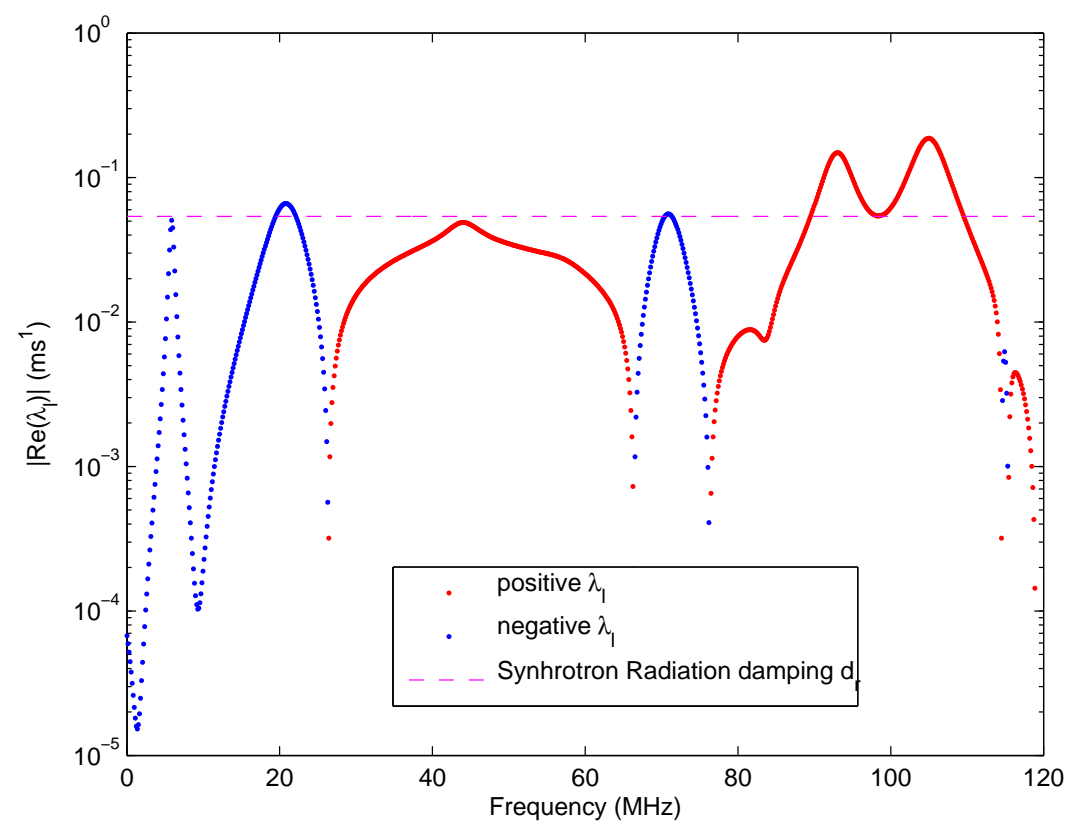

Figure A.2: Growth Rate Estimates from Impedance Measurements for 2 A HER. Two groups of unstable modes are expected in the 90 to $96 \mathrm{MHz}$ and 100 to $110 \mathrm{MHz}$ bands.

The beam growth rates are also proportional to the beam current. The above estimates were conducted using the final parameters from PEP-II. Any data from the earlier days of PEP-II used in this Chapter has been scaled appropriately for the latest PEP-II currents and cavities.

At nominal current both rings exhibit coupled-bunch instabilities in the absence of the damping feedback systems. Therefore, to measure the beam growth rates, we open the LFB loop for a few milliseconds letting the unstable beam modes grow, and then turn it back on to recapture the beam [10], [4]. The time-domain data of the beam motion is transformed to a modal domain and fit versus time. The complex exponential fit provides an estimation of the modal growth rates and oscillation frequencies. This process allows the estimation of the fastest growing beam modes. Other techniques are possible to measure slower growing modes [33]. 


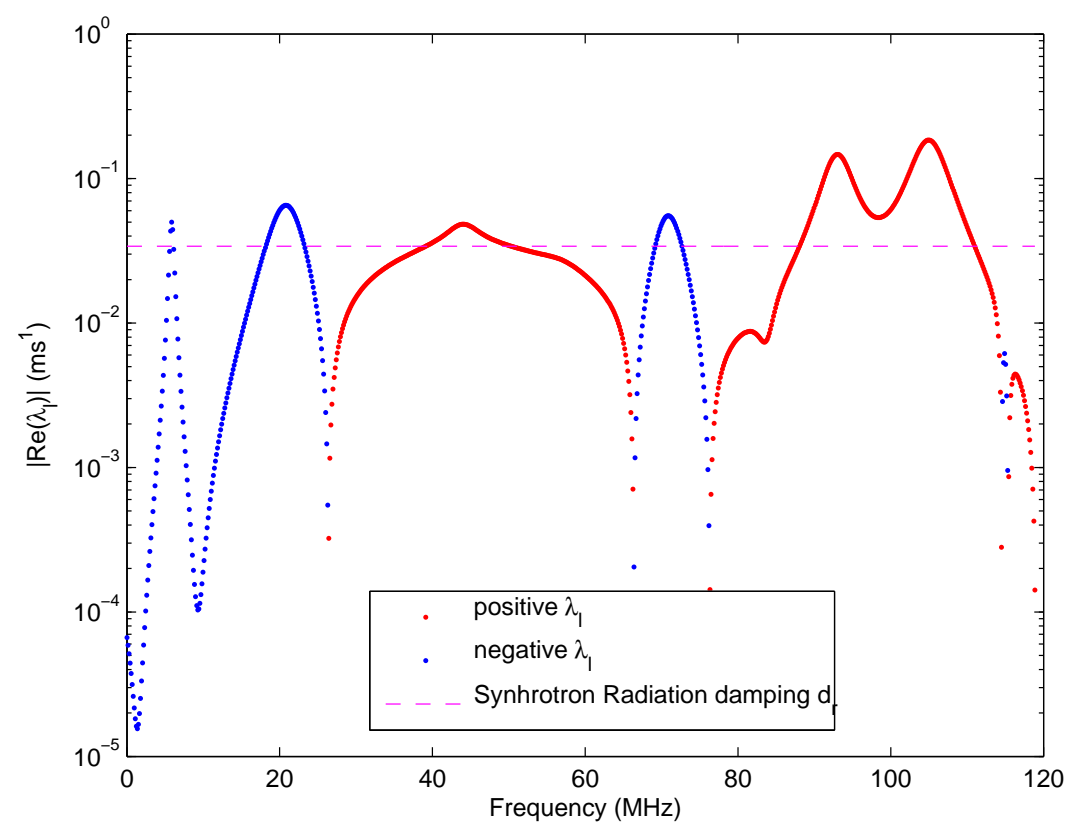

Figure A.3: Growth Rate Estimates from Impedance Measurements for 3 A LER. The two groups of unstable modes from Figure A.2 now compose a wider band from 88 to $112 \mathrm{MHz}$. A new band of unstable modes appears from 41 to $50 \mathrm{MHz}$.

The highest HOM beam growth rates and the corresponding damping rates reported below are from a band between mode 790 and 810 . This is very close to mode 770 that was estimated from the cavity data. The data reported are an average of the growth and damping rates over this mode range and multiple measurements.

\section{A.2.1 HER}

For the HER, the HOM driven coupled-bunch modal growth rates are shown in Figure A.4. Data points over 4 years of PEP-II operations are collected. The data points show great linearity with current as expected. To correctly compare these data points, the data from run 4 have been multiplied by 28/26 to account for the increase in the number of cavities. The black point has been appropriately scaled by a factor of $28 / 20$ from the early estimate based on the cavity design data. Even though this point was estimated with limited resources more than ten years ago, it 


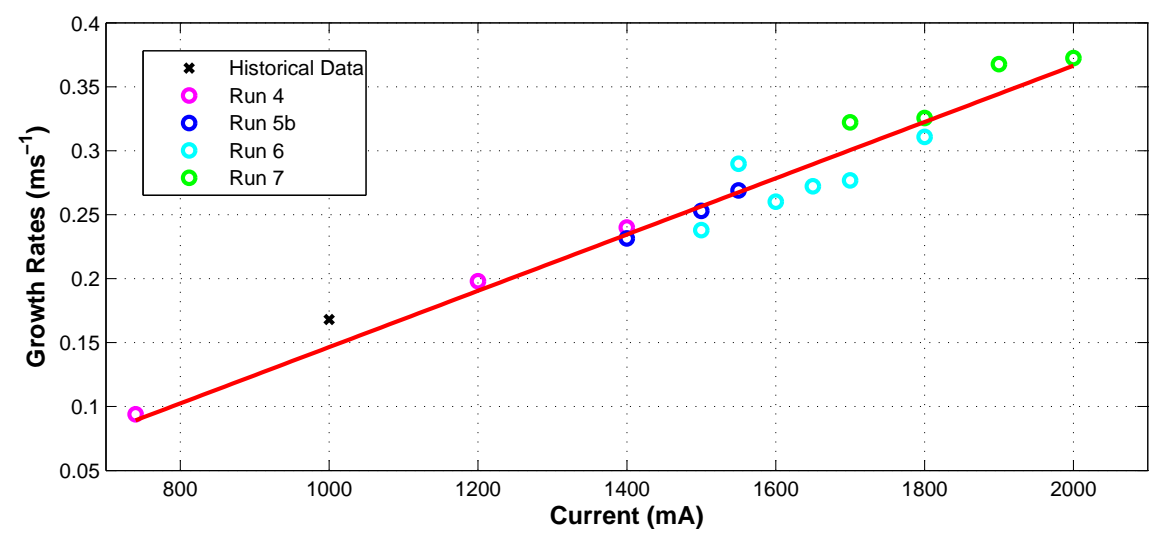

Figure A.4: HER HOM driven coupled-bunch modal growth rates for modes 790-810. shows great agreement with our data.

The damping rates for the higher currents are shown in Figure A.5. The LFB was

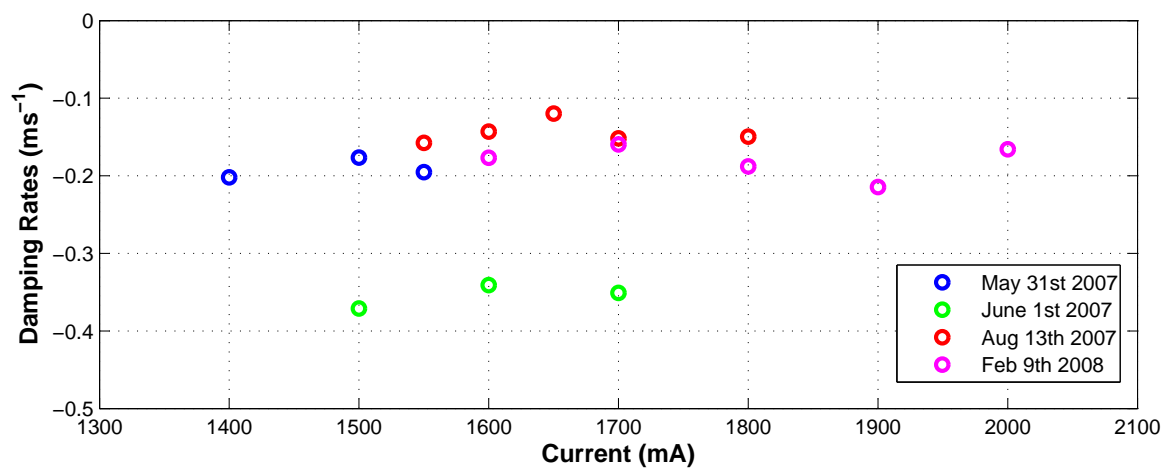

Figure A.5: HER HOM driven coupled-bunch modal damping rates (growth plus feedback damping).

configured with a 6 tap FIR filter centered around the $6 \mathrm{kHz}$ synchrotron frequency. The kicker and digital processing gains are constant for all the configurations shown. The filter gain is higher by a factor of 1.4 for the red and magenta data points. Even though the blue and green data points share the same configuration, they show a big difference in the modal damping rates. This difference is attributed to proper timing in the kicker. A 120 ps shift in the kicker timing reduces the gain by $3 \mathrm{~dB}$ and a 240 ps error drops the gain to zero [87]. Comparing the blue with the red and magenta data points, there is no improvement in the damping rate as expected 
with the higher filter gain due to the timing shifts. For the well-timed configurations, the damping margin was comparable to the measured growth rates, satisfying our margin criteria as defined in [7]. The lack of proportionality with current shows that the system is saturated. It should be noted though, that this saturation may be an artifact of the large longitudinal oscillations caused by the opening of the loop during our measurements. Even though this saturation is not experienced during closed loop operation, it signifies that this configuration approaches limited headroom from saturation limits.

Finally, Figure A.6 shows the synchrotron frequencies for the modal growth and damping rates with current. The two are in close agreement showing that the filter has been properly tuned for the system to introduce pure damping without affecting the modal oscillation frequency.

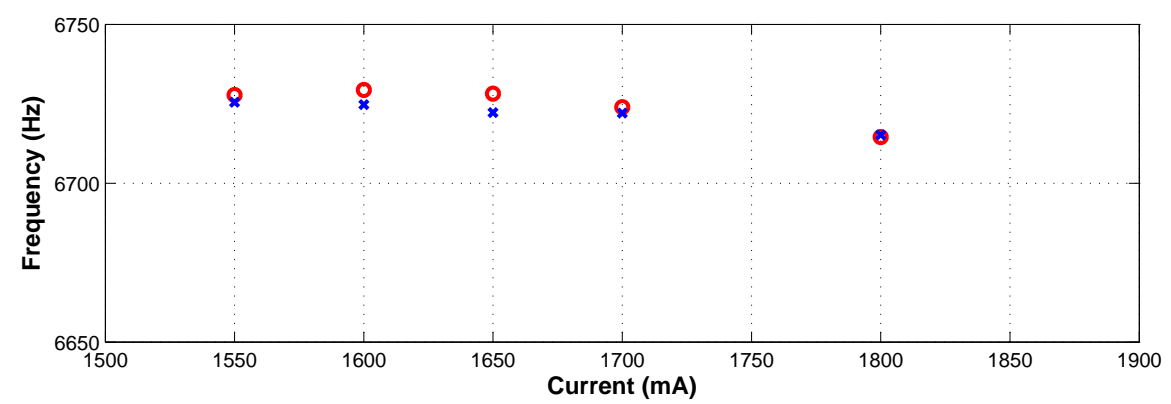

Figure A.6: HER HOM driven coupled-bunch modal synchrotron frequencies.

\section{A.2.2 LER}

For the LER, the HOM driven coupled-bunch modal growth rates are shown in Figure A.7. The data is from runs 6 and 7 and shows great linearity with current as expected. Similarly to the HER, the green point is from the estimate based on the cavity data using the LER design parameters of 2 stations and 4 cavities. The growth rate has been appropriately scaled by a factor of 2 for the increase of the number of cavities from 4 to 8 . Even though this point was estimated with limited resources more than ten years ago, it shows relative agreement with our data.

The corresponding modal damping rates are shown in Figure A.8. The LFB was 


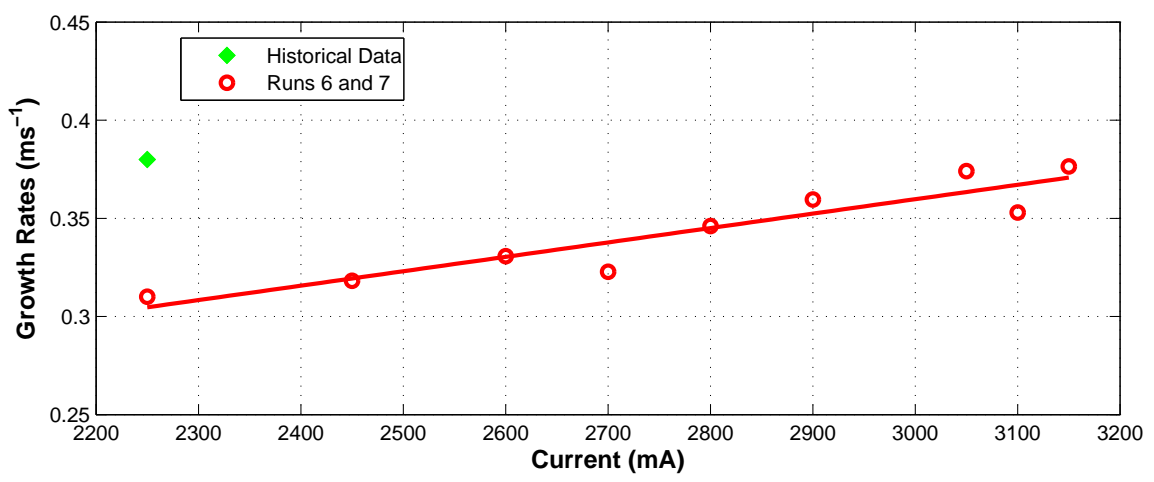

Figure A.7: LER HOM driven coupled-bunch modal growth rates for modes 790-810.

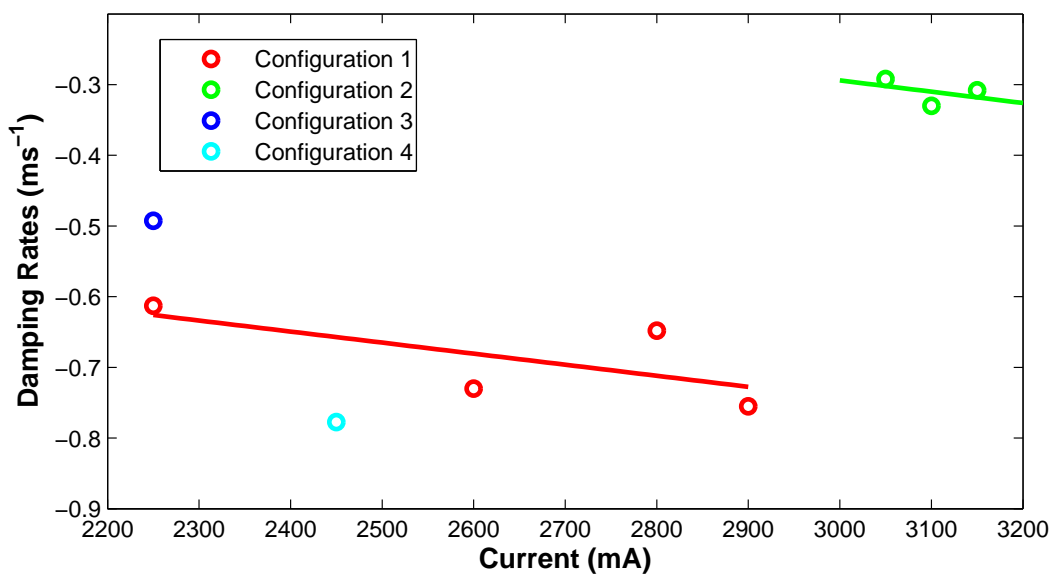

Figure A.8: LER HOM driven coupled-bunch modal damping rates (growth plus feedback damping).

configured with a 10 tap FIR filter centered around the $4 \mathrm{kHz}$ synchrotron frequency. The green and red data points share the same configuration. The filter gain of the blue data point is lower by a factor of 0.7 , whereas the filter gain for the cyan data is higher by a factor of 1.4 from the green and red points. The red line is fitted to the red data points. As described in the HER case, the big difference between the red and green data points could be attributed to timing issues. When the system is correctly timed, the damping margin was comparable to the measured growth rates, satisfying our margin criteria. The measurements agree with the expected behavior of increased damping rates with increasing gain and increasing current for the lower 
currents. The lack of direct proportionality though, signifies that the system might be saturated.

Finally, Figure A.9 shows the synchrotron frequencies for the growth and damping

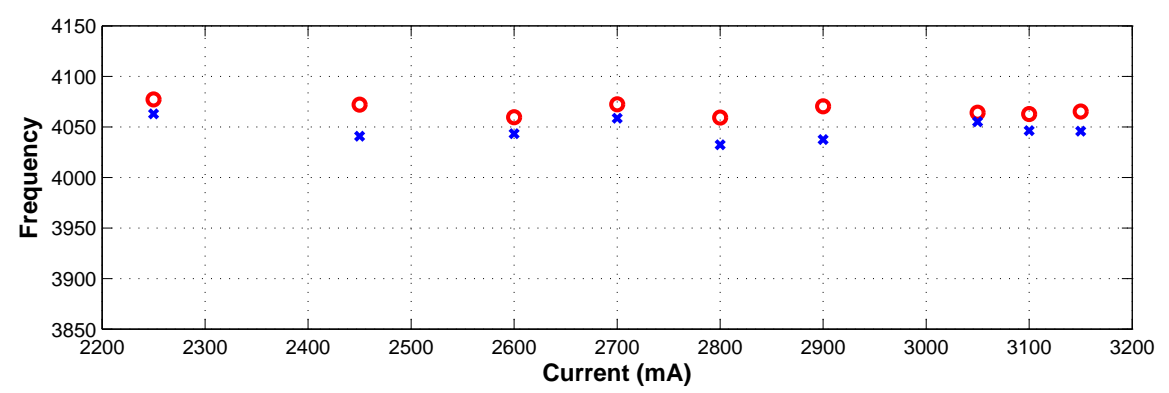

Figure A.9: LER HOM driven coupled-bunch modal synchrotron frequencies.

rates with current. The two are in close agreement as desired for the matched system described in the HER case.

\section{A.3 Feedback Model}

To understand the effect of the system parameters in the LFB stability and performance, an analysis based on a dynamic model is presented. The model includes the dynamics of the multi-bunch beam, the loop filter, and the perturbing noise sources that degrade the performance and stability of the closed loop.

The multibunch system is a multiple-input, multiple-output (MIMO) system. It can be represented in a simplified version as depicted in Figure A.10 [4], [88]. In absence of the LFB system damping the beam presents some unstable modes, defining an open loop unstable system. In addition, the system includes delays that in general limit the maximum open loop gain. The two main noise sources in the system can be grouped in the process noise $n_{p}(t)$ and the sensor noise $n_{s}(t)$. The process noise collects mainly all the perturbations introduced by the RF stations, kicker amplifiers, high voltage power supplies, and more. The sensor noise describes mainly the collective effects of the noise in the receiver. This noise has sources in the mixer, A/D 


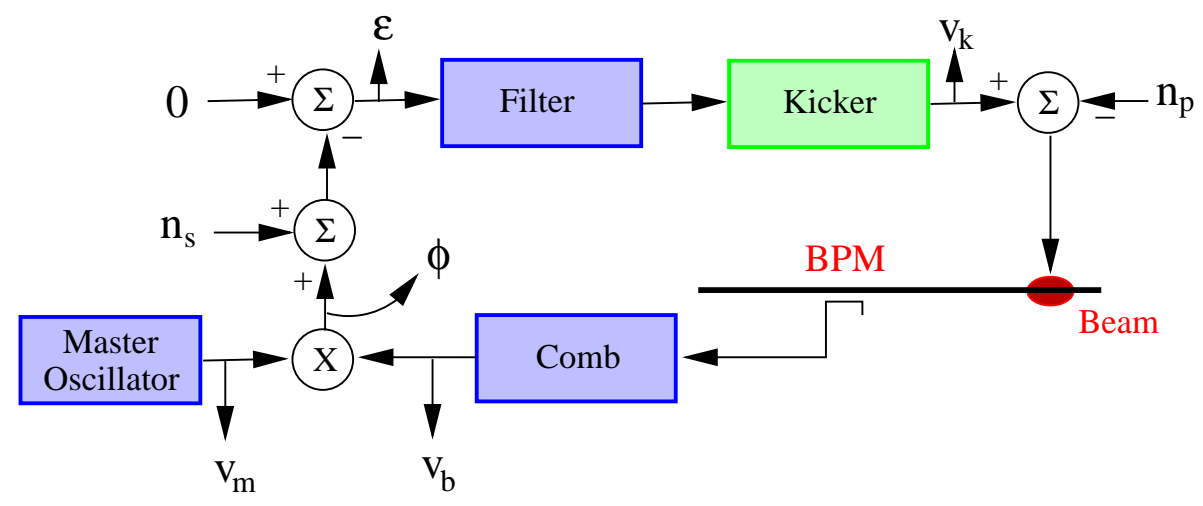

Figure A.10: Simplified LFB Block Diagram.

sampler, and cables connecting the BPM to the receiver. The phase noise in the Master Oscillator and the reference distribution are significant in the sensor noise. In a simple way, this noise can be analyzed assuming that the Master Oscillator produces a signal $v_{m}(t)=\left[V_{m}+n_{m}(t)\right] \cos \left(\omega_{0} t+\phi_{m}(t)\right)$, where $V_{m}$ is the amplitude of the sine wave, $\omega_{0}=6 \omega_{R F}, n_{m}(t)$ and $\phi_{m}(t)$ are the amplitude and phase noise, respectively. The Comb filter responds to the beam impulses measured by the BPM with a finite duration signal $v_{b}\left(t, I_{b}\right)=\left[V_{b}\left(I_{b}\right)+n_{b}(t)\right] \sin \left(\omega_{0} t+\phi(t)+\phi_{b}(t)\right)$, where $V_{b}\left(I_{b}\right)$ is the amplitude of the oscillation, proportional to the bunch intensity, $\phi(t)$ is the phase modulation in the beam produced by the noise $n_{p}(t)$ and the kicker signal, $n_{b}(t)$ and $\phi_{b}(t)$ are the amplitude and phase noise respectively, induced in the burst $v_{b}(t)$ by Johnson and pick-up noise.

The action of the mixer can be then simplified as $v_{b}\left(t, I_{b}\right) v_{m}(t)+n_{x}(t)$, where $n_{x}(t)$ is noise of the mixer at the output. Eliminating the upper sidebands at the mixer output by filtering, the detected signal is approximately $0.5\left[V_{b}\left(I_{b}\right) V_{m} \sin (\phi)\right]+$ $n_{A} \sin (\phi)+n_{\phi}+n_{x}$, where $n_{A}=0.5\left[V_{m} n_{b}+V_{b}\left(I_{b}\right) n_{m}\right]$ and $n_{\phi}=0.5 V_{b}\left(I_{b}\right) V_{m} \cos (\phi)\left[\phi_{b}+\right.$ $\left.\phi_{m}\right]$. To complete the model of the sensor noise $n_{s}(t)$, the ADC noise can be included giving $n_{s}(t)=n_{A} \sin (\phi)+n_{\phi}+n_{A D C}+n_{x}$.

In the absence of beam, the mixer noise is determined by the Master Oscillator and thermal noise in the RF and Oscillator signal paths, so that the amplitude of the signal is proportional to $V_{b} V_{m}+V_{m} n_{b}$ helping us determine the relative noise levels from the sensor, RF station and beam. In the presence of beam, the mixer noise is 
increasing non-linearly with current, making it harder to analyze. In the technical implementation in PEP-II though, the mixer noise is sufficiently smaller than the receiver noise and thus it is omitted in this analysis.

The beam dynamics of each bunch is modeled as a discrete harmonic oscillator driven periodically $\left(6 T_{\text {rev }}=45 \mu \mathrm{s}\right)$ by 6 equally spaced impulses with the equal amplitude. This represents the effect of kicking individually each bunch at the revolution frequency by a system that has a downsampling of 6. As it is depicted in Figure A.10, the detected phase of each bunch is perturbed by both the receiver noise $n_{s}(t)$ and the noise mainly coupled by the RF stations $n_{p}(t)$. Additionally, the destabilizing effect of the cavity HOM impedance is included in this model.

The set of transfer functions representing the ratio between the individual kicker signals $V_{k}(t)$ and the corresponding error signal $\epsilon(t)$ is defined mainly by the loop filter transfer function (FIR or IIR filters). This processing acts individually on the error signal generated by each bunch and generates a control signal $V_{k}(t)$ that kicks the same bunch a few turns later.

Mathematically, the system depicted in Figure A.10 can be analyzed in either the phase frame, where the phase $\varphi(t)$ represents the displacement of the individual bunches with respect to the synchrotron reference phase or in the modal frame, where the phase $\Phi(t)$ defines the oscillation of the different modes in the coupled multibunch system. The conversion between variables in these frames is defined by the NxN transformation $T$, where the $(m, l)$ element is defined by $T(m, l)=e^{-j 2 \pi \frac{m l}{N}}$. One advantage of the representation of the system in the modal frame is that the parameters defining the beam dynamic model for the unstable modes can be easily estimated form the growth rate measurements presented in the previous Section. Additionally, for the bank filter structure used in the LFB, where each filter processes the signal of an individual bunch, the transfer function of the filter is invariant with respect to the transformation.

The filter bank in the LFB is designed to stabilize the multibunch beam dynamics. There is a set of equal filters which stabilize the most unstable beam mode, and consequently all other beam modes. Based on this simplified criterion, the parameters of the loop filter can be selected to stabilize the beam. The design of the LER LFB 
loop filter follows. At $I_{b}=2250 \mathrm{~mA}$, from Figures A.7 and A.9, the eigenvalues of the dominant unstable mode are $\Lambda=\left(+0.3 \pm j 2 \pi 4\right.$.070) $\mathrm{ms}^{-1}$. The transfer function of the beam for that particular mode is shown in Figure A.11. For this system, a 10-tap

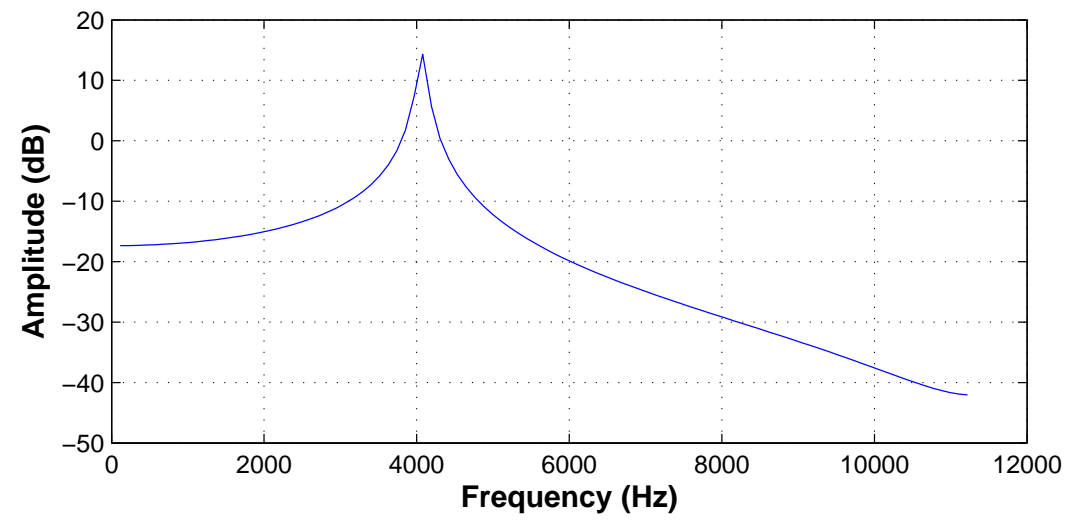

Figure A.11: Beam Transfer Function - Most unstable mode.

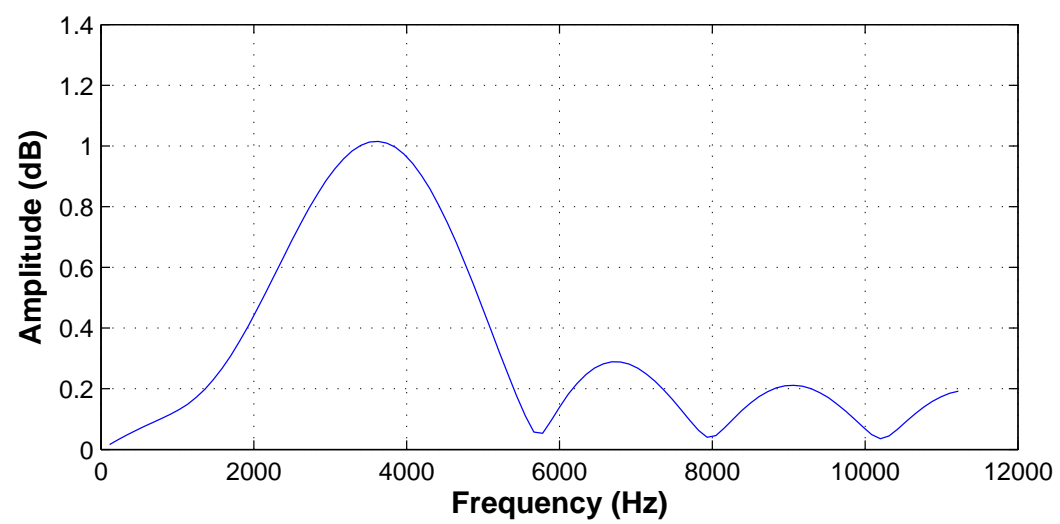

Figure A.12: 10-Tap Loop Filter Transfer Function.

FIR filter was designed. Its magnitude transfer function is depicted in Figure A.12.

When operating in closed loop, the eigenvalues of the composite system can be analyzed in the complex plane based on the Z-domain root locus analysis. Figure A.13 depicts the root locus for this particular system for open loop gains ranging from 0 to 3. Black squares show the location of the open loop eigenvalues of the composite system, as defined by the modal beam unstable eigenvalues (complex conjugated 


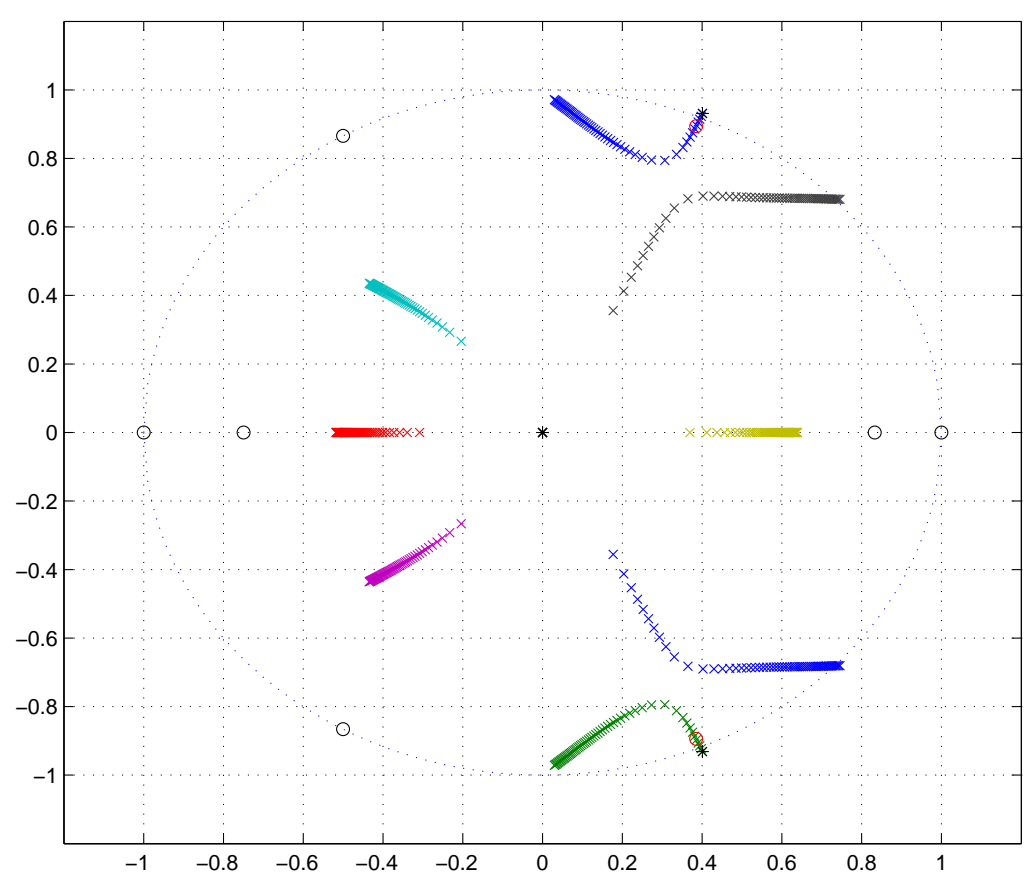

Figure A.13: Root Locus.

eigenvalues outside of the unity circle) and multiple eigenvalues defined by both the system and filter delays (located at the origin). The circles represent the zeros of the system defined mainly by the zeros of the filter. It is possible to evaluate the location of the closed loop eigenvalues for different gains for this particular beam mode. From Figures A.13 and A.14, it is possible to observe that there is a minimum gain to stabilize that particular beam mode, setting the closed-loop eigenvalues over the unity circle (open loop gain $=0.2$ ). Additional gain is necessary to set the closedloop eigenvalues to the red circle in the zoomed locus at $\Lambda=(-0.6 \pm j 2 \pi 4.070) \mathrm{ms}^{-1}$ (gain 0.5). This condition corresponds to Figure A.10. For open loop gain greater than 3 one observes that the system is unstable. This effect is mainly due to the delays in the system. In this particular system, the threshold gain is much higher that the gain used during operation, thus providing stability and good performance for the system. The maximum gain in the LFB system is actually limited by the amount of driven motion on the beam system originating from signals and noise in 


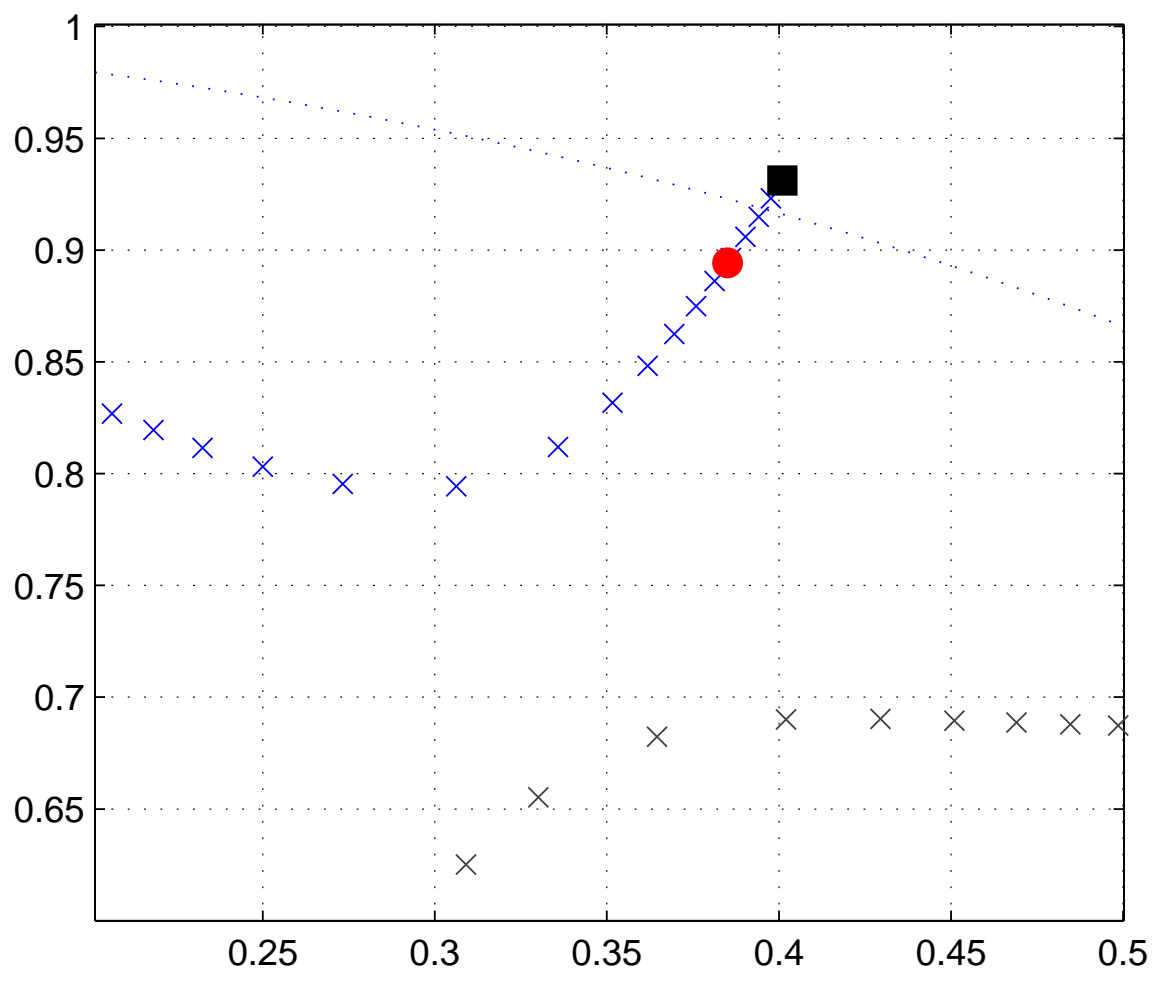

Figure A.14: Root Locus (zoom).

the RF stations and impressed on the beam via the RF cavities. These large driven motions could saturate the LFB power stages.

As mentioned earlier, the instabilities scale with beam current. But, the LFB gain also scales with beam current. So, if the system's gain $G(s)$ is above the threshold, the system should be stable for all currents. As can be seen in Figure A.13, this analysis breaks down for sufficiently high gains due to the system delay. The operating gain of approximately 0.15 though is significantly lower than the maximum gain value of 2.08 for stable operation, therefore, the maximum system gain set by the system delay does not present a significant system limitation. 


\section{A.4 Noise Floor Measurements}

In order to quantify the effect of the noise sources in the LFB system in both PEP-II rings, several noise measurement were performed using the built-in data acquisition system of the LFB. The downsampled signal digitized at 238 Msamples/sec by the ADC is recorded for offline analysis. This corresponds to the error signal $\epsilon(t)$ depicted in Figure A.10. The acquired signal is post-processed to calculate the noise spectrum. We estimate the power spectrum bunch by bunch and a quadrature average provides the equivalent noise spectrum of mode 0 in the modal domain.

To analyze the impact of the different noise sources, measurements were conducted in several configurations. Terminating the ADC input with 50 Ohms provides a measure of $n_{A D C}(t)$ - the quantizing noise in the $\mathrm{A} / \mathrm{D}$ and the noise in the internal sampler (as well as any systematic clock noise present in the processing). Turning off the gain in the Comb path at $3 \mathrm{GHz}$ measures the noise contribution from the baseband channel plus the noise contribution through the mixer and Master Oscillator. Finally, measuring the system in the nominal channel configuration but in the absence of beam quantifies the sensor noise $n_{s}(t)$ - the noise contribution from the whole RF path and processing channel (including any coherent pickup in cables, BPM, etc). It is important to notice that the noise source $n_{s}(t)$ defined in our model includes noise terms that are proportional to the amplitude of the Comb generator signal $V_{b}\left(I_{b}\right)$. The other two measurements mentioned above cannot completely quantify these noise terms since $V_{b}\left(I_{b}\right) \simeq 0$ in these cases.

Measuring the noise of the system with beam quantifies the impact of both noise sources $n_{s}(t)$ and $n_{p}(t)$ in Figure A.10. In closed loop the effect of these sources in the ADC is now affected by the transfer functions of the LFB system. These transfer functions will filter differently the perturbations due to the receiver noise $n_{s}(t)$ and the process noise $n_{p}(t)$. This effect can be estimated based on the model depicted in Figure A.12. Let us define the transfer function matrix in the Z-domain $F(z)$ as the ratio between the kicker signal $V_{k}(z)$ and the error signal $\epsilon(z)$ and the transfer function matrix $B(z)$ as the ratio between the detected phase $\varphi(z)$ and the kicking signal $V_{k}(z)$ in absence of noise. Then, in the phase frame the noise contribution 
in the error signal is $\epsilon(z)=[I+B(z) F(z)]^{-1}\left(n_{s}(z)+B(z) n_{p}(z)\right)$ with $z=e^{-j w T_{s}}$, $T_{s}=6 T_{r}=45 \mu \mathrm{sec}$.

\section{A.4.1 LER Noise Measurements}

Figure A.15 shows the noise levels for the four cases described above, measured with beam current at $3150 \mathrm{~mA}$ during run 7 . Label 'A/D' identifies the measurement of

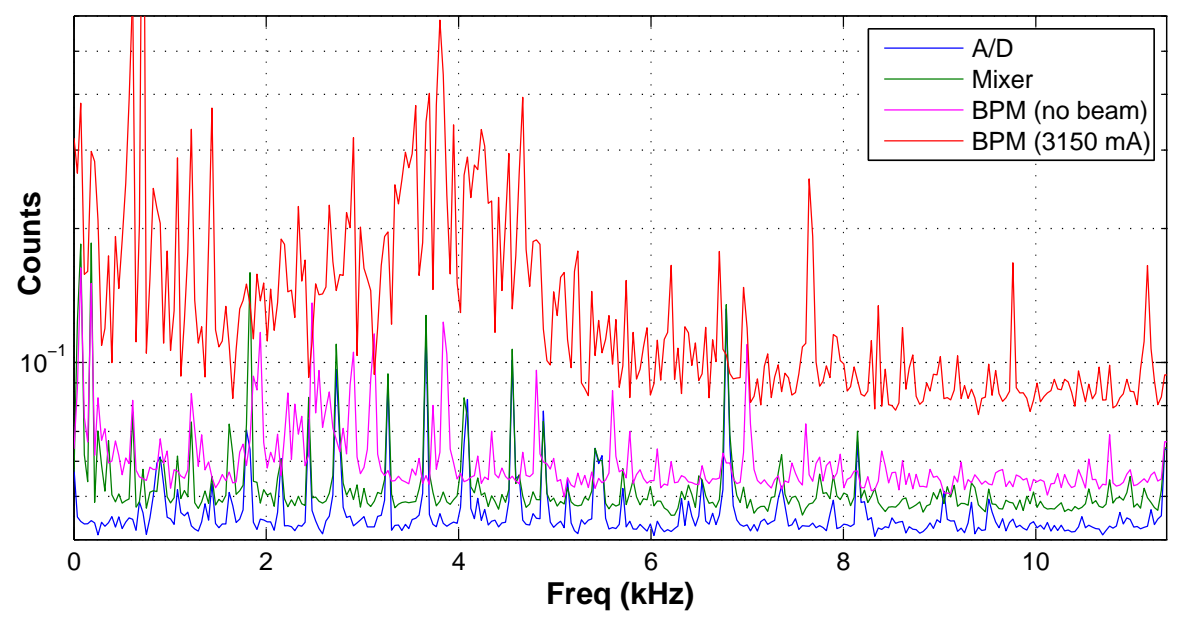

Figure A.15: LER noise measurements.

$n_{A D C}$, 'mixer' represents the case with the Comb gain turned off, 'BPM (no beam)' shows $n_{s}$, and 'BPM (3150 mA)' indicates the closed loop case with beam present. The measurements exhibit the expected order of noise power magnitude. For the LER, the noise in rms equivalent counts at the output of the ADC is approximately 0.66. To show the non-ideality and additional effects in the implemented A/D converter, a perfect quantizer, would have $0.32 \mathrm{rms}$ counts of quantization noise. It is important to observe that the beam noise is dominant and much greater than the other measurements. Part of the noise is amplified by the system around the synchrotron frequency at $4.07 \mathrm{kHz}$. The noise spectrum with beam shows the coherent motion of the beam at $720 \mathrm{~Hz}$ and $1440 \mathrm{~Hz}$, driven by the RF klystron High Voltage power supply ripple. Again, since in the presence of beam we have almost $2 \mathrm{rms}$ counts of noise, increasing the number of bits in the $\mathrm{A} / \mathrm{D}$ would have no effect on 
the system noise. A 6 bit A/D would still have a noise level comparable to the beam noise. To improve the system closed loop rms noise floor we either have to increase the available kicker power or to reduce the RF station noise (that drives the beam noise spectrum at low frequencies). Figure A.16 shows the lowest three measurements.

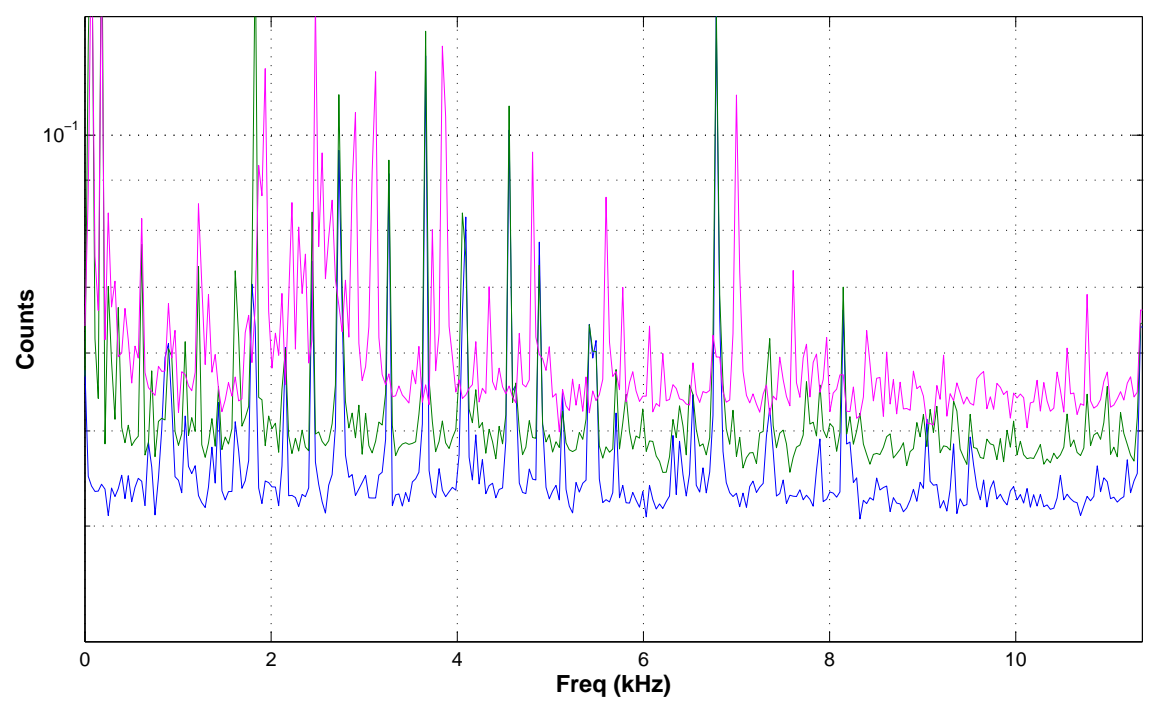

Figure A.16: LER noise measurement without beam.

The same conclusions can be reached from data from run 6 shown in Figure A.17, with $2700 \mathrm{~mA}$ beam current.

\section{A.4.2 HER Noise Measurements}

Figure A.18 shows measurements for the HER, exhibiting the same characteristics as the LER. We again see how the RF station noise impressed on the beam dominates the system. In the HER case the synchrotron frequency is close to $6 \mathrm{kHz}$ as can be inferred from the spectrum. 


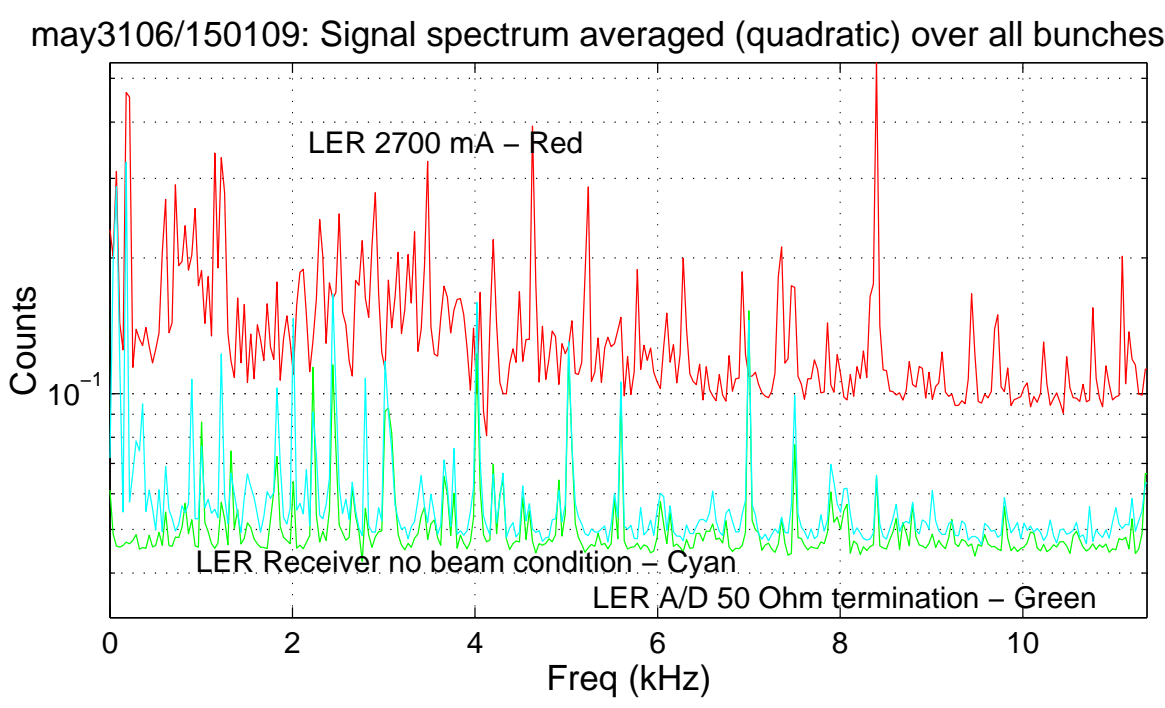

Figure A.17: LER noise measurements - run 6.

\section{A.5 Conclusions}

The LFB system was designed for much lower currents. Its programmable design allowed operations with much larger growth rates. Even though the system still had sufficient gain margin, the noise coupled to the beam from the RF station was very close to saturating the LFB at the highest beam currents. To operate at even higher currents, additional kicker power or improvements in the LLRF would have been necessary.

Another important limitation was the timing of the kickers. We see that timing shifts in the ps level reduced the system gain - and thus the stability and margin substantially. As a result, frequent inspection of the system was required. During run 6, the system was timed in the beginning (January 25 2007) and twice when the performance was largely degraded (March 20 and June 1 2007), with a great performance improvement.

It is possible that operation at higher currents could have reached the LFB system's thermal limits. The system's connectors, cables and loads have 2 to $4 \mathrm{~kW}$ power levels. Therefore, thermal limits, load power, circulator and amplifier reflected power should be carefully monitored in these conditions. 


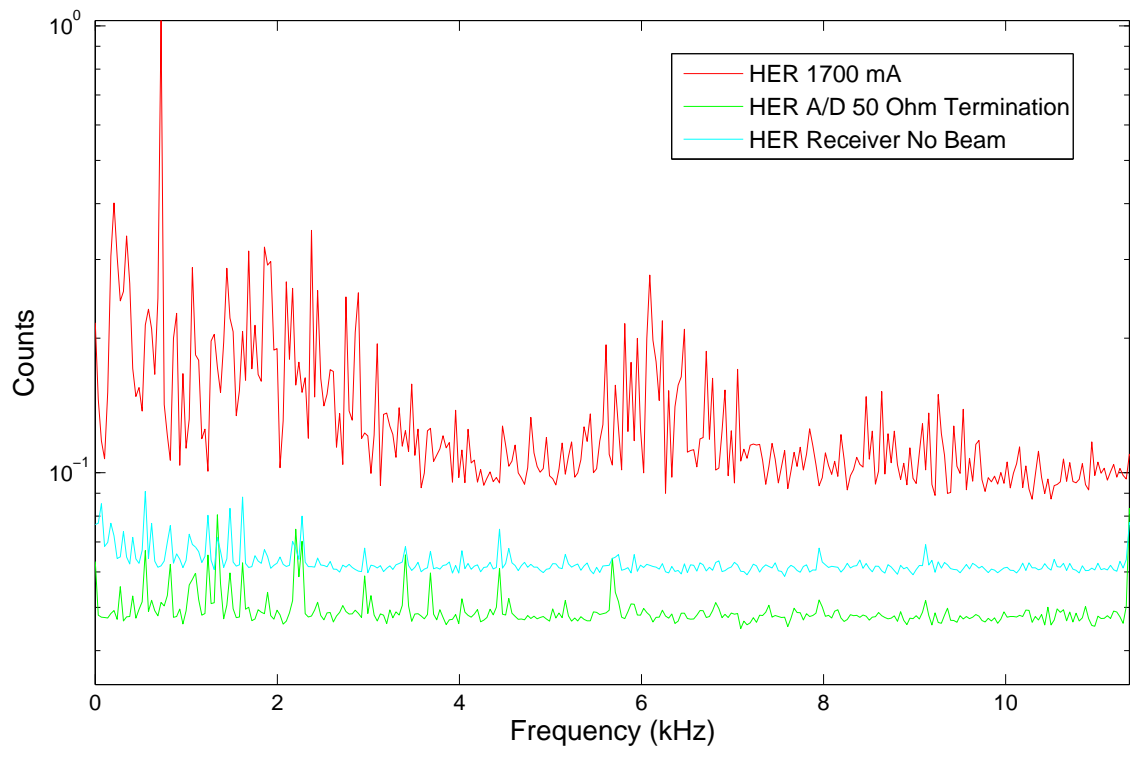

Figure A.18: HER noise measurements

The advantage of a programmable system like the LFB is that the filter configuration could be changed for different PEP-II operations. This can be necessary to optimize the system gain to provide the maximum possible instability control, but also avoid saturation. For example, since the group delay is not a limitation for this system, a filter with a higher number of taps could have been employed to reduce the saturation effects. 


\section{Appendix B}

\section{PEP-X Design Studies}

PEP-X is a proposed new light source which would reside in the existing PEP-II tunnel. This Chapter presents an estimate of the longitudinal motion and instabilities, with potential impacts for a synchrotron light source, as well as the potential effect of RF noise on the beam dynamics, as was presented in [89]. The PEP-X characteristics used in this work are those from the nominal design as defined in [90].

The interaction of the beam longitudinal dynamics with the fundamental and high-order mode (HOM) impedances of both RF systems is addressed to estimate the necessary stabilizing feedback systems. The coupled-bunch beam dynamics affected by the HOM cavity impedance are estimated based on the data measured/calculated during the design of the PEP-II cavities. The coupled-bunch beam dynamics defined by the fundamental and third-harmonic RF system impedance are estimated using the RF systems characteristics for a system based on the PEP-II normal-conducting cavities. This analysis shows that the longitudinal modal beam dynamics are more damped than the PEP-II modal dynamics, mainly because of the presence of wigglers in the machine, which significantly decrease the momentum compaction factor. This fact reduces the requirements for the feedback systems. The available feedback configurations are briefly discussed in this work.

The beam is also perturbed longitudinally by low frequency noise mainly coupled through the RF system. Our experience with PEP-II has shown that the klystron power supply ripple was a major source of perturbation on the longitudinal beam 
motion. For the PEP-X case this issue is more critical, since the spectrum of this low frequency perturbation is over the synchrotron frequency of the beam, increasing the coupling between the perturbation and the longitudinal beam motion. Solutions to reduce this beam motion are proposed based on feedback systems around the klystron in the RF stations. Further work will be necessary to fully quantify the effectiveness of each system on the PEP-X performance.

\section{B.1 Longitudinal Stability}

In this Section we estimate the longitudinal dynamics of the known cavity HOMs and the cavity fundamental, using the performance of the existing PEP-II impedancecontrolled LLRF topology as a metric. Issues of beam stability arising from fundamental cavity and HOM impedances are discussed via modal growth and damping rate estimates. The study is conducted assuming a double RF system consisting of powered RF cavities and passive third-harmonic cavities.

\section{B.1.1 HOM-driven Coupled-bunch Longitudinal Instabilities}

Following the analysis in Chapter 2, it is important to estimate the effective impedance driving the even-fill eigenmodes so that we can determine the HOM driven instabilities. During the PEP-II design, Bob Rimmer measured the center frequencies and quality factors for different HOM resonances in a prototype cavity, as shown in Table B.1 [12]. These measurements were validated with tests at PEP-II [3], so they were used with confidence to estimate the total PEP-X cavity impedance due to HOM resonances. These estimates result in the HOM impedance magnitude per cavity shown in Figure B.1.

To provide a reference point, the growth rates for both rings in PEP-II are briefly evaluated, and compared to the PEP-X scenario. Assuming the LER design configuration with 4 cavities (rather than the 8 of the final run) and the design parameters

$\alpha=1.2310^{-3}, I_{o}=2.25 A, E_{o}=3.1 \mathrm{GeV}, h=3492$, and $\omega_{s}=2 \pi 410^{3}$, Figure B.2 shows the absolute value of $\operatorname{Re}\left(\lambda_{l}\right)$ for each mode as defined in Equation 2.20 and 
Table B.1: Cavity Resonances

\begin{tabular}{|c|c|c|}
\hline$f_{r}(\mathrm{GHz})$ & $\mathrm{R} / \mathrm{Q}(\Omega)$ & $\mathrm{Q}$ \\
\hline 0.758 & 44.6 & 18 \\
1.009 & 0.43 & 128 \\
1.283 & 6.70 & 259 \\
1.295 & 10.3 & 222 \\
1.595 & 2.43 & 300 \\
1.710 & 0.44 & 320 \\
1.820 & 0.13 & 543 \\
1.898 & 0.17 & 2588 \\
2.121 & 1.82 & 338 \\
2.160 & 0.053 & 119 \\
2.265 & 0.064 & 1975 \\
\hline
\end{tabular}

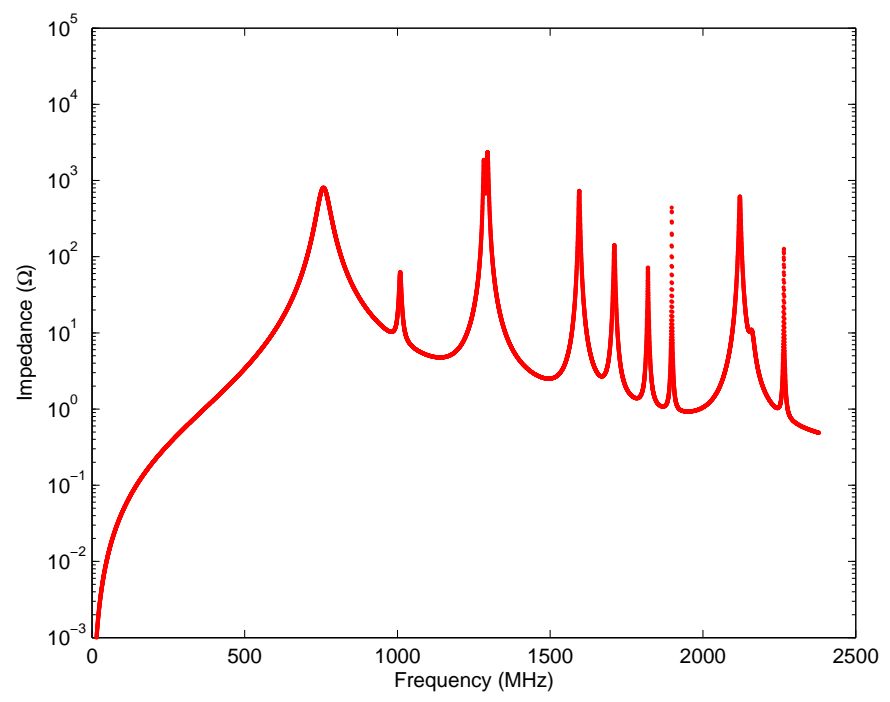

Figure B.1: PEP-II estimated HOM impedance per cavity.

considering a 'by-2' (every other bucket filled) beam filling pattern for PEP-II. Similarly, for the HER design configuration of 20 cavities (28 in the end of PEP-II) and $\alpha=2.4110^{-3}, I_{o}=1 \mathrm{~A}, E_{o}=9 \mathrm{GeV}, h=3492$, and $\omega_{s}=2 \pi 610^{3}$, Figure B.3 depicts the absolute value of $\operatorname{Re}\left(\lambda_{l}\right)$ for each mode. Effectively, the filling pattern determines the frequency band into which the HOM impedances are aliased. In these figures, we are plotting the absolute value of $\operatorname{Re}\left(\lambda_{l}\right)$ (red for positive and blue for negative) 


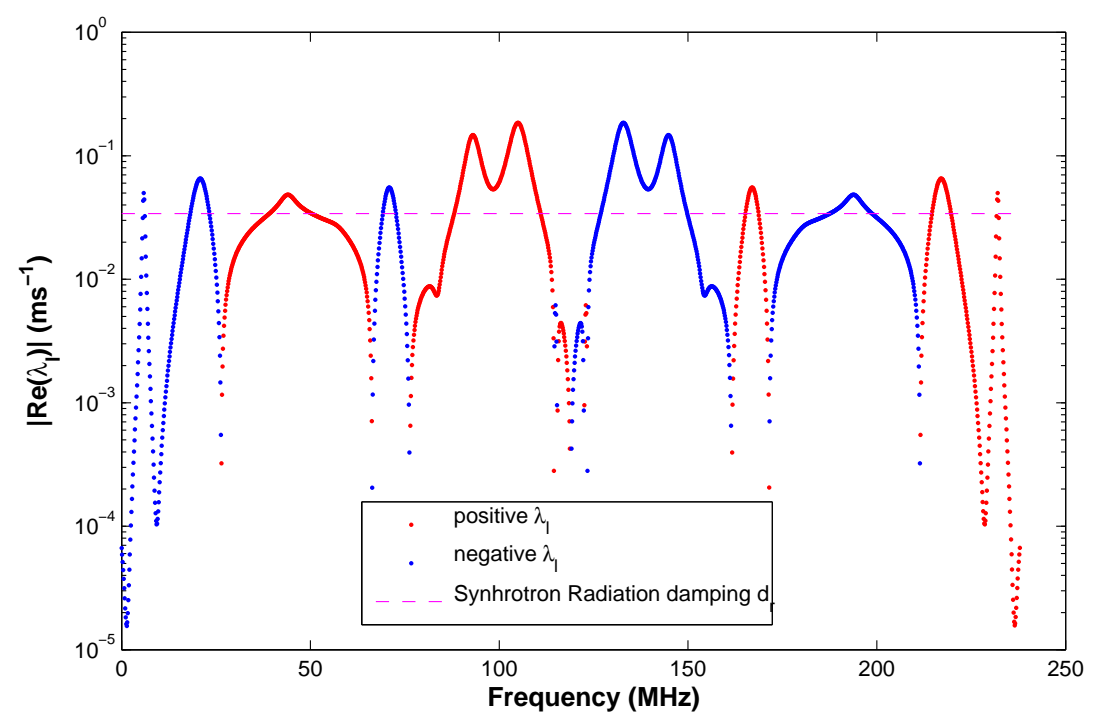

Figure B.2: PEP-II LER $\left|R e\left(\lambda_{l}\right)\right|$ for $I_{o}=3 \mathrm{~A}$ 'by-2' fill)

and comparing them with $d_{r}$ (horizontal line) to determine the HOM coupled-bunch stability. Unstable modes are those for which the red $\operatorname{Re}\left(\lambda_{l}\right)$ is greater than $d_{r}$. In PEP-II the strongest band of unstable modes was present in a region around mode $l=800$ that corresponds to a frequency of $108 \mathrm{MHz}$. This and other smaller unstable bands were assessed using a wide-band bunch-by-bunch longitudinal feedback system as discussed in Chapter A. During PEP-II runs, growth rates for modes around $l=800$ were measured at different beam currents, giving results very close to the one depicted in the figures.

Adapting the HOM impedance information to the PEP-X, it is possible to analyze the effects of the HOM impedances in the coupled-bunch stability. The total number of cavities estimated for PEP-X is 16 (PEP-II type cavities) and the beam filling pattern is 'by-1' (every bucket filled). The cavity impedance of the third-harmonic RF system implemented by either normal-conducting (NC) or super-conducting (SC) cavities is included in the calculations of the total impedance. It should be noted that only the fundamental resonance of the third-harmonic cavities is included, since there are no data available for other resonances. This omission has a negligible effect for the super-conducting case, but it could be noticeable for the normal-conducting 


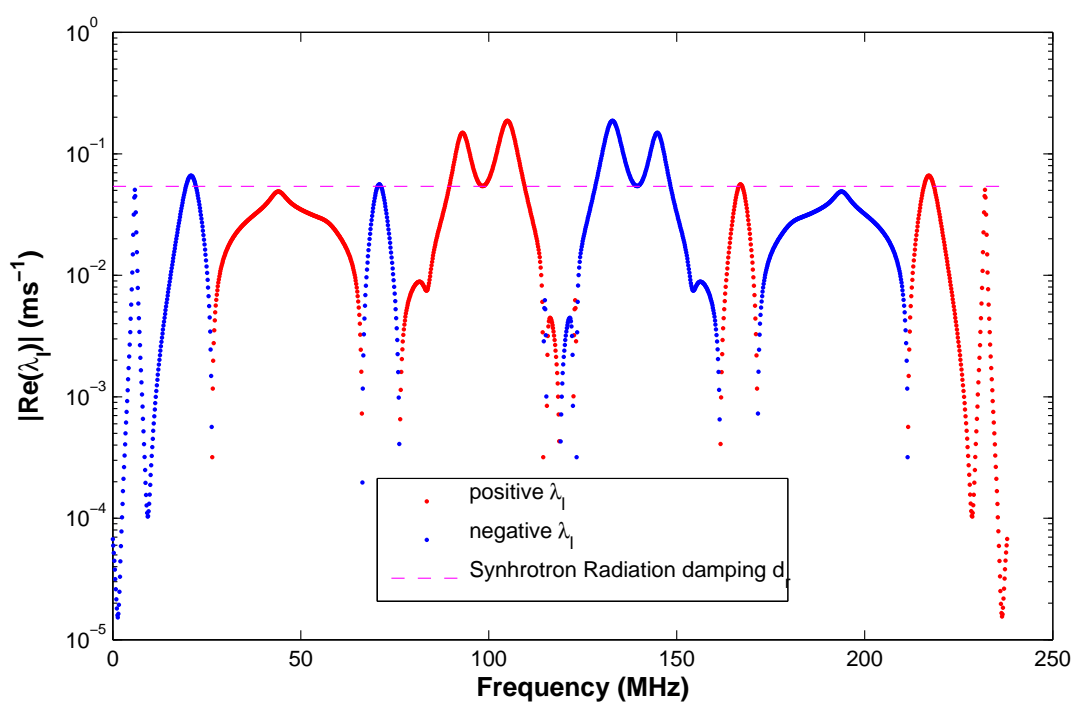

Figure B.3: PEP-II HER $\left|R e\left(\lambda_{l}\right)\right|$ for $I_{o}=2 \mathrm{~A}$ 'by-2' fill

case, depending on the final cavity parameters. The resonant frequency of the thirdharmonic cavities is at about $1.43 \mathrm{GHz}$ and the third-harmonic cavity parameters used in these calculations are the optimal parameters presented in [89]. Figures B.4 and B.5 depict the HOM impedance for the PEP-X case of 16 cavities taking into account the implementation of the third-harmonic system using 19 normal-conducting or 5 super-conducting third-harmonic cavities, respectively.

The estimate of $\operatorname{Re}\left(\lambda_{l}\right)$ for the PEP-X case is depicted in Figures B.6 and B.7, based on the impedance calculated above and the PEP-X parameters from [90]: $\omega_{R F}=2 \pi 476 \mathrm{MHz} ; \omega_{0}=8.5610^{5} \mathrm{rad} / \mathrm{sec} ; E_{o}=4.5 \mathrm{GeV}$, and $\sigma_{E} / E_{o}=1.1210^{-3}$. These estimates also assume a synchrotron frequency of about $500 \mathrm{~Hz}$ due to the third-harmonic cavities. From Figures B.6 and B.7 it is not expected that the HOM driven instabilities will be greater than the radiation damping for PEP-X. The reason for this improvement is two-fold. One obvious difference is the transition from the 'by-2' to the 'by-1' filling pattern. The PEP-X nominal filling pattern (every bucket filled) results in 3492 normal modes, spanning a $238 \mathrm{MHz}$ bandwidth. In comparison, the PEP-II 'by-2' filling pattern results in $1 / 2$ the number of normal modes 


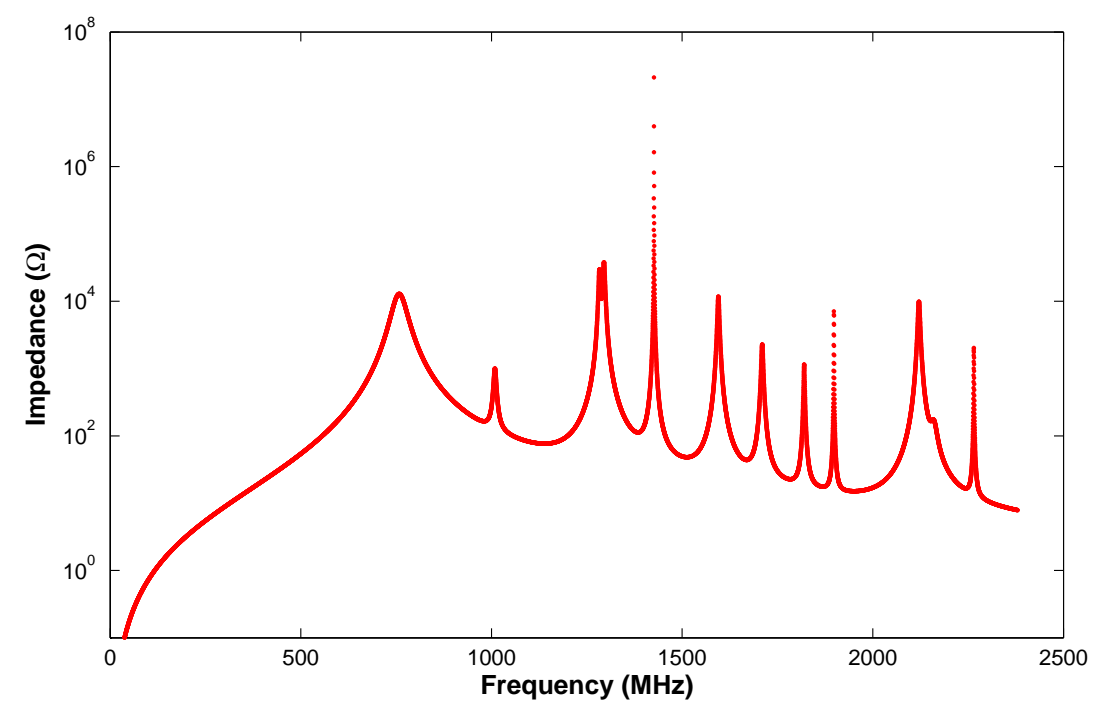

Figure B.4: Total fundamental cavity HOM impedance and third-harmonic normalconducting cavity impedance for PEP-X.

(1746), with a much narrower span of $119 \mathrm{MHz}$ for the folded impedances. As a result, the most unstable modes for the PEP-II case were in a band around mode 800, whereas in the PEP-X case this same impedance becomes a damped mode, while three bands of potentially unstable $\mathrm{HOM}$ driven modes at $60 \mathrm{MHz}, 160 \mathrm{MHz}$ and $225 \mathrm{MHz}$ can be now seen. Furthermore, while the growth rates are inversely proportional to the synchrotron frequency, the greatly reduced momentum compaction and increased radiation damping due to the wigglers, act to keep all the modes stable for a beam current of $1.5 \mathrm{~A}$. The wigglers reduce the momentum compaction factor to $\alpha=4.7210^{-5}$ for PEP-X, lower than $\alpha=2.4110^{-3}$ in PEP-II HER and $1.2310^{-3}$ in PEP-II LER.

On the other hand, one can see from Figures B.6, B.7 that the third-harmonic cavities alias on top of the fundamental impedance. With their resonance aliasing at $477.75 \mathrm{MHz}$ or $476.18 \mathrm{MHz}$ for the normal-conducting or super-conducting implementation, they affect a range of modes (wider range for the normal-conducting case) around mode 13 or mode 1 respectively driving unstable low-mode motion. Since this impedance is added on the fundamental - but is not reduced by the feedback loops - 


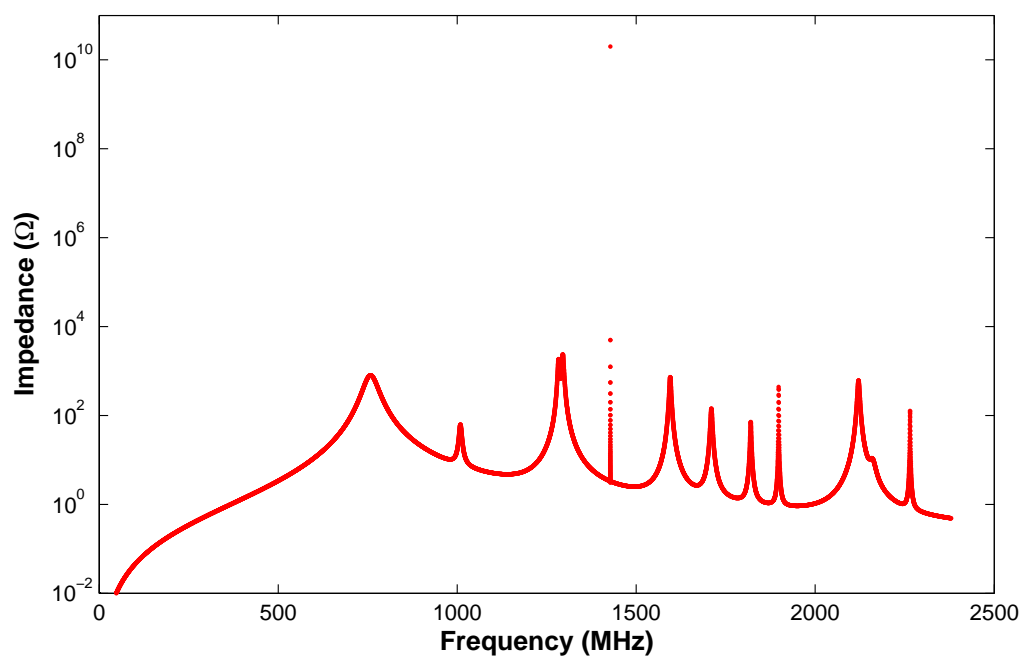

Figure B.5: Total fundamental cavity HOM impedance and third-harmonic superconducting cavity impedance for PEP-X.

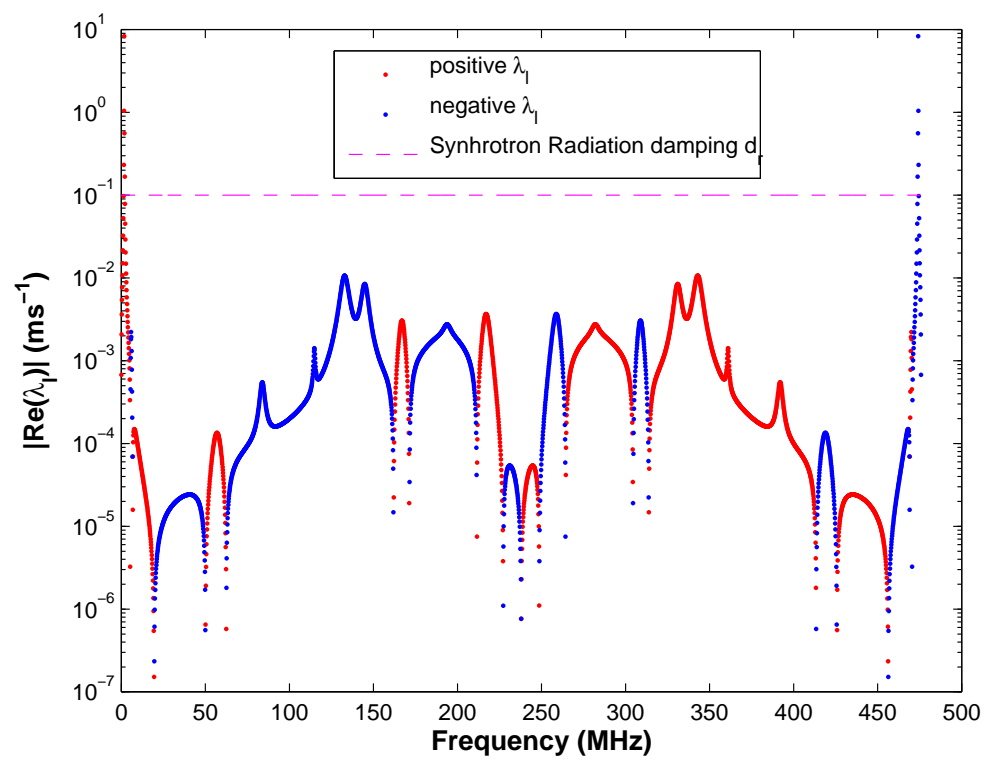

Figure B.6: PEP-X estimated $\left|R e\left(\lambda_{l}\right)\right|$, assuming 8 RF stations with 16 fundamental cavities, 19 normal-conducting third-harmonic cavities, $I_{o}=1.5 \mathrm{~A}$ and 'by-1' filling pattern. 


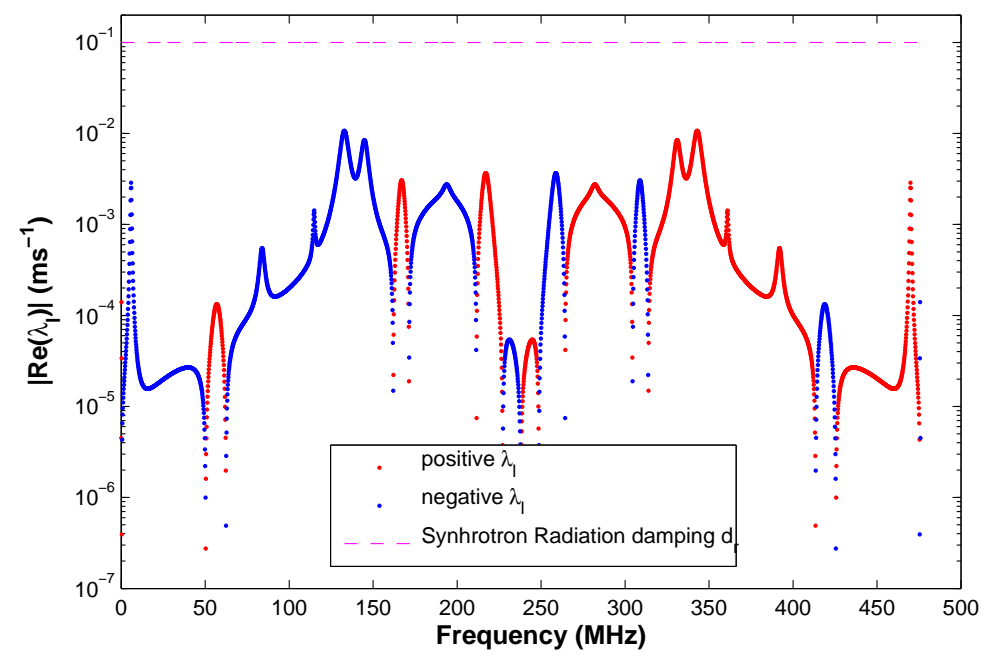

Figure B.7: PEP-X estimated $\left|R e\left(\lambda_{l}\right)\right|$, assuming 8 RF stations with 16 fundamental cavities, 5 super-conducting third-harmonic cavities, $I_{O}=1.5 \mathrm{~A}$ and 'by-1' filling pattern.

further development of our beam models and simulations are necessary to determine whether the existing damping systems will be sufficient to control this motion. A new damping mechanism might be necessary to counteract the instability due to the additional impedance from the third-harmonic cavity.

\section{B.1.2 Coupled-bunch Longitudinal Instabilities Driven by the Fundamental Mode and Third-harmonic System}

Our initial simulations of the cavity fundamental driven beam modes suggest that that low mode coupled-bunch instabilities driven by the cavity fundamental will force damping and RF feedback system requirements quite differently than those developed for PEP-II. In our analysis we are assuming that the RF systems employed will be consistent in achieved effective impedance with the systems used for PEP-II, even though they will likely not use the narrowband comb loop of the PEP-II design. It is also important to note that these studies do not include the effect of the harmonic cavity impedance on the cavity fundamental driven modes. 
For our initial studies we are using the macro-bunch and RF system simulation tools developed to study the PEP-II dynamics as shown in Chapters 4 and 5. In this study we model the low mode dynamics of the nominal PEP-X design, and we use the existing PEP-II Direct loop topology (but not a comb loop) and implementation for the RF system model. The narrowband comb loop is not particularly helpful in the PEP-X case, and the very low synchrotron frequencies, and large tune variation around the turn for the harmonic cavity cases are not consistent with the existing comb filter implementation.

From these studies, we expect the fastest growing beam mode driven by the detuned cavity fundamental to be approximately $0.4 \mathrm{~ms}^{-1}$, as can be seen in Figure B.8.
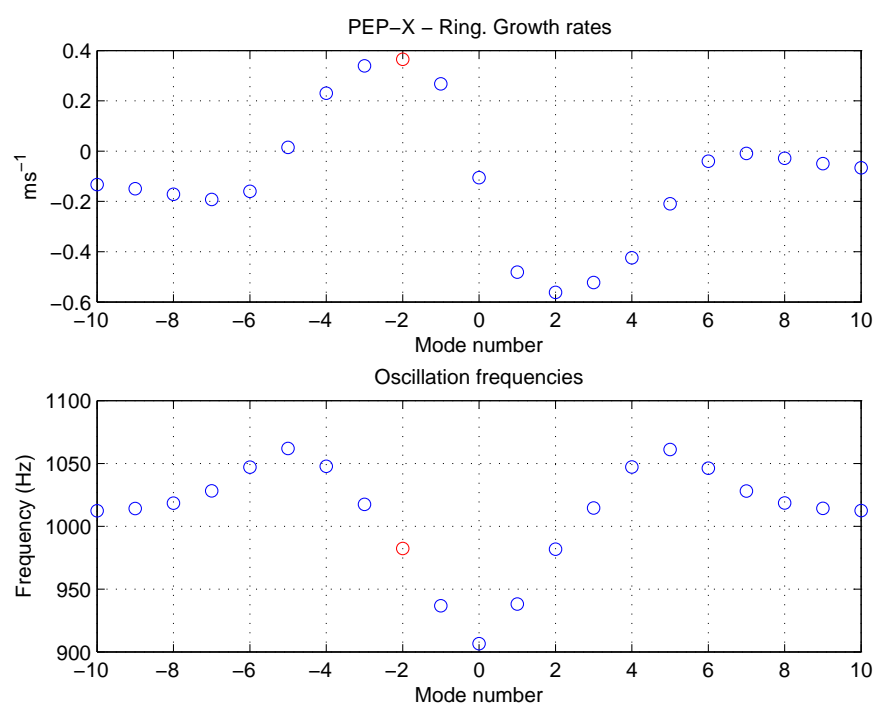

Figure B.8: PEP-X estimated growth rates with a direct feedback loop.

To determine stability, this growth rate has to be compared with the maximum achievable damping from the chosen feedback system. The accelerator parameters for PEP-X are much different than those in PEP-II, so the damping rates achieved in the past cannot be necessarily implemented. In PEP-II two active feedback systems were employed. The Longitudinal Feedback (LFB) was a wideband system that suppressed all modes. With consistent timing, it achieved damping rates in excess of $0.5 \mathrm{~ms}^{-1}$. The current implementation of the LFB is close to a possible design for PEP-X. The 
second system was the Low Group Delay Woofer (LGDW), a narrowband design that achieved much higher damping rates (up to $3 \mathrm{~ms}^{-1}$ ) for the modes near the cavity fundamental. Due to the significantly lower synchrotron frequency though, the PEP-X scenario is beyond the current capabilities of the LGDW. A different control algorithm would have to be implemented (possibly with the existing hardware), with a smaller number of taps in the designed filter.

\section{B.2 RF Station Noise}

While the beam may be free of HOM instability as shown in Subsection B.1.1. it will not be necessarily stationary longitudinally. We must estimate the amount of driven motion at the synchrotron frequency from noise mechanisms in the RF systems, master oscillator, etc. as well as the natural energy spread from quantum fluctuations. The synchrotron frequency for PEP-X is much lower than the $4 \mathrm{kHz}$ in the LER and the $6 \mathrm{kHz}$ in the HER, and it is in a band where the RF station noise is significant. As it was shown earlier for $V_{G A P}=13 \mathrm{MV}, \Rightarrow f_{s}=1.1654 \mathrm{kHz}$, for $V_{G A P}=10 \mathrm{MV}$, $\Rightarrow f_{s}=1.0089 \mathrm{kHz}$, and for $V_{G A P}=8 \mathrm{MV}, \Rightarrow f_{s}=885.2562 \mathrm{~Hz}$.

The significant reduction of the HOM and cavity fundamental driven coupledbunch instabilities for PEP-X in comparison with PEP-II is largely attributed to the significantly lower momentum compaction factor $\alpha$. The new lattice and the use of wigglers reduces $\alpha$ by almost two orders of magnitude. This reduction helps mitigate the effect of the lower synchrotron frequency on the impedance driven growth rates.

On the other hand, the reduced synchrotron frequency is a cause of concern due to the RF station noise. In Figure B.9 one can see noise measurements for the LER during PEP-II operations. The two "noisy" bands correspond to the beam (around the synchrotron frequency of $4 \mathrm{kHZ}$ ) and the RF station (around $720 \mathrm{~Hz}$ ). Since the estimated synchrotron frequency for PEP-X is at about $1 \mathrm{kHz}$ (or $500 \mathrm{~Hz}$ with a third harmonic cavity present), the effect of the station noise will be significant in exciting noise driven beam motion within the synchrotron resonance bandwidth. This is not "unstable" motion, instead the beam is simply excited from the noise power in the $\mathrm{RF}$ system and has an energy spectral distribution determined by the beam resonance 


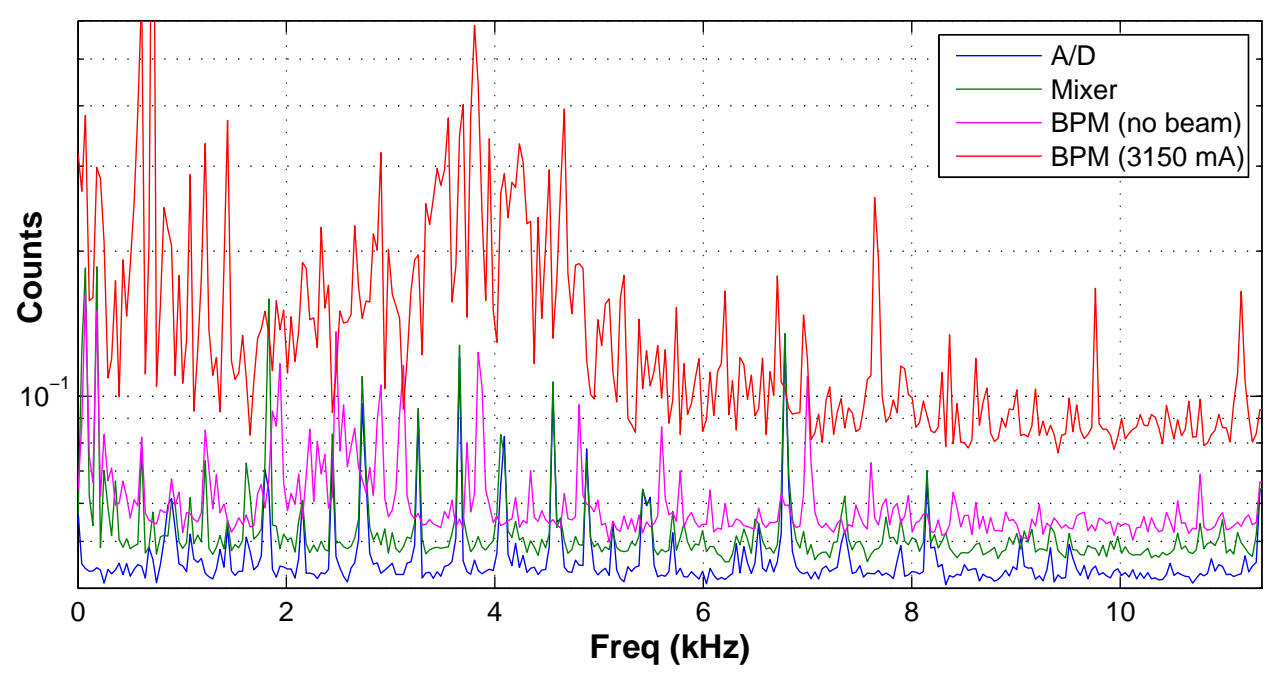

Figure B.9: PEP-II LER noise measurements

and the spectrum of RF station noise.

In PEP-II this noise was dominated by the RF Klystron high voltage power supply noise, and while the direct loop has some effect in reducing the cavity voltage variations the residual noise may still be of concern for PEP-X. As stated in [90], the longitudinal phase stability requirement has to be a small fraction of $1^{\circ}$ or equivalently the time-of-arrival jitter has to be less than 1 ps. Using data from PEP-II, we can estimate the expected longitudinal phase with respect to the synchronous particle $\Delta \phi$, and the time-of-arrival jitter $\Delta \tau$ due to the RF station noise.

Through a small signal analysis of the accelerating voltage, we model the longitudinal phase as

$$
\Delta \phi=\Delta \phi_{R F}+a(t) \tan \phi_{b}
$$

where $\Delta \phi_{R F}$ is the phase noise induced by the RF station, $\phi_{b}$ is the angle between the cavity voltage and the beam current, and $a(t)$ is the amplitude modulation of the gap voltage. We can approximate $a(t)$ by $\Delta V_{G} / V_{G A P}$ where $\Delta V_{G}$ is the effect of the station noise on the gap voltage. With this model and data from PEP-II, we can estimate $\Delta \phi$ for a PEP-X scenario to $0.1^{\circ}$ or equivalently $0.59 \mathrm{ps}$, assuming the $\mathrm{RF}$ station will present similar noise characteristics.

Even though this time-of-arrival jitter is smaller than the stability requirement set 
in [90], it only includes the effect of the RF station noise on the beam. Further effects in the transverse plane could possibly increase the jitter. It is also very important to note that this is an estimate based on PEP-II data. As mentioned earlier and shown in Figure B.9, the RF station noise is centered around $720 \mathrm{~Hz}$, so the effects on a PEP-X beam with synchrotron frequency at $500 \mathrm{~Hz}$ or $1 \mathrm{kHz}$, would be more pronounced, in which case this impact of RF driven noise must be better estimated and studied. A Klystron Linearizer, a technical direction from the PEP-II effort, might be applicable to control this high voltage power supply noise [53], [54]. 


\section{Appendix C}

\section{PEP-II Driver Amplifier Distortion Data}

The non-linear behavior of the PEP-II LLRF system $120 \mathrm{~W}$ solid-state driver amplifier was described in Section 5.5. The non-ideal amplifier response led to a direct increase of the coupled-bunch instabilities growth rates. In some operational scenarios, the growth rate increase reached a factor of four.

The amplifier functions were specified and tested for frequency response and gain uniformity in the initial development of the RF stations through large-signal transfer functions. These amplifiers and the klystron operate over a very large dynamic range though, since they deliver a large carrier at the RF frequency and small modulating signals around the revolution harmonics, as shown in Figure C.1. This is very different from communication applications or large-signal applications, and consequently a test of the third-order intercept point, a two tone test or a large signal transfer function would not be useful. Section 5.5 showed the ideal frequency sweep with a large carrier in Figure 5.12 and the non-linear response with a small modulation sweep in the presence of a large carrier in Figure 5.13 for one driver amplifier to show the significant variation in performance.

To more accurately characterize the small signal behavior a small signal transfer function was measured in the presence of a large carrier [56]. The carrier signal level is selected to be consistent with the high-power carrier in the actual system, while a 


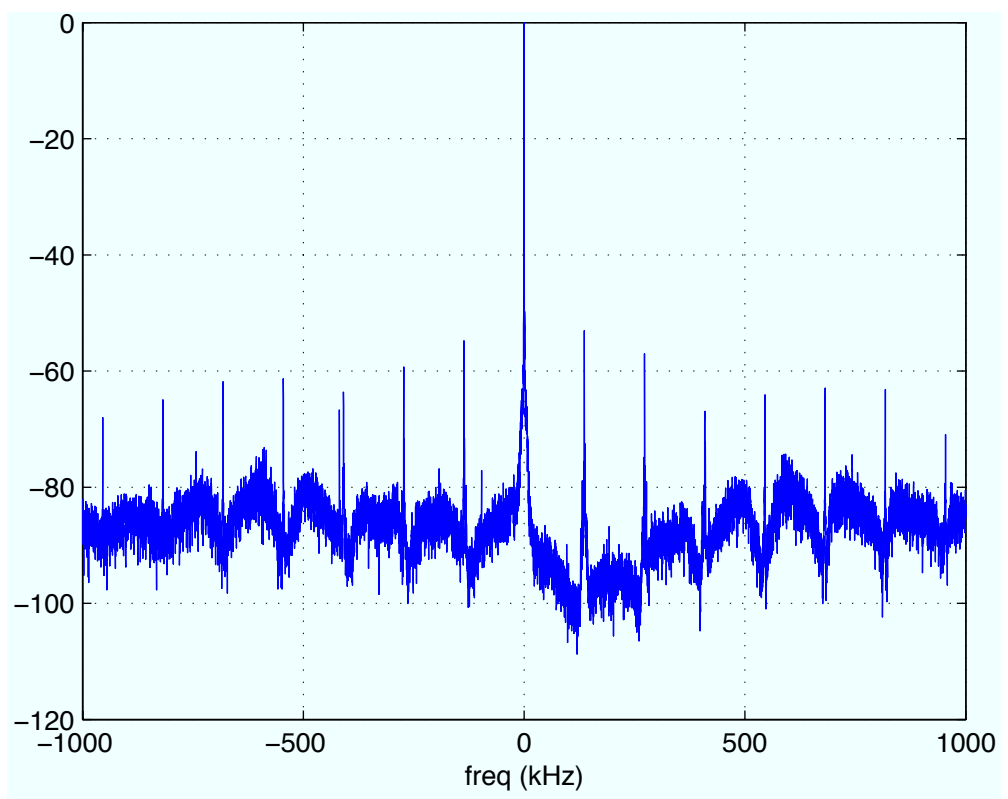

Figure C.1: PEP-II Klystron Output showing the large dynamic range of operation.

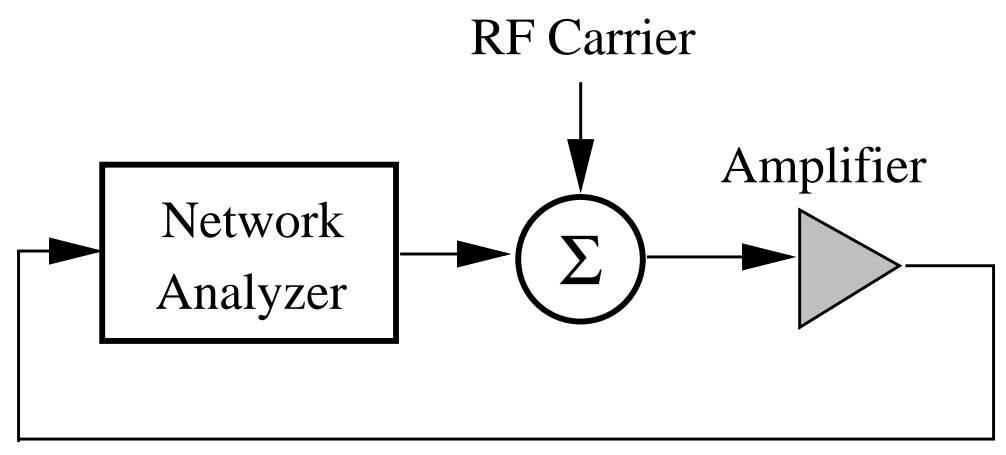

Figure C.2: Measuring network used to characterize the small signal behavior of the klystron driver amplifiers. 
network analyzer sweeps across the central operating frequency at levels well below the carrier.

In this Chapter, data from most of the installed driver amplifiers are presented. During the last two runs of PEP-II 11 RF stations were in operation for the HER and 4 for the LER. The HER stations were distributed as follows: two in region 4, three in region 8 , and six in region 12. All the LER stations were in region 4. Data from HER stations 4-1 and 12-5 are not included in this Chapter, because the klystrons were being replaced during the studies below. A driver amplifier from the Stanford Synchrotron Radiation Lightsource (SSRL) was also tested for comparison (SSRL and PEP-II share the same RF station architecture).

\section{C.0.1 Small Signal Transfer Function}

The first set of measurements were the small signal transfer functions of the driver amplifiers. Figures C.3 and C.4 show the small signal transfer functions in the presence of a 10 Watt large signal. Figure C.3 shows all the available amplifiers, whereas

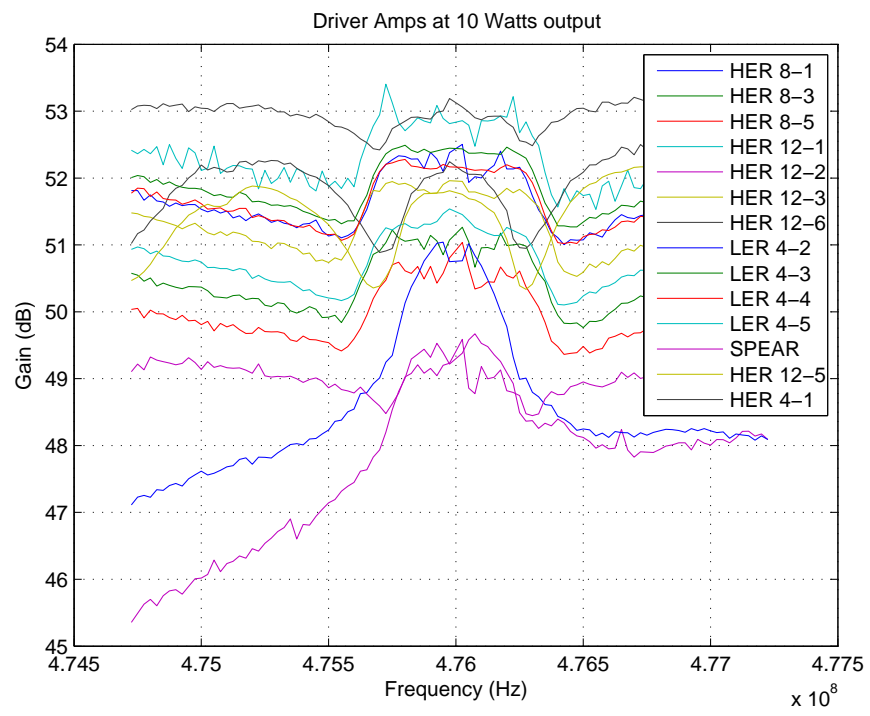

Figure C.3: Small Signal Transfer Function of PEP-II Driver Amplifiers at a largesignal power of 10 Watts.

Figure C.4 focuses on the worst ones: LER 4-2 and HER 4-1, 12-2, 12-5 and 12-6. 


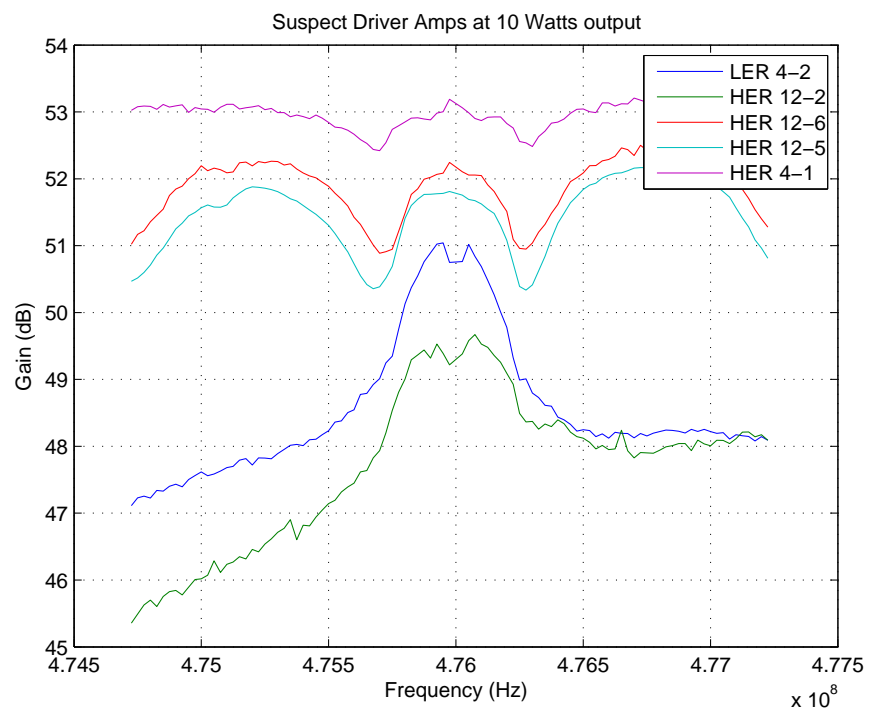

Figure C.4: Small Signal Transfer Function of PEP-II Driver Amplifiers at a largesignal power of 10 Watts.

Most of the amplifiers have less than a $1 \mathrm{~dB}$ distortion. HER 12-6, 12-5, 4-1 do not have a large deviation from the normal, but there is some unusual structure in the response. LER 4-2 and HER 12-2 are significantly worse. It is interesting to note that before the non-ideality of the driver amplifiers was discovered, there were a lot of issues with the tuning of these two RF stations, which were eventually explained by the amplifier distortion.

The small signal transfer functions for a 20 Watt large signal are shown in Figures C.5 and C.6 with the same conclusions.

\section{C.0.2 Intermodulation Plots}

If two or more signals are injected in a nonlinear system, the output includes a series of intermodulation terms. If the inputs are of frequencies $\omega_{1}$ and $\omega_{2}$, the output will contain frequencies at $n \omega_{1} \pm m \omega_{2}$. To quantify the degree of nonlinearity in these LLRF amplifiers, a swept carrier plus single-sideband image test was developed. Here a large signal carrier, and a swept upper sideband tone at a lower level are impressed at the amplifier input using a similar setup to Figure C.2. But instead of measuring 


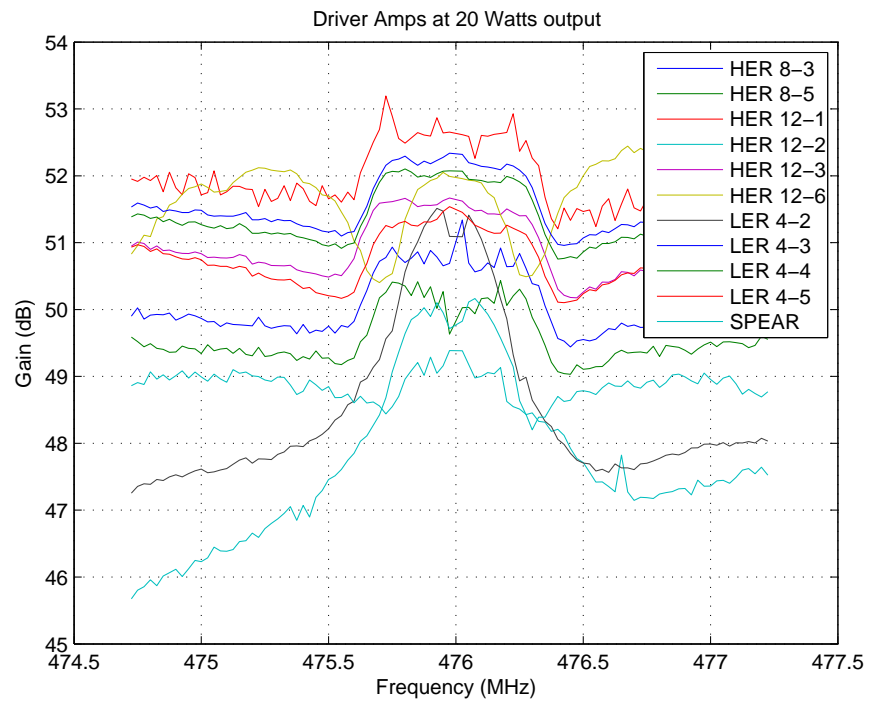

Figure C.5: Small Signal Transfer Function of PEP-II Driver Amplifiers at a largesignal power of 20 Watts.

the response at the excitation sideband frequency, a spectrum analyzer is used to look at the maximum power across the band of interest while the excitation sideband signal is swept. A perfectly linear system would display no power at the image frequencies to the left of the center $476 \mathrm{MHz}$ carrier. These intermodulation plots are reported for a large signal power level of 10 and 30 Watts, shown in Figures C.7 and C.8 respectively. As with the frequency responses, HER 12-2 and LER 4-2 are much worse than most. The unusual structure of HER 4-1, 12-5, and 12-6 is also evident.

From later studies better linear driver amplifiers were identified, ordered and installed. The testing methods and the characteristics of the selected amplifiers are described in [56]. The subsequent improvements in performance are described in Section 5.7. 


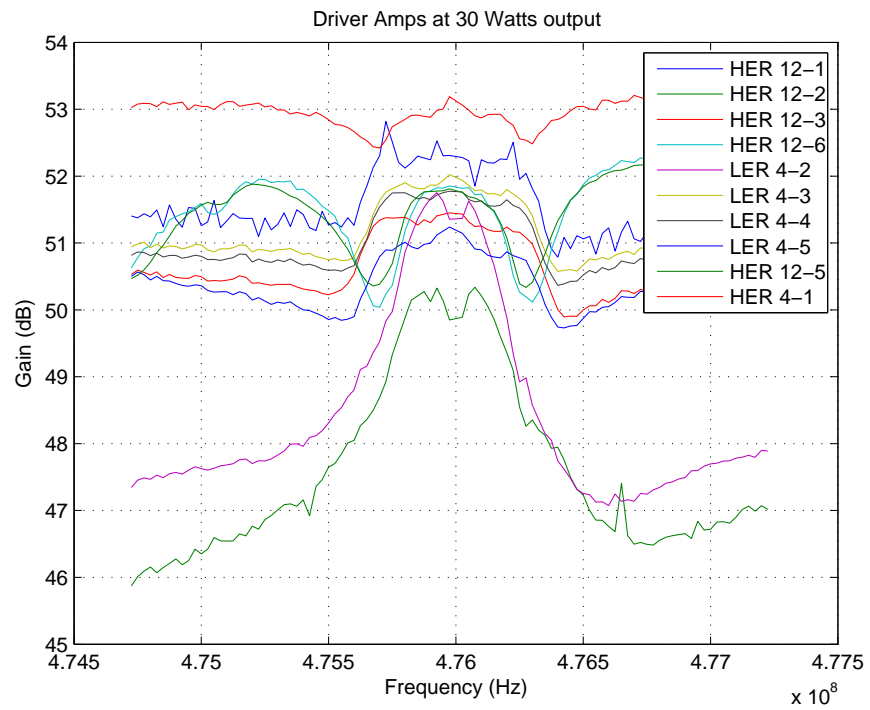

Figure C.6: Small Signal Transfer Function of PEP-II Driver Amplifiers at a largesignal power of 30 Watts.

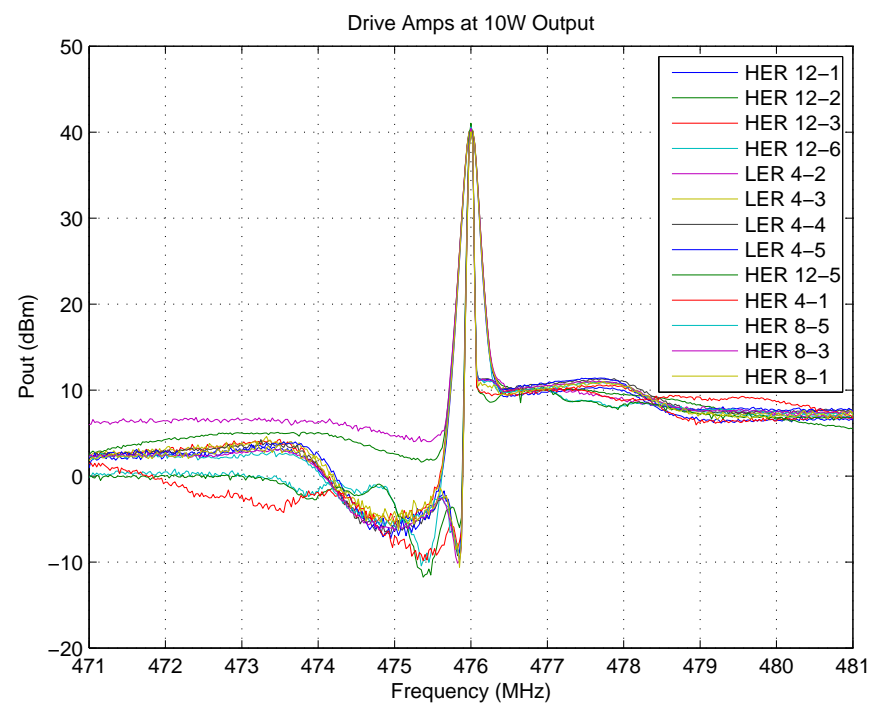

Figure C.7: Intermodulation plots of PEP-II Driver Amplifiers at a large-signal power of 10 Watts. 


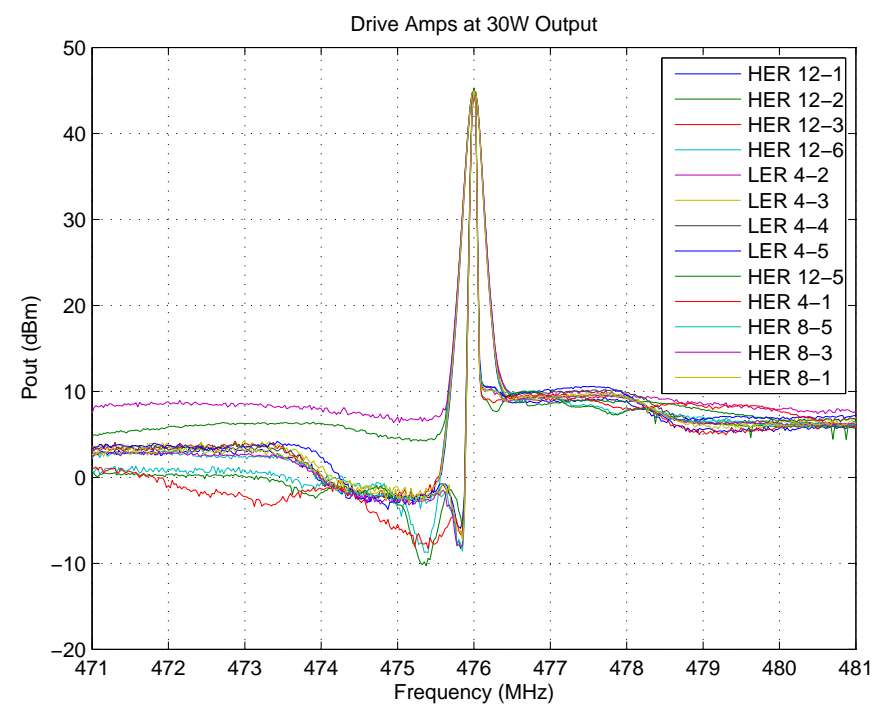

Figure C.8: Intermodulation plots of PEP-II Driver Amplifiers at a large-signal power of 30 Watts. 


\section{Appendix D}

\section{Summary of Accelerator}

\section{Parameters}

The essential accelerator parameters for PEP-II and LHC are summarized in this Chapter.

The Nominal Beam parameters are shown in Table D.1.

\begin{tabular}{|c|c|c|c|}
\hline & LER & HER & LHC \\
\hline Beam energy & $3.1 \mathrm{GeV}$ & $9 \mathrm{GeV}$ & $7 \mathrm{TeV}$ \\
\hline Synchrotron Radiation per turn & $0.65 \mathrm{MeV}$ & $3.55 \mathrm{MeV}$ & $6 \mathrm{keV}$ \\
\hline Max Beam current & $3213 \mathrm{~mA}$ & $2069 \mathrm{~mA}$ & $580 \mathrm{~mA}$ \\
\hline Synchrotron frequency & $4 \mathrm{kHz}$ & $6 \mathrm{kHz}$ & $23 \mathrm{~Hz}$ \\
\hline Bunch Length & \multicolumn{2}{|c|}{$1.13 \mathrm{~cm}$} & $7.5 \mathrm{~cm}$ \\
\hline Longitudinal damping time & \multicolumn{2}{|c|}{$30-50 \mathrm{~s}$} & $13 \mathrm{hours}$ \\
\hline Revolution Frequency & \multicolumn{2}{|c|}{$136.3 \mathrm{kHz}$} & $11.245 \mathrm{kHz}$ \\
\hline
\end{tabular}

Table D.1: PEP-II and LHC Beam parameters

A summary of the RF System parameters is shown in Table D.2. 


\begin{tabular}{|c|c|c|c|}
\hline & LER & HER & LHC \\
\hline 2-cavity RF Stations & 4 & 8 & - \\
\hline 4-cavity RF Stations & - & 3 & - \\
\hline $\begin{array}{c}\text { Normal-conducting } \\
\text { Cavities }\end{array}$ & 8 & 28 & - \\
\hline 1-Cavity Stations & - & - & 8 \\
\hline Superconducting Cavities & - & - & 8 \\
\hline RF frequency & \multicolumn{2}{|c|}{$476 \mathrm{MHz}$} & $400.8 \mathrm{MHz}$ \\
\hline Cavity Voltage & $500-675 \mathrm{kV}$ & $2 \mathrm{MV}$ \\
\hline Cavity loaded Q & \multicolumn{2}{|c|}{6500} & 60,000 \\
\hline Individual Klystron Power & $950-1200 \mathrm{~kW}$ & $330 \mathrm{~kW}$ \\
\hline Klystron Power per beam & $4.8 \mathrm{MW}$ & $13.2 \mathrm{MW}$ & $2.64 \mathrm{MW}$ \\
\hline
\end{tabular}

Table D.2: PEP-II and LHC RF parameters 


\section{Bibliography}

[1] M. Sands, "The Physics of Electron Storage Rings: An Introduction," November 1970. SLAC-PUB-0121.

[2] T. Mastorides, C. Rivetta, J. D. Fox, and D. Van Winkle, "Measurements and Analysis of Longitudinal HOM Driven Coupled Bunch Modes in PEP-II Rings," Contributed to 13th Beam Instrumentation Workshop (BIW08), Lake Tahoe, California, 4-8 May 2008.

[3] S. Prabhakar, New diagnostics and cures for coupled-bunch instabilities. PhD thesis, Stanford University Applied Physics Department, August 2001.

[4] D. Teytelman, Architectures and algorithms for control and diagnostics of coupled-bunch instabilities in circular accelerators. PhD thesis, Stanford University Electrical Engineering Department Department, June 2003.

[5] A. W. Chao, Physics of collective beam instabilities in high-energy accelerators. New York, USA: Wiley (1993) 371 p.

[6] H. Wiedemann, Particle Accelerator Physics; 3rd ed. Berlin: Springer, 2007.

[7] C. Rivetta, T. Mastorides, J. D. Fox, D. Teytelman, and D. Van Winkle, "Modeling and simulation of longitudinal dynamics for Low Energy Ring - High Energy Ring at the Positron-Electron Project," Phys. Rev. ST Accel. Beams, vol. 10, p. 022801, 2007.

[8] P. B. Wilson, "Introduction to Wake Fields and Wake Potentials," AIP Conf. Proc., vol. 184, pp. 525-564, 1989. 
[9] F. Pedersen, "A Novel RF cavity tuning feedback scheme for heavy beam loading," IEEE Trans. Nucl. Sci., vol. 32, pp. 2138-2140, 1985.

[10] S. Prabhakar et al., "Observation and modal analysis of coupled-bunch longitudinal instabilities via a digital feedback control system," Part. Accel., vol. 57, pp. 175-187, 1997.

[11] A. Novokhatski, "HOM Effects in Vacuum System with Short Bunches," Presented at Particle Accelerator Conference (PAC 07), Albuquerque, New Mexico, 25-29 Jun 2007.

[12] R. A. Rimmer, J. Byrd, M. Irwin, and D. A. Goldberg, "Updated impedance estimate of the PEP-II RF cavity," Talk given at 5th European Particle Accelerator Conference (EPAC 96), Sitges, Spain, 10-14 Jun 1996.

[13] F. Pedersen, "Beam Loading Effects in the CERN PS Booster," IEEE Trans. Nucl. Sci., vol. 22, pp. 1906-1909, 1975.

[14] D. Boussard, "Stability considerations," Prepared for 7th LEP Performance Workshop, Chamonix, France, 13-17 Jan 1997.

[15] L. D. Landau, "On the vibrations of the electronic plasma," J. Phys. (USSR), vol. 10, pp. 25-34, 1946.

[16] H. G. Hereward, "Landau Damping," Proceedings, International School Of Particle Accelerators (CERN 77-13), Geneva 1977, 219-230.

[17] A. Mosnier, "Cures of coupled bunch instabilities," Contributed to IEEE Particle Accelerator Conference (PAC 99), New York, 29 Mar - 2 Apr 1999.

[18] D. Boussard, "Cures of instabilities," Prepared for CERN Accelerator School: Course on Advanced Accelerator Physics (CAS), Rhodes, Greece, 20 Sep - 1 Oct 1993.

[19] W. Chou and J. Griffin, "Impedance scaling and impedance control," Talk given at 17th IEEE Particle Accelerator Conference (PAC 97): Accelerator Science, Technology and Applications, Vancouver, Canada, 12-16 May 1997. 
[20] T. Weiland, "Low impedance vacuum chambers," Part. Accel., vol. 51, pp. 53-61, 1995.

[21] R. Rimmer et al., "Higher order mode damping studies on the PEP-II B-factory RF cavity," Prepared for 3rd European Particle Accelerator Conference (EPAC 92), Berlin, Germany, 24-28 Mar 1992.

[22] M. Svandrlik, A. Fabris, and C. Pasotti, "Improved methods of measuring and curing multibunch instabilities in ELETTRA," Prepared for 5th European Particle Accelerator Conference (EPAC 96), Sitges, Spain, 10-14 Jun 1996.

[23] K. Robinson, "Stability of beam in radiofrequency system," Tech. Rep. CEAL1010, CEA, Cambridge, Massachusetts, 1964.

[24] P. Corredoura, L. Sapozhnikov, and R. Tighe, "RF feedback development for the PEP-II B factory," Presented at 4th European Particle Accelerator Conference (EPAC 94), London, England, 27 Jun - 1 Jul 1994.

[25] D. Boussard and G. Lambert, "Reduction of the Apparent Impedance of Wide Band Accelerating Cavities by RF Feedback," IEEE Trans. Nucl. Sci., vol. 30, p. 2239, 1983.

[26] F. Pedersen, "RF cavity feedback," In Proceedings, B factories, Stanford 1992, 192-207. (see HIGH ENERGY PHYSICS INDEX 30 (1993) No. 5432).

[27] R. D. Kohaupt, "Theory of multibunch feedback systems," 1991. DESY-91-071.

[28] D. Boussard, "RF and feedback systems," Prepared for 3rd Workshop on the Tau-Charm Factory, Marbella, Spain, 1-6 Jun 1993.

[29] K. Balewski, "Review of feedback systems," Prepared for 6th European Particle Accelerator Conference (EPAC 98), Stockholm, Sweden, 22-26 Jun 1998.

[30] D. Teytelman, D. Van Winkle, and J. D. Fox, "Operating performance of the low group delay woofer channel in PEP-II," Presented at Particle Accelerator Conference (PAC 05), Knoxville, Tennessee, 16-20 May 2005. 
[31] D. Teytelman, L. Beckman, D. Van Winkle, J. D. Fox, and A. Young, "Development and testing of a low group-delay woofer channel for PEP-II," Presented at the 9th European Particle Accelerator Conference (EPAC 2004), Lucerne, Switzerland, 5-9 Jul 2004.

[32] G. Oxoby et al., "Bunch-by-bunch longitudinal feedback system for PEP-II," Presented at 4th European Particle Accelerator Conference (EPAC 94), London, England, 27 Jun - 1 Jul 1994.

[33] J. D. Fox et al., "Multi-bunch instability diagnostics via digital feedback systems at PEP-II, DAPHNE, ALS and SPEAR," Invited talk at IEEE Particle Accelerator Conference (PAC 99), New York, NY, 29 Mar - 2 Apr 1999.

[34] J. D. Fox et al., "Programmable DSP-based multi-bunch feedback: Operational experience from six installations," Presented at 9th Beam Instrumentation Workshop (BIW 2000), Cambridge, Massachusetts, 8-11 May 2000.

[35] H. Hindi, D. Briggs, J. D. Fox, W. Hosseini, and A. Hutton, "Downsampled bunch by bunch feedback for PEP-II," Presented at B Factories: the State of the Art in Accelerators, Detectors, and Physics, Stanford, CA, 6-10 Apr 1992.

[36] D. Teytelman et al., "Control of Multibunch Longitudinal Instabilities and Beam Diagnostics Using a DSP based Feedback System," 17th IEEE Particle Accelerator Conference (PAC 97): Accelerator Science, Technology and Applications, 12-16 May 1997, Vancouver, British Columbia, Canada.

[37] D. Teytelman et al., "Beam diagnostics based on time-domain bunch-by-bunch data," Presented at 8th Beam Instrumentation Workshop (BIW 98), Stanford, California, 4-7 May 1998.

[38] R. Claus et al., "Software architecture of the longitudinal feedback system for PEP-II, ALS and DAPHNE," Prepared for 16th IEEE Particle Accelerator Conference (PAC 95) and International Conference on High-energy Accelerators (IUPAP), Dallas, Texas, 1-5 May 1995. 
[39] S. Khan and T. Knuth, "BESSY II feedback systems," Prepared for IEEE Particle Accelerator Conference (PAC 99), New York, New York, 29 Mar - 2 Apr 1999.

[40] Y. Kim, J. Y. Huang, M. Kwon, and I. S. Ko, "Status of longitudinal feedback system for the PLS storage ring," Prepared for IEEE Particle Accelerator Conference (PAC 99), New York, New York, 29 Mar - 2 Apr 1999.

[41] J. D. Fox, T. Mastorides, C. Rivetta, D. Van Winkle, and D. Teytelman, "Lessons Learned from Positron-Electron Project Low Level RF and Longitudinal Feedback," Phys. Rev. ST Accel. Beams, vol. 13, p. 052802, May 2010.

[42] P. Corredoura, "Architecture and performance of the PEP-II low-level RF system," Presented at IEEE Particle Accelerator Conference (PAC 99), New York, NY, 29 Mar - 2 Apr 1999.

[43] D. Teytelman, "PEP-II Low-level RF tutorial," August 2003.

[44] F. Voelker and G. Lambertson, "Calculations of RF cavity feedback using a simple analytic model," SLAC-ABC-27.

[45] R. Tighe and P. Corredoura, "RF feedback simulation results for PEP-II," Contributed to 16th IEEE Particle Accelerator Conference (PAC 95) and International Conference on High Energy Accelerators (IUPAP), Dallas, Texas, 1-5 May 1995.

[46] T. Mastorides and C. Rivetta, "Non-Linear Time-Domain Simulations of the RF Station-Beam Dynamics Interaction for the LHC," SLAC-TN-10-025, August 2010.

[47] D. Teytelman, "A non-invasive technique for configuring low level RF feedback loops in PEP-II," Presented at Particle Accelerator Conference (PAC 05), Knoxville, Tennessee, 16-20 May 2005. 
[48] U. Wienands et al., "High-current Effects in the PEP-II Storage Rings," Contributed to 11th European Particle Accelerator Conference (EPAC 08), Magazzini del Cotone, Genoa, Italy, 23-27 Jun 2008.

[49] E. Jongewaard et al., "Operating results for the PEP-II 1.2-MW klystron," Talk given at 5th European Particle Accelerator Conference (EPAC 96), Sitges, Spain, 10-14 Jun 1996.

[50] U. Wienands, Y. Cai, S. D. Ecklund, J. T. Seeman, and M. K. Sullivan, "PEP-II at $1.2 \times 10^{34} / \mathrm{cm}^{2} / \mathrm{s}$ luminosity," Invited talk at Particle Accelerator Conference (PAC 07), Albuquerque, New Mexico, 25-29 Jun 2007.

[51] A. Novokhatski Invited talk at Particle Accelerator Conference (PAC 05), Knoxville, Tennessee, 16-20 May 2005.

[52] "PEP-II: An Asymmetric B Factory. Conceptual Design Report. June 1993," SLAC-R-418.

[53] J. D. Fox et al., "Klystron linearizer for use with 1.2-MW 476-MHz klystrons in PEP-II RF systems," Presented at Particle Accelerator Conference (PAC 05) 16-20 May 2005, Knoxville, Tennessee.

[54] D. Van Winkle et al., "Amplitude linearizers for PEP-II 1.2-MW klystrons and LLRF systems," Prepared for European Particle Accelerator Conference (EPAC 06), Edinburgh, Scotland, 26-30 Jun 2006.

[55] T. Mastorides, C. Rivetta, J. D. Fox, D. Teytelman, and D. Van Winkle, "Analysis of the longitudinal low-order mode beam dynamics in PEP-II rings at high current beams," Presented at Particle Accelerator Conference (PAC 07), Albuquerque, New Mexico, 25-29 Jun 2007.

[56] J. D. Fox, T. Mastorides, C. H. Rivetta, and D. Van Winkle, "Selecting RF amplifiers for impedance controlled LLRF systems: Nonlinear effects and system implications," Presented at Particle Accelerator Conference (PAC 07), Albuquerque, New Mexico, 25-29 Jun 2007. 
[57] D. Van Winkle et al., "Feedback Configuration Tools for LHC Low Level RF System," Presented at Particle Accelerator Conference (PAC 09), Vancouver, BC, Canada, 4-8 May 2009.

[58] D. Van Winkle et al., "Commissioning of the LHC Low Level RF System Remote Configuration Tools," Presented at International Particle Accelerator Conference (IPAC 10), Kyoto, Japan, 23-28 May 2010.

[59] P. Baudrenghien et al., "The LHC Low Level RF," Proceedings of European Particle Accelerator Conference (EPAC 06), Edinburgh, Scotland, 26-30 Jun 2006.

[60] J. Holma, "The Model and Simulations of the LHC $400 \mathrm{MHz}$ Cavity Controller," CERN-AB-2007-012, CERN, Feb. 2007.

[61] J. Tuckmantel, "Simulation of LHC Bunches under Influence of 50-Hz multiple Lines on the Cavity Field," LHC Project Note-404, June 2007.

[62] K. Astrom, Introduction to Stochastic Control Theory. Dover Publications, 1970.

[63] J. W. H. Stark, Probability, Random Processes, and Estimation Probability, Random Processes, and Estimation Theory for Engineers. Prentive Hall, 1994.

[64] T. Leiber, F. Marchesoni, and H. Risken, "Colored noise and bistable FokkerPlanck equations," Phys. Rev. Lett., vol. 59, pp. 1381-1384, Sep 1987.

[65] A. Burov, V. Lebedev, and Y. Alexahin, "Diffusion of Nonlinear Oscillators and Its Application to RF Noise," Private Communication.

[66] J. Tuckmantel, "Synchrotron Radiation Damping in LHC and Longitudinal Bunch Shape," Tech. Rep. LHC-Project-Report-819. CERN-LHC-ProjectReport-819, CERN, Geneva, Jun 2005.

[67] T. Mastorides, C. Rivetta, J. D. Fox, D. Van Winkle, and D. Teytelman, "Analysis of Longitudinal Beam Dynamics Behavior and RF System Operative Limits at High Beam Currents in Storage Rings," Phys. Rev. ST Accel. Beams, vol. 11, p. 062802, 2008. 
[68] P. Baudrenghien, "The Tuning Algorithm of the LHC 400 MHz Superconducting Cavities," Tech. Rep. AB-Note-2007-011. CERN-AB-Note-2007-011, CERN, Feb 2007. revised version submitted on 2007-02-05 12:19:31.

[69] D. Boussard, "RF power estimates for a hadron collider," Tech. Rep. CERN-SPSARF-DB-gw-Note-84-9. LHC-NOTE-10. CERN-LHC-Note-10, CERN, Geneva, 1984.

[70] D. Boussard, "RF power requirements for a high intensity proton collider," p. 7 p, May 1991.

[71] V. Rossi, "Digital Signal Processing for 1-Turn Delay Feedback Systems of the CERN Accelerator Chain," Tech. Rep. CERN-BE-2009-009, CERN, Geneva, Jan 2009.

[72] D. Boussard, D. Brandt, and L. Vos, "Is a longitudinal feedback system required for LHC?," Tech. Rep. LHC-PROJECT-NOTE-205, CERN, Geneva, Oct 1999.

[73] E. Shaposhnikova, "Longitudinal beam parameters during acceleration in the LHC," Tech. Rep. LHC-PROJECT-NOTE-242, CERN, Geneva, Dec 2000.

[74] T. Suzuki, Y. H. Chin, and K. Satoh Part. Accelerators, vol. 179, no. 13, 1983.

[75] M. H. Blewett, (Ed. ), "Theoretical aspects of the behavior of beams in accelerators and storage rings: International School of Particle Accelerators of the 'Ettore Majorana' Center for Scientific Culture, Erice, Italy, 10-22 Nov. 1976: Proceedings," p. 139. CERN-77-13.

[76] F. Ruggiero, "Single-beam collective effects in the LHC," Part. Accel., vol. 50, pp. 83-104. 21 p, Feb 1995.

[77] F. J. Sacherer, "A Longitudinal stability criterion for bunched beams," IEEE Trans. Nucl. Sci., vol. 20, pp. 825-829, 1973.

[78] T. Mastorides, C. Rivetta, J. D. Fox, D. Van Winkle, and D. Teytelman, "RF Noise Effects on Large Hadron Collider Beam Diffusion," Submitted for Publication to Phys. Rev. ST Accel. Beams. 
[79] T. Mastorides, C. Rivetta, J. D. Fox, D. Van Winkle, and D. Teytelman, "LHC Beam Diffusion Dependence on RF Noise: Models and Measurements," Presented at International Particle Accelerator Conference (IPAC 10), Kyoto, Japan, 23-28 May 2010.

[80] T. Mastorides, C. Rivetta, J. D. Fox, and D. Van Winkle, "Application of NonLinear Time-Domain RF Simulations to Longitudinal Emittance Studies for the LHC," Contributed to Particle Accelerator Conference (PAC 09), Vancouver, BC, Canada, 4-8 May 2009.

[81] G. Papotti, "Beam Quality Monitor (BQM)." Private Communication.

[82] R. Assmann et al., "Beam Parameters and Machine Performance to be Reached in 2010," Proceedings of the 2010 Evian workshop on LHC commissioning, Evian, France, 19-20th January 2010.

[83] "LHC Design Report," Volume 1, Chapter 2, CERN-2004-003, 4 June 2004.

[84] T. Mastorides et al., "Modeling and Simulation of the Longitudinal Beam Dynamics - RF Station Interaction in the LHC Rings," Contributed to 11th European Particle Accelerator Conference (EPAC 08), Magazzini del Cotone, Genoa, Italy, 23-27 Jun 2008.

[85] D. Teytelman, J. D. Fox, and L. Doolittle, "Digital LLRF." Private Communication.

[86] L. Doolittle, "Low-Level RF Control System Design and Architecture," Presented at Asian Particle Accelerator Conference 2007, Indore, India, January 29-February 2007.

[87] D. Teytelman and J. D. Fox, "Set-up of PEP-II longitudinal feedback systems for even/odd bunch spacings," AIP Conf. Proc., vol. 648, pp. 474-482, 2003.

[88] H. Hindi et al., "Analysis of DSP-based longitudinal feedback system: Trials at SPEAR and ALS," Presented at 1993 Particle Accelerator Conference (PAC 93), Washington, DC, 17-20 May 1993. 
[89] C. Rivetta, T. Mastorides, J. D. Fox, and D. Van Winkle, "PEP-X: RF Issues and Initial Estimates," SLAC-TN-08-003, December 2008.

[90] K. Bane et al., "Lattice and Collective Effects for PEP-X," April 2008. SLACPUB-13225. 